\title{
Bringing life to leadership: the emergence of principal leadership practice for educational success in low socio-economic schools
}

\author{
by
}

Angela Blanche Anna-Lise Millar

\author{
A thesis submitted to \\ Victoria University of Wellington \\ in fulfilment of the requirements for the degree \\ of \\ Doctor of Philosophy
}

Victoria University of Wellington

2019 


\begin{abstract}
The persistent educational achievement gap and historically haphazard nature of progress for students in impoverished contexts in New Zealand primary schools is a deep-seated problem needing urgent attention. This multifaceted and interconnected problem has no distinct origin and no clear solution and is continuing to harm individual and collective futures. This thesis explores principal leadership practice and how it intersects with the low socio-economic context, to improve outcomes for vulnerable students.
\end{abstract}

Using a case study approach, two principals and their schools are holistically described using a theoretical framework grounded in complexity theory. Both the approach and theory uniquely explores principal practice as an individual and collective phenomenon, as a living, evolving network of self-organisation where both parts and wholes are significant, and as practice grounded in context.

The theory also offers a unique way to arrange and interpret findings. The study findings identified local notions of educational success as a way of understanding the trajectory of the ecosystem whole. The broad principal leadership patterns of practice included: building the network; integrating multiple perspectives; facilitating cohesion; and confronting and addressing injustices. These patterns evidenced a tight coupling between theory and practice. In the spirit of complexity theory, principal leadership practice is additionally explained as a sum greater than its parts. The capability of the network is described through concepts of stability and flow and shows, that no matter how competent the principal leader and their network, the contribution to education reform grapples to be transformational.

Recommendations are made for principal leaders, professional development providers, as well as the Ministry of Education and policymakers that offer hope and contribute to disrupting the current trajectory, so transformational change is more likely. Areas of instability and important leverage points, include professional learning and development, community-school connections and resourcing. 


\section{Acknowledgments}

I call it cruel and maybe the root of all cruelty

To know what occurs but not recognize the facet.

And so, I appeal to a voice, to something shadowy,

A remote important region in all who talk:

Though we could fool each other, we should consider-

Lest the parade of our mutual life get lost in the dark.

For it is important that awake people be awake,

Or a breaking line may discourage them back to sleep;

The signals we give- yes or no, or maybe-

Should be clear; the darkness around us is deep.

(Stafford, 1998)

This thesis was made possible by my research participants who allowed me to be a part of their day-to-day lives. The principals especially, were wonderfully generous with their time and trusting of the process. Faafetai and whakawhetai koe.

I acknowledge my supervisors Kate Thornton, Liz Jones and Elizabeth Eppel who were never short of kindness, critique and wisdom. My thanks to them as individuals but especially to the dynamic supervisory team we became together.

Colleagues Fueapepe Rimoni, Jan Stewart, Kathryn Argyle, Linda Hogg, Linda Bonne, Lesley Murithy, Mabel Hamon, my many Bootcamp buddies, Rose Hipkins who offered especially strong counsel and the late Don Brown and Lottie Thomson who believed in me from the start of my academic endeavours - you are all great reminders that each of us carries the capacity to change the world for the better.

In celebration of the lives of family, friends and supporters who passed away while this thesis was in process: Angela Bond, Aunty Bev Walker, Aunty Rosa Kelly, Brigid Conoglan, Jim Douglas, Melissa Sotomayor, Uncle Alois Hauptmann, Uncle Fred Baker, Uncle Ru Whaanga, and Tiffany Williams. Siempre creíste en mí. Ustedes son ángeles arriba y alrededor. Hago esto por todos ustedes. I honour your memories here. 
Finally, this thesis is dedicated to my family. In particular, to my grandmothers Mary Lamacchia and Eileen Millar, and my mother Anna Millar. These three woman taught me what it means to be a strong, resilient female in the face of material hardship and social injustice. To my father Kenneth William Millar, who always wanted a doctor in the family, my brother John William Millar who never got the chance to see out his potential, and to my nieces and nephews who are changing the world for the better - I am so proud to be your aunt. Finally, to my husband Nick Swan, you were there at every obstacle and every celebration and your care, love and enduring belief in me, will stay with me well beyond these pages. 


\section{Table of Contents}
Abstract.
Acknowledgments ii
Table of Contents ............................................................................................................ iv

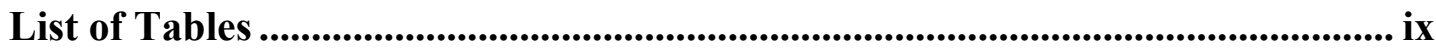
List of Figures....................................................................................................................... $x$

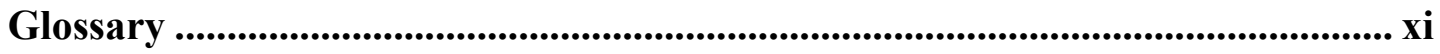

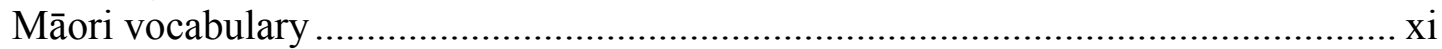

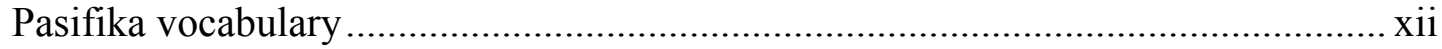

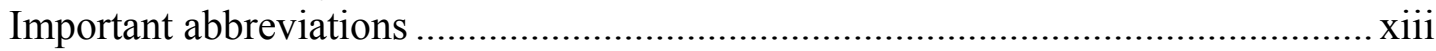

CHAPTER 1 The research focus, motivation and outline ................................. 1

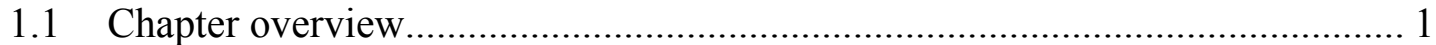

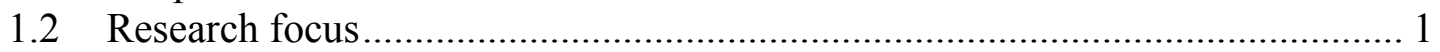

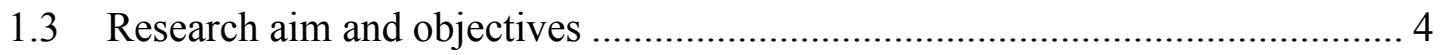

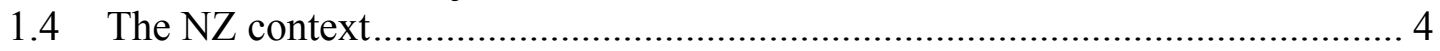

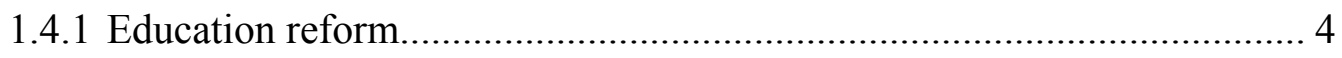

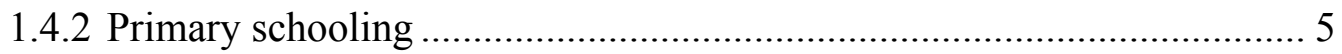

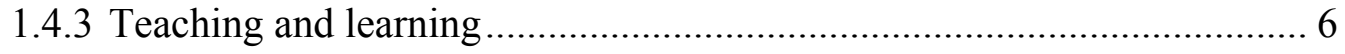

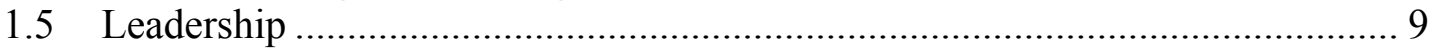

1.5.1 Professional learning and development initiatives for teachers............. 11

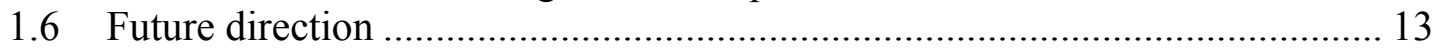

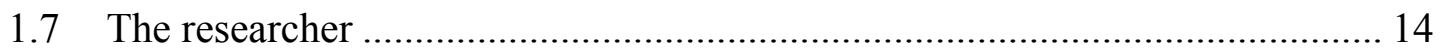

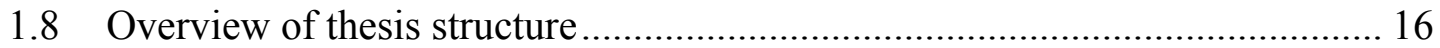

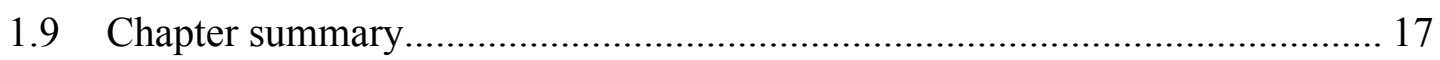

CHAPTER 2 The literature review .................................................................. 18

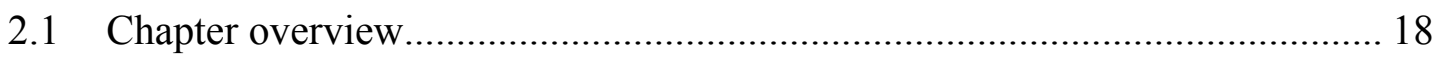

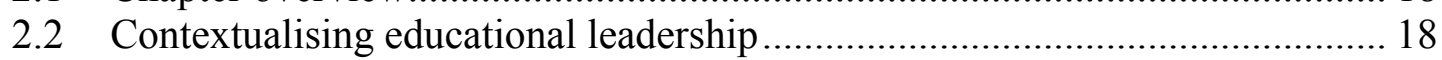

2.3 Themes of educational leadership practice................................................. 19

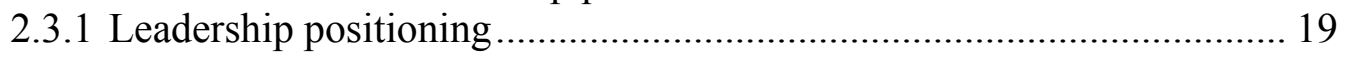

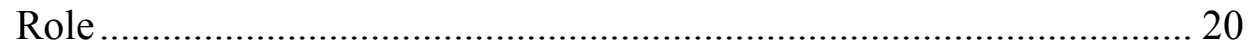

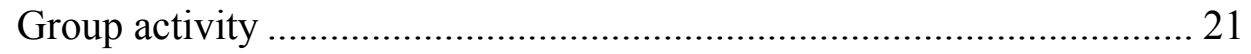

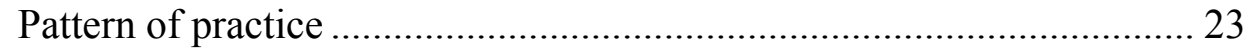

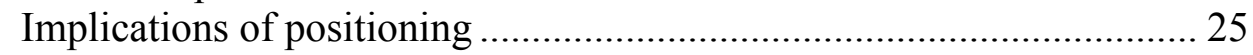

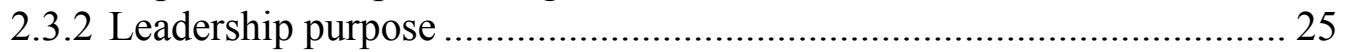

Personal beliefs and values ............................................................... 26

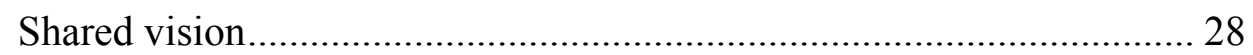

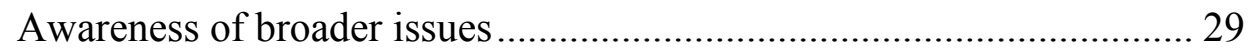

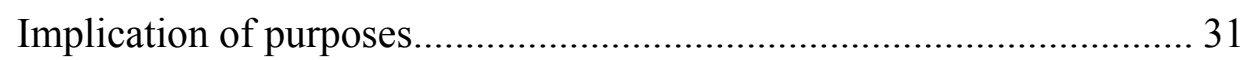

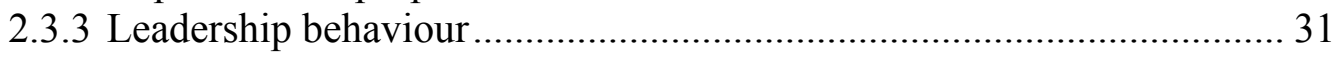

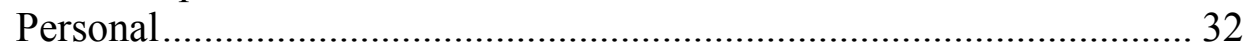

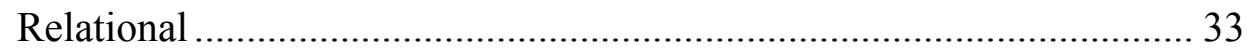

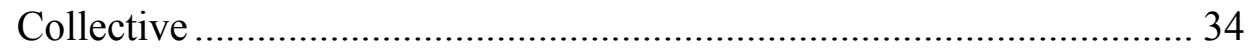

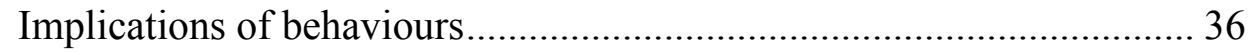

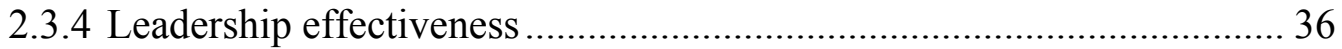




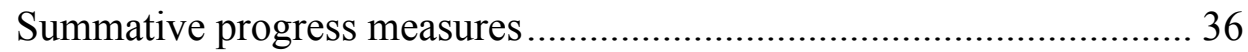

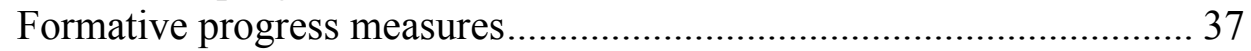

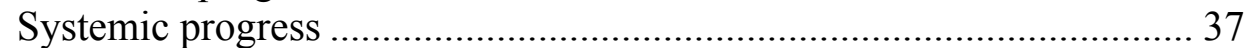

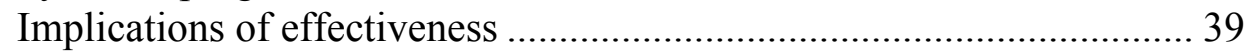

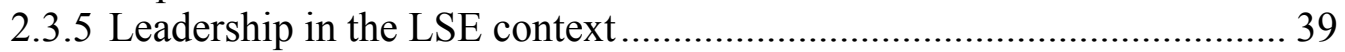

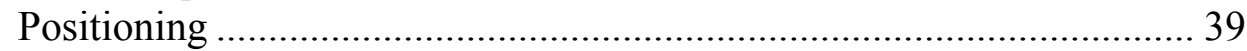

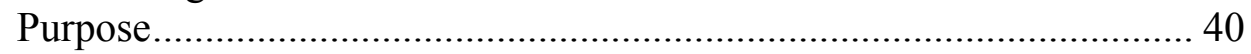

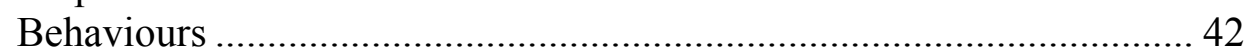

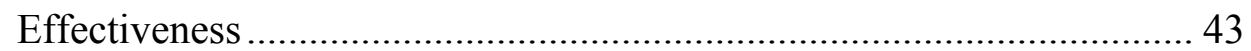

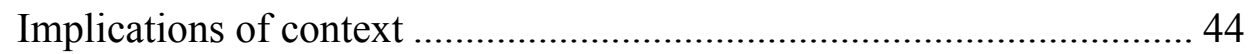

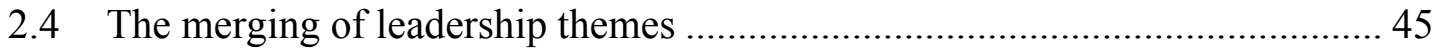

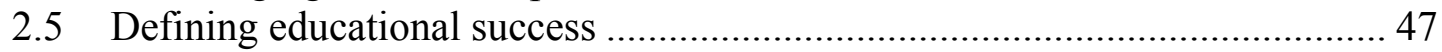

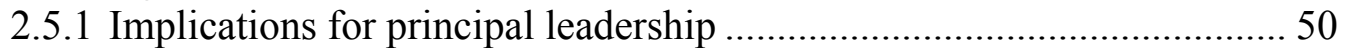

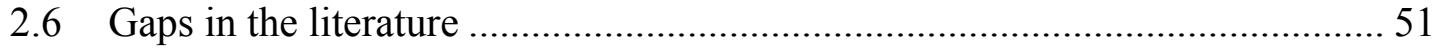

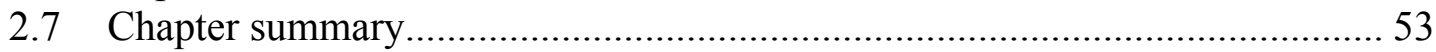

CHAPTER 3 The theoretical framework .....................................................54

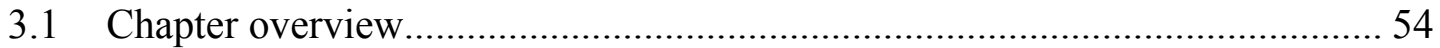

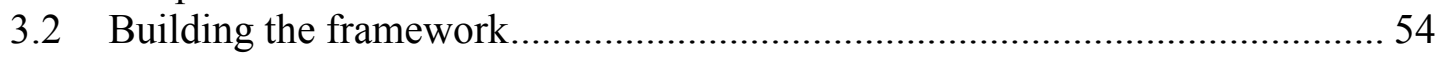

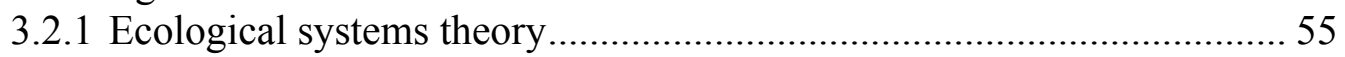

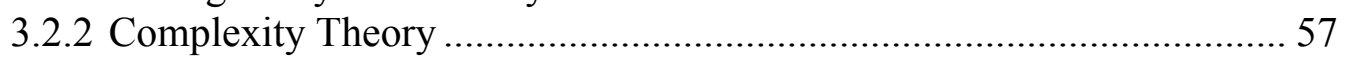

Complexity theory and ecology ........................................................ 59

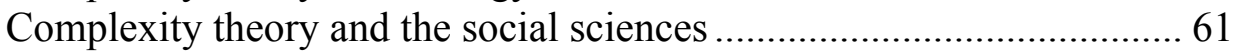

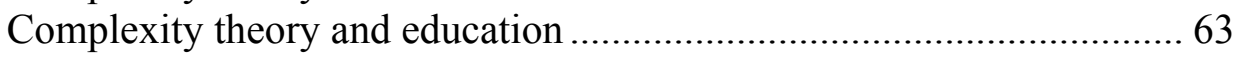

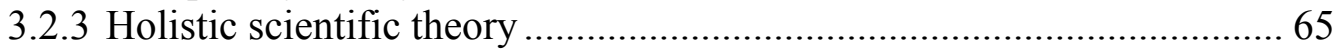

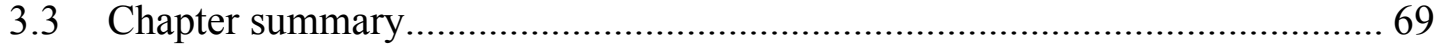

CHAPTER 4 The Methodology

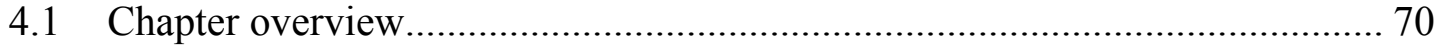

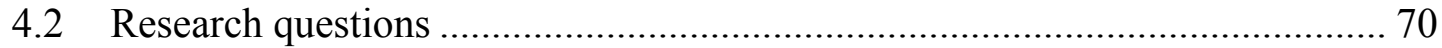

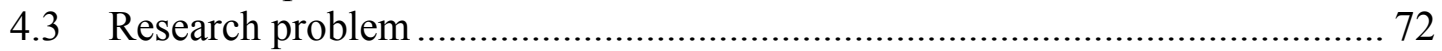

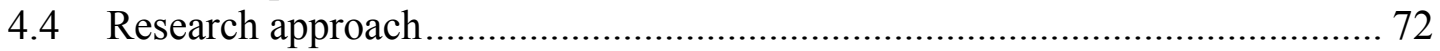

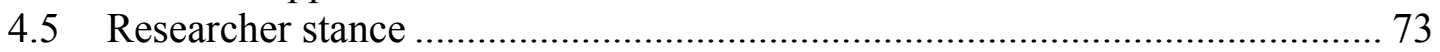

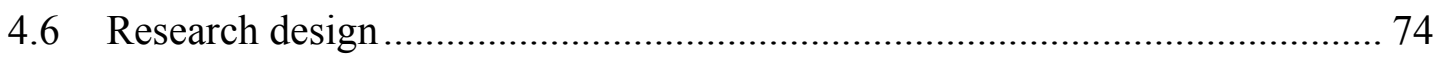

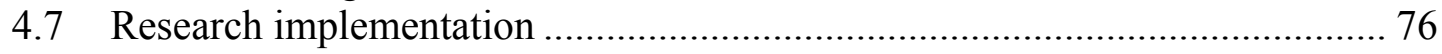

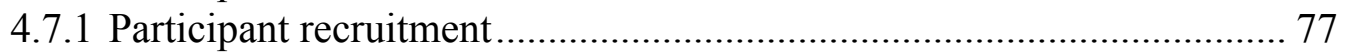

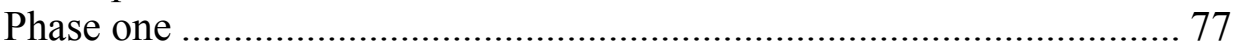

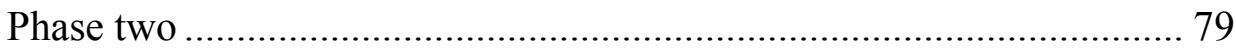

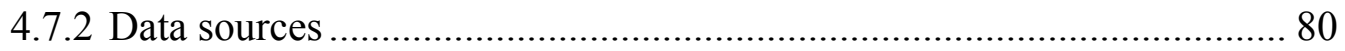

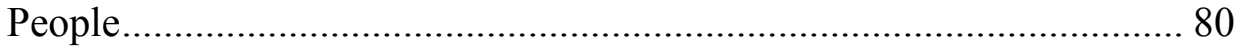

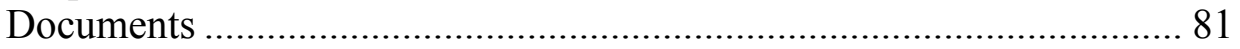

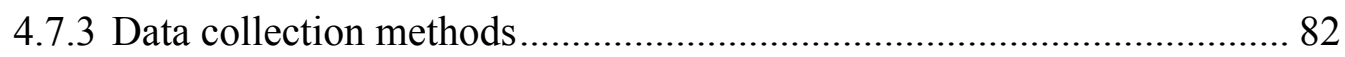

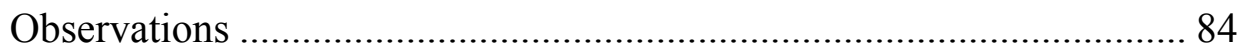

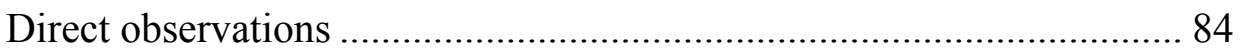

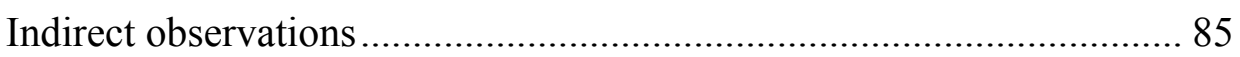

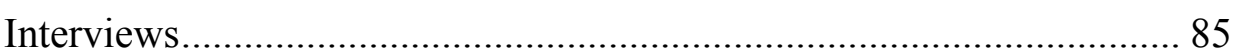

In-depth semi-structured principal interview ...................................... 85

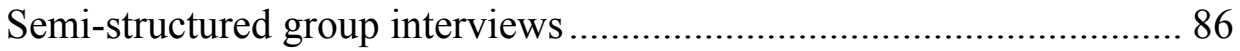

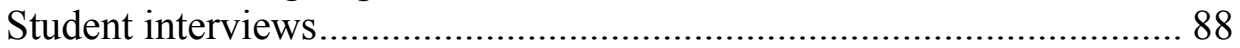

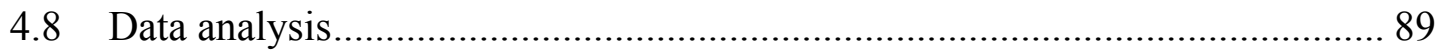

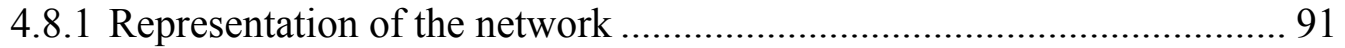

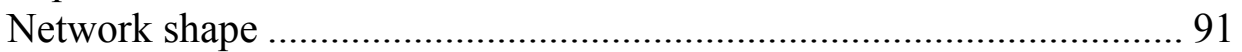

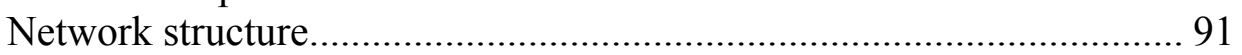




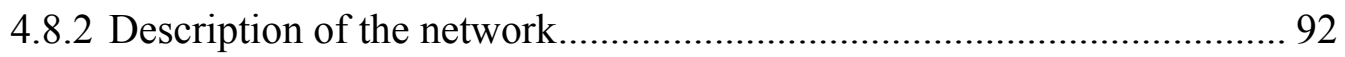

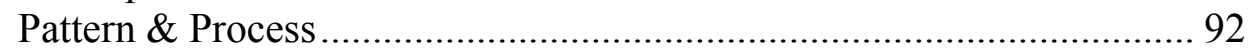

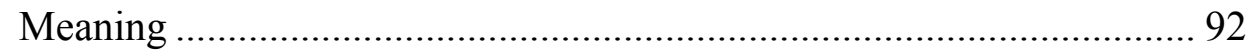

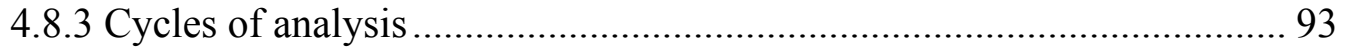

4.8.4 Data triangulation and cross-case analysis .............................................. 94

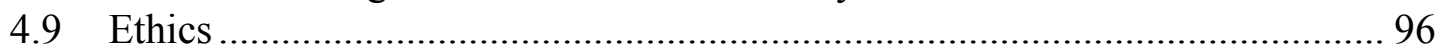

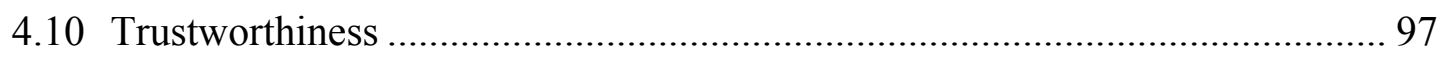

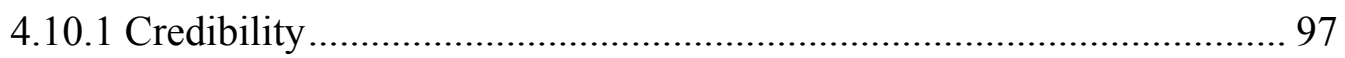

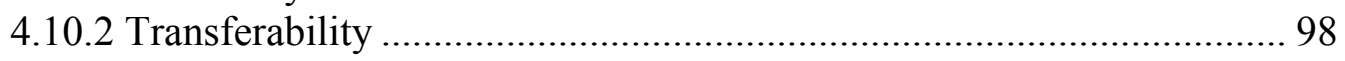

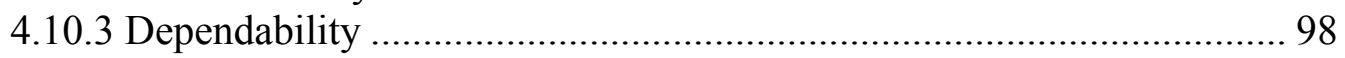

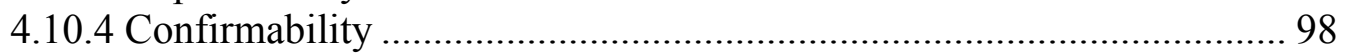

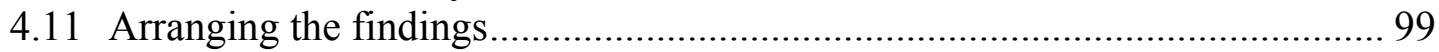

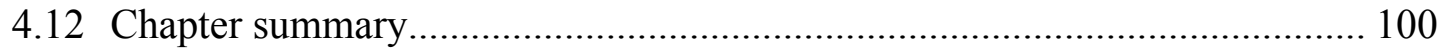

CHAPTER 5 Principal leadership practice at Acacia School ........................... 102

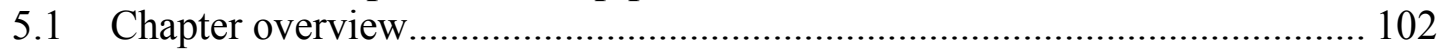

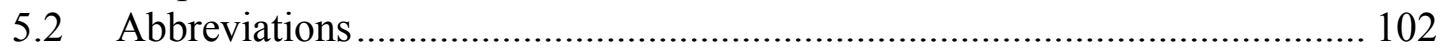

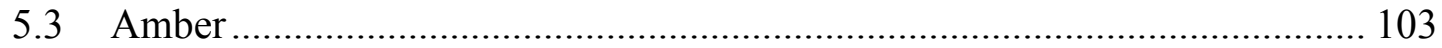

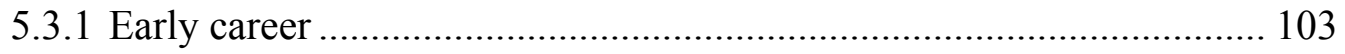

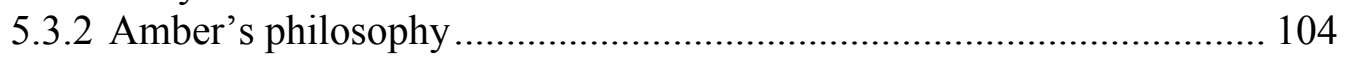

Strong relationships ................................................................... 104

Diversity and belonging ................................................................. 104

Deep thinking and solutions-focused................................................ 105

5.4 Amber as principal of Acacia School .......................................................... 105

5.5 Educational success in the Acacia community............................................ 107

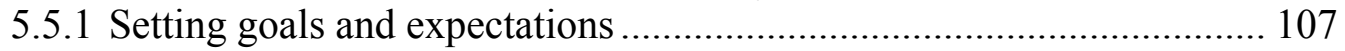

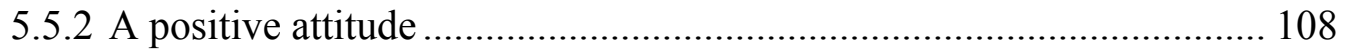

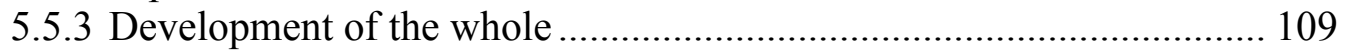

5.6 Representing Amber's principal leadership network ................................... 109

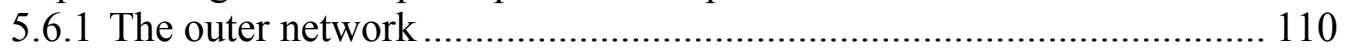

5.6.2 The inner network ........................................................................ 111

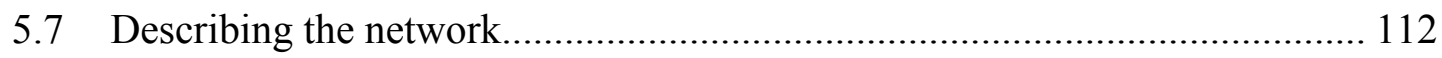

5.7.1 Network One: Acacia School......................................................... 112

Nested Network 1 - Leadership team .............................................. 112

Nested Network 2 - Teaching staff .................................................. 116

Nested Network 3 - Support staff....................................................... 120

Nested Network 4 - Students.......................................................... 123

5.7.2 Network Two: Acacia neighbourhood ............................................. 125

Nested Network 5 - Governance ........................................................ 125

Nested Network 6 - Aiga/whānau ....................................................... 130

Nested Network 7 - Education support workers ............................... 134

5.7.3 Network Three: Wider Acacia community ...................................... 137

Nested Network 8 - Private businesses and organisations ................. 137

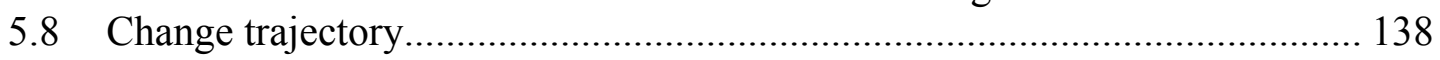

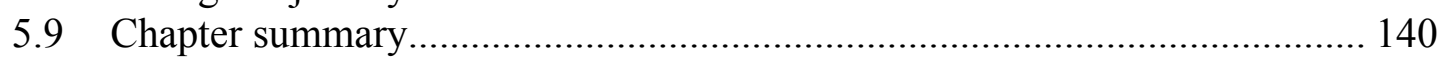

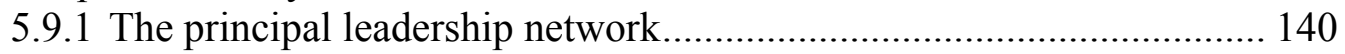

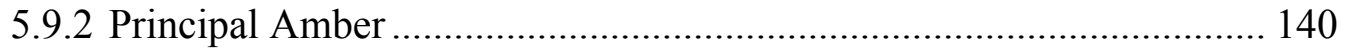

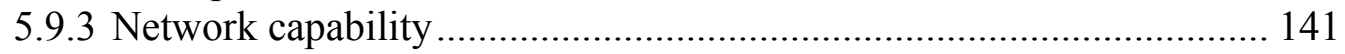

CHAPTER 6 Principal leadership practice at Cedar School ........................... 142

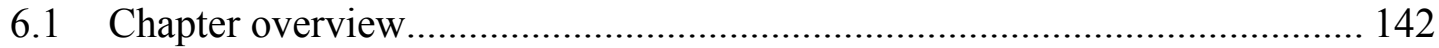

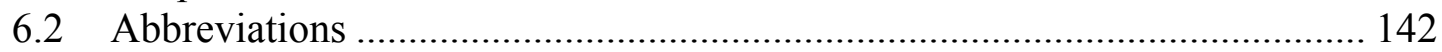

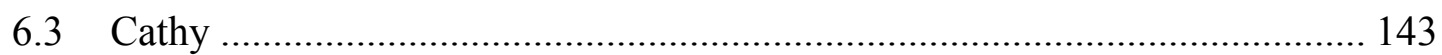




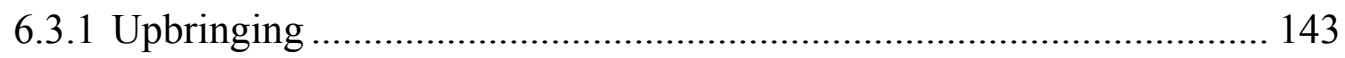

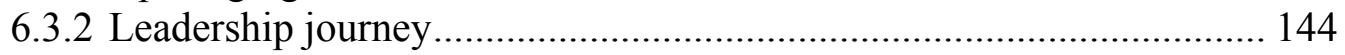

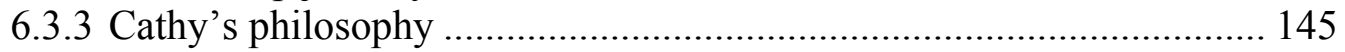

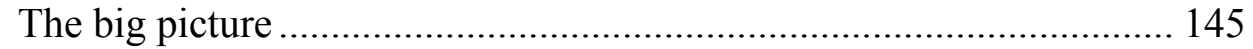

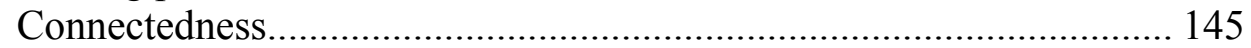

Learner disposition................................................................... 146

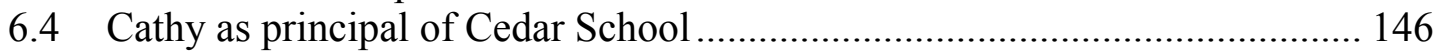

6.5 Educational success in the Cedar community ............................................. 148

6.5.1 Academic improvement ............................................................... 148

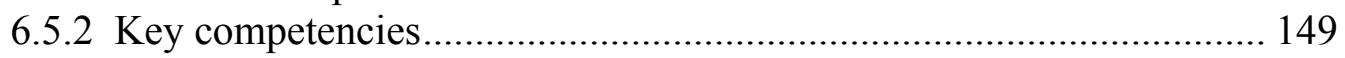

6.5.3 Development of the whole ........................................................... 150

6.6 Representing Cathy's principal leadership network.................................. 151

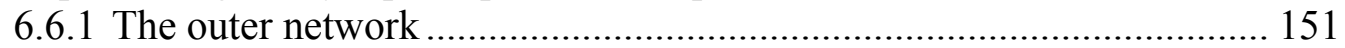

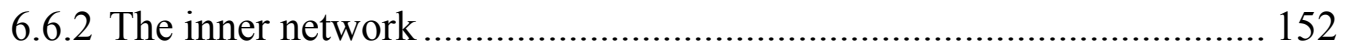

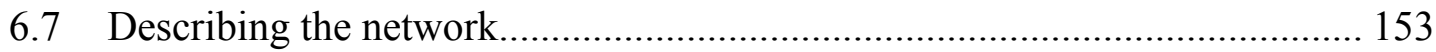

6.7.1 Network One: Cedar School .............................................................. 153

Nested Network 1 - Leadership team................................................. 154

Nested Network 2 - Teaching staff ............................................... 156

Nested Network 3 - School support staff ........................................ 161

Nested Network 4 - Students........................................................... 163

6.7.2 Network Two: Cedar Neighbourhood.................................................. 166

Nested Network 5 - Governance .................................................... 166

Nested Network 6 - Aiga/whānau .................................................. 170

Nested Network 7 - Education support workers ................................. 174

Nested Network 8 - Health and welfare services .............................. 176

6.7.3 Network Three: Wider Cedar Community........................................... 178

Nested Network 9 - Private businesses and organisations .................. 178

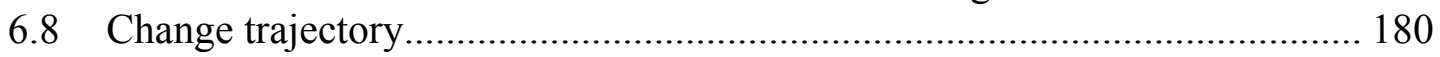

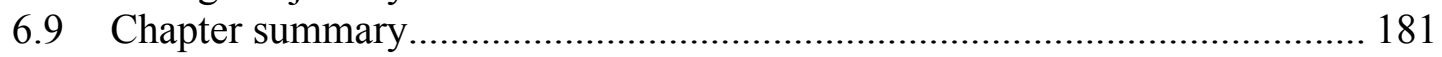

6.9.1 The principal leadership network............................................... 181

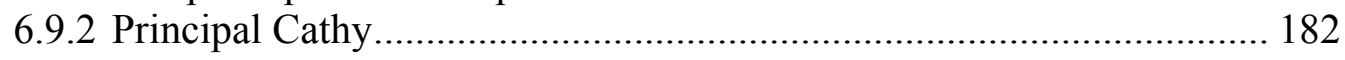

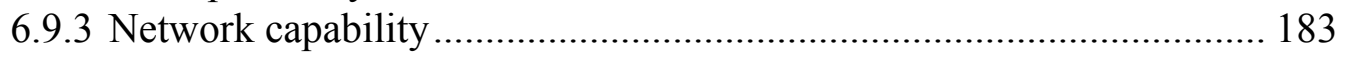

CHAPTER 7 Exploring principal leadership practice........................................ 184

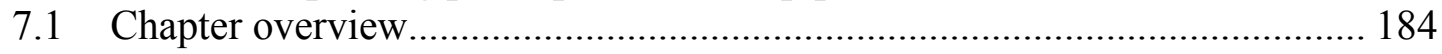

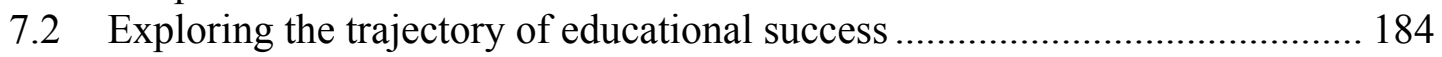

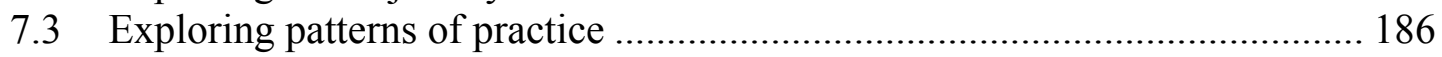

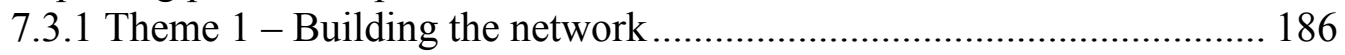

7.3.2 Theme 2 - Integrating multiple perspectives ....................................... 191

7.3.3 Theme 3 - Facilitating cohesion......................................................... 193

7.3.4 Theme 4 - Confronting and addressing injustices ............................. 197

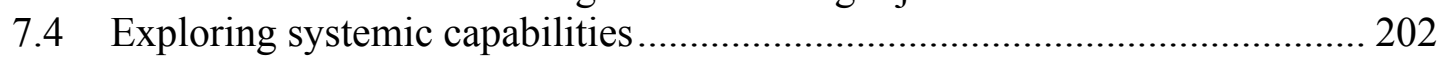

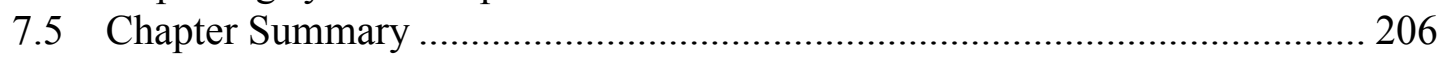

CHAPTER 8 Looking back on the journey........................................................ 209

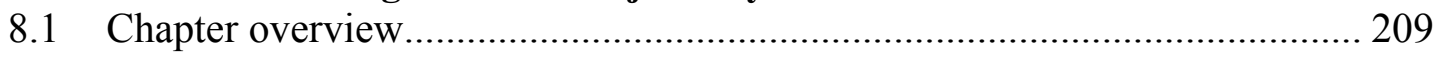

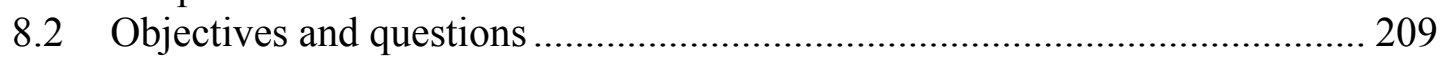

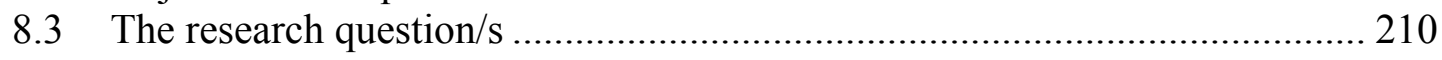

8.4 Answering individual research questions .................................................... 211

8.4.1 What leadership practice patterns are in evidence in the principal leadership network?

8.4.2 What beliefs and ideas underpin a principal's engagement in their context? 
8.4.3 In what ways are principal leadership practice patterns responsive to and influenced by their context?......................................................... 212

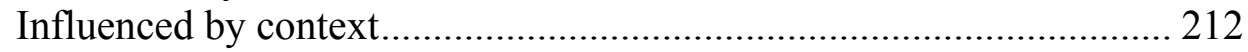

Influence on context.............................................................................. 212

8.4.4 What do the stakeholders in the LSE context perceive as educational success?

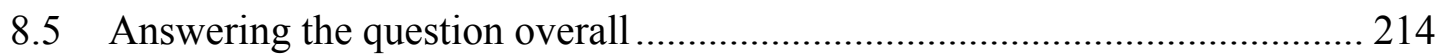

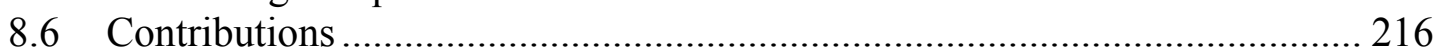

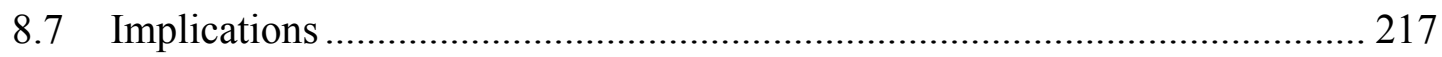

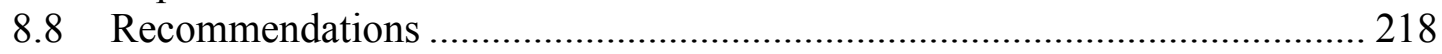

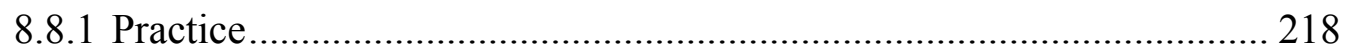

8.8.2 Professional Learning and Development .......................................... 219

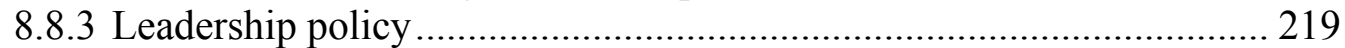

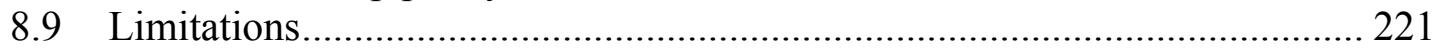

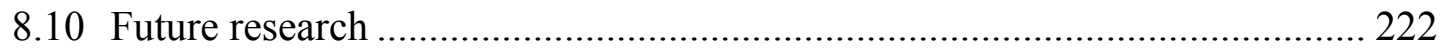

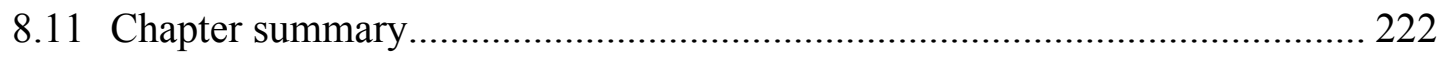

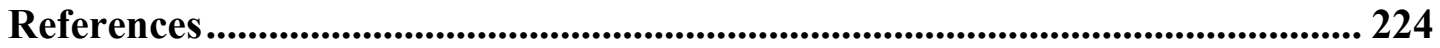

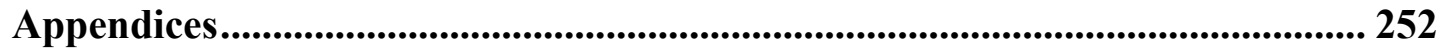

Appendix A: A chronology of relevant NZ developments of educational

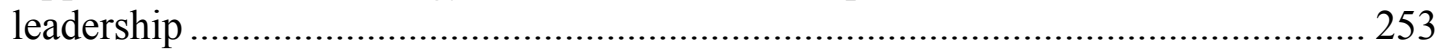

Appendix B: The scope of literature used in the review....................................... 256

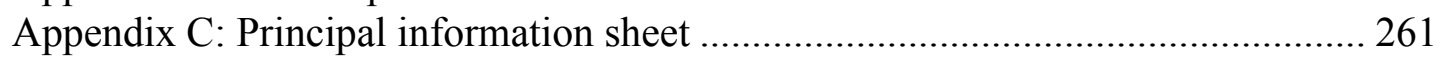

Appendix D: Research schedule - an example.................................................. 265

Appendix E: Researcher field notes - example................................................... 266

Appendix F: Principal semi-structured interview schedule ................................ 267

Appendix G: Various participant semi-structured interview schedule ................... 268

Appendix H: Coded transcript - an example .................................................... 269

Appendix I: Tabular display of coded headings - an example ............................. 270

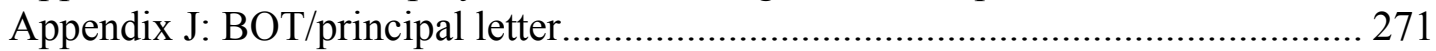

Appendix K: BOT/principal consent forms ........................................................ 272

Appendix L: Information sheets - staff, community and students ........................ 276

Appendix M: Consent forms - staff, community and students ............................ 285

Appendix N: Formal observations- information and consent forms ...................... 291 


\section{List of Tables}

Table 1: Curriculum levels relevant to students in primary schooling in NZ, adapted from the New Zealand Curriclum....................................... 7

Table 2: Linking the literature review to the research questions............................. 53

Table 3: Key case study elements adapted from Gall et al., (2007) ........................ 75

Table 4: Overview of the schools chosen for this case study.....................79

Table 5: The transferal of systems to networks .................................................. 81

Table 6: The timeline of the reseach process in Acacia and Cedar schools ............ 83

Table 7: Staff and community interviews that emerged across the case study ....... 88

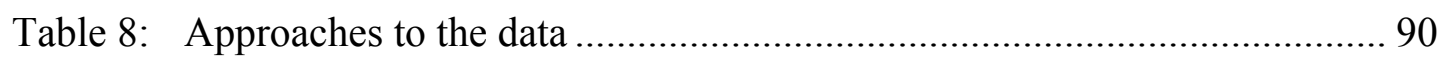

Table 9: The data collection recording codes used throughout this thesis .............. 95

Table 10: Acacia School abbreviations of data sources assigned .......................... 102

Table 11: The feedback loops occuring in Amber's principal leadership

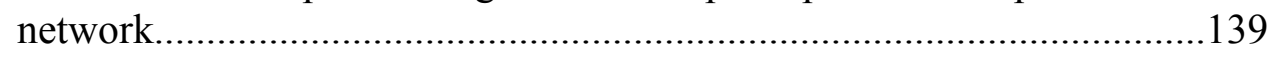

Table 12: Cedar School abbreviations of data sources assigned ........................... 142

Table 13: The feedback loops occuring in Cathy's principal leadership network ...180 


\section{List of Figures}

Figure 1: A continuum of themes and patterns in practice .................................... 45

Figure 2: The building of the theoretical framework................................................ 55

Figure 3: Adaptation of Bronfenbrenner's ecological systems framework model with the principal leader at the centre ................................................... 57

Figure 4: How Amber described her work as principal leader at Acacia School....107

Figure 5: The networks and NN evident in Amber's social relationships

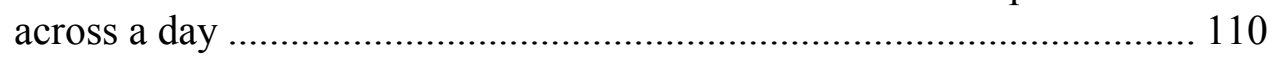

Figure 6: The connections from within Amber's principal leadership network..... 111

Figure 7: How Cathy described her work as principal leader at Cedar School ...... 147

Figure 8: The networks and NN evident in Cathy's social relationships across a day

Figure 9: The NN connections from within Cathy's principal leadership

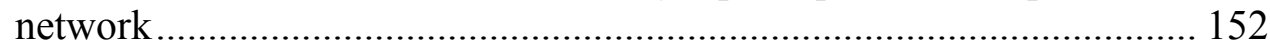

Figure 10:Exploring the principal leadership patterns of practice.......................... 186 


\section{Glossary}

\section{Māori vocabulary}

\begin{tabular}{|c|c|}
\hline Ako & A learning relationship \\
\hline Aotearoa & New Zealand \\
\hline Aroha & Love \\
\hline Awhina & Support \\
\hline Hauora & Health and wellbeing \\
\hline Ka Hikitia & Step up \\
\hline Kapa haka & Group who perform Māori song and dance \\
\hline Kaupapa & History and background \\
\hline Kia Whakaako/Kia ako & Teaching and learning \\
\hline Manaaki/manaakitanga & Care \\
\hline Mana wairua & Spiritual journey \\
\hline Māori & Indigenous Polynesian people of New Zealand \\
\hline Pākehā & New Zealand European \\
\hline Pono & 'to chance upon' \\
\hline Tangi & Funeral \\
\hline Tätaiako & Cultural Competencies for Teachers of Mäori Learners \\
\hline Te reo & Māori language \\
\hline Tikanga & $\begin{array}{l}\text { A wide variety of meanings including culture, customs } \\
\text { and ethics }\end{array}$ \\
\hline Treaty of Waitangi & $\begin{array}{l}\text { Document signed by representatives of the British } \\
\text { Crown and various Māori chiefs to establish a } \\
\text { relationship between the two regarding land and other } \\
\text { properties. }\end{array}$ \\
\hline Whānau & Family \\
\hline
\end{tabular}


Pasifika vocabulary

Aiga

Pasifika

Puletasi
Family

People who identify with the Pacific Islands because of ancestry or heritage

A traditional item of clothing worn by woman in the Pacific 


\section{Important abbreviations}

$\begin{array}{ll}\text { BES } & \text { Best Evidence Synthesis } \\ \text { CAS } & \text { Complex Adaptive System } \\ \text { ELL } & \text { English Language Learners } \\ \text { ESL } & \text { English as a Second Language } \\ \text { ICT } & \text { Information and Communication Technology } \\ \text { LSE } & \text { Low Socio-economic Status } \\ \text { MOE } & \text { Ministry of Education (New Zealand) } \\ \text { NN } & \text { Nested Networks } \\ \text { NS } & \text { National Standards } \\ \text { NZC } & \text { New Zealand Curriculum } \\ \text { PLD } & \text { Professional Learning and Development or Professional Development } \\ \text { NZ } & \text { New Zealand } \\ \text { NZC } & \text { New Zealand Curriculum }\end{array}$




\title{
CHAPTER 1 \\ The research focus, motivation and outline
}

\author{
"We may encounter many defeats, but we must not be defeated" \\ Maya Angelou
}

\subsection{Chapter overview}

This thesis explores principal leadership practice and how it intersects with the Low Socio-Economic (LSE) context to enable transformation. The pernicious and undeniable achievement gap (Bolton, 2017, OECD, 2015, Sturrock \& May 2002), and historically piecemeal nature of educational progress for students in impoverished contexts, is a deep-seated problem that Hallinger (2016) argues is multifaceted and interconnected, with no distinct origin and no definite solution. The existence of the achievement gap is not in dispute, but rather the education system's apparent inability to sufficiently address the gap. Entrenched in increasingly outdated concepts of leadership, the LSE principal leader must learn to advance through the challenges of $21^{\text {st }}$ century teaching and learning, to create transformational change, the type of change that is needed to address the achievement - equity dilemma. In this chapter, I outline the research focus and consider the New Zealand (NZ) context in which NZ primary school principal leaders work. The researcher positioning is explained and the chapter concludes with an overview of the thesis structure.

\subsection{Research focus}

The thesis is an investigation into how primary school principals in NZ, practise leadership in LSE contexts for educational success.

Decades of education reform in NZ have not been able to resolve the worrying correlation between socio-economic disadvantage and underachievement demonstrating that the achievement gap is persistent. The ongoing focus on underachievement is most evident when comparing annual education report documentation over time. The 1999 Compulsory School Sector in New Zealand report (MOE, 2000), showed a strong link between socio-economic status and achievement at school. Whilst primary school assessment data were not formally gathered to exemplify this correlation, it was noted that at secondary school level, students in 
"schools drawing from lower socio-economic communities were less likely to sit school certificate than those from schools drawing from higher socio-economic communities. The majority of papers sat by students from deciles 1-3 schools were awarded D or E grades" (p. 70). The report identified that while reasons for underachievement are wide-ranging and complex, low socio-economic status undoubtedly impacted. Thus, the report identified the need for a new strategic direction to raise the success of students such contexts.

Despite this call for a change of direction almost 20 years ago, the achievement gap has remained. The most recent 'Ministry of Education Annual Report 2017' (MOE, 2018) concedes that NZ is still plagued by high levels of disparity: "Many students attending low decile schools continue to get poorer outcomes from education" (p.9). Moreover, notes taken from the 'Tomorrow's School's Review, Independent Taskforce Meeting' (2018) indicate that at primary school level, many students are not receiving adequate support to address learning needs and this further increases the gap's ongoing and persistent presence.

While the achievement gap continues to be problematic, there is some evidence to suggest the gap may be closing. In the 2018 report 'Equity in education: Breaking down barriers to social mobility (OECD, 2018), the socio-economic gap in mathematics performance among 10-year olds, while the largest of England, Korea, NZ and the USA, showed that the NZ equity measure had improved. However, OECD reports suggest that such incremental changes are not leading to the sort of overall progress required to narrow the gap (OECD, 2016). Bolton (2017) believes "the vision of all children having access to a high-quality education that prepares them for success later in life, no matter their ethnicity, where they live, or how much money their parents make, is not yet a reality in New Zealand" (p. v). This suggests incremental change has not significantly altered the status quo, or the trajectory of success for LSE students.

The position assumed by the Ministry of Education (MOE) in NZ is to identify what is continuing to hinder LSE student success - a cohort historically made up of Maori, Pasifika and those learning English as a second language (Houghton, 2015). The NZ Education Secretary most recently labelled the underachievement of Māori especially, 
as chronic, intractable and systemic (Holsted, 2018), highlighting the issue of inequity in schooling.

Human resources appear to be the most popular lever for improving academic attainment. Researchers such as Leithwood, Jantzi and Steinbach (1999), Marzano (2005) and Robinson, Lloyd, and Rowe (2008) focus on leadership, second only to teachers, as paramount for students' success, and this is particularly relevant for students from disadvantaged homes (Pashiardis \& Notman, 2012; Shields, 2013). An OECD (2012) report on equity and quality in education states that "school leadership is the starting point for the transformation of disadvantaged schools" (p. 11). Since the principal is the most interconnected leader in any school, focusing on them may more obviously highlight how leadership practice can address barriers to student achievement. Education scholars Bezzina (2013), Day (2005) and Sergiovanni (1996) concur, agreeing that better principals create better schools. This research aimed to find out what principal leaders were doing to interrupt the cycle of poverty, rather than exacerbate it, and what expertise they drew upon in their specific contexts to address the systemic conditions they face.

While leadership practices that create conditions for improvement are vigorously documented, there is less written about how principal practice might occur in context and in turn, positively influence whole-system transformation. Robertson (2015) highlighted the importance of understanding practice, and suggests the "policy rhetoric is there - the practice not necessarily so" $(2015$, p. 16). What happens in LSE schools that reinforces or combats the current trajectory and the ability of leaders to come to grips with the complexity of their work is important to ascertain. Portin (2000) argues that the educational and social problems of students and the impoverished conditions of communities influence how leaders respond within their school culture. According to Thrupp (2010), such a context has been omitted from NZ research with Wright (2011) referring to leadership as 'bastardised', a regulating of leadership that is counterproductive to its effectiveness. A better understanding of the interaction between LSE context and leadership practice will provide a local contribution to the global commitment by leaders globally to reduce underachievement. 


\subsection{Research aim and objectives}

The research aim was to investigate how principal leadership and the school context interact to disrupt the current achievement trajectory. More specifically the research objectives are to:

1. recognise principal leadership practice as a complex system so that systemic solutions might be identified

2. understand the ways principals interact within context to encourage home-grown solutions to the achievement gap

3. identify the likelihood of transformational change when principal practice is viewed from within a more complex perspective.

This study has the potential to bring together leadership, context and measures of success in a way that provides new insights into the current understanding of a principal leader's practice to positively affect educational achievement, and ultimately, more successful life chances.

\subsection{The NZ context}

This section identifies the initiatives, frameworks and policies designed to confront the achievement gap. Appendix A also offers a chronology of relevant policy documents.

\subsubsection{Education reform}

Public sector and education reforms of the late 1980s shape the basis from which the system operates today. The report by a taskforce led by Brian Picot was the foundation for the Reform of Education Administration in NZ (Department of Education Taskforce, 1988). These reforms saw the compulsory education system redesigned so that substantial financial and administrative responsibilities moved from the state agencies to local schools. Boards of Trustees (BOT) ${ }^{1}$ were tasked with a variety of new responsibilities, and acted independently within national guidelines under a policy

1 The BOT is a governing school body (inclusive of the principal and a staff representative) who work on behalf of all stakeholders in the community and are accountable to the parents, community, and crown. 
that became known as 'Tomorrows Schools' (MOE, 1998). Responsibilities included setting and monitoring school targets, ensuring effective management of staff, recognising educational needs of students, and offering principals support.

The Tomorrows' Schools policy (Ministry of Education, MOE, 1998) had major and ongoing influences on the role of the principal. Promoted advantages of the policy were that leaders could more appropriately make decisions to reflect the diverse local community needs through the day-to-day running of the school. Niesche (2016) argues, however, that while favourable, the policy instead became a political and administrative force, and expanded the leader's responsibility for finance, property and human resources as well as pedagogical practice and community development. The policy's effect was therefore to increase the role of managerialism and created an accountability culture (Wylie, Burgon \& Cosslett, 2015). For a school principal driven by social justice, this often meant grappling to find the skills and resources that were needed to run a school successfully (Niesche and Keddie, 2016).

\subsubsection{Primary schooling}

State primary schools are well attended in NZ with only marginal competition from integrated and private schools. Most children aged 5-12 years attend NZ state primary schools $^{2}$ but may also attend state integrated schools ${ }^{3}$ or private schools. In 2017, there were 1,945 primary schools (Education Counts, 2018) ranging in size from 10 to 2,000 students, most of whom fell into either the state or state integrated categories above. Around $50 \%$ of primary schools have rolls of fewer than 100 , and up to $90 \%$ of schools are in cities (MOE, 2008). Both state and state integrated primary schools that are the focus of this research.

Recognition of the impact of economic status on school communities was acknowledged in the 1990s. The government's funding formula per school allows for: base funding by type and size of the school; per-pupil funding by year level; an approved teaching staff ratio; and targeted funding to recognise various other costs and provide additional assistance for those at risk of educational under-achievement (Blaikie, 2012).

2 State schools teach the NZC, are non-religious and are $90 \%$ funded by the state.

3 Integrated schools are schools that have a unique character and have become part of the state system. While they too teach the NZC, and are mainly funded by the state, they have their own set of aims and objectives to reflect their character and may charge a compulsory fee to parents. 
All schools were allocated a decile ${ }^{4}$, and this rating was written into most official documentation. A more recent report by the Post Primary Teachers' Association (PPTA, 2013) argued that the decile equation, which was intended as a way of targeting resources, did not produce adequate funding for those in need, indeed "Only in schools where almost all of the students are from the lowest socio-economic communities (decile 1) would the school profile be homogenous enough to be represented by a single figure" (p. 5). Because of such confusions, the ranking was removed from the Education Review Office (ERO) ${ }^{5}$ school reports in 2012, although the 'Ministry of Education's Education Counts Find a School' site continued to identify decile for longer. A risk index is currently under development to replace the decile rating system. The government intends that this will see additional funds allocated more accurately to at-risk students. Dependent on the consultation process, changes to school funding are programmed to occur in 2020. The LSE context was recognised as having a detrimental effect on student outcomes and required policy in the first instance to disrupt the cycle of underachievement.

School accountability measures indicating attainment are increasingly evident. Schools produce annual plans as part of the planning and review process. Strategic plans are created locally so that goals, inclusive of student achievement targets in literacy and numeracy, can be identified and measured. The plans are shared and reported on by the BOT to the MOE. A summative evaluation is done in schools every 3-5 years. Charged by ERO who reports publicly on the quality of education and care of students in all New Zealand schools and early childhood service centres. These reviews can be accessed online, and comment on the school background and context, key findings and recommendations for the future.

\subsubsection{Teaching and learning}

Various national documents underpin effective teaching and learning in NZ schools and are pertinent to the work of the principal leader. These include the New Zealand Curriculum (NZC) which was substantially reviewed and republished at this time (Ministry of Education, 2007). Supporting documents included: Ka Hikitia: Accelerating Success 2013-2017 (Ministry of Education, 2013); the Pasifika

4 A decile is a ranking system of all schools used by the MOE to determine the extent schools draw on students from LSE communities and ranges from the lowest score of 1 through to 10.

5 ERO is the government department that evaluates and reports on education in all schools and early childhood facilities across NZ. 
Education Plan 2013-2017 (Parata, Joyce, Clark, \& Benny, 2013); information on National Standards (NS), and teaching and leading codes of conduct (Education Council of New Zealand, 2017b).

The NZC has been the principal document used by primary school leaders since 2007 to set learning directions and to guide schools as they review their local school curriculum (Ministry of Education, 2007). The overall vision of the NZC is for, "Young people who will be confident, connected, actively involved, lifelong learners" (MOE, p. 7). Eight key values are thought to empower "people to live together and thrive" (p. 10). Additionally, the five key competencies, identified as fundamental to learning in a complex society, bring social and academic contexts together. Schools and principals have a certain amount of discretion in how they implement the eight key learning areas since curriculum interpretation relies on the place, people and expertise in the local area. Principles associated with high expectations, The Treaty of Waitangi ${ }^{6}$, cultural diversity, inclusion, learning to learn, community engagement, future-focus and coherence inform a school's vision, values and key competencies. The curriculum specifies five levels following young people's expected growth in levels of cognition as can be seen in Table 1.

Table 1: Curriculum levels relevant to students in primary schooling in NZ, adapted from the NZC (MOE, 2007)

\begin{tabular}{|c|c|c|}
\hline Curriculum level & Year level & Age \\
\hline 1 & $1-3$ & $5-8$ \\
\hline 2 & $2-6$ & $7-11$ \\
\hline 3 & $4-8$ & $9-13$ \\
\hline 4 & $6-11$ & $11-16$ \\
\hline 5 & $7-13$ & $12-18$ \\
\hline
\end{tabular}

6 The Treaty of Waitangi was a document signed in 1840, and is constructed on the principles of partnership, participation and protection. These principles underpin the relationship between NZ Māori and the Crown. 
It was accepted that many students do not fit this expectation of progress, since irregularities are created by those "with special learning needs, those who are gifted, and those who come from non-English speaking backgrounds" (Ministry of Education, 2007, p. 45). Nevertheless, the expectations do still act to set a benchmark for the progress of all students regardless of background.

Tensions around curriculum autonomy versus a wider expectation of high standards resulted in the introduction of the NS. In May 2008, the standards, for use with pupils from Years 1 to 8 (aged 5-13), set measurable benchmarks of primary pupil attainment and clear expectations and timeframes for achievement in reading, writing and maths (MOE, 2009). Assessments of students' academic achievements, NS were required until early 2018. A newly elected coalition government recognised the unintended consequences of NS which included increased competition, the pedagogical challenges associated with what the results were suggesting teachers needed to do differently, and the need to rebuild trust in the profession and direct attention to strengthening educational partnerships between parents, schools, and school communities.

Ensuring high standards required a greater focus on the two main sub-groups that made up the LSE cohort of concern. Indeed, over many years, there has been a growing effort to promote Māori and Pasifika success. Ka Hikitia, accelerating success 20132017 (MOE, 2013), was a plan developed to help fast-track changes to the education system so that Māori students could expect to gain the skills, qualifications and knowledge they needed to enjoy and achieve success as Māori. The three areas identified to make the most significant difference were quality leadership for teaching and learning, support by effective governance, and family engagement. Most pertinent to primary school leaders are three overall goals that aim to ensure that: all Māori students have access to high-quality Māori language education; Māori students have strong literacy, numeracy and language skills; and conditions are created for Māori to enjoy and achieve as Māori (Ministry of Education, 2013). The MOE has committed to specific actions in support of these goals, and these include: targeting resources to where they are most needed; offering a stronger voice to aiga/whānau ${ }^{7}$ and students; sharing effective practice; and developing further measures and indicators.

7 Aiga/ whānau are Samoan and Māori words for family. 
The Pasifika Education Plan 2013-2017 (Parata et al., 2013) highlighted the importance of improving outcomes for Pasifika learners. The MOE Statement of Intent included two priority outcomes: improving education outcomes for Māori and Pasifika leaders with Special Education Needs (SEN) and those from LSE backgrounds; and maximising the contribution to the NZ economy. The goal most applicable to primary schools was the goal that focused on Pasifika learners excelling in literacy and numeracy. Specific actions by the MOE included: responding to identity, language and culture, and adopting a Pasifika way of working that includes cooperative partnerships with families. Both plans focused on drawing on students' cultural identity to ensure more diverse perspectives of success were acknowledged. This expands the breadth and depth of a growth mind-set.

\subsection{Leadership}

Six essential policy documents and/or strategies helped to contextualise the NZ approach to school leadership and evidence growing recognition of the importance of building a community of leaders committed to a more collective response to education reform.

First, Kiwi Leadership for Principals (MOE, 2008) discusses the role of principals as "leading both the learning and organisation to enhance educational outcomes for all young people" (p. 4). The qualities, knowledge, and skills required to lead NZ schools have 'relationship' as a central focus. Four key components of practice include culture, pedagogy, systems, and partnerships and networks. Kiwi Leadership for Principals is the leading exemplar on which principal leaders are expected to model their practice, and highlights the skills and qualities of effective principal leadership within the NZ mainstream setting.

Second, quality outcomes for Māori require leadership which is culturally responsive. Leadership that is rooted in the idea that clearly must understand their own belief and values about cultures and people different from themselves, so they are able to effectively lead in multicultural settings (Terrel \& Lindsey, 2009) - a form of leadership critical in working with marginalised groups to address barriers to achievement. Rangatira (MOE, 2010) is a Māori Medium Educational Leadership report which presents a model of leadership that reflects some of the critical leadership 
roles and areas of focus in Māori medium settings. These roles include: He Kaiwhakarite, manager; He Kanohi Matara, visionary; He Kaiako, teacher and learner; He Kaitiaki, guardian; He Kaikōtuitui, networker; He Kaimahi, worker; and $\mathrm{He}$ Kaiarataki, advocate. These are woven together with seven key focus areas which include: placing the learner at the centre; spiritual and holistic wellbeing; recognising relationships as key to practice; the preservation of Te Reo Māori, the use of Māori customs and protocols; Māori discourse and knowledge; and the uniqueness of the school context. These key roles and focus areas combine to create leadership practice that contributes to high-quality educational outcomes for Māori.

Third, professional standards help improve upon practice. The principal professional standards in NZ focuses on: school culture, pedagogy, systems and partnerships and networks and:

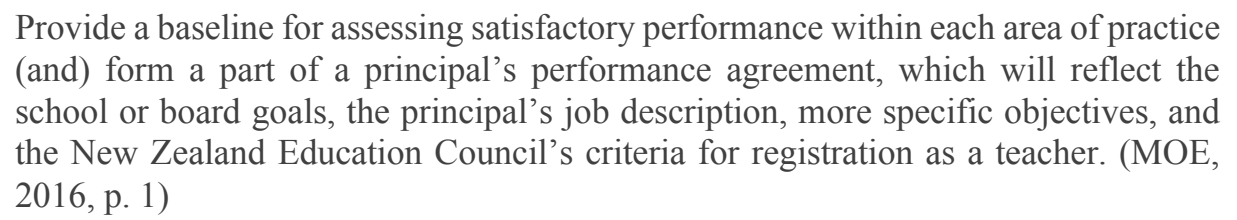

This standard set a baseline for leadership practice.

Fourth, the teaching profession of Aotearoa New Zealand (ECNZ, 2018) developed a strategy that will see education professionals advance their leadership capability. A systems-level approach will help to grow and build leaders at all levels, and identify investment priorities in leadership development. Four focus areas for the implementation of the strategy includes to: guide leadership practice and learning; clarify the capabilities needed for effective leadership across the teaching profession; personalise the professional learning required; and ensure a connected, encouraging and engaging leadership network.

Fifth, an educational leadership capability framework has been developed to support the intention of the leadership strategy (ECNZ, 2018). The capabilities are:

- $\quad$ building and sustaining high trust relationships

- ensuring culturally responsive practice and understanding of NZ's cultural heritage, using Te Tiriti as the foundation

- building and sustaining collective leadership and professional community

- $\quad$ strategically thinking and planning

- $\quad$ evaluating practices about outcomes 
- $\quad$ adept management of resources to achieve vision and goals

- attending to their learning as leaders and their wellbeing

- embodying the organisations' values and showing moral purpose, optimism, agency and resilience

- contributing to the development and wellbeing of education beyond their organisation. (pp. 6-7)

The capability framework gives an in-depth description of the nine capabilities, along with the varied ways they appear when leading an organisation, team or as an expert teacher.

Sixth, school leadership practice is evaluated by ERO using the published evaluation indicators (ERO, 2016). According to ERO, these indicators provide an opportunity to focus on what matters most in improving schools and outcomes for all our students, "Using indicators to improve practice in schools depends on skill in using data, creating cultures of inquiry, engaging in deep and challenging conversations about practice, and changing long-established beliefs and patterns of practice." (p. 6). Specifically, there are six leadership evaluation indicators that support equity and excellence and include: developing and pursuing the school's vision, goals and targets for equity and excellence; promoting and participating in teacher learning and development; ensuring effective planning, coordination and evaluation of the school's curriculum and teaching; building collective capacity to do evaluation and inquiry for sustained improvement; guaranteeing an orderly and supportive environment conducive to student learning and wellbeing; and building relational trust and effective collaboration at every level of the school community (ERO, 2016).

Together these six policy documents and/or strategies provide opportunities to develop cohesion between the knowledge and practices required to improve and evalute leadership.

\subsubsection{Professional learning and development initiatives for teachers}

There is growing recognition of the need for developing teacher Professional Learning and Development (PLD) that is evidence-based. A systematic approach adopted by the Ministry of Education to gather and promulgate research evidence to inform teaching, learning and leadership practice in schools, using an internationally reviewed methodology, has become known as the Best Evidence Synthesis (BES) series. These 
syntheses broadly assist leadership practice in schools and the design of PLD. The School Leadership and Student Outcomes: Identifying What Works and Why Best Evidence Synthesis, provides support for leaders by describing the characteristics of leadership that most improve outcomes for student (Robinson, Hohepa \& Lloyd, 2009).

Movement is occurring away from an individual school to a collaborative approach to education reform. The Investing in Education Success policy (MOE, 2015) was created in 2015 to help boost student performance and provide a more collaborative cross-school means by which to achieve this. It enabled highly effective teachers and principals who were paid a supplement to share expertise across a community of schools, for better student outcomes. On the one hand, the initiative acted as a catalyst and support for leaders to enact more effective practices, such as those outlined in the BES; and conversely it remains controversial since these positions elevate certain principal colleagues to an "expert" level and the source of all solutions. Developing incipient local leaders then becomes a priority. Emerging Leaders is a pilot programme due to be completed at the end of 2019 (MOE, 2017). It works with communities of learners $^{8}$ and focuses on developing emerging leaders across schools, not just those in formal leadership positions. Potential leaders are identified and help to lead aspects of change in the community, develop professional inquiries and seek local solutions. Expert partners, work with the leaders in the role of coach, helping their leaders to define problems, gather and critically analyse data, and implement evidence-based actions to accelerate student achievement.

There is a focus on promoting culturally responsive leadership. He Kākano (ERO, 2010) was a significant in-depth professional learning programme with a focus on leadership practices that took account of the culture of Māori learners and built relationships that result in more significant achievement. This national strategy included aspects of coaching and mentoring and worked to support and develop the inherent capabilities and skills of Māori students, and acted as a framework for matching leadership goals to the unique NZ context. Although its success is welldocumented (MOE, 2013), funding for the programme has ceased, although the material is still available for use by schools.

8 A community of learners is known in Māori as Kahui Ako. 
Timely professional learning and development, with a greater focus on evidence-based practice, more collective learning opportunities, and improved cultural responsiveness, has the potential to encourage greater alignment of teaching, learning and leadership.

\subsection{Future direction}

In addition to the aforementioned initiatives, at the time of completing this thesis, it was decided by a new government, that a significant overhaul of the education system to refocus on the next 30 years was required. The government organised the education manifesto into four priorities and with the learner at the centre (Labour government, n.d). Priority one is barrier-free access and will include more in the school's annual budgets, so parental donations can cease. Priority two is quality teaching and will focus on guaranteeing adequate teacher supply, strengthening teacher leadership and collaboration and raising the standard for teacher entry. Priority three is quality public education and aims to put education at the heart of communities, making sure schools are modern and well equipped. The last priority focuses on competencies, making the best use of technology and transforming career advice.

Multiple reviews to deliver on a 30-year strategy have been set in motion, from education policies in the early childhood sector through to the tertiary sector that were inclusive of education design and testing measures. The Tomorrow's Schools Review report (Tomorrow's Schools Independent Taskforce, 2018) was recently released and focused on governance, management and administration of schools. The report signalled a need for change in the way education is organised. Eight key issues were identified to address the serious inequalities and excellence issues in NZ. The first is governance that requires a greater sharing of professional support and less competition between schools, which has become especially detrimental to schools in LSE areas. The second is schooling provision. It is recommended transition between schools be addressed along with better support for more Māori language provision. The third is school choice. Unhealthy competition needs to be reduced and biased enrolment schemes dismantled. The fourth is greater disability and learning support. The fifth is to improve the quality of teachers and the sixth the quality of school leaders. The seventh is connected to resourcing in support of greater equity between schools. Lastly, central government agencies of the MOE will be called on to develop 
assessment, pedagogy, curriculum and learning. Combined, these eight areas are hoped to prompt the change required to address inequality and excellence.

The direct link between holistic wellbeing and the future of NZ is being made. The NZ government has recently adopted a Living Standard Framework (LSF) (Ng, 2018), the details of which bring together economic, social justice and ecological concerns. The LSF is based on four capitals: natural capital, referring to the state of the natural environment; human capital, referring to the skills, knowledge and wellbeing that enable people to participate fully in life; social capital, encompassing the norms and values that underpin NZ society; and financial/physical capital, consisting of physical and financial assets. The approach will help the government identify opportunity and risk associated with sustainable growth in all four areas $(\mathrm{Ng}, 2018)$. As a framework in use by government, the LSF, could also underpin educational success in schools. Success that is defined as future-focused, is mindful of not just academic, social and economic outcomes, but environmental outcomes also.

Any future changes it seems, are intended to support significant transformation of the education system rather than simply continue the current trajectory of incremental progress.

\subsection{The researcher}

My early life in an LSE environment parallels the research context and student participant experiences. My parents, despite minimal education, worked hard in the 1960s and 70s to successfully raise four children. My father was often out of work during the latter period of the economic recession. Limited alternative employment was available because of his under-developed literacy capabilities. We did not own a vehicle, and our house was inadequately heated. Due to financial constraints, learning a musical instrument or attending dance classes was not possible until I could fund these in my teenage years. Still, our family of four children were all cared for and loved. Both grandmothers baked and knitted for us, and our uncle who managed a shoe factory, provided shoes at Christmas. In the neighbourhood, there was a collective response to limited resources through trading produce and labour. My family successfully navigated the LSE context in these varied ways. 
My years at primary school were positive. I was conscientious, enthusiastic, felt a real sense of belonging, and was academically successful. When I attended secondary school out of the area however, it became apparent to me that my origins created a divide. School friends described my neighbourhood as rough and undesirable, and making friends was not easy. Upon my completion of secondary schooling, my father expressed his expectation that I go straight into employment. No-one in my family had ever attended university, but I believed that tertiary qualifications would assist me to fulfil my dream of travelling the world. So instead I enrolled at Teachers' College.

My years of teaching have also shaped the topic of this thesis. An interest in the educational achievements of LSE communities evolved out of my first teaching position in the Porirua basin in 1990, a context considerably affected by material deprivation. I worked in a mostly Pasifika and immigrant community. As a newly qualified teacher, I wondered what difference it made that my students lived in damp homes, had ongoing health issues, were often sleep, and food deprived, and had complex family lives, missing school to stay home to look after their younger siblings. My challenge was to cater to learning needs within this context while meeting learning expectations with a 'no excuses' mentality. Support from my principal was lacking. The outcome of this experience made me question the impact that a good leader might have had on my teaching while supporting students and their families in material hardship. This research attempts to belatedly answer this question.

I align my beliefs about teaching and learning with Vygotsky's (1979) socio-cultural approach which states that realities constructed occur between people and that learning is naturally collaborative. Additionally, Dewey (2004) states that learning is an experimental process and best achieved through a hands-on approach. Both Vygotsky and Dewey focus on learning as a process, and this notion of developmental thinking aligns with my belief that learning and teaching are often about experimenting with others to develop knowledge. Since learning is a social process, it is vital in my opinion, that learning communities be inclusive.

Practice roles in my educational career have been numerous and have included: teacher, Resource Teacher Learning and Behaviour, deputy principal, Head of English, Head of Primary, and a university tutor and teaching fellow. It was as a consultant working with a variety of LSE schools that had been identified by the MOE as having 
below-standard reading/writing results where I finally saw the value of effective leadership. I worked as a facilitator joining school principals and literacy-led teams to inquire into leadership effectiveness when working on school-wide literacy and numeracy targets. Working primarily from the BES leadership for learning model (Robinson et al., 2009), team/s experienced a varying degree of success in narrowing the achievement gap for target students. Some leaders could show their work narrowed the achievement gap considerably, while others could not, and fewer still were able to show sustainable progress. Witnessing such variable outcomes suggested that the approaches were piecemeal, perhaps not because leaders did not know what to do but instead did not always know how to match theory to practice from within the complex settings within which they worked. I am interested specifically in unpacking the influence of context on leadership and equally a leader's influence on context.

\subsection{Overview of thesis structure}

This thesis examines how principals' leadership practice promotes educational success. Chapter one has outlined the correlation between LSE status and the educational achievement gap and why such a perennial pattern of underachievement is important to interrupt. Outlined is the journey the MOE has taken towards solving this prevailing, complex problem, from within the broad NZ context. My interest in this topic has also been explained.

Chapter two is a narrative literature review providing a critical analysis of leadership through consideration of the emerging themes that make up educational leadership, including: positionings, purposes, attributes, effectiveness, and context, and the implications of this on the principal leader. Educational success is defined and areas identified where there is need for more empirical research mindful of the context within which principal leaders practice.

Chapter three gives details of the theoretical framework, employing Bronfenbrenner's (1979) ecological systems theory of child development, complexity theory, and Capra's (1997) 'Systems View of Life'. Since social phenomena do not occur in a vacuum but rather are embedded in ecosystems, layered together these theories offer a practical and fitting research framework. 
Chapter four offers an overview of the methodology which grounds the study in a constructivist/transformative approach to the formation of knowledge. The choice of an embedded, emergent case study is explained, along with the purpose, rationale, method, and study trustworthiness.

Chapters five and six describe and explore in detail, the findings in relation to the two units of analysis of the case study. Details are related to the individual sites and principal leader and then the who, what, how and why of the principal's leadership network. These chapters conclude with an analysis of feedback loops seen in action and what these indicate.

Chapter seven includes both a cross-case analysis and discussion. In bringing the two units of analysis together, similarities and nuanced differences are studied. I conclude the chapter by highlighting the essential characteristics and overall network capabilities.

Chapter eight summarises the findings, draws conclusions from them and indicates what is needed for system-wide reform if principal leadership practice is to aid transformational change. Limitations of the study are identified and suggestions for further research in this field are suggested.

\subsection{Chapter summary}

This introductory chapter has provided a context for this research inquiry into principal leadership practice in LSE school settings for improved student success in the New Zealand schooling system. The research focuses on the intersectionality of leadership and context. I have argued that localising and widening the problem will help better understand the interrelationship between complexity and leadership as a lever for reform and transformational change. Background information on the significant aspects of NZ education reform from the past, present and the future trajectory undertaken were provided. The researcher's background concerning the context of this study has been explained and an overview of a theoretical framework presented. Finally, the structure of the thesis is outlined. 


\section{CHAPTER 2 \\ The literature review}

"There are things known and there are things unknown, and in between are the doors of perception" Aldous Huxley

\subsection{Chapter overview}

The purpose of this narrative literature review is to identify key themes from dominant educational leadership theories and approaches relating to emerging concepts of educational leadership, and major findings from empirical studies relevant to the research scope. The implications of these findings for principals will be discussed and any gaps in the literature that have not been sufficiently investigated identified. Together the theories and approaches show a shift from leadership as a largely theorybased phenomenon to leadership as a phenomenon of practice. Four themes emerged from theoretical readings and empirical studies. Theme one is 'positioning' showing the shape of leadership, who is involved in leadership and how power is shared. 'Purpose' is the second theme which investigates the why behind leadership practice, from the deeply held personal beliefs of a leader to broader education and even wider societal considerations. Third is 'behaviour' which considers how the leader interconnects and self-organises with others so practice is cohesive. The fourth theme, 'progress', outlines the types of positive outcomes that can be expected. This theme includes summative and formative assessment measures and systemic improvements. The merging of these themes identifies leadership practice as a coupling of and/both concepts. All four themes are then discussed with the LSE context central to the discussion. Defining educational success completes the main body of the review to help create a better understanding of the varied definitions behind the term. The chapter ends with the identification of gaps in the literature that present a convincing research problem for further investigation.

\subsection{Contextualising educational leadership}

Ideas about leadership have evolved over time, the beginnings of which were established in business psychology. Educational leadership took a similar course, emerging as a public administration role (Hulpia, Devos, \& Rosseel, 2009), whereby leaders in schools were expected to influence others to achieve goals and enforce rules 
to maintain set standards (Bush, 2008). This modern concept of leadership was derived from societal structures from the past.

Scholars, including Sergiovanni (2003) in the USA, and Fullan (1982) in Canada, began to rethink traditional notions of management theory alongside education reform, and revolutionised the concept of leadership in education. This work considered concepts related to change, professionalism, and learning. Fullan's ground-breaking work on the meaning of educational change, connected leadership with the change process through concepts of collegiality, capacity building and communities of learning. Meanwhile, Day (2005) and Sergiovanni (1996) evolved the idea that moral leadership would improve the 'right' things (p. xiii), or competence and virtue so professionalism thrived. Elmore (1979) was instrumental in seeking a shift from the business model of leadership to a purely education-focused model, one in which the act of learning was paramount. Bush (2010) argued that without a learning focus there was likely to be greater prominence placed "on procedures at the expense of educational purpose and values" (p. 391). Educational leadership therefore became focused on developing learning organisations that were both socially and educationally responsible.

A summary of the literature analysed in this review can be found in Appendix B and demonstrates a representative sampling of approximately 26 years of thinking on the topic.

\subsection{Themes of educational leadership practice}

Rather than a checklist of segregated practices and theories, this literature review is a high-level condensation of main themes evident in the literature as they applied to LSE settings. The development of the concepts in the literature, progresses from a simple idea that leadership is a clear influential consequence of the leader on the follower, to a more complex idea of leadership that exists in an emerging space, and in which context and people are in constant interaction with each other.

\subsubsection{Leadership positioning}

Leadership positioning is the first theme evident in the literature and traces the way the notion of leadership as a phenomenon has evolved. Initially understood as a role, it has since been described as a group activity, and more recently as a pattern of 
practice. As such, a leader may engage authoritatively, collaboratively or interdependently.

Role

Leadership has been referred to in the past as an authority held by one person with influence over others (Hohepa \& Lloyd, 2008). This type of leadership is variously labelled as authoritative (Wafula, 2015), transactional (MacNeill, Silcox, \& Boyd, 2018; Shields, 2013), or trait (Zaccaro, 2007), reducing leadership to what Hiller, Day, and Vance (2006) refer to as expert, Wheatley and Frieze (2011) as hero, and Pepper (2010) as authority. Such positionings recognise how leaders engage with followers and influence culture and systems (Wafula, 2015), and conversely, the various perceptions that followers have of their leaders (Mac Neill, Silcox \& Boyd, 2018).

The notion of leadership as a role implies that power lies primarily with the person in the leadership position and treats the follower and leader positions as distinctive (Hiller et al., 2006). Leaders may make significant decisions and expect others to obey or alternatively may delegate power. Blanchard and Broadwell (2018), Crippen (2004) and Portelli and Campbell-Stephens (2009) refer to leadership that is guided by the dignity and rights of others, as servant or moral leadership.

Positioning leadership as a role creates benefits and barriers to improving student achievement. Benefits include a way of acting that is, according to Sergiovanni (1996), likely to positively influence others. Barriers include a heavy reliance on the leader to effect change, situating the responsibility for growth in the designated 'other' (Robinson, 2004). According to Lambert (2003b), this leaves teacher and student capabilities largely underutilised. Firestone and Riehl (2005), moreover, suggest that leadership as a role does not encourage creativity from any member of the team who might have sound ideas, creating a more mechanistic approach with little room for the discovery of latent talents. Bush (2010) also argues, that leadership as a role may encourage a misuse of power, with followers being compelled to make choices that go against their beliefs.

A principal's role is diverse. Walker, Sorenson, Smaldino, and Downey (2008) divided the role into four basic tasks: those of being a business manager involving technical managerial skills, a leader of people involving human resourcing skills, a curriculum 
developer involving technical-educational skills, and a change agent involving speculative-creative skills. Identifiable within the literature review are two main tensions that exist for principals.

A first tension is the large and ever-increasing workload. According to Eckman \& Kelber (2010) multiple responsibilities affect a principal's job satisfaction and their ability to cope with both personal and professional obligations. While Grenda and Hackmann (2014) and Notman (2011), state that a leader's work can become fragmented and time-consuming, Rice (2010) also adds that the manageability of the role is dependent on the attributes of the leader, efficiency of the network, and the type of problems being addressed. A recent NZ report on 'principals and their work' (Wylie, 2016), similarly describes the way principals manage their time as highly variable and dependent on such things as funding, staffing, accountability measures, and competition between schools. Furthermore, with $42 \%$ of new principals indicating high or extremely high levels of stress, the principal leadership workload in NZ is in urgent need of revision (Wylie, 2017) and is likely unsustainable in the longer term.

A second main tension highlighted in the research is the need to balance leadership work and management responsibilities. To serve teachers and students, schools require sound management systems, but also, professional knowledge and expertise to help improve teaching and learning (MOE, 2008). As the workload increases, assigning time for both pedagogical change and management tasks can be difficult to resolve and may affect the leader's ability to focus on teaching and learning (Gronn, 2003; Notman, 2011). Moreover, in NZ, schools are self-managing and principals are not only managing and leading but also participating in school governance. This adds further to a principal's responsibilities.

\section{Group activity}

Considering leadership as a group activity emphasises the interactions among group members, offering a way to counteract workload concerns. Leadership as a group activity has come to be extensively accepted in educational research as democratic (Woods, 2005), shared (Kocolowski, 2010), distributed (Chatwani, 2017), or collaborative (Chatwani, 2017). These types of positionings demonstrate a nonlinear transaction between people (Lewin, 1976). Fullan (2006) considers this type of leadership as that which establishes a 'critical mass' and Spillane, Camburn and 
Stitziel Pareja (2007) call it a 'stretching' of responsibilities. Leadership as a group activity treats the follower and leader positions as interchangeable.

When leadership is viewed as a group activity, power becomes a process of social interaction. Relationships are at the centre of leadership since schools are "intrinsically a social enterprise” (Bryk, Sebring, Allensworth, Luppescu, \& Easton, 2010, p. 119). This reciprocal notion of leadership lessens the power differentials and offers all participants the opportunity to contribute to purposeful activity. Tasks are owned by the group (Barrera-Osorio, Fasih, Patrinos \& Santibanez, 2009), and decision-making and problem-solving are shared (Shields, 2013; Wheatley, 2006). Sebastian, Allensworth and Huang (2016) suggest that group leadership shifts thinking about who can learn and who can lead to a more fluid interaction between the two. This type of power is associated with mutual influence.

Leadership as a group activity is both advantageous and disadvantageous. Working collaboratively can bond teams (Sebastian, Allensworth, \& Huang, 2016), develop trust (Spillane, 2006), lessen unrealistic expectations (Jones, Lefoe, Harvey, \& Ryland, 2012), draw on individuals' strengths and skill development for greater reciprocity (Grenda \& Hackmann, 2014), and develop cooperative organisational structures (Gronn, 2009). Sergiovanni (2003) and Spillane (2006) also add that shared leadership motivates staff, and offers a clear convenient management design for completing tasks. Spillane et al. (2007) and Timperley (2011) contend that when leaders and followers engage in this way, the wider community is more easily included. Leadership as a group activity encourages engagement. Yet, criticism of leadership as a group activity is also evident in literature. Since schools require substantial participation by many to sustain the workload, the complexity of educational leadership may thus, be difficult to realise (Harris \& Spillane, 2008). Moreover, strong but flexible structures that allow for shared leadership are proving difficult to design (Harris \& Spillane, 2008).

Principal leadership as a group activity sees the principal informally facilitate their work to include others. Harris (2009) is clear that a flatter-type leadership structure moves collective responsibilities across organisational boundaries. A mixed methods longitudinal study by Spillane et al. (2007) provided evidence of this, showing that the daily workload of the principal includes involvement from multiple actors performing tasks for and with the principal. 
Principal leadership in NZ is a widely-shared activity amongst teaching staff. A report by Wylie, McDowall, Ferral, Felgate, and Visser (2018) gave the first national picture of teaching practices, school practices, and principal leadership and found that when working on administrative, curriculum, or PLD tasks, involvement from others was common. However, the results of the online survey of 403 schools providing a "nationally representative picture from 4,355 teachers, 335 schools and 353 principals" (Wylie et al., 2018, p. 1) showed that 47\% of teachers thought principals worked well with others to solve problems, and only $29 \%$ believed the principal developed leadership capabilities in others. These findings suggest that while teachers collaborate with principals on various tasks, few believed that they were being encouraged to show leadership.

Principal leadership is alternatively positioned both as a role and a group activity. Klimek, Ritzenhein and Sullivan (2008) and Capper \& Young (2014) posit that principal leadership is not an either/or choice between role and group but and/both. An integrative framework developed by Friedrich, Griffith, and Mumford (2016) described leadership as a collective pursuit but interestingly did not eliminate the role of the focal leader. O'Donoghue and Clarke (2009) and Friedrich et al. (2016) argue that both, individual leaders and the collective, influence organisational effectiveness, and advocate for combining the traditional notion of educational leadership alongside more contemporary models.

\section{Pattern of practice}

Leadership as a pattern of practice is more concerned with the patterns constantly being created by non-linear interactions. This type pf leadership is variously referred to as leadership as practice (Raelin, 2016), systemic leadership (Despres, 2004), constructivist leadership (Lambert et al., 2002) or adaptive leadership (Heifetz, Grashow, \& Linsky, 2009). Denis, Langley and Rouleau (2010) highlight leadership as a set of behaviours that are systemically influenced, fluid and materialise over time. As behaviours are modified, the learning occurring produces patterns. Leadership patterns are "not an external force but an integral part of the leadership dynamic" (Lambert, 2003a, p. 9) with the greater focus on the collective movement occurring in context. Leadership as a pattern of practice helps bring learning and context together. Patterns of practice identify leadership as more complex than previously realised. Raelin (2016) proposes that "in the twenty-first century organisation, we need to 
establish communities where everyone shares the experience of serving as a leader, not sequentially, but concurrently and collectively" (p. xi). It is likely that focusing more on patterns rather than outcomes will allow for this.

Engaging in learning for a collective purpose requires not power, but empowerment. Capra (1997) and Wilson, Cummings, Jackson and Proctor-Thomson (2017) propose empowerment as the best way of mandating mutual learning. Morrison (2010) suggests empowerment allows for greater trust and freedom. Ferriter and Cancellieri (2017), for example, refer to leadership that works to empower students rather than engage them. Along these same lines, Raelin (2016) refers to a type of agency that circulates - or an ability and authority to act. This type of power helps leaders create conditions for new patterns or emergence ${ }^{9}$ to occur rather than giving direction (Stone $\&$ the Centre for Ecoliteracy, 2009). Individuals who are trusted and confident, mutually influence those around them and form strong bonds. In a recent survey in America by Louis and Murphy (2918) leader and teacher were investigated to see if they had an impact on organisational learning. It was found that principal trust was directly related to teachers' perceptions of principal caring, an indication that in doing so, principals could foster equity, organisational learning and student achievement. Leaders who support a high trust context are likely to have safe and supportive schools.

Positioning leadership as a pattern of practice includes many of the advantages of leadership as a group activity. In addition, it encourages questioning and rewards innovation to ensure its own sustainability (Capra \& Luisi, 2016). As patterns are identified, the influence of the network widens to ensure the people and resources required to meet future growth needs are regenerated. This finding is in harmony with Raelin's (2016) idea that matching members of a learning community to contextual needs positions leadership as systemically influenced by organisational behaviour and involves an acceptance that leadership is somewhat fluid and emerges in motion. Klimek et al. (2008) claim that this means the organisation is more likely to have the commitment and energy to sustain it through difficult periods. Leadership that is viewed as emergent is focused on building adaptive capacity.

9 Emergence is used in this thesis to describe new structures or new behaviours that occur unexpectedly. In a complex adaptive system emergence occurs through endogenous selforganisation by the actors themselves rather than through any externally directed forces. 
Many of the negative aspects relating to leadership as a group activity are relevant here. Two aspects specific to leadership practice patterns are evident in current literature. The first is the difficulty with understanding leadership as a concept since pattern is constantly moving. Ongoing and rapid changes leave some members unclear about plans and future direction, offering only a vague pattern boundary, and less stability overall (Duignan, 2012). The second is the challenge of researching this type of leadership. Studying leadership as practice is "far more process orientated ... and requires slowing down the action sufficiently enough to study practices and interactions" (Raelin, 2016, p. 218). In a report by the University of Manchester UK (2011), the focus on emerging patterns of school leadership identified three important stimuli for change, "local dissatisfaction with current arrangements and/or opportunities for improvement; individual drive and vision at school level; and significant local acts of philanthropy" (p. 2). These findings, while useful to this study, indicate a focus on outcomes of practice patterns, rather than the identification of the patterns themselves. The need to focus on practice patterns has been highlighted but there is minimal guidance on how to do this (Fitzsimons, James, \& Denyer, 2011; Kempster, Parry, \& Jackson, 2016). To study leadership patterns of practice will require innovative techniques.

\section{Implications of positioning}

This section suggests that the more fluid the positions between leader and follower, the greater the levels of engagement within the wider organisation. Principal leadership practice, however, involves operating as both a leader and follower, since it involves the work of many, but also a formal position where power is constantly renegotiated and redistributed. Principal leadership is rarely explored or even referred to as a pattern of practice in the literature but this alternative reference offers the possibility of bringing leadership and context closer together. Many unanswered questions remain about what leadership is and how to study the phenomenon when viewed as a pattern of practice.

\subsubsection{Leadership purpose}

Leadership purpose is the second theme evident in the research literature. The purposes behind leadership are many, starting with personally held beliefs and values (Tubin, 
2017), to a shared vision (Bogotch \& Shields, 2014), to wider macro viewpoints (Fullan, 2011). Breadth of purpose requires a leader who can link their own identity with wider ideals so that more varied opinions are represented.

\section{Personal beliefs and values}

Personal beliefs and values lay a foundation for a leader's personal framework of influence (Tooms \& Boske, 2010) or leadership identity (Tubin, 2017). A belief is the acceptance of something as truth and guides action or "a way of being in, and knowing about the world" (Thomson, 2009, p. 1). A study in USA by Brackins (2012) used a survey to determine if leaders' self-reported actions were related to their beliefs and found actions were strongly related to beliefs in successful leaders. Personal constructs are therefore influential, and as noted by Tubin (2017), help the leader display a clear vision of themselves as a leader. Parrett and Budge (2012) go further, suggesting that to commit to leadership work in LSE contexts, leaders must not just influence others but "aggressively confront entrenched, counterproductive strategies and beliefs" (p.70). Goleman and Senge (2014) concur suggesting also that the value of self-belief, enables leaders to remain motivated to make difficult decisions, commit to improvement, and willingly try new ideas. Personal frames are therefore a useful tool for grounding personal ideas.

Values are defined by Nieuwenhuis, Beckermann and Prinsloo (2007) as "abstract internalised conceptions of what is important, and direct how you behave in a certain situation" (p. 69). Strong personal values are required for equally strong practices (Hallinger, 2011). Kekana (2013), in a study in South Africa, looked at the values of school principals from effective schools and found them, in comparison to ineffective principals, to be inner-directed. This meant that they were both assertive and had selfefficacy, believing that they could influence change. In the NZ context, Bishop (2008) has recommended leadership which includes the following central values and ideas: manaakitanga (leading with moral purpose), pono (having self-belief), ako (being a learner), and awhinatanga (guiding and supporting). These values support a leader's ability to be responsive to a diversity of needs in the NZ setting. Strong values are not only likely to be aligned with action but also develop the expectation of success.

A leader's beliefs and values are noted in the literature as inspiring various outcomes. Firstly, they are seen to influence others. In an Indonesian study aiming to identify the 
influence of a principal's personal support towards teachers' work, Asri and Tahir (2014) found that honesty and compliance were key values that significantly influenced culture, which in turn had a strong influence on organisational success. Additionally, Marcketti and Kadolph, in a large-scale longitudinal study (cited in Berson \& Oreg, 2016), confirmed that a relationship existed between what leaders valued and the development of children's beliefs and values. Both studies suggest that a leader's beliefs and values create a foundation for influencing others' personal philosophies and change efforts.

Secondly, beliefs and values helped to balance accountability stressors with practical needs. In research by Budge (2010), school and community leaders' beliefs about standards-based reform were explored and the influence on one local schooling rural community in America was investigated. The study used interviews with 11 community and school leaders. Participants were found to engage in both serving local interests and satisfying wider mandates, showing a concern for the place they inhabit not just the state and federal policy requirements they encounter. The conclusion explored critical place-consciousness as a possible tool to prioritise supporting individual student success and the beliefs and needs of their communities through an awareness of place. Endeavouring to improve the quality of life for communities living in a specific place is also advocated for by Jackson (2018). Second only to beliefs and values, a leader's context is highly influencing of which day-today stressors are prioritised.

Thirdly, research identifies that beliefs and values are important considerations when change is likely. As workload becomes more complex, it is thought that beliefs and values allow leaders to make focused decisions (Branson \& Gross, 2014). These authors refer to leaders using a moral perspective not a problem-solving perspective when making choices (Branson, 2014). Identifying one's position on an issue, making an informed choice and then seeing the decision through to a principled stance is identified as moral literacy (Branson, 2014). A moral compass, is an important decision-making tool required for effective change management.

Finally, evident within the literature is the potential connection between a leader's identity and values-based contingency leadership. In a case study of two NZ school principals and significant others (Notman (2017), it was found in the first year of a 
three-year study, that both principals brought "a distinctive set of values and strategic vision and belief systems by direction, work and deed" (p. x). Both leaders held a set of core personal values, and a growing professional identity which showed evidence of adaptive leadership.

With little empirical evidence to call upon, what is still unclear is the various ways principal beliefs and values inspire student outcomes in a NZ LSE primary school setting.

\section{Shared vision}

The school vision is often based on what the learning community hope to achieve. The vision provides guidance for school leaders and teachers, students and the community, but is also driven by history, context and people (Bogotch \& Shields, 2014; Day, 2014; Harris, 2003). A vision very often includes designing, evaluating and coordinating the curriculum, and developing and maintaining evaluation and inquiry (Eliophotou Menon, 2014). As such, leaders work to create conditions for improved student attendance and behaviour, increased student motivation and engagement, and a developed sense of responsibility for learning. Building a shared vision ensures a unified understanding of what is to be achieved and is described as a bonding exercise between leader and followers for a common cause (Sergiovanni, 1996). Pejza (1985) states that "without a vision to challenge followers, there's no possibility of a principal being a leader" (p. 12). A school that uses data to both set a vision and decide on where next to and how to get there, is argued by Parrett and Budge (2012) as an essential function that works to move the underachievement of students in poverty. The importance of the school vision for setting the collective direction cannot be overemphasised.

A shared vision ensures actions that work towards the development of a shared culture. School environments are hugely diverse, presenting a challenge for educational leaders as well as school staff who need to adjust their practices to meet the demands of the varied backgrounds, languages and ethnicities of the students in their care (Pejza, 1985). Having a school "become a powerful interconnected learning network" is something Caldwell (2005, p. 193) refers to as the lateral transfer of knowledge. This occurs when learning is transferred from one context to another. Johnson (2012) describes a culturally responsive leader, as one who includes the stories, values and 
cultural knowledge of a student's background in school curriculum. Bolstad, Gilbert, McDowall, Bull, Boyd and Hipkins (2012) suggest that to advance reform initiatives, leaders need to work with a diversity of stakeholders and include varied ways of being and knowing, so one culture does not preside over another. The work of a culturally responsive leader is to synthesise the many visions into one.

The development of wider perceptions of the school are also found in the literature. Miller (2018) suggests that in the face of budget cuts and demanding policy initiatives, school leaders are being forced to think in more market orientated ways. His findings on the nature of school leadership are derived from 16 countries and 61 school leaders, and while he acknowledges that schools are a place for social development, due to increased competition, they also require leaders to be entrepreneurs showing their schools as places of undisputed learning. Miller argues that this leadership type, which is highly influenced by national culture and context, and is approached differently by both males and females, will become more firmly embedded into the daily work of school leaders, regardless of country of location, school size or type, and a school leaders' natural ability towards or forced compliance with, entrepreneurialism. Viewing schools as quality leaning institute requires ongoing and deliberate consideration.

Awareness of broader issues

Day (2005), Fullan (2011), and Woods and Roberts (2018) advocate that leaders must learn to be aware of the big-picture and encompass a variety of wider purposes to their work.

The future of the economy has a political influence on leadership in schools. Pink (2000) argues that the current 'conceptual age' requires a move from an industrial labour-force to a knowledge economy. A knowledge economy is one that is "directly based on the production, distribution and use of knowledge and information" (OECD, 1996, p. 7). Pink (2008) maintains that new ideas are needed to create scientific and technological progress and to solve the unprecedented ecological and social issues currently occurring. Empathy and an ability to care for others, develop inventive and creative solution-finding, make meaning, develop big-picture thinking and organise patterns, are some of the skills required to thrive in complex times. Woods \& Roberts (2018), contend that leadership is needed that develops "relational freedom, self- 
determination, and critical reflexivity as the driving aim of distributed leadership by teachers, students and others in non-positional leadership roles" (p.1). That is, leadership that focuses on disseminating the will and skill to lead among all others. Fullan, Hill and Crévola (2006) claim that this means leadership in schools must aim to educate not $50-60 \%$ of students who naturally succeed in the school system but $90 \%$ or more. Developing learners for a knowledge society is an important purpose of educational leadership since student's outcomes heavily influence their later career choices and earning power.

Leadership development is therefore also concerned with enabling all citizens the right to fully participate and succeed in their lives. Social justice is a topic growing in momentum in the leadership literature, with Shields (2013) arguing that as the diversity of student backgrounds and abilities increases and the environments in which they live become more complex, a broader social justice approach to teaching and leading is needed to ensure no rights are excluded. However, social justice is currently construed as a "supplementary effort because of the power structures in society" (Tooms \& Boske, 2010, p. 283). Niesche (2016) argues that this type of leadership is not only for those in disadvantaged schools, as advocated for by Day (2005), but is the responsibility of all educators to establish environments that enable teaching and learning for all. Santamaría and Santamaría (2012), Shields (2013) and Theoharis and Scanlan (2015) concur, recommending that social justice be woven into everyday schooling through the development of socially-just curricula, pedagogies, programmes and policies. As schools and school communities continue to diversify, social justice leadership becomes even more important.

Recent NZ literature supports the international claim that social justice leadership is the responsibility of all. McNae, Morrison and Notman (2017) present ten case studies conducted in a variety of educational high-needs settings across NZ to explore how social justice leadership is enacted. One conclusion was that in NZ, high-needs cohorts were evident in a much broader variety of contexts. Therefore, all principals needed to be aware of the increasing inequities emerging in their schools and communities no matter their socio-economic rating. Social justice adds a further layer of complexity to leadership for consideration. 
Closely aligned with social justice, ecological concerns are also a pressing international concern. The earth is experiencing a previously unseen set of issues, bringing sustainability of life on earth into question. This crisis requires a type of mindfulness (Cordon, Brown, \& Gibson, 2009) or mana wairua ${ }^{10}$ (MOE, 2010) so that all living beings are viewed as members of ecological communities bound together in a network of interdependencies. In the field of leadership, this is sometimes referred to as sustainable leadership (Oghenejobo, 2014). Sustainable leadership, while inclusive of social justice, requires a mindful contribution to whole communities. Hannon and Peterson (2017) point out that schools therefore need not only change for improvement but be "reinvented as a key part of learning eco-systems; webs of civil society institutions powerful enough to enable humanity to address the problems which both threaten it and offer spectacular opportunities" (p. 13). Leadership in schools must be mindful of wider ecological concerns and the need for schools to be places that encourage students to be an active part of the change required to address our physical world.

\section{Implication of purposes}

This section has identified the need for strong principal leadership identity, which often influences the trajectory of the wider school culture. To manage the hopes and ideas of the whole learning community, a principal needs a willingness to establish a vision that blends personal identities, cultures and context together. Broader issues related to the economy, social justice and ecological wellbeing, are further requirements, each as pressing as the other. It is the coming together of the varied purposes that ensure principal leadership practice is focused on holistic wellbeing.

\subsubsection{Leadership behaviour}

Leadership behaviour is the third theme evident in the research. Personal, relational, and collective behaviours are required of leaders and leadership. A leader needs to be adept at working both independently of others and interdependently with others to ensure change priorities are enacted.

\footnotetext{
10 Māori words for spiritual and holistic wellbeing.
} 
Personal

Personal behaviours exhibited by leaders encourage others to become a part of their work and are alternatively identified in the literature as traits (Zaccaro, 2007), skills (Robertson, 2016), dispositions (Claxton, Costa \& Kallick, 2016) or qualities (Sergiovanni, 2003). Often these are evidence of a leader's own personality and experiences (Ibara, 2010). The two examples listed here are not meant to be a distillation of behaviours but rather a representative sampling of those most relevant to this study and that give the greatest chance of establishing and maintaining connections among members of the schooling community. The personal behaviours of significance are emotional intelligence inclusive of social competence and selfawareness, and a sense of humour.

Emotionally intelligent (EI) leaders understand and manage their emotions (Goleman, Boyatzis, \& McKee, 2013). Also known as emotional literacy, (Claxton, 2005), EI is defined by Goleman and Senge (2014) as:

\footnotetext{
The capacity of individuals to recognise their own and others' emotions, to discriminate between different feelings and level them appropriately, to use emotional information to guide thinking and behaviour, and to manage and/or adjust emotions to adapt environments or achieve one's goal(s). (p. 248)
}

In a study on principal pre-service training, Govia (2017) recommends that papers on EI be factored into leadership development programmes, since it is an especially useful way to develop trust and mutual support. EI signals the ability of the leader to be attuned to self and others.

Self-awareness is the first important part of EI, and essential for leaders as they motivate, encourage and inspire others to work collaboratively. A qualitative case study by Gibson (2014) explored spirituality in principal leaders, and their influence on teachers in three public primary school contexts in NZ. This study advances a case for an external agent to help critique a principal's personal paradigm, to promote increased self-awareness and an understanding of why they think and act as they do. Gibson states that self-awareness contributes to the paradigm from which selfmotivation is implemented and therefore matters because it helps develop compassion. Leaders who are self-aware are adept at meeting not their own and others needs simultaneously. 
Social competency is the second most important aspect of EI. This requires an awareness of those around you and the ability to manage social situations capably, including power relations. In leaders, this requires what Garvey Berger (2012) refers to as "intelligently cultivating growth" (p. 42). She refers to three habits of mind that help cultivate wisdom. First, asking questions that are both harder to ask and answer. Barrett and Budge (2012) refer to questions that build leadership capacity, foster a safe and inclusive environment and focus on the learning of the system proper. Second, taking multiple perspectives with the aim of synthesising across differences and identifying more inclusive solutions. Thirdly, noticing patterns in your own thinking and in the organisation itself. Leaders who develop social competency in self and others, are cultivating organisational growth.

The use of humour is an important, yet under-researched attribute that positively influences school culture. Results of a study by Hurren (2006) support the idea that principal leaders who share humour have teachers with higher job satisfaction and there is a greater likelihood that the network remains intact in times of difficulty. Mascolo (2014) additionally suggests that shared humour generates higher perceived levels of effectiveness. Humour is one strategy for keeping members of the network connected during times of unexpected volatility.

\section{Relational}

The building of relationships is the most important group behaviour evidenced in the educational leadership literature. Quality relationships enhance a learning system through reciprocity. Surprisingly, Lowenhaupt (2014) argues that less attention has been directed to the role of conversation as a way of building connections and yet it is this form of communication that provides an effective first step for improving relations. Campbell and Nieuwerburgh (2017) additionally refer to a coaching model, which helps develop a simple method for examining reality, problem-solving, and setting goals. Such models contribute significantly to the success of relationships (Groysberg \& Slind, 2012). To explore and solve the unknown starts simply with a group's ability to converse effectively.

Wylie (2017) identifies practices that help focus principals on developing productive relationships in the $\mathrm{NZ}$ context, including: 
- developing 21 st century skills such as critical thinking

- drawing on difference as a resource

- $\quad$ supporting Māori student identities

- $\quad$ strengthening partnerships with parents and whānau

- developing teaching as inquiry

- $\quad$ ensuring that teachers get the time they need to undertake inquiry and collaborative work by reworking school days and allocations. (p. 9)

These practices are focused on drawing people and resources together and addressing aspects of power to increase the likelihood of the effective performance of not just the principal leader, but the collective.

\section{Collective}

A collective, cohesive display of behaviours improves the system's ability to selforganise and make substantive change. Leadership focused on collective behaviours includes: systems leadership (Senge, 2006), generative leadership (Groysberg \& Slind, 2012), or adaptive leadership (Heifetz et al., 2009). Bateson (2016) argues that leaders of these kinds are highly capable of coping with change naturally. This does not mean the system is left to evolve without direction but rather is steered or nudged by leaders towards a preferred trajectory. Three main collective leadership behaviours were evident in the literature.

The first was the ability of the leadership phenomenon to work with tension. As leadership practice becomes more complex, decisions become more difficult to make and leaders are frequently called upon to manage opposing pressures. The role of the leaders is to help create a balance between stability and flow and this is especially required in times of crisis and accountability (Shields, 2013). To achieve more balance, leaders may be required to influence ideas related to teaching, student achievement, and parental involvement (Elmore, 2000) or cease to continue with that which is not working (Parrett \& Budge, 2012) and requires leaders to be comfortable with uncertainty (Bateson,2016). Additionally, formal organisational structures such as those mentioned by Capra and Luisi (2016), including "formal policies and procedures" (p. 317), may help develop a feeling of security and reduce the stress on school staff. Shaked and Schechter (2013) suggest that leaders must learn to distinguish between important and not important issues for the greatest possibility of success and balance. The organisation learns to be comfortable with the unknown. 
The second collective leadership behaviour was the ability of those in the organisation to adapt. Adaptable leadership involves the aforementioned skills such as: establishing a clear and shared vision, engaging decision-makers at every level, empowering staff and students, and evolving the approach taken (Fullan, 2007). These skills are more and more vital in the modern world where leaders are expected to deal with complex issues (Hooper \& Bernhardt, 2016). Based on the principles of shared responsibility and continuous learning spoken of by Ackerman, Mette and Biddle (2018), adaptability brings innovative and strategic solutions to context. Adaptability is both an individual leader and collective leadership requirement.

The third adaptation that may be expected of leadership, is the dismantling of organisational structures. Fullan (2005) argues that schools have mostly inflexible organisational structures but that these nevertheless require 'unbundling' (cited in Hess \& Meeks, 2010). Parrett and Budge (2012) and Walker, Kutsyuruba and BishopYong (2011) see the creation of more flexible structures as a key leadership challenge and Goldstein, Hazy, and Lichtenstein (2010) and Fullan (2009) believe it is crucial if the school system is going to transform. There is, however, little written about how leaders assess their context and environment day to day and even situation by situation, to create conditions for success (Shoho, Barnett, \& Cyprès, 2010). The way leadership as a collective adapts and changes the current system it works within, requires greater investigation.

The third collective leadership consideration is an ability to overlap hierarchies. Such hierarchies aim to produce more frequent interactions in environments that are not necessarily known in advance. Leadership must pay attention to emerging conditions or level jumping (Gilbert, 2015). Level jumping is the ability to move across levels of an organisation to address vital unanswered questions (Davis \& Sumara, 2006). This principle is most evident in shared or distributed leadership (Shaked \& Schechter, 2013), where roles and responsibilities are more fluid, the mutual participation of learners encouraged, but the exact course to fulfil shared goals unknown. With leaders at many levels, and moving in many different directions, leadership behaviours can be expected to criss-cross the schooling context. 
Implications of behaviours

This section has shown that individual, relational, and collective leadership behaviours are important for safeguarding cohesion. Development of the various behaviours sees an ability of leaders to work independently of others and in relation to others. In turn, it is possible to build successful interdependent learning relationships so practice patterns are repeatable. A self-organising system requires a different set of behaviours to manage complex tensions, be aware of change, and be comfortable with uncertainty. Principal leaders model what is expected for the wider school system to allow for engagement at all levels.

\subsubsection{Leadership effectiveness}

Leadership effectiveness is the fourth theme evident in the research. Best practice, next practice and systemic progress are ideas in the literature that explore how the knowing, showing and/or growing of effective practice is conceived. Although diversity of opinions continues to emerge, it is clear effective practice requires a leader who is willing to improve the potential of both self and others.

\section{Summative progress measures}

The most commonly used summative measure of leadership success is student outcomes (Coelli \& Green, 2012; Day, Gu \& Sammons 2016; Grootenboer \& Hardy, 2017; Robinson et al., 2009). Achieving positive student outcomes requires the use of good quality leadership practices. The term 'best practice' is widespread in policy documents to indicate how effective leaders support positive student outcomes, and a direct correlation is assumed. Robinson et al., (2009), in the Leadership BES, identified leadership dimensions that directly impacted student outcomes or where "leaders interacted with others" (p. 36) and included: establishing goals and expectations; resourcing strategically; planning, coordinating, and evaluating teaching and curriculum; promoting and participating in teacher learning and development; and ensuring an orderly and supportive environment, the latter having minimal effects on learning. The dimensions from indirect evidence or "where leaders changed the conditions in which people work" (Robinson et al., 2009, p. 36) included: creating educationally powerful connections; engaging in constructive problem talk; and selecting, developing and using smart tools. The main finding was that when school leaders participate in teacher professional learning, outcomes improve. However, findings by the Professional Development Advisory Group (MOE, 2014) maintain that 
while sharing learning was of utmost importance, current PLD initiatives in NZ are not always well aligned with school/ kura processes or learning needs.

A point of view common in leadership literature is that practices, such as those described above, ignore socio-cultural and contextual features privileging the status quo knowledge about what works (Laddson- Billings, 2014). This focus on 'knowing' has seen leadership become more of a technical task, rather than a human task, in that there is too great a focus on accountability with "virtually no relationship to knowledge and practice improvement" (Elmore, 2000, p. 113). Dehumanising leadership is referred to by Wright (2011) as the "bastardising" of leadership (p. 345). Fullan (2011), Gunter (2001) and Thrupp and Willmott (2003) argue that the ever-increasing need to meet accountability pressures and regulate education with the realities of practice is counterproductive to effectiveness. This pre-determined way of being is not compatible with complex thinking (Davis, Sumara, \& Luce-Kapler, 2015). Hargreaves and Fullan (1998) concur, arguing that you can never know exactly how to advance and instead must learn through doing. Pre-determined practices cannot be expected to address all that matters.

\section{Formative progress measures}

A method of formative assessment, next practice is focused on creating practical improvements. This coming together of reflection and adaptation, is what Wright (2011) refers to 'next' practice. A willingness of leaders to work through the unknown but alongside others, and show a mindfulness of the complexities, growth and development needs of those involved. Wright (2011) outlines three main aspects that allow next practice to be more viable: phronesis, which is the ability to judge based on practical knowledge and helps with the ability to respond to difficulty; contextual intelligence, or the ability to draw on prior learning and apply the learning to the current context, working towards a preferred future; and lastly, the ability to work with the unknown. These disclose a type of contextual intelligence (Kutz, 2016). Leadership emerges into a shared learning opportunity that is process driven and shows the ability of leaders to critically reflect.

\section{Systemic progress}

Building on the idea of next practice is that which requires a tighter coupling of leadership and learning and is referred to by Raelin (2016) as Leadership as Practice 
(LAP). LAP is a process-oriented theory that prioritises emergence and nonlinearity ${ }^{11}$ over control and logic, and where "there is an immersion into practices that are embedded within social realities and between people, objects and their institutions" (Raelin, 2016, p. 7). Leadership activity therefore emphasises the next practice spoken of earlier. Some LAP activities referred to by Raelin (2016) are the:

1. Constructing and reconstructing social order through everyday interactions and issues for consideration

2. Scanning to identify resources, information or technology that may positively contribute to programmes

3. Signalling to others the need for their contribution to that which already exists

4. Weaving interactions across new and existing networks using trust and shared meaning

5. Stabilising the system by offering feedback to evaluate effectiveness, create structural and behavioural changes and learn

6. Inviting those who have held back to participate

7. Unleashing - making sure that everyone who wishes to, has the chance to participate

8. Reflecting on past current and future experiences. (pp. 6-7)

Participation in such activities ensures engagement of the learning community (Leithwood, Harris, \& Hopkins, 2008; Spillane, Halverson, \& Diamond, 2001) but also acknowledges the ongoing need for both theory and reality to find that which is effective within and across the system.

Leadership characteristics that support systemic progress, are more specifically referred to in the literature. In NZ, Rangitiratanga (MOE, 2010) describes this as leadership that takes on the role of both teacher and learner/He Kaiako-Ako. More recently, Fullan (2018)) portrays $21^{\text {st }}$ century leadership as: dependent on real world requirements not limited to numeracy and literacy; leadership that engages as many as possible; and leadership that works outward into communities and upwards to challenge policies and societal dynamics. Additionally, Parrett and Budge (2012) refer to four specific leadership leverage points in support of systemic change: using data to

inform actions, resource distribution and management, and optimising time. Systemic leverage therefore has a learning focus, considers context and focuses on areas of leverage.

11 CAS refers to nonlinearity as individual and/or collective behaviours by actors who are dynamic and their consequence unpredictable. Small actions can cause completely unrelated large scale changes. 
Raelin (2016) and Fullan (2018) focus on leadership practice for systemic progress that grows engagement across schools and from within the wider community inclusive of the development of organisational change and policy and practice initiatives.

\section{Implications of effectiveness}

This section has shown that leadership in the past has been emphasised as effective when student outcomes were strong and best practice was in evidence. There is, however, growing attention on formative and systemic performance considerations where the focus is instead on the learning occurring between: the individual leader and the learning collective; summative and formative measures; and theoretical ideas and practice. Principal leaders are expected to transform the achievement gap within the bounds of accountability and expectation.

\subsubsection{Leadership in the LSE context}

Intrinsic to leadership, is the environment within which leaders work. The literature on social justice ${ }^{12}$, transformative ${ }^{13}$, inclusive ${ }^{14}$ and bridge leadership ${ }^{15}$ emphasises specific considerations for a leader working in the LSE context. In this section, the implications for leadership in the LSE context of each of the themes identified will be discussed.

\section{Positioning}

The challenging of the status quo and improving community-school partnerships help leaders to address power differentials in the LSE school setting. This context requires a leader who explicitly confronts norms. Paul and Elder (2013) state that where norms and assumptions go unquestioned, it is the school leader who is best placed to challenge others to reconceptualise beliefs and meanings about their experiences. Normore and Brooks (2017) attest to principals who are "prepared to challenge top-

12 Social justice leadership (Bishop, 2011) emphasises the belief that all students can and will reach their potential, and that schools organise equitable learning possibilities for all students.

13 Shields (2013) refers to transformative leadership as that which "takes account of material realities, disparities, and unfulfilled promises of the world in which our students live, and of working to ensure more equitable and inclusive opportunities for all" (p. 5).

14 Inclusive leadership (Mac Ruairc, Ottesen \& Precey, 2013) is synonymous with social justice and is concerned with inclusive leadership processes and outcomes for all.

15 Bridge leadership (Tooms \& Boske, 2010) connects leadership to social justice for schooling improvement

All four of the above leadership theories are also detailed in Appendix B. 
down processes, and in doing so, address oppression.” (p. 289). Shields (2013) refers to transformative leadership as a way to achieve this, a theory of leadership that accordingly starts with "taking account of material realities, disparities, and unfulfilled promises of the world in which our students live." (p. 5). Addressing the status quo helps to make schools a place where the familiar is made strange.

The LSE context requires a leader who is willing to challenge expectations about reasons for underachievement and low expectations (Shields, 2013). Agirdag, Houtte, Avermaet (2012), found that the single most important factor influencing the academic success of minority students was a leader's explicit challenge to, and rejection of deficit ideas to explain away student underachievement. Indeed, a transformative leader was also one that would counter negative beliefs and empower staff and students to: influence the conditions that impact the quality of curriculum and instruction delivered; target shifting aspects of the change process; encourage continuous learning among staff and allows for sharing throughout the school and community so that broader organisational goals are achieved (Hallinger, 2003). Thrupp (2017) identifies that in NZ ignoring the attitudes and power relations that maintain deficit thinking has proven ineffective and so far, has invalidated any attempts at pedagogical innovation. Deficit theorising is detrimental to the effectiveness of practice and by default, achievement.

Principal leaders in LSE schools (Stroh, 2015) are well positioned to support their communities (Parrett \& budge, 2012). Reed and Swaminathan (2014) advocate working from within the communities themselves, to remain in touch with what is needed. Empirical studies suggest that where the leader is strongly linked to place more community influence is possible (Wilson et al., 2017). Tubin (2017) concurs hypothesising that, "when a principal with habitus already aspires to a leadership position and believes in the dominant values of that place, it is easier to gain followers' approval.” (p. 802). Where others in the learning community might be less able, leaders are capable at both supporting and positively influence the collective.

\section{Purpose}

LSE leaders create a broader purpose to their work by showing a willingness to cultivate social change. Damaging views of schools and LSE communities means leaders must be willing to position themselves as community advocates (Duignan, 
2012; Tooms \& Boske, 2010; Stroh, 2015). Indeed, LSE principal networks are seen to be "spending time trying to compensate for social disadvantage by replicating the services middle class children already get in their homes, at school" (Carpenter \& Osborne, 2014, p. 29). Currently, it is posited that leading for social change requires leaders to lead "against the grain" and this will guarantee a greater balance of social entitlements are met (Shields, 2013, p. 2). In fact, Stroh (2015) postulates that the most effective way to create social change is for school leaders to work to ensure that expectations for schools are framed within the broader context of social investment. It is a consideration that social change in school does not occur in isolation, and its success often lies in the wider system.

Leadership in an LSE context sees leaders purposefully work to improve home-school conditions. Duncan and Murnane (2011) and Schafermeyer (2017) agree that the influences of families/whānau on the school community are key levers for high quality student outcomes, suggesting efforts are worthwhile. In NZ, Macfarlane and Derby (2018) promote a similar idea - that of a cultural advocate - a leader seen to develop community opportunities, especially for aiga/whānau (Mcfarlane \& Derby, 2018). Wenmoth (2014) states, however, that the pastoral care of students and their families has been forgotten in NZ as leaders instead work to address management considerations (Lupton, 2005; McIntosh \& Rima, 2007). An alternative point of view suggests this is not necessarily problematic. According to McNae et al. (2017), when leaders focus on issues related to aiga/whānau engagement, their attention is all too often drawn away from what communities ought to be engaged in, and that is developing and guiding the curriculum. Involving families in school life is mostly unsuccessful. Referred to by Snyder (2013) as systemic lock-in, Duncan and Murnane (2011) suggest that when there is a lack of improvement with home-school relations in LSE-type settings, it is likely to be because policymakers over-estimate a school's ability to alter family practices in ways that will reduce the achievement gap, and programmes outside of schools are more likely to be useful. Of interest is the role digital technologies have played in the way schools and families communicate, learn and solve problems together (Kemp, 2015). Bolstad (2017) refers to increased connectedness through digital opportunities with families in some schools. Synder (2013) advocates that this may be an example of technological breakthroughs disrupting a systemic lock-in. It appears the relationship between LSE leaders and their families requires ongoing consideration. 


\section{Behaviours}

Modelling moral passion and encouraging a positive school culture and climate are behaviours that encourage engagement across the LSE context.

Leaders in LSE settings show moral passion. This is sometimes referred to as moral literacy (Bezzina, 2013), or in NZ, manaakitanga (Bishop, Ladwig, \& Berryman, 2014). Well documented in the literature, and increasingly required in the $21^{\text {st }}$ century, is a LSE principal leader's willingness to position themselves ethically or for common good (Enomoto, Kramer, \& Starratt, 2007; Kramer, Enomoto, \& Enomoto, 2014; Parrett \& Budge, 2012; Shields, 2013). Notman (2017) suggests the complex phenomenon of leadership, especially for those leaders working in high-needs schooling contexts, is deeply moral. A threat to being moral is what Stewart (2006) refers to as a personal blind spot. For example, Stewart states that leaders may define their success by their achievement and possessions rather than their moral character. This is exacerbated by a system that does not reward moral courage. Recognised in the literature more recently is international research in 14 countries, implemented over the last 16 years for the International Successful School Principalship Project (ISSPP). This research showed that when principals are effective, they can challenge boundaries for moral good rather than just meet compliance and policy directives. This is a promising trajectory. Moral leadership is a well-documented consideration for leaders in the LSE context as a way of balancing accountability pressures with what is right.

Effective leadership in LSE contexts works hard to encourage behaviours in others that support a positive school culture as a vehicle for working together to exemplify the beliefs, values and assumptions they share (Deal \& Peterson, 2016). One way leaders achieve this is to position diversity positively (Enomoto et al., 2007; Kramer et al., 2014) so students do not have to wrestle with feelings of having to choose between their heritage and culture or assimilate to get ahead (Mulcahy, 2013). Paul and Elder (2013) urge school leaders to reflect more deeply on the issues of diversity and multiculturalism. Marion and Uhl-Bien (2001) offer a framework for supporting leader/s to create the conditions for generating change while also ensuring positivity and wellbeing. They suggest introducing and promoting new ideas that bind people together and encouraging systemic thinking so there is awareness of the dynamics taking place. 
Leadership in the LSE context works towards actions that encourage a safe school climate (Cotton, 2003; Jacobson, Brooks, Giles, Johnson, \& Ylimaki, 2007; Martin, 2010). In LSE community settings, where students are sometimes less safe, and environments more volatile, clear processes for addressing inappropriate behaviours (Parrett \& Budge, 2012), and a visibly present leader (Robinson et al., 2009) are especially important components that promote a positive climate (Cherkowski \& Ragoonaden, 2016). Blackwell (cited in Gordon, Jean-Louis \& Obiora, 2018) suggests that a safe learning environment, with consideration for aspects of the wider community, is fundamental to promoting and improving student learning and includes consideration for the presence of bullying (Craig, Pepler, \& Blais, 2007), which can affect students' academic and social wellbeing (Cherkowski \& Ragoonaden, 2016). Duncan and Murnane (2011) have reported that disadvantaged students who are academically more resilient, are likely to experience a good disciplinary climate compared to students at schools with more disruptive environments. A shared vision inclusive of a safe environment creates wellbeing of individuals and the collective culture.

\section{Effectiveness}

Focusing on ELL learner needs and having the right tools available, all help leadership to be able to effectively regenerate the system and be creative. The LSE context requires leadership that measures its effectiveness by how well the needs of the ELL learner are addressed. In a recent study, Moshayedi (2018) found that instructional strategies targeting English language acquisition both in tandem, and in addition to core subject-matter, were essential to improving learning outcomes in LSE schooling contexts. In NZ, ELL numbers continue to grow and there is a strong focus on developing the capacity to support ELL teaching and learning (McGee et al., 2014). Competency in a second language is recognised as an indicator of further success, which is perhaps under-recognised.

Leadership in the LSE context is effective when learning tools are made available. Wylie (cited in Rashbrooke, 2013) and Day et al. (2016) state that schools located in LSE areas have difficulty drawing on a full range of knowledge, skills and opportunities to encourage students. Adams (2015) advocate for principals leveraging off different pathways for connecting students' growth and development opportunities. Ovens and Godber (2012) refer specifically to affordance networking as one way of managing this. This form of networking emphasises new knowledge from beyond the 
immediate school community. Ainscow (2015) alternatively refer to this as a redistribution of resources that already exist and Parrett and Budge (2012) draw on the idea of community-based service and learning where students participate in community outreach. Leaders in LSE schools need to be effective at networking for resourcing and finding alternative learning opportunities for students (Portin, 2000) as well as reciprocating and contributing back to the community. Parrett and Budge (2012) argue also for the need to be aware of the hidden costs of schooling including "athletics, band, choir, various clubs, as well as materials and supplies related to homework assignments, athletic uniforms, yearbooks, school pictures, social activities and school supplies.” (p.75). As such, Shields (2013) proposes that schools should no longer be silos apart from the community and that instead, private sectors, civil society organisations, and governmental agencies together play a role in reducing poverty alleviation (McKague, Wheeler, \& Karnani, 2015). Baker (2018) debates that while money alone is not the answer, increased financial input is necessary for the improvement of educational outcomes. The allocation of funds is positively associated with student success, especially when used strategically (Giles \& Palmer, 2015). Achieving student success is more challenging without the right level of resourcing.

\section{Implications of context}

The formal structures available to leaders in the LSE context, such as staffing and finances are limited, while informal structures such as the constant need to challenge the status quo and deficit mentalities add a further strain. Regardless, the principal leader's role is to guarantee all environmental needs are met so students and professionals can learn. Additionally, adjustments need to be made about what success can look like in the LSE context and what is possible with the resources available. Principal leaders are heavily relied upon as change agents in the LSE context. Aristotle's concept of practical wisdom or the mean of virtue (cited in Dunne, 1993) would be useful when working on the three behaviours described above. Practical wisdom not only requires individuals to apply learning to context, but require actions taken, to be at the right time, for the right reason. Practical wisdom also requires personal theories, and interpretations of contextual factors viewed through the lens of personally-held morals (Dunne, 1993). The melding of the LSE context requires the melding together of a leader's beliefs, leadership theory and context and may be a useful way to address resource inequalities. 


\subsection{The merging of leadership themes}

Leadership positioning, purpose, behaviours, and effectiveness have all been discussed as separate themes in the literature. The links between aspects of each theme helps identify desirable patterns of leadership practice and the implication on the leaders of tomorrow. Broad pedagogical implications are considered.

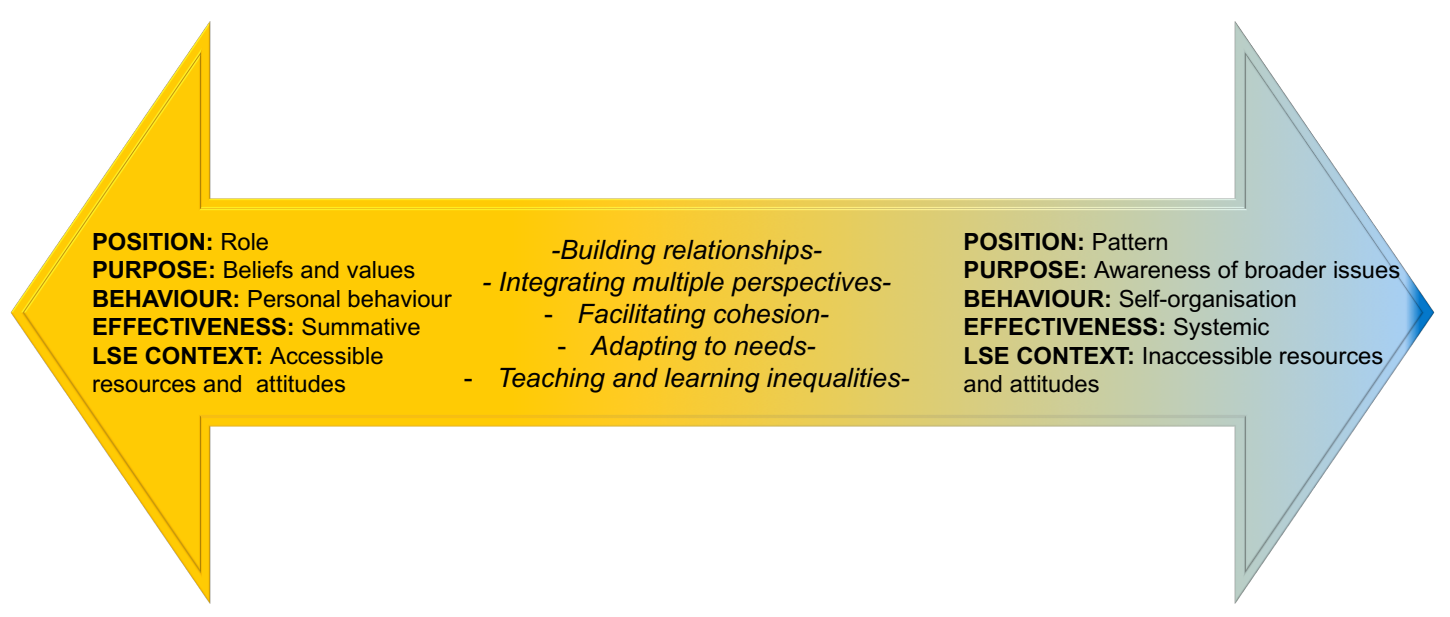

Figure 1: A continuum of themes and patterns in practice

Five patterns of practice appear to have emerged from the literature and these can be seen in Figure 1 above. The first pattern of practice is 'building relationships'. The most obvious leadership theory to explore interactions and a leader's ability to create positive relationships (Bryk \& Schneider, 2003) is relational leadership theory (Agosoto \& Roland, 2018; Giles \& Palmer, 2015; Bryk et al.2015). Eppinga, Salina, Girtz and Martinez (2018) advocate that leaders make seven 'shifts' for relational improvement. These are: developing a common language, reassessing privilege, having one-to-one conversations, including support staff, having collaborative inquiry teams, building support systems and giving permission to innovate. It appears therefore, that $21^{\text {st }}$ century leaders must consequently encourage strong, trusting and reciprocal relationships.

The second pattern of practice is that of 'integrating multiple perspectives'. A pertinent leadership theory that explores perspective is culturally responsive leadership theory (Baldwin, 2015; Hoque, 2018). Culturally responsive leaders support pedagogical and social inclusion of students values, histories and cultural knowledge (Johnson, 
2014; Santamaría \& Santamaría, 2016), generate adaptive school structures to empower students and families who are under-served (Madsen \& Mobekela, 2005; Santamaría \& Santamaría, 2016; Villegas \& Lucas, 2002), and work towards developing critical consciousness and pedagogy among students and faculty (Johnson, 2014; Santamaría \& Santamaría, 2016; Villegas \& Lucas, 2002). Therefore, tomorrow's leaders must be prepared to include various ways of being into 'the way we do things around here' (Deal \& Kennedy, 1983).

The third pattern of practice is that of 'facilitating cohesion'. Cohesion is the art of forming a united whole. One main leadership theory in support of this notion is that of systemic leadership (Senge, 2006). Facing mounting complexity and diversity, school leaders need to consistently focus on the use of a broader lens to address issues or concerns faced by the collective and reduce the use of silos and fragmentation (Heck $\&$ Weiss, 2005). Senge (2006) refers to a systems leader as one who has a deep intention to engage in collective reflection to transform practice. According to the James Irving Foundation (2017), this is often by way of a novel or unexpected approach, so that the difficulties experienced by the larger system are better understood and a wider range of change levers become apparent. Consequently, leaders need to be robust systems thinkers that encourage interconnected thoughts and actions.

The fourth pattern of practice is that of 'increasing effectiveness'. One leadership theory in support of this notion is motion leadership (Fullan, 2009). Concerned with moving from less effective to more effective results, motion leadership helps leaders develop an understanding about the nature of change, see learning as both an individual and organisational task, and develop the capacity of others. Fullan (2009) advocates that motion leadership sets an expectation of constant movement for improvement from individuals and the organisation whole. Forthcoming leaders that are adaptable to the change process, can develop a much wider sphere of influence over the learning organisation.

An unexpected, fifth pattern of practice is that of 'teaching and learning inequalities' and emerged from the need to address the LSE context more fully across all four prior themes. Considerations included: how power differentials may help or hinder positive relationship growth; the need to challenge status quo and cultivate social change; the preparedness of a positive and safe school culture; the need to respond to context; and 
an awareness of how economic status affects learning. A pertinent leadership theory that explores such considerations is that of transformative leadership (Shields, 2013). Shields (2013) suggests transformative leadership starts from the position of social justice, which requires leaders to learn about how the distribution of wealth, opportunities and privilege affect minority groups. For school leaders to encourage a minority perspective they must work to eliminate teacher deficit thinking, have difficult conversations and model preferred practice (Shields, 2013). Shields also suggests giving permission for teachers to do the same in classrooms. Therefore, upcoming leaders that understand the impact of the LSE context on learning and learning resources and attitudes are likely to more proficiently address teaching and learning inequalities.

The five practice patterns provide some examples of pedagogical implications which are likely to improve both engagement and learning, specific to students in the LSE context, and includes a need for leaders to:

- $\quad$ support the development of a connected learning network

- develop an inclusive yet critical learning culture

- develop a positively interdependent learning network

- advance change for improvement

- emerge a dual focus on academic and social needs.

These practices identify the need for a tighter coupling of leadership and learning, leadership that helps create fluidity between those learning and those leading. It is this idea, that moves leadership practice into a complex realm.

\subsection{Defining educational success}

In this part of the literature review I turn my attention to the original motivation for this study, improving achievement levels for students over-represented in the tail of underachievement. I explore notions of success to understand better how the current trajectory is constructed and why success has not been occurring. This section concludes by exploring implications for principal leadership. 
What we measure highly influences how we define success. Western nations generally measure success in the form of test score outcomes. Nationally-normed assessments help teachers and leaders to make comparisons of the academic performances of individuals. Tests, however, are limiting as they give leaders no way of knowing how much latent talent is going unrecognised, do not in fact demonstrate progress, place no value on human difference (Wan, 2008), and ignore the simple 'feeling' of success (Manu'atu, 2000) that may manifest. Test measures are incapable of showing incremental and immeasurable changes (Snyder, 2013). Current measures of success perpetuate a narrow agenda of achievement.

Literature acknowledges success as a journey as well as an outcome. Wheatley and Frieze (2011) suggest developing more germane measures of progress to make achievements visible, but also regularly reflect on the learning occurring. Bryk, Gomez, Grunow and LeMahieu (2015) refer to this as the measurement of intermediate outcomes to provide tighter measures on how students are moving forward. These allow for regular reflection on how learning is tracking and how far learners have journeyed (Wheatley \& Frieze, 2011). Assessment measures that show the changes occurring offer a way to re-focus on the incremental and less concrete successes experienced.

A broadening of the notion of educational success has subsequently been the focus of several international studies. A longitudinal project on student success in Canada (People for Education, 2013) looked to expand the indicators used to measure progress and which included academic, physical and mental health, creativity and innovation, citizenship and democracy, and school climate. Madden (2014) argues more specifically in NZ for educational success which includes the improvement of social and emotional skills. This helps to foreground measurable outcomes which by themselves were considered by McKinley and Hoskins (2011) as too limiting to identify changes to educational achievement patterns of Māori and other minority groups in NZ. McKinley and Hoskins (2011) suggest widening educational success to include: problem-solving, resiliency, collaboration, resilience, and self-management since it is these kinds of skills, he suggests, that are portable and teachable across the system. This research demonstrates that what we measure is beginning to be challenged by what some are calling 'soft skills' (Claxton, Costa, \& Kallick, 2016; 
Zepke \& Leach, 2010). Success may therefore include that which is difficult or unable to be measured.

What students in NZ believe educational success to be is also of relevance to arguments about how success should or could be defined. A recent report about progress and achievement from the point of view of students was issued by the NZ Children's Commissioner (2018). In it, students spoke of a broad view of success which included attending school and trying to improve. The seven key findings of the report highlighted that students wanted: their strengths to be recognised; help in understanding their goals; relationships to be central to attaining achievement; to make their whānau proud; a physical space that positively impacted on their learning; to work more outside of the classroom; and for bullying to be controlled. These aspects are more in line with the evolving ideas of success mentioned above.

Reasons for a lack of success are widely explored in the literature. Ladson-Billings (2006) and Shields (2013) concur that a lack of success indicates a systemic inequity whereby resources required to achieve for cohorts of greater diversity are unavailable. Ladson-Billings penned the term "education debt" (p. 8) to illustrate this point. Education debt describes inequalities existing within education practices and systems such as the lack of a powerful curriculum (Parrett \& Budge, 2012) and a narrow resourcing agenda (Reyes, Scribner, \& Scribner, 1999; Snyder, 2013) inclusive of a digital divide (Ragnedda \& Muschert, 2013). Tooms and Boske (2010) acknowledge individual learning differences and suggest care is needed to select the right tools and resources. It is therefore not surprising that children of LSE status are not achieving, given they are less likely to have had the opportunities for quality early childhood learning opportunities, well-resourced schools, quality teachers, and communities with abundant resources to contribute.

Tollefson and Magdaleno (2016) focus on the need for teachers and leaders to develop their perceptions of what success looks like. To close what they call 'the acknowledgement gap', Tollefson \& Magdaleno (2016) suggest: discussing examples of the gap with colleagues; studying alienating school factors; cultivating awareness of bias; valuing teachers who are culturally responsive; welcoming and communicating equally with parents; and cultivating hope, agency and a culture of praxis. In a study on problematising the achievement gap in mathematics research, 
Gutiérrez (2008) proposed a focus on gains and doing less of documenting the achievement gap; what is causing it; and putting a focus on single variables. Alternatively, Gutiérrez (2008) proposed the refocusing of research on effective teaching for ELL and working class students that includes richer descriptions of those environments (p. 362). The current challenge is to ensure that views of excellence in academic achievement are constructed, not only by objective measures and measurable outcomes, but in holistic ways that consider the health and wellbeing (Parrett \& Budge, 2012) of more diverse individuals in society.

\subsubsection{Implications for principal leadership}

Principal leaders can support a change in the success culture. Botton (2008) and Ladd (2012) state that schools should be accountable for the internal policies and practices that help to produce a broader set of holistic educational successes than can be measured by test scores alone. Ensuring specific familiarity with legal and ethical requirements ensures all students are adequately prepared "not simply to pass tests but to take their place as informed, caring and engaged citizens," (Reeves, 2008, p. 5). Principal leaders are involved in systemic change that may help to develop civic-minded citizens.

The principal is charged with identifying what notions of success can be currently resourced in their context. Principals work to provide more of what is needed as well as creating structures and processes that take into greater consideration the holistic nature of success, including the spiritual and moral development of students (Duignan, 2012). A wide variety of resources are required to ensure wellbeing, a prerequisite for success.

Principals should also consider the value of local experiences. Blumen (2016), in her synthesis on empirical studies that looked at high achieving but deprived young people in South America, concluded that to face the challenges of a changing and complex world, high-achieving deprived youth need to develop expertise in the conditions they face within and beyond their local contexts and assert these perspectives widely. Solving local problems acts as a vehicle for helping students bring local successes to a global audience.

Educational leaders must acknowledge the cultural gaps between themselves and the communities they serve. Tollefson and Magdaleno (2016) posited the idea that a lack 
of success indicates a cultural gap, suggesting a greater need for leaders to understand and value the role culture plays in facilitating or hindering academic success. Tollefson and Magdaleno in their study on gap-gazing in primary mathematics education, identified four dimensions for achieving success: access to resources; improved standardised test scores and participation rates; maintaining cultural, linguistic and familial identity; and power or agency to effect change in school or society (p. 360). Their study suggests moving beyond access and achievement (which are more often addressed) to identity and power. Intervening to change reality requires critically conscious leadership behaviours.

\subsection{Gaps in the literature}

The literature review identified significant gaps. Five gaps in the literature summarised here illustrate an understanding of the challenges principal leadership practice currently faces.

Firstly, the principal holds various positions across classroom, school and community contexts, yet exploring principal leadership as both an individual and a collective pursuit is, according to Tollefson and Magdaleno (2016), relatively rare. While possible patterns of practice were identified in the literature, studies pertaining to principal leadership patterns of practice are rarely found in empirical research, even though Lambert (2002a) cites patterns of relationships as the basis of human growth and development. It would seem prudent that any new educational research should attempt to explore principal leadership as a focus on the individual, the collective, and how wider patterns of practice manifest in day-to-day working lives.

Secondly, there are various purposes behind how and why principal leaders act as they do and these range from personally-held beliefs and values to broader considerations. There is research available in educational settings on the beliefs and values of various practioners (Day et al., 2011; Notman, 2014), yet a gap remains in research about primary principals' values-based leadership practice in the NZ primary school setting. We are yet to learn how a principal's own beliefs and values influence the focus of outcomes in a NZ LSE primary school setting. 
Thirdly, the review revealed the strong influence that context has on leadership practice (Thrupp, 2015) and yet a rich description of the interaction between LSE context and principal leadership is a neglected focus of educational leadership literature in NZ. We know that schools are becoming more diverse and economic status is impacting on the learning context for the student community, but how the LSE context influences the engagement of principal leaders and how leaders are affected by the LSE context is yet to be researched to any great extent in New Zealand. It would seem important to understand the story of underachievement, to find out what expertise principals are developing to address the conditions they face. This research aims to bring the context into focus, not as an excuse for educational underachievement, but to broaden perceptions of problems and solutions.

Fourthly, leadership in the $21^{\text {st }}$ century requires an ability to work with uncertainty and constant change. Showing how the principal leader works with evolving dynamics and harnesses desired change is a valuable step in linking leadership and learning more closely together. Patterns of practice are rarely explored and there are many unanswered questions about how to do this. This research will be an attempt to address both these concerns. Organisations have always been complex but the ability to understand them as complex remains limited (Goldstein et al., 2010).

Lastly, educational research tends to reflect a narrow perspective on success. The notion is influenced by what is measurable and accepted as valued by the status quo and with little consideration for culture and the immeasurable. What local LSE communities perceive as success and what the principal leader is doing to widen the system's ability to improve value-added outcomes for students in the LSE context is the last consideration of this research.

Together these five gaps in the literature inform this research and provide a clear research premise (see Table 2 below). The research will explore how principal leaders practise leadership in an LSE context for educational success. 
Table 2: Linking the literature review to the research questions/s

\begin{tabular}{|l|l|}
\hline \multicolumn{1}{|c|}{ Key aspects of literature review } & \multicolumn{1}{c|}{ Research question foci } \\
\hline $\begin{array}{l}\text { A lack of investigation into patterns of } \\
\text { principal leadership practice }\end{array}$ & Patterns of practice \\
\hline $\begin{array}{l}\text { A lack of attention to context and its } \\
\text { influence on principal practice }\end{array}$ & Contextual considerations \\
\hline $\begin{array}{l}\text { A gap in our understanding about primary } \\
\text { principals' values-based practice in NZ }\end{array}$ & Influence of beliefs, values and ideas \\
\hline $\begin{array}{l}\text { Identification of a narrow definition of } \\
\text { educational success and an even more } \\
\text { narrow way of measuring success }\end{array}$ & Perceptions of educational success \\
\hline
\end{tabular}

\subsection{Chapter summary}

The literature review has provided an overview of the current literature with relevance to leadership practice in LSE school settings in NZ for improved student achievement. Educational leadership was contextualised and appropriate empirical research included to indicate a shifting trend towards a complex phenomenon considerate of context, theory, practice, and people. The research review focused on five themes that illustrate what the challenges of leadership practice in a LSE setting are and the trajectory that the theory is taking from simplistic to more complex notions of leadership. These themes were: building relationships, integrating multiple perspectives, facilitating cohesion, adapting to need, and showing an awareness of teaching and learning inequalities. Considering the findings, there is a clear need to contextualise the problem of underachievement, as well as broaden the research methods used to capture the complexity of the impact of principal leadership practice. An alternative perspective will offer a renewed focus on the interrelationship between leadership and learning as a lever for transformational change. 


\section{CHAPTER 3 \\ The theoretical framework}

"There is nothing so practical as a good theory" Kurt Lewin

\subsection{Chapter overview}

This chapter explains the quest for the ideal theoretical framing for the study. Picking up from where I left off with my personal theory, and the subsequent awareness of social inequity, I start by explaining the purpose behind using Bronfenbrenner's (1979) ecological systems theory to help depict the leadership context. Next, complex systems theory (Byrne, 1998; Cilliers, 2002; Lemke \& Sabelli, 2008; Mason, 2008; Senge, 2006) offered a way of seeing leadership as a set of individual and collective interactions that make up a natural eco-social change process. Finally, a holistic theory by Capra (1997) introduces these ideas into this research in a novel way. The iterations assemble a framework that emerged to suit the study aim and objectives outlined in Chapter one.

\subsection{Building the framework}

The literature review in Chapter Two showed that principal educational leadership in the $21^{\text {st }}$ century: involves individual and collective behaviours as well as patterns of practice between people; is influenced by context; is an ever-evolving practice that works to address and harness uncertainty and change; and is a challenge to capture. Significant attention is given to the design of the theoretical framework so these aspects and their implications might be identifiable.

The metaphor of a camera's aperture ${ }^{16}$ helps to explain a growing sophistication of thinking that I encountered as the research progressed. With a wide aperture, the image background is indistinct, and the depth of field is shallow. When the gap narrows, both the background and foreground can be in focus at the same time.

Figure 2 shows three different apertures (seen in orange) used at various stages of the research. Rather than starting the research knowing I would need to adjust my

\footnotetext{
16 The aperture of a camera is the opening of a lens's diaphragm through which light passes.
} 
theoretical stance, it became apparent as the investigation proceeded that the appropriate aperture would emerge much like it would for a photographer experimenting to get the perfect action shot. Each time the opening was narrowed, the lens refocused, and light reapplied, the theories for use in this research became more and more apparent. Experimenting with theory eventually allowed for a more reflective analytical stance to be utilised in this thesis.

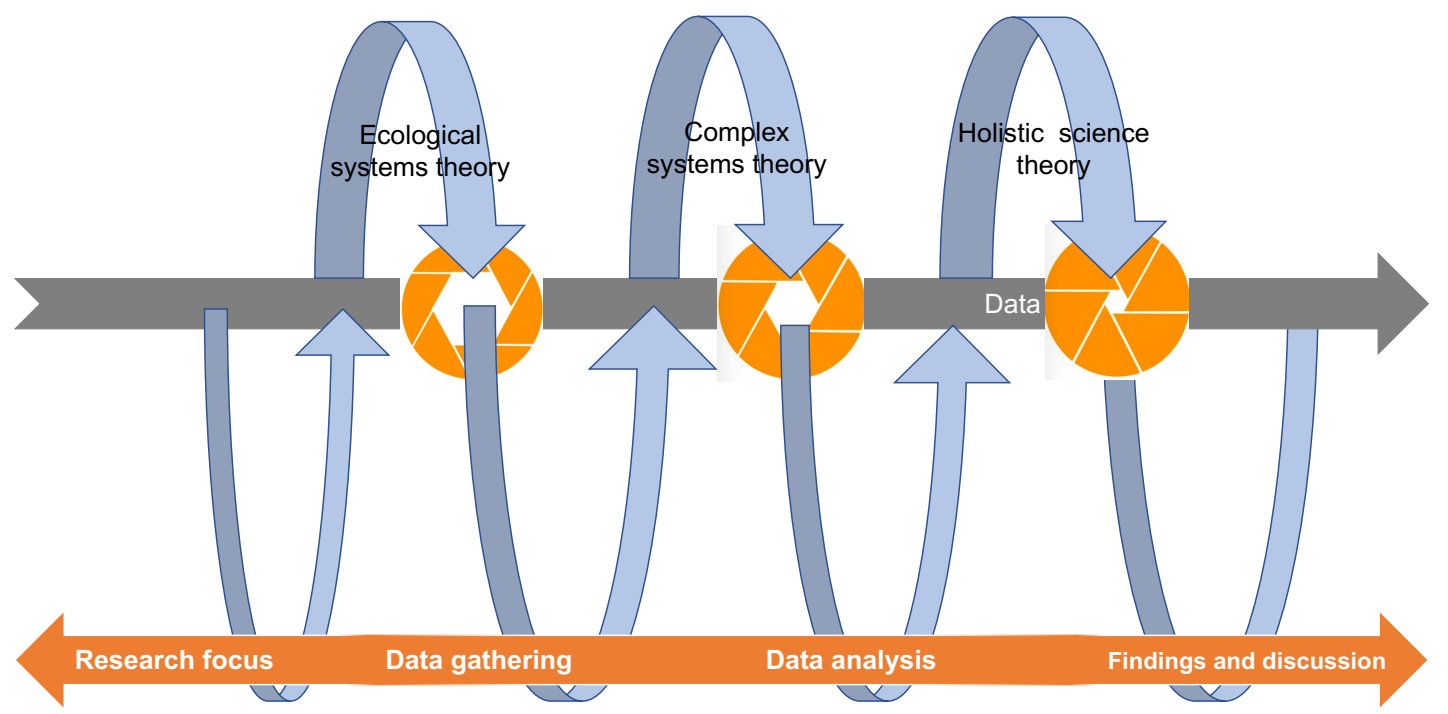

Figure 2: 'The Building of the theoretical framework.'

The discussion of my background, education, learning philosophies and professional experiences, and my awareness of social inequity in the introductory chapter gives some clues to my personal theory through which this research was approached. My heightened awareness of social inequity and growing knowledge from the literature of the likely impact a principal leader could have on improving achievement, reducing education debt, and improving social reform became my starting point for the research and a personal lens through which research actions and decisions are viewed.

\subsubsection{Ecological systems theory}

My training as a teacher and advisor in special education led me to understand the significance of context. This first, deliberate narrowing of the camera aperture signalled a focus on the context which was mindful of both people and environment.

Bronfenbrenner (1979) recently renamed bio-ecological systems theory (Naglieri \& Goldstein, 2011), indicating that the interaction between a child's biological features 
and context are both important considerations in their development. His original theory was introduced after World War One in the USA as a response to the limited capacity of research undertaken by developmental psychologists. Bronfenbrenner conceived the idea that to understand child human development, the entire system in which growth occurs also needed consideration. Bronfenbrenner offered it as a theory in the 1980s and continued to modify it until his death.

Named in the Bronfenbrenner model are five levels of relationships that form a child's developmental environment. These are arranged from closest to the individual to more distant, with influences both within and between people. The theory reflects the idea that multiple environmental systems influence human development. These systems are the chrono, micro, meso, exo and macro systems, with each having a continuing impact on a child's development (Bronfenbrenner, 1979). Applied to my study, the chronosystem identifies the events and transitions that transpire throughout the life of the individual principal, while the micro-system contains the structures, relationships and interactions they have within their immediate surrounding (Bronfenbrenner, 1979). The mesosystem connects two or more micro-systems, for example between the principal and a child's teacher. The exo-system defines the more extensive social system in which the individual does not directly function but within which the individual interacts (Berk, 2000). An example would be wider community organisations. Lastly, the macro system includes large-scale influences such as politics and national policies affecting schools but involving national customs and values. While the criticisms of such a model include that it does not always acknowledge that humans cross boundaries to develop complex identities (Durr \& Hill, 2006), it does recognise the importance of context.

Bronfenbrenner's model was demonstrated to be useful for improving student achievement (Anderson, Boyle, \& Deppeler, 2014) and in educational leadership for understanding how leaders and teachers practise for change and the community's response (Slomp, Mombourquette, \& Marynowski, 2018). The theory has been applied to public health research. For example, studies looked at student dropout rates (Tyler, 2011), mental health (Eriksson, Ghazinour and Hammarström, 2018) and building healthy lifestyles (Cala \& Soriano, 2014). These studies recognised barriers to creating better health in adolescents, made mental health policy recommendations and predicted dropout rate demographics. Recent research by Eriksson et al. (2018) found 
that when used in the public health arena, the model most often came from the theory's early inception and showed no evidence of exploring the interactions between systems. As a result, it had mainly been used as a narrative device rather than as a guide towards understanding the practices that occurred across an ecosystem.

In my research, the adaptation of Bronfenbrenner's model (Figure 3) proved advantageous as a data-gathering framework showing the multiple environmental systems influencing principal leadership.

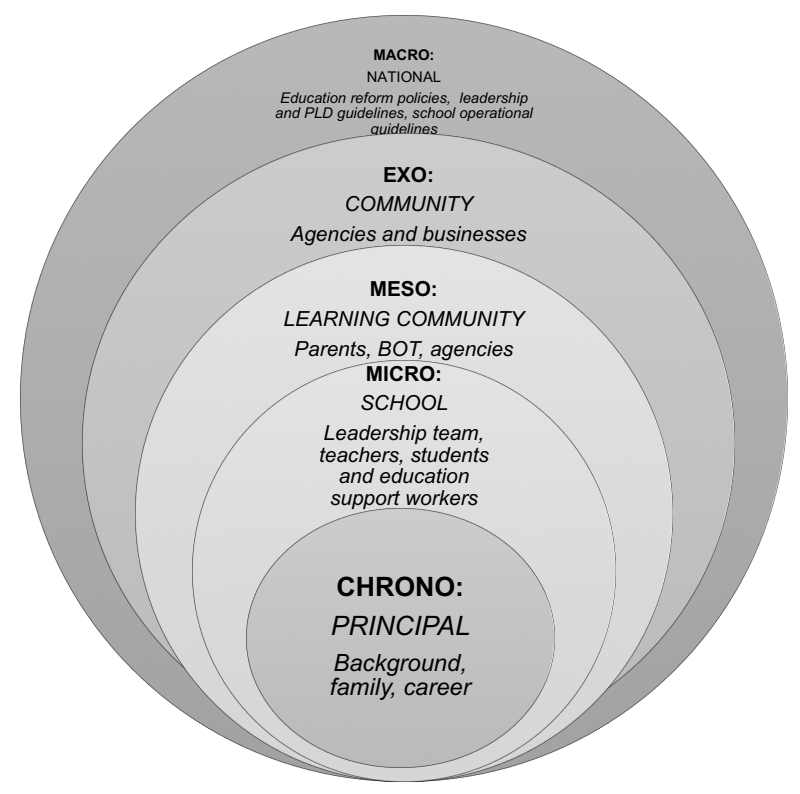

Figure 3: Adaptation of Bronfenbrenner's ecological systems framework model with the principal leader at the centre

The model outlines where data was gathered from and from whom. I still needed assistance to focus more fully on the interactions among the network members, the LSE school context, and any resulting changes. This focus would allow for the framework to be less fixed and more like an adaptive organism.

\subsubsection{Complexity Theory}

I considered a further theory that would give me a method of understanding principal leadership as less focused on reducing effectiveness down to a standard set of practices already evident in current literature, and more focused on the process and interaction for self-generation. I adjusted the aperture a second time to achieve this and focused on Complex Theory (Byrne, 1998; Mason, 2008). Complexity Theory focuses on connections, relationships, patterns and context. What becomes apparent when 
viewing my research through such a lens, is that various levels of scale are required if the study is to expose principal leadership practice more fully.

This theory exposed the complexity of principal leadership practice and supported a focus on the behaviours of the broader system (Morin \& Montuori, 2008), of which the principal leader is a part. Gilbert (2005) states:

\section{The IT revolution (among other things) has disrupted the modern world's tendency to see systems in terms of parts, or as distinct from each other. However, while the interconnectedness and interdependence of everything are now widely recognised, the challenge to "traditional" ways of thinking it offers is not. Complexity thinking has developed to fill this gap. (p. 9)}

Complexity theory innovates beyond the discipline-specific approach of experimental mathematics and thermodynamics (Byrne, 1998), or 'hard sciences' in which it originated. Often referred to as first-generation complexity, Alhadeff-Jones (2008) states this is where "information and communication theories, automata theories, and cybernetics and operation analysis - can be found at the root of this trend" (p. 65). Additionally, complexity theory extended into computer sciences, management, engineering and evolutionary biology and is the second generation of complexity which was spurred on by technological progress and large conglomerates (AlhadeffJones, 2008).

It is the third generation of complexity theory (Alhadeff-Jones, 2008) that I use for this research - Complex Adaptive Systems (CAS). This third generation of complexity theory emerged from the Santa Fe Institute in Mexico $^{17}$ through the merging of complexity theory with dynamic ecosystems theories. Systems theory, founded by Bertalanffy and described by Lewin (1976), shows a world made up of separate parts affecting one another, trying to adapt and change while holding on to identity. However, this third generation of complexity shows a more open boundary between the complexity and systems theories, with differences "dissolved somewhat, if not entirely" (Richardson, 2004, p. 75). CAS is a set of interactive and interdependent components that form a cohesive whole, mutually responding to environmental changes or changes between and within the interacting parts.

17 The Santa Fe Institute is a central research organisation devoted to the study of complex adaptive systems. 
Therefore, this research viewed the broad notion of context as a relationship between the components of people and place rather than as two separate entities showing people in place. According to Schein (2015), humanity and the environment are intrinsically linked and co-construct the ecosystem within which they live.

Some caveats apply to the use of complexity thinking (Davis et al., 2015). Morrison (2010) mentions issues such as, "A lack of a clear definition, and a theory that confuses explanation with the description, neglects ethical dimensions and risks invalidating reasonable expectations of accountability and responsibility" (p. 3). Regardless, Davis et al. (2015) continue to advocate that though complexity theory has its negatives, it still "offers the most cogent understanding of the nature of continuity and change" (p. 13). Consequently, I present not a definition, but the concepts of complexity in ecological, social and educational terms. In doing this, I address all the concerns listed here.

\section{Complexity theory and ecology}

Leadership as a living system is understood here in terms of an underlying processes. I use five concepts to describe Complex Systems Theory as a self-generating and responsive pattern of organisation. Abram (1996) reported that Māori culture aligns with a more ecological worldview, so its culturally sensitive significance was also important.

First, CAS is made up of a diversity of connected parts/nodes. When the interacting parts are the same, the system becomes skilled at addressing specific needs (Capra \& Mattei, 2015). Conversely, Capra and Luisi (2016) state that when the elements are diverse, the system learns to adapt to changing needs, making the same system more resilient and able to alter to contextual stimuli. Too many different components lead to chaos, while too few result in an inability to adapt (Boulton, Allen, \& Bowman, 2015a). Components that are same and different are both needed for a living system to sustain itself, but the connection of these adversaries is vital for survival. Links within a system are referred to as synergistic (Boulton et al., 2015a), and they construct the typical shape and boundary of the network.

Secondly, CAS use and reuse energy coming from, between and beyond the connected parts. Prigogine (1997) and Boulton et al. (2015) argue that living systems contain 
energy that is stable, and that flows. Each system not only generates its own energy which is used to self-generate a pattern within a boundary of its own making (closed system), but also results in a constant flow of unprecedented energy directed inwards from the environment and through a recognisable border (open system) which requires further adaptation. The energy can be naturally occurring or cultural, that is created by cultural norms. A living system will use available power, regardless of where it comes from, to continually move and grow.

Thirdly, feedback loops are recognisable in living systems as a means through which the system self-organises expected and unexpected energy and change. New circumstances, or information that needs to be integrated, sees the system react to change in ways that make adaptation possible or not. Cyclical or negative feedback loops describe reactions that sustain, self-generate and offer stability (Boulton et al., 2015a; Capra,1997) whereas developmental or positive feedback loops respond to disturbances of both internal dynamics and environmental change, and develop new structures in response (Capra,1997). Capra and Luisi (2016) see this process as a cooperative dance between renewing and reinvention since balance is not a static equilibrium. The term 'disturbance' (Capra, 2016) or what Boulton et al., (2015a) refer to as 'phase shift' (p. 99), allows movement to be noticed and responded to and indicates what is important to the person who notices the disturbance, as well as the people they interact with.

Fourthly, as energy ebbs and flows, CAS are forever confronting chaos. While developmental feedback loops continue, they self-amplify to create points of immense vulnerability, often referred to as bifurcations (Nicolis \& Prigogine, 1977), unfreezing (Lewin, 1976) or a tipping point (Byrne, 1998). At this critical point of variability, peak levels are possible (Adams, 2015). A level of engagement that sees a new qualitative form of order emerge to create a major shift (Boulton et al., 2015). The major shift creates an emergence of the new and is in fact, the most important discovery of complexity theory (Capra, 2016; Mason, 2008). Emergent change is evolutionary and is just as likely to be referred to in the literature as transformation. Transformational change creates new structures, new behaviours and creativity that can be both desirable and undesirable. A CAS, therefore, operates in the realm of volatility and imminent disorder from which the potential of transformative change emerges. 
Fifth and finally, the collective movement of energy creates a sum greater than its original parts. An understanding of the individual components and how they move and change the CAS cannot assume knowledge of the greater whole. Capra and Luisi (2016) describe this as 'holism'. As components become connected and interact continuously, each influences the other, generating a system whole that has characteristics that the individual components alone do not possess. Elements are part of the complex whole and are not divisible, with one undoubtedly always adding a contextual dimension to the other. The discrete components create ongoing movements that are so tied up in different parts of the system, that cause and effect scenarios establishing why disturbances and adaptations to the system whole occur are impossible to assume. A small number of people can make a difference that goes beyond any one of their capabilities. Negligible change, for example, can produce unreasonably significant consequences. Indeed, interactions are "non-additive" (Mason, 2008, p. 32). This means the whole does not equal the sum of the value of the parts. The accumulation of energy often produces changes that cannot be foreseen even at immediate range.

Discussing diversity, open and closed systems, equilibrium, feedback loops, disturbances, emergence and holism, has allowed me to highlight the origins of stability and change which are also the basic principles of ecology (Capra,1997). A complex system is that which has a diversity of parts, is continually moving energy to recycle or redevelop itself, and holds the possibility of transformation.

\section{Complexity theory and the social sciences}

Boulton et al. (2015a), Byrne and Callaghan (2013) and Cilliers (2002) advocate for the use of complexity theory in the social sciences. Although the natural and human world are both living systems (and mostly referred to as 'networks' in social sciences literature) focused on learning, there is an additional consideration when the network is socially bound - the application of meaning. Social systems are networks of communication and Capra (2016) believes that meaning is crucial to understanding these. From self-awareness and consciousness to language and culture, human systems are much more complex. How connectedness occurs, the meaning applied, and power is used, are the primary considerations for complexity in the social sciences. 
Complexity starts with understanding connectedness, and in the social world, this occurs through communication and relationships that identify how people and ideas relate to each other. Sanga states:

We rarely attend to the context behind the context - the spheres or domains of social relationships within communities. If we understand that, we will know much better what it is that we are trying to deal with (Sanga, cited in Airini et al., 2010, p. 11).

It is the social relationships that make up principal practice that this research is concerned with. Davies and Harré (1990) suggest that to position oneself within the language and social context is to allow for more fluid and evolving interactions between people and their conversations. Lowenhaupt (2014) argues that there is less direct attention in research and practice towards the role of conversations that act as a way of building connections or what Campbell refers to as building 'conversational communities'. Luhmann (1978) uses the term 'social autopoiesis' to describe networks of people who communicate thoughts, information, meaning and ideas that selfgenerate over time. Boulton et al. (2015) state that in the social world relationships can occur whether actions are adaptive or passive, intentional or unintentional, top down or bottom up, from people or positions of power.

Meaning is essential to humans' inner and outer worlds (Goleman \& Senge, 2014). Humans think ahead, communicate how they feel and make choices (Capra, 2016). The communication feedback loops formed in social systems result in both familiar and unfamiliar contexts of meaning. Shared meaning develops into culture (Capra, 2016). Stone and the Centre for Ecoliteracy (2009), add that community materialises when a familiar context of meaning is repeatedly maintained and renegotiated by the network. Capra (1997) observes that purpose is always related to context and is, in fact, an understanding of context itself. He adds that what we see, notice and respond to is not due to the volume or frequency of disturbances but how meaningful the disturbances are to the network.

Where achieving a shared context of meaning is impossible, power is used to resolve the disagreement or facilitate decision-making and is likely the most striking characteristic woven through the social world. Capra (2016) notes that power resolves conflict since differing beliefs and ideas often create conflicts of interest between people. Galbraith (1983) identified three types of power instigated by individuals: coercive power that intimidates for individual gain; compensatory power that offers a 
reward; and conditioned power that is influential and educational. Castells (2009), however, argues that the network itself is a source of power since there is power in both being connected and interconnected. Many questions arise that relate to network power. Who can or cannot be a member of the system? What stories and prior experiences are ignored or favoured? Who is supported and who is not? What change is occurring and whom does it endorse? Castells (2009) refers to networked power as empowerment. This type of power is used as a collective rather than individual source of decision-making and resolution, supporting the idea put forward by Capra (1997) that direct change is impossible since the living system itself decides how to change. Instead, the system can only be nudged (Capra, 2016). Power can either diminish the impact of the collective or enhance the ability of the collective.

When common meaning is agreed upon by the network, the changes occurring are likely to augment a shift in trajectory. When resolving agreement is not possible, change is expected to be destructive or constraining. Competing meanings appear to constrain social change.

\section{Complexity theory and education}

Several researchers in education have found complex thinking to be valuable (Byrne \& Callaghan, 2013; Cilliers, 2002; Davis \& Sumara, 2006; Mason, 2008) and its application is consequently widening. Areas of curriculum and school organisational management have used complexity theory (Davis et al., 2015; Senge, 2006; Uhl-Bien, Marion, \& McKelvey, 2007), as have education reform (Goldspink, 2007; Lemke \& Sabelli, 2008), education for at-risk populations (McGee, 2013), learning and change in schools (Morrison, 2010; Wielkiewicz \& Stelzner, 2005), and creating cultures of learning (Bendikson, Durie, Gilbert, Robertson, \& Wenmoth, 2016). The use of complexity in a variety of areas within education gave me some assurance of its relevance to educational leadership research.

I investigated studies which showed how complexity science had been applied explicitly to leadership. The studies mostly used complexity thinking to describe and/or explain the data gathered. Senge (2006), for example, used a sophisticated approach to examine the value of distributed leadership. Lumby and English (2009) used complexity theory to explain the added benefit of leadership programmes, while Uhl-Bien et al. (2007) attempted to apply complexity theory to expound how 
leadership enabled learning. Study findings included: an emphasis on the dynamic relationship between the bureaucratic and administrative functions of leading organisations, demonstration of how to conduct a process of problem identification and improvement, and an explanation of how leadership self-regulated. It appears that using both description and explanation to make vivid a phenomenon and construct a set of realities about that phenomenon could be considered as positively interdependent on each other. Positive interdependence between diverse ways of being is symptomatic of shared meaning and purpose.

Since emergence is one of the most important discoveries of complexity theory (Capra, 2016; Mason, 2008) and is the hallmark of life, development and learning, I focus here on what conditions have been identified in current educational research that might enhance the possibility of emergence and how leaders might further nudge or facilitate these possibilities. Davis and Sumara (2006) listed a series of conditions that would allow emergence to occur in educational organisations. Requirements included:

- a diversity of elements

- rich connections and coherence across the system

- an awareness of redundant components

- interacting ideas

- a redistribution of control

- following hunches and creating learning queries

- an appreciation of the regularity of randomness requiring the system to adapt

- a knowledge of the types of feedback loops occurring

- the management of failure

- $\quad$ sufficient means to preserve histories and identities

- the rate information flows through a system and the very size of the system

- an expectation of seeing errors. (p.135-136).

Furthermore, various authors discuss the implications of emergence for leadership and education reform initiatives. Uhl-Bien et al. (2007) suggest leaders can facilitate development by focusing on ways to incorporate new ideas, being comfortable with instability, self-doubt, uncertainty, or confusion, developing trust, showing a willingness to replace old structures with new and more meaningful structures, and choosing what is worth emerging. This perspective suggests leadership and context 
interact in a way that encourages learning as much as success and requires attention, thinking, dialogue, and action. While these aspects are useful to know, I am interested in the types of contextual disruptions that create barriers that might take interactions off course or conversely support them to remain on-task.

I was inclined not to describe leadership, as a single concept, but to use complexity theory to both define and explain principal educational leadership as a living complex entity, forever moving and changing. While the use of complexity theory in education is expanding, evidence of it in practical application is tentative. Jackson et al. (2017) suggest that showing leadership is one of the most challenging things in research. I required a design framework that would allow me to capture the evolution of principal leadership as a complex social system in tandem with an evolving social context. By and large, "one does not 'apply' complexity principles; one takes part in their articulation and elaboration" (Davis \& Sumara, 2006, p. 43). What the theory did not do was show how to organise the interactions between and within systems. It is illustrating the system that was noted by Eriksson et al. (2018) as most useful for guiding policy and practice.

\subsubsection{Holistic scientific theory}

The narrowest aperture of my theoretical lens provided a fine-grained, layered and nuanced understanding of the multi-dimensional influences on principal leadership practice in the LSE setting. As a documentary photographer would use a narrow aperture to capture everyday experiences, a holistic scientific theory might allow me to depict the eco-social action shot of principal leadership in practice.

Back in 1904, Dewey commented that to resolve education dilemmas it would be desirable to think about connections between theory and practice in novel ways. Lemke and Sabelli (2008) concur, suggesting what is needed is a broader conceptual framework for the analysis of education as a complex system, and argue that currently, education lacks the modelling capability needed to help practitioners and policymakers explore the potential impact of agency, power, knowledge, and autonomy. Boulton and colleagues (2015) point out that "Working out how best to undertake such 'real life' explorations is a work in progress" (p. 79). Modelling a complex system would allow for the 'how' of my research question to be more fully answered. 
Throughout my reading, the name Fritjof Capra kept appearing. Capra is an Austrian born American physicist who combined biological, cognitive, social and ecological dimensions to create a new perception on life, or what he refers to as a 'unifying vision' for understanding living systems. As a multidisciplinary conceptual theory, Capra's (1997) scientific view of life gave insight into a thriving living system forever in movement. Capra confidently argued that all living systems share similar concepts of structure, pattern and process and that these can be applied to a wide range of phenomena to gain a new perspective. To learn more about this conceptual framework, I took an online course with Capra himself in September through to December 2016. Three different but inseparable aspects of the phenomenon of life - structure, pattern, and process - helped me develop an understanding of the natural change process.

A structure is "the physical embodiment of the system's pattern of organisation" (Capra,1997, p. 161) and reveals a living system made of various components or elements. Byrne (1998) adds:

\section{We absolutely must relate containing systems to contained systems. In other words, we must have measures of individuals, of households, of neighbourhoods, of localities and so on, and we must recognise that we need to be able to relate all these levels to each other. (p. 10)}

As Byrne has shown above, leadership networks are distributed over many nodes ${ }^{18}$, the nodes being representative of individuals as well as formal and informal groupings and it is this immediate context I am exploring.

A structure can be both the outer form of a network as well as the internal framing that attempts to hold shape. Capra and Luisi (2016) describe interior framing that is both formally designed and informally, and socially emergent. Formal design structures include rules, policies and guidelines. These offer the organisation some stability and communicate what has come before. The informal arrangements in an organisation produce non-material structures or that which gives evidence of the culture or rules of behaviour, Parrett and Budge (2012) refer to these as mindset. Geyer and Cairney (2015) refer to informal structures as the sharing of norms and understandings. While requiring a delicate balance is required between the two, Capra and Luisi (2016) suggest it is the informal structures that hold the most creative potential and learning capability, or where the 'aliveness' (p. 318) of the organisation lies. Parrett and Budge (2012) argue that it is

\footnotetext{
${ }^{18}$ A node in the social sciences refers to a person or group.
} 
here that "indifference, ignorance, classism and racism, and at time anger or annoyance" (p.72) can also be evident. In any organisation, there is an interplay between the informal networks and its design structures. Capra and Luisi (2016) claim that "formal policies and procedures are always filtered and modified by the informal networks, which allow workers to use their creativity" (p. 317). Capra (2016) states that living systems decide what disturbances are noticed, so while the system does not decide on the changes, it does resolve which triggers will be noted and acted upon.

A pattern is the shape of the relationships that occur among the parts. Components generate reflexive interconnections, which in turn create discernible, although not necessarily predictable patterns of interdependence (Lewin, 1997). It is the patterns that are of most interest as they focus on the behaviours of the system proper (Morin \& Montuori, 2008; Senge, 2006) and reveal a system's essential characteristics (Capra, 1997). Like a memory of the future, Knyazeva (1999) advocates that patterns precede process. The emergence of a pattern is called self-organisation.

The process reveals what is created out of the reflexive relationship between the components and patterns (Capra, 1997). Exploring process focuses on the cognitive and autonomous coming together of a pattern (communication) and structures (social and design structures) and occurs as the response to uncertainty. The process focuses on how the system cognitively self-regulates for both continuations of the same and emergence of new. Capra states:

Cognition, according to Maturana and Varela $^{19}$, is the ability of adaptation in context and involves the entire process of life including perception, emotion, behaviour, language, conceptual thought. A process changes something from one state to another. (Capra, 2016, Course notes)

When internal dynamics or the environment trigger disturbances, the system itself determines both what to notice as well as how to respond and can either be changes of self-review to maintain stability or changes of development (Capra, 2016). The process identifies the coming together of the learning trajectories and the tensions, or how the network makes changes to stay on course. Self-organisation is not designed by an external agency, cannot be predicted, and occurs through repeated nonlinear interactions of individuals producing a changed pattern.

19 Maturana and Varela were biologists responsible for coining the term autopoiesis, a term used to describe the phenomenon of living organisms. 
Meaning is the fourth dimension added by Capra when the living system is human. Meaning is essential to humans' inner and outer worlds. While individuals have dependent thoughts and ideas or agency, they are also continually influenced by others' thoughts and actions. Berger (2004) refers to a human-centric system as one that is always "dancing on the threshold of meaning" (p. 336). Additionally, Capra (2016) states that meaning is always related to context, an understanding of context even.

Capra has applied his conceptual framework to various disciplines including law (Capra \& Mattei, 2015), business (Capra \& Pauli, 1995), politics (Spretnak \& Capra, 1997) and art (Capra, 2008). He uses the framing for three main purposes: first, to show the importance of contributing to social evolution; second, to refocus on solutions that are ethical, sustainable, and successful for the inhabitants of the world's biosphere; third, to display how systemic thought can broaden one's scope. For example, in healthcare for diseased patients, instead of using a biomedical model which requires looking to the origin of a disease and the process through which diseases manifest, he suggests instead looking at the process of healing, and gaps in this process (Capra, 2016). Capra has used his conceptual framework to change perceptions, which he says is the current and most relevant crisis of our time (Capra, 1997).

Many aspects of Capra's ideas appear within work relating to leadership, for example, work by Chatwani (2017) on distributed leadership; and Klimek et al. (2008) on generative leadership. To date, there appears to be no educational leadership literature that has applied Capra's conceptual tool to organise and analyse data. The work of Raelin (2016) that promotes leadership as an "architecture of movement" (p. 216), that unfolds and emerges moment by moment, day-upon-day, is the closest I could find.

The scientific systems' view of life (Capra, 1997) offered me an open and holistic framework through which to describe, explore and explain Complex Systems Theory and principal leadership practice simultaneously. The four main concepts outlined above (structure, pattern, process and meaning) would allow data to be gathered, analysed and discussed holistically, showing principal leadership as a moral and ethical eco-social process, always in movement, that is not only able to deal with the complexity of the environment but is also ecologically sustainable (Capra, 2016). This 
would identify qualitative growth as that which boosts the quality of life through reproducing and reinventing itself and is essential for the sustainability of living systems (Capra \& Luisi, 2016).

\subsection{Chapter summary}

This chapter explained how I developed the theoretical framework for this study using the metaphor of the aperture of a camera. I started with Bronfenbrenner's ecological systems theory, and used this as a springboard for focusing on people and context. Next, complexity theory was used to focus on the interrelationships occurring between the two. A refocus on the in-between implied a shift from analytical thinking to contextual thinking. The natural movement and change that arose in the process exemplified principal leadership as a living system. Finally, I drew on Capra's systems view of life. Capra's new scientific understanding of living systems provided framing for developing sophisticated holistic exploration of leadership as an eco-social process making the all-important link between theory and practice. Together these three theories acted as the foundation on which to build a methodology to show principal leadership in practice and how it supports qualitative growth for students in an LSE context. 


\section{CHAPTER 4 \\ The Methodology}

"Tell me what the ends are, and I will tell you if the means are OK"

Guy Claxton

\subsection{Chapter overview}

Having reviewed the literature and current knowledge gaps, and decided on the theoretical framing, the methodology is now explained. Methodology is the "theory of how researchers gain knowledge in research contexts and why" (Briggs, Coleman, \& Morrison, 2012, p. 15). The why establishes the research questions, problem and objectives. The how of the investigation involves an explanation of the choice of approach, researcher stance, research design, and the suitability of an embedded, emergent exploratory case study. Information on research implementation includes an outline of participant recruitment, data sources, methods analysis and measures to ensure the ethics and trustworthiness of the study. How the findings will be arranged concludes the chapter.

\subsection{Research questions}

The introductory chapter identified the research focus as that of the achievement gap and the need to investigate in greater depth the influence principals have on this issue. The literature review included relevant aspects of educational leadership: positioning, attributes, purpose, performance, and context, as well as the implications of these components on principal leadership. Additionally, an emerging theoretical framework explored the research topic and identified the practical considerations for considerations. The overarching research question was:

How do principals practise leadership in a NZ LSE primary school context for educational success?

In this study, the question explored principal leadership within the NZ context, implying that effective education reform is not just about how policy but how we choose to enact the reform. This central question led to four sub-questions, each intent on exploring different aspects of the gaps in the literature: 
1. What leadership practice patterns are in evidence in the daily life of a LSE principal?

According to Friedrich et al. (2016), research that explores principal leadership as both the individual and the collective is rare, Shields (2003), influenced by Wheatley and Kellner-Rogers (1998), suggests too little time is spent understanding patterns. The design of the study allowed for a hybrid conceptualisation of leadership, as a role, group activity and a pattern of connections that explores how movement, stability, and change occur. These are essential aspects of leadership as a living system and thus leadership in practice.

\section{How is LSE principal leadership influencing of, or influenced by, context?}

This question highlights the LSE context, keeping it integral to the data analysis and findings so that the natural change process becomes apparent. Issues of the relationality between underachievement and poverty involve every part of society, so broader communities of practice would need to be mobilised.

3. What beliefs, values and ideas underpin principal practice patterns in the LSE context for educational success?

The literature review argued that there are varied purposes for the actions of leaders. This question intended to highlight how a principal's purposes and the purposes of the network interdependently influence the focus of student outcomes in a LSE primary school NZ setting.

\section{What do the stakeholders in the LSE environment perceive as educational success?}

The literature review recognised that how people perceive educational success contributes to what they learn, how learning is measured, and the value placed on what the school is doing. Thus, this question considered localised versions of success that enhance the quality of life through generation and regeneration from within the local culture. 


\subsection{Research problem}

There is no clear picture in current literature of the complexity of principal leadership and practice patterns that emerge in the LSE context for enhancing localised notions of success in NZ.

\subsection{Research approach}

Research conventionally aligns with positivist/post-positivist, constructivist, transformational or pragmatic paradigms (Guba \& Lincoln, 2005). What follows is an explanation of why this study has drawn on compatible elements of both the constructivist and transformational paradigms specifically. The ontology or the nature of reality, epistemology or what truth is and the methodology or the research process (Creswell, Hanson, Clark Plano, \& Morales, 2007) are outlined.

The constructivist paradigm states that when individuals interact with others in context, personal knowledge is made (Carr \& Kemmis, 1986). What also results, is therefore multiple perceptions of reality emerging in motion and context (Mertens, 2014). This research set out to gather the multiple perspectives on the many who involve themselves in principal leadership networks. The research network was constructed by the principal participants in motion. The research design anticipated variation in the network involvement as well as in the definition of education success and there was room for these to develop as the research progressed. Thus, multiple perspectives were anticipated and valued.

The transformational paradigm was also necessary, since the constructivist paradigm was not developed to challenge ideas and my desire to hear directly from those living and working in the LSE context led me to look to a theoretical framework that goes beyond personal and socially constructed truth claims to how the impact of power relations privileges one group's knowledge over another. As a researcher working within a critical reality, I was primarily concerned with "influences of a political, social or ethical nature, emphasising a variety of diverse voices from the margins" (Mertens, 2014, p. 21). The research served to bring an authentic description of marginalised learning communities shaped by socio-economic disadvantage. The research was particularly mindful of aiga/whānau noted in the literature review as a vital support mechanism for increasing the performance of students living in the LSE setting yet one of the most challenging to engage in research (Gordon, Jean-Louis, \& Obiora, 2018). 
Identified in the literature review was the idea that leadership has become more of a technical task than a human one, relegating the realities of context to the shadows, making it hard for principals practising in a LSE contexts to gain traction.

Both the constructivist and transformational paradigms acknowledge the contribution of context (Creswell, Hanson, Clark Plano, \& Morales, 2007). In this research the focus on schools and principalship saw the research investigate leadership as a living ecosystem. Living systems have subtleties and reveal persistent patterns, repetitive properties and processes in response to context. They resist change, but they also adapt and evolve. Capra (1997) refers to principles of ecology as recognising interdependence, cooperation, and partnership since this is how nature has survived for millions of years. Understanding how systems maintain themselves naturally had practical consequences that went to the heart of leadership for social justice. Just like ecology, leadership networks are positively interdependent on both people and context, where individuals are expected to come together for some shared understanding of collective good, making sustainability and even transformation likely.

\subsection{Researcher stance}

Most research involves taking on a defined researcher role, giving the researcher the power to collect, classify and represent findings (Smith, 2005). I reflexively revisited the following types of questions proposed by Smith to help guide practice throughout:

- to whom does the research belong?

- who owns it?

- whose interest does it serve?

- who will benefit from it?

These questions ensured I was aware of undue influence. The research was owned by both the researcher and the researched but was primarily a story of participants and their experience of leadership in a local LSE context, and so I needed to work to avoid my own bias and to build a research agenda that was mindful of participants. 
With power and purpose in mind, the insider/outsider researcher roles in this study became fluid. I maintained an ongoing awareness of the effect I had on others and the situation, and which others and the situation had on me as a research instrument. When I entered the research site I was an outsider in that I did not know the context. However, previous experience as a facilitator allowed me to research 'with' rather than 'on' others (Berryman et al., 2013), quickly making me more of an insider. At times, I acted as a mentor to principals by assisting them to apply leadership theories to their work, and reframe perceptions for enactment. Active roles such as facilitating the interviews helped me to acquire an "insider" understanding of the phenomenon under study as referred to by (Schwandt, 2000, p. 102). When analysing data, I was both an insider and outsider.

\subsection{Research design}

Case study research has contributed significantly to researchers' knowledge of educational leadership (McNae et al., 2017; Lárusdóttir, 2014) and was viewed as a likely suitable strategy for this research.

Firstly, the use of case study offered a chance to explore a contemporary, real life issue (Yin, 2014). The introductory chapter described decades of reform focused on the correlation between LSE status and underachievement. Significant change that amounts to the progress required to narrow the achievement gap but also change the pattern, remains elusive. People, groups, communities, towns and even countries have not yet solved this issue making it an imperative issue for consideration. In addition, the literature review revealed that a rich description of the interaction between LSE context and principal leadership is a neglected focus of educational leadership literature in NZ. The case study design would allow me to comprehensively study a modern-day phenomenon from within the NZ context, to contribute to an understanding of how to narrow the underachievement gap and change patterns and the change trajectory.

Secondly, case studies are useful for "appreciating the complexity of organizational phenomena" (Yin, 1994, p. xv). The problem, which was a multifaceted and interconnected problem with no distinct origin and no clear solution, required a reframing via multiple forms of evidence, from multiple stakeholders and with the 
wider system for which they were a part in mind. Systems thinking offers a more holistic view on the modern issue of underachievement by looking at the overall effects principal leadership practice. Yin (2018) argues that case study is "the preferred strategy when 'how' or 'why' questions are being posed, when the investigator has little control over events, and when the focus is on a contemporary phenomenon within some real-life context" (p.1). Exploring the systemic influences occurring between the leader, people and place, would result in a focus on the complexity of how practice evolved.

According to Gall, Gall and Borg (2007), planning a case study requires a clear vision of the phenomenon, case, focus and unit of analysis. Gall et al. (2007) separate these elements as case layers that assume higher levels of abstraction. Table 3 below shows how I adapted their ideas to this study.

Table 3: Key case study elements adapted from Gall et al. (2007)

\begin{tabular}{|l|l|l|}
\hline $\begin{array}{l}\text { Phenomenon } \\
\text { A process, event, person or } \\
\text { item of significance }\end{array}$ & $\begin{array}{l}\text { Application to this study } \\
\text { Leadership practice }\end{array}$ \\
\hline Case & $\begin{array}{l}\text { An instance of the } \\
\text { phenomenon }\end{array}$ & $\begin{array}{l}\text { Application of leadership practice in } \\
\text { an LSE setting }\end{array}$ \\
\hline $\begin{array}{l}\text { The centreing of data } \\
\text { collection and analysis }\end{array}$ & $\begin{array}{l}\text { The movement and change occurring } \\
\text { when principal leaders practice } \\
\text { leadership in a LSE setting for } \\
\text { educational success }\end{array}$ \\
\hline Unit of analysis & $\begin{array}{l}\text { Aspects to be studied across } \\
\text { the case }\end{array}$ & $\begin{array}{l}\text { Two principals and their principalship } \\
\text { within the school context }\end{array}$ \\
\hline & & \\
\hline
\end{tabular}

With the case, focus and unit of analysis centred around the development of complex developing leadership behaviours, it seemed important to acknowledge emergence. An emergent case study design, "begins with a loose participatory framework, which is utilised to define the roles and interactions of those involved, but not to prescribe the evaluation process as a whole" (Christie, Montrosse, \& Klein, 2005, p. 271). Who and what was studied was sufficiently bounded by the network. The network was also open enough to allow me to adapt the process as required. By treating the research process not as absolute (Mertens, 2014), but as emergent, I acknowledged the complexity of the work before me. 
The units of analysis would be brought together, making this case study embedded. Embedded case studies comprise more than one sub-unit of analysis (Yin, 2018) and allow for a more comprehensive level of inquiry. Lyotard (cited in Cilliers, 2002) argues for applying a narrative understanding of smaller stories that function well in context. A narration of effective principal leaders in the wider educational leadership context created smaller stories of primary school leadership in the LSE settings.

Yin (2014) argues that the best types of case study approaches are unlikely to be as straightforward as exploratory, descriptive and explanatory. An exploratory case study tends to result in observing and describing theoretical ideas (Gibbs, 2012), a detailed case study attempts to build on exploratory research and explains how (Yin, 2014), and an explanatory case study tries to build on both exploratory and descriptive and explains why things happen (Yin, 2014). This research had two critical points of interest to justify the use of descriptive and exploratory approaches combined.

First, in the findings chapters, I describe the principal leadership network and then in the cross case-analysis and discussion, explore the complex nature of principal leadership as patterns of practice created by feedback loops, within the system whole. I broaden the explanations in the discussion chapter by further explicating in complex terms, the capability of the principal leadership network overall and whether the network is likely to be sustainable. By mixing descriptive and exploratory case study design features, I can describe what principal practice is and how it occurs.

\subsection{Research implementation}

This section explains how the research was carried out; how participants were recruited, how data were sourced, and the research methods used. I then discuss how the data were organised, analysed and how ethical responsibilities and study trustworthiness were safeguarded during the inquiry process. 


\subsubsection{Participant recruitment}

Recruitment occurred in two phases. First, the researcher recruited the principal and the school and then enlisted all other participants with the help of the principal.

\section{Phase one}

Criterion sampling was used to select the principal leadership network. Patton (2002) describes criterion sampling as the setting up of criteria by the researcher that cases must meet to be involved. Units for analysis would:

- represent a range of LSE schooling environments in NZ that include state and integrated, urban, and schools of various sizes. Since smaller schools are the most common indicated in the literature review, schools with less than 100 students were especially considered

- have the highest possible deprivation index scores and lowest possible school decile since together these were the best available indication of a LSE context

- have high Māori/Pasifika student populations, a diverse ethnic mix and a shifting roll. These were aspects of a schools' demographics, that have been identified as associated with those in the tail of underachievement

- include principals acknowledged as successful in their leadership by others. This included schools led by a principal who maintained a positive peer reputation.

It also included principals who had been at their schools long enough to receive encouraging external ERO reviews (these were available on-line) that could, in part at least, be attributable to them. Choosing principals already deemed successful in LSE school contexts should show how principals who are effective are already engaging to enhance educational success, despite the challenges of the schooling system

- include principals who had been in their school for at least five years, long enough for the establishment of school procedure, leadership processes, and the school culture to be influenced by their leadership.

An internet search yielded valuable information about NZ schools and school principals. Merriam (1998) comments that this is useful before beginning fieldwork. A full list of all primary schools across NZ available from the MOE website was filtered using relevant public information on community deprivation index scores, 
school decile, and school population. Further, the NZ MOE information held on the school, such as its ERO reports, was valuable in ascertaining whether a school met the research criteria.

Additionally, discussions took place between the researcher and individuals with an in-depth practical and theoretical knowledge of the NZ primary schooling context which included: an ERO representative, a university lecturer, a school consultant, and an experienced researcher and principal. These 'experts' could, through their firsthand knowledge, help to eliminate any schools and principals that did not meet the criteria and were perhaps not evident in official documentation, for example, if a principal was no longer at a school or the school was already involved in research.

Eventually, seven schools and their principal leaders were identified. The principals were approached first by email/ phone and then face to face. This initial contact introduced the researcher and the study. At the end of the meeting, I left an information sheet with the principal (Appendix C), along with an example of the research schedule (Appendix D) so the time commitment was evident. Principals were asked to register their interest with a follow-up email or a phone call.

Of the seven schools and their principals initially identified, one decided not to proceed, two had to withdraw from the research due to issues related to community safety and volatility, and one school was too great a distance from the research base to be practicable, leaving three schools that could accommodate the research. Table 4 below offers an overview of the three schools initially included in the study. Data were sourced from Statistics NZ or local council records. 
Table 4: Overview of the schools chosen for this study

\begin{tabular}{|c|c|c|c|c|c|c|c|c|}
\hline School & Principal & $\begin{array}{l}\text { Principal } \\
\text { tenure } \\
\text { period }\end{array}$ & Region & $\begin{array}{l}\text { School } \\
\text { roll }\end{array}$ & Decile & $\begin{array}{l}\text { Community } \\
\text { make-up }\end{array}$ & $\begin{array}{l}\text { Deprivation } \\
\text { index } \\
\text { score }\end{array}$ & Other \\
\hline $\begin{array}{l}\text { Acacia- } \\
\text { Integrated } \\
\text { Catholic }\end{array}$ & Amber & $6-9$ years & $\begin{array}{l}\text { North } \\
\text { Island }\end{array}$ & $\begin{array}{l}95-105 \\
5-13-\text { year- } \\
\text { olds }\end{array}$ & 1 & $\begin{array}{l}\text { - Pacific } 72 \% \\
\text { - Măori } 10 \% \\
\text { - Other ethnic groups } 14 \% \\
\text { - NZ European/Päkehā } 4 \% \\
\text { - } 80 \% \text { ESL } \\
25 \% \text { of adults unemployed and } 50 \% \text { in low-paying jobs } \\
\text { - } 28 \% \text { of the population own their homes } \\
\text { Average income } \$ 22,000 \text { compared with } \$ 49,000 \\
\text { nationally }\end{array}$ & 10 & $\begin{array}{l}\text { Outer city suburb of } \\
38,000 \text { surrounded by } \\
\text { poverty } \\
\text { State houses } \\
\text { demolished reducing } \\
\text { school roll by } 30 \%\end{array}$ \\
\hline Birch state & Baker & $6-9$ years & $\begin{array}{l}\text { South } \\
\text { Island }\end{array}$ & $\begin{array}{l}196 \\
5-13 \text {-year- } \\
\text { olds }\end{array}$ & 4 & $\begin{array}{l}\text { - NZ European/Pākehā } 63 \% \\
\text { Mãori } 27 \% \\
\text { Pacific } 5 \% \\
\text { - Other ethnic groups 5\% } \\
\text { - } 1 \text { pproximately } 55 \% \text { ESL } \\
72.5 \% \text { of families are in full-time employment } \\
\text { - } 68 \% \text { of the population own their own homes } \\
\text { Median household income } \$ 54,300 \text { compared with } \\
\text { national of } \$ 63,800\end{array}$ & $\begin{array}{l}\text { Range } \\
1-10\end{array}$ & $\begin{array}{l}\text { A provincial coastal } \\
\text { suburb of } 46,000 \\
\text { surrounded by } \\
\text { affluence } \\
\text { Housing increased } \\
\text { school roll }\end{array}$ \\
\hline Cedar state & Cathy & $6-9$ years & $\begin{array}{l}\text { North } \\
\text { Island }\end{array}$ & $\begin{array}{l}190-200 \\
5-13-y e a r- \\
\text { olds }\end{array}$ & 1 & 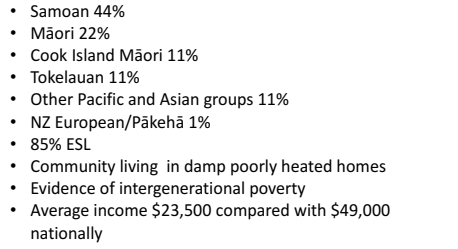 & 10 & $\begin{array}{l}\text { Outer city suburb } \\
\text { of } 54,000 \\
\text { surrounded by } \\
\text { poverty }\end{array}$ \\
\hline
\end{tabular}

As the theory aperture became more refined, I recognised I did not have the resources needed to analyse three schools to the depth required. In preparation for the intensive data analysis and findings stages, I decided to proceed with only two of the schools. With little difference between the principals, but some differences in the school makeup, Acacia and Cedar Schools were included in the final thesis as they most closely met the principal and school criteria established for the research.

\section{Phase two}

In phase two of recruitment, principals invited participants to the study who would best represent their principal leadership network/s. Three main groups were: staff, students, and the community (inclusive of parents).

Teaching staff were encouraged by the principal to participate. At Acacia School, the interviews were held in a staff meeting and were attended by all staff. At Cedar School, five out of eight classroom teachers attended the interviews.

Both principals asked classroom teachers to consider recruiting their class for the research. The teachers contacted me by email if this interested them. At Acacia School the senior students participated, and at Cedar, middle school students. Both the teacher and all parents signed the consent forms on behalf of students. 
By far the hardest group to recruit was the aiga/whānau. Successful recruitment was reported by other aiga/whānau as unlikely due to a lack of availability and confidence in the value of their voice in research. Some aiga/whānau members were recruited because they were frequently present at the school and showed an interest; while others responded to a school newsletter request. Still others were approached by the principal and asked to consider being involved. I also made myself available whenever I was on site and advertised my availability on the school noticeboard. Such adaptability was required so that minority voices were recruited and could be heard (Gegeo \& WatsonGegeo, 2002). Aiga/whānau were given petrol and food vouchers for their participation after the data analysis stage.

The community was the most diverse group for recruitment. Similar techniques to those used above were employed, with snowballing a technique noted by Denzin and Lincoln (2011) additionally used. Snowballing occurs when existing study participants recommend further research participants. In the end, education support workers, health and welfare services, and private businesses and organisations represented the wider community.

\subsubsection{Data sources}

The two primary data sources were people and documents.

People

I provided each principal with a draft of the Bronfenbrenner framework (1979). The chrono-system represented the principal leader, their upbringing, personal philosophies, early career and leadership journey. The principal drafted the remainder of the ecological leadership framework and included what they believed the purpose of each network was. The principal practice networks included:

- a micro-system renamed Network 1. This network represented people who had the most direct relationships and interactions with the principal within their immediate school surroundings. Principals elected to include their leadership team, teaching and support staff, and students.

- a mesosystem renamed Network 2, connected two or more systems in the neighbourhood or learning community. Principals elected the BOT, aiga/whānau, and education support workers. Relationships represented here are less regular but 
directly support the teaching and learning.

- an exo-network or Network 3 defined the more extensive social system with which the principal interacted. Principals elected to include private businesses and organisations. Described at the beginning of each of the findings chapters were aspects of the wider city and community.

- a macro-network was mostly described in the thesis introduction and included current nationwide political agendas and social environments influential in education reform initiatives, associated policies, leadership guidelines and PLD, and school funding and operational considerations.

For simplicity, the term 'network' is used to describe what Bronfenbrenner (1979) referred to as systems. Systems within these systems will be referred to as Nested Networks (NN). Table 5 shows the principal leadership network membership and sphere of influence.

Table 5: The transferal of systems to networks

\begin{tabular}{|c|c|c|}
\hline $\begin{array}{l}\text { Bronfenbrenner } \\
\text { system/s }\end{array}$ & Network & Nested networks \\
\hline Chrono & Principal & $\begin{array}{l}\text { Upbringing } \\
\text { Early career } \\
\text { Leadership journey } \\
\text { Personal philosophy }\end{array}$ \\
\hline Micro & 1 & $\begin{array}{l}\text { Leadership team } \\
\text { Teaching staff } \\
\text { Support staff } \\
\text { Students }\end{array}$ \\
\hline Meso & 2 & $\begin{array}{l}\text { Governance } \\
\text { Aiga/whānau } \\
\text { Health and welfare services } \\
\text { Education support workers }\end{array}$ \\
\hline Exo & 3 & $\begin{array}{l}\text { Private businesses and } \\
\text { organizations }\end{array}$ \\
\hline Macro & 4 & Wider policies and systems \\
\hline
\end{tabular}

\section{Documents}

Documents can be a variety of items from agendas to reports (Yin, 2014) and were the second data source of importance. While a list of possibilities was predetermined, the principals were asked to elect documents that would best show evidence of their leadership practice. Permission for document use was sought from the BOT or directly from individuals or groups that created the document. Both principals chose the school charter, but Amber additionally included the school's behaviour policy and teacher 
appraisals. Additional documentation that supported formal observations or interviews surfaced. For example, when observing a leadership meeting, both principals later offered me the minutes from that meeting.

\subsubsection{Data collection methods}

The research methods used to examine the work of the principal leader are predominantly qualitative and included observations, both formal and informal, and interviews, both in-depth and semi-structured. These took place when and where suitable and most preferred by network members. The exact timeline for this process is evident in Table 6. 

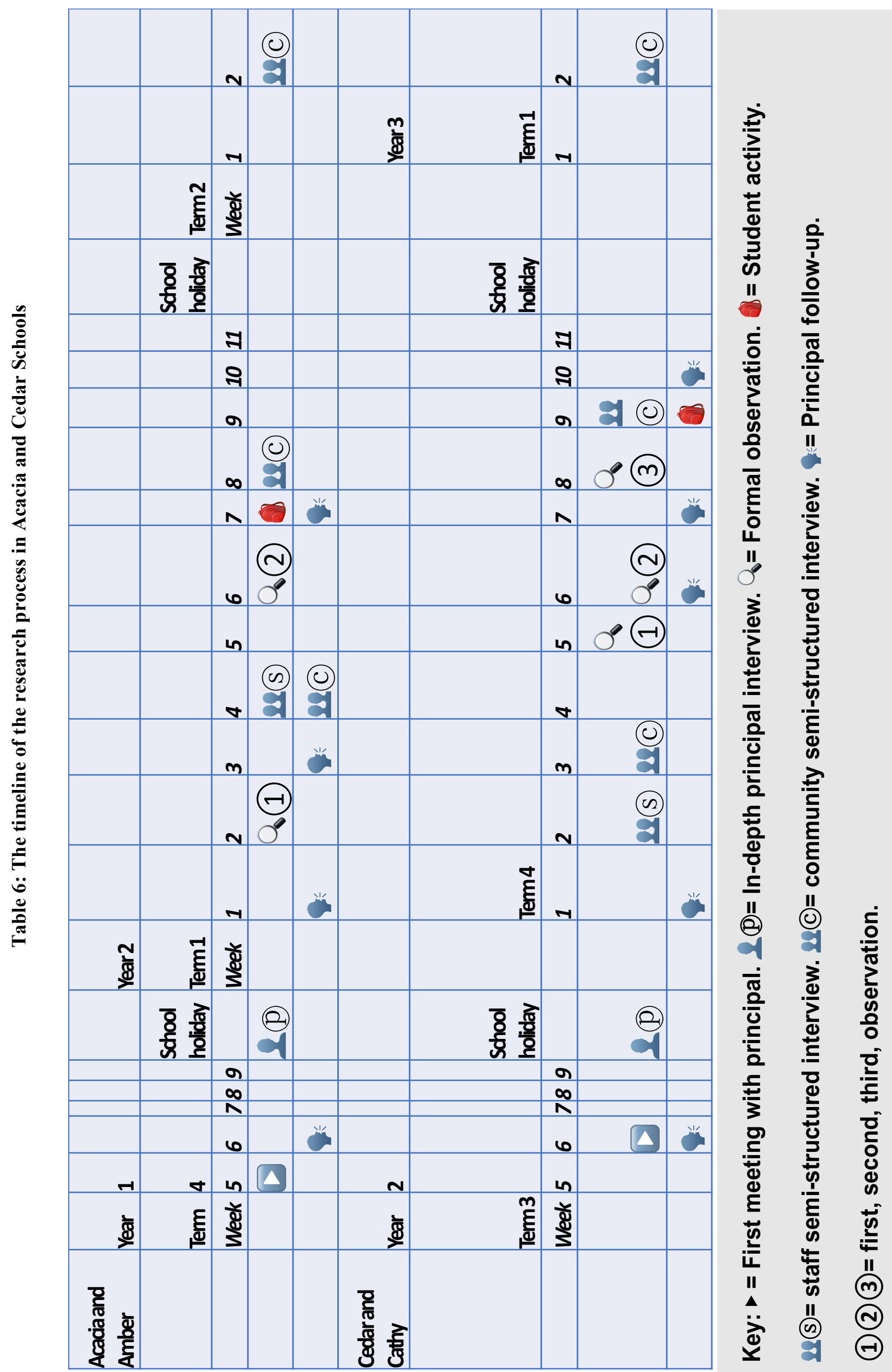


\section{Observations}

Observations can be useful for studies of leadership and management and allow for data that are grounded in actual experiences (Briggs, Coleman \& Morrison, 2012). Observations were mainly used to capture authentic examples of who communicated with whom and about what.

\section{Direct observations}

A maximum of three direct observations of each principal were undertaken to get a picture of principal leadership practice patterns. Direct observations offered a chance to gather data in a 'real world' setting to see how practices looked and sounded.

Observation one was a shadowing day. The principals had identified their networks, and the observation helped me gain a fuller understanding of the components involved in principal leadership and confirm or adjust the principals' draft. Principals were given an opportunity to make a time-diary ${ }^{20}$ of their day. In both cases, the principals opted for the researcher to shadow them and gather the data, as they both felt this would reveal more. It should be noted that because of ethics requirements for recruiting participants, data gathering possibilities were limited. According to Briggs et al., 2012, all research participants should be supplied with adequate information about the research and be given the opportunity to say no, without undue pressure. Since participants materialised as the day unfolded information about the research and an opportunity to refuse participation, was not possible to arrange, without encumbering the natural rhythm of a principal's day. Therefore, I focused only on the principal during the shadowing day and noted down each activity they were involved in and/or general topics of conversation they instigated. The principals and I jointly decided on the protocol for this day which included no interactions with the researcher and involved asking the principals to speak their thoughts and actions when working alone, so the activity worked on became apparent to the observer. Where there were lengthy conversations with individuals not initiated by the principal, the researcher removed herself or was asked to leave to maintain privacy.

20 A time diary is defined by Robinson and Godbey (2000) as "a micro-behavioural technique for collecting self-reports of an individual's behaviour in an open-ended fashion on an activity-byactivity basis" (p 66). 
Observations two and three were formal. Both principals elected two activities from a menu of possible activities. These were activities they might be actively engaged in, and considered were representative of their leadership practice and part of business as usual. In both cases, I observed a BOT meeting and leadership team meeting. Members of these meetings were informed about my presence well in advance and were given the opportunity to opt out, although none chose to. Audio-recording and/or videoing allowed me to review the observational experiences. Observations two and three were transcribed to ascertain topics of communication and how the conversation emerged.

\section{Indirect observations}

Maintaining a researcher journal is recommended as an integral component of qualitative research design to enable researchers to record their impressions, insights and reflections throughout a project (Maykut \& Morehouse, 1994). This strategy recognises that case study researcher thinking is reflective (Stake, 2006) and an essential part of the research process. I took field notes which included information about "who was seen, when, where and why" (Briggs et al., 2012, p. 167). I also included environmental conditions, contextual aspects, programmed and unplanned activities, and participant behaviours. I did, however, use coding when referring to specific people. Notes were not checked by participants but were made available on request. An example of field notes can be found in Appendix E.

\section{Interviews}

Interviews are noted by Cohen et al. (2017) as imperative and one of the essential methods of case study information. Three types of interviews were used to collect data: in-depth semi-structured principal interviews, semi-structured group interviews, and the student interview.

\section{In-depth semi-structured principal interview}

In-depth semi-structured interviews were planned for the principals. I gathered data on historical events in a principal's life to elicit the interviewee's knowledge or perspective on the topic of leadership practice for educational success, and explored associated beliefs, values, experiences and perspectives. Conversation starters included:

- tell me about your leadership journey

- tell me who or what has impacted your leadership journey 
- tell me about a day in your life as a principal leading this school

- describe an "aha" moment that let you know you had succeeded in your work.

Questions included:

- what are you good at and how do you know?

- what has changed since you have been at the school?

- what are the deliberate things that you have done in response to context?

- what does educational success mean to you?

The semi-structured principal interview schedule is presented in Appendix F. The interviews lasted between 1.5 and $2.5 \mathrm{hrs}$, as determined by the principal. Both principals elected to be interviewed in their school office. These interviews were audio-recorded and transcribed. Taking notes while I was there could have been an option, but this would have meant I could not be fully engaged as a facilitator of the interviews and risked missing essential details.

\section{Semi-structured group interviews}

Semi-structured and often group interviews were held with members of the staff and community to explore the varied perspectives on leadership practice for educational success. Café-style interviews allowed me to create a safe and comfortable interview space, where collectives of diverse people could discuss a topic. The World Café approach (Brown, 2005) was used with classroom teaching staff and the community (including parents). Café etiquette was co-constructed with participants before the session began. Elements included: listening to understand and asking more in-depth questions. Each session was set up like something of a pop-up ${ }^{21}$ café with tea/coffee and food on each of the tables.

Each small group split into smaller groups and discussed an aspect of the research. Context, leadership practice, and educational success were the three main aspects, and questions included:

- Tell us about your school? What's great about it and how do you know? How could it improve?

\footnotetext{
21، Pop-up' is a contemporary term used to refer to a short-term retail event.
} 
- Tell us about principal leadership at your school? What is great and how do you know?

- What is the principal leader good at and how do you know?

- What does educational success look and sound like to you?

A sample semi-structured interview schedule for the various participant groups, is presented in Appendix G. Rounds of conversations took place for approximately 1520 minutes each so that ideas could be shared and developed with others and, "after several rounds of conversations, themes and ideas begin to emerge and crosspollinate" (Brown, 2005, p. 113). The NN representatives came together as a large group, to summarise their thoughts and ideas. Each NN had a recording device located at their table. These sessions were later transcribed.

With some subtle changes to adapt to the group size and make-up, all café-style groups worked well and had the desired effect of naturalising the environment at Acacia. However, at Cedar School, café-style interviews were abandoned with staff. At Acacia, the 'setting-up' of the café had made the environment somewhat contrived. At Cedar, I instead set up in the staffroom, much like the principal would for a staff meeting. Additionally, the staff requested they talk together as one large group rather than in smaller groups. The alternative interview format was agreed to because it was again familiar to the staff and was considered likely to positively influence participant contributions.

Hearing the voices of aiga/whānau was an essential goal in the research. While the initial hope was that the research would give a voice to the LSE school communities, in fact, the power within the school culture and between people was more likely to determine who would speak. Gallagher (2008) refers to this as dispersed power. Power is not always overt, and having a diverse range of community members present, did not always guarantee the participation of all. I overcame this to some degree at both Acacia and Cedar by agreeing to regroup participants. Some aiga/whānau participants were happy to be a part of a small mixed group, and some aiga/whānau participants preferred one-to-one interviews. Still others chose to be part of a self-selected group (Samoan parents). The final regrouping of the research participants is outlined in Table 7 below and shows the total numbers of staff and community interviewed at each site. 
Table 7: Staff and community interviews that emerged across the case study

\begin{tabular}{|c|c|c|c|c|c|}
\hline School & $1-1$ & $\begin{array}{l}\text { Self-selected } \\
\text { group }\end{array}$ & $\begin{array}{l}\text { Café-style } \\
\text { interview }\end{array}$ & $\begin{array}{l}\text { Number } \\
\text { interviewed }\end{array}$ & $\begin{array}{l}\text { Total staff and } \\
\text { community } \\
\text { interviewed }\end{array}$ \\
\hline Acacia staff & - & - & 1 & $\begin{array}{l}6 \text { out of a } \\
\text { possible } 7\end{array}$ & \\
\hline Acacia community & 1 person & $\mathrm{Nil}$ & 1 & 6 & 12 \\
\hline Cedar staff & - & 1 & - & $\begin{array}{l}6 \text { out of a } \\
\text { possible } 8\end{array}$ & \\
\hline Cedar community & 2 people & 1 & 1 & 8 & 13 \\
\hline
\end{tabular}

Regardless of where the interviews took place, and who was present, the discussions allowed me to explore a range of opinions and ideas and the inconsistencies and variations that existed.

\section{Student interviews}

Student interviews took the form of an in-class activity. The researcher predesigned the activity and adapted and/or changed it once consent from the classroom teacher and parents was confirmed. The activity was adapted in both instances so that it was cooperative in nature and suitable for the class of eleven and twelve-year-olds at Acacia School and eight and nine-year-olds at Cedar School. Only students who had signed permission slips from their parents had their ideas recorded. Further, consent was sought from the classroom teacher to use anything created during the activity.

A literacy task was set for students to create an advertisement for a new school principal. Thinking of their current school principal, they were asked to create a timetable for a typical principal's day and discuss what the principal would need to be good at and be able to do, what school success they would want to share with a principal interviewee, as well as what they were working on to get better. Each table transcribed a principal's day onto a sheet of paper and then added detail as it arose in conversation. The groups rotated their advertisements to check what others had written and to gather different ideas. The activity concluded with the whole class coming to a shared consensus about aspects of principal leadership practices and educational success. 


\subsection{Data analysis}

As indicated in the theory chapter, there was a strong need to engage in data differently, so the complex phenomenon of leadership was not reduced to fundamental factors already common in the literature. Snyder (2013) states that the exact mechanics of a complex system, however, cannot be recreated due to its inherent resistance to reductionism, making it intractable. Knowing the leadership network to be involved but also knowing the likelihood that reductionism would occur, presented me with a dilemma - how to replicate the system in a way that would allow me to analyse principal leadership practice while keeping complexity intact.

Hallinger (2016) states that educational leadership research needs refining of "current methods and to explore new approaches that enable us to study better how successful leadership responds and adapts to different contexts" (p. 1). This is what Boulton et al. (2015) refer to as "modelling both what happens between tipping points and what happened at tipping points; we are modelling the process of emergence, of evolution, of qualitative change.” (p. 85). Two approaches were used to ensure the impact of reductionism was kept to a minimum and qualitative change could be most evident when representing and describing the network. An overview of these can be found in Table 8 . 


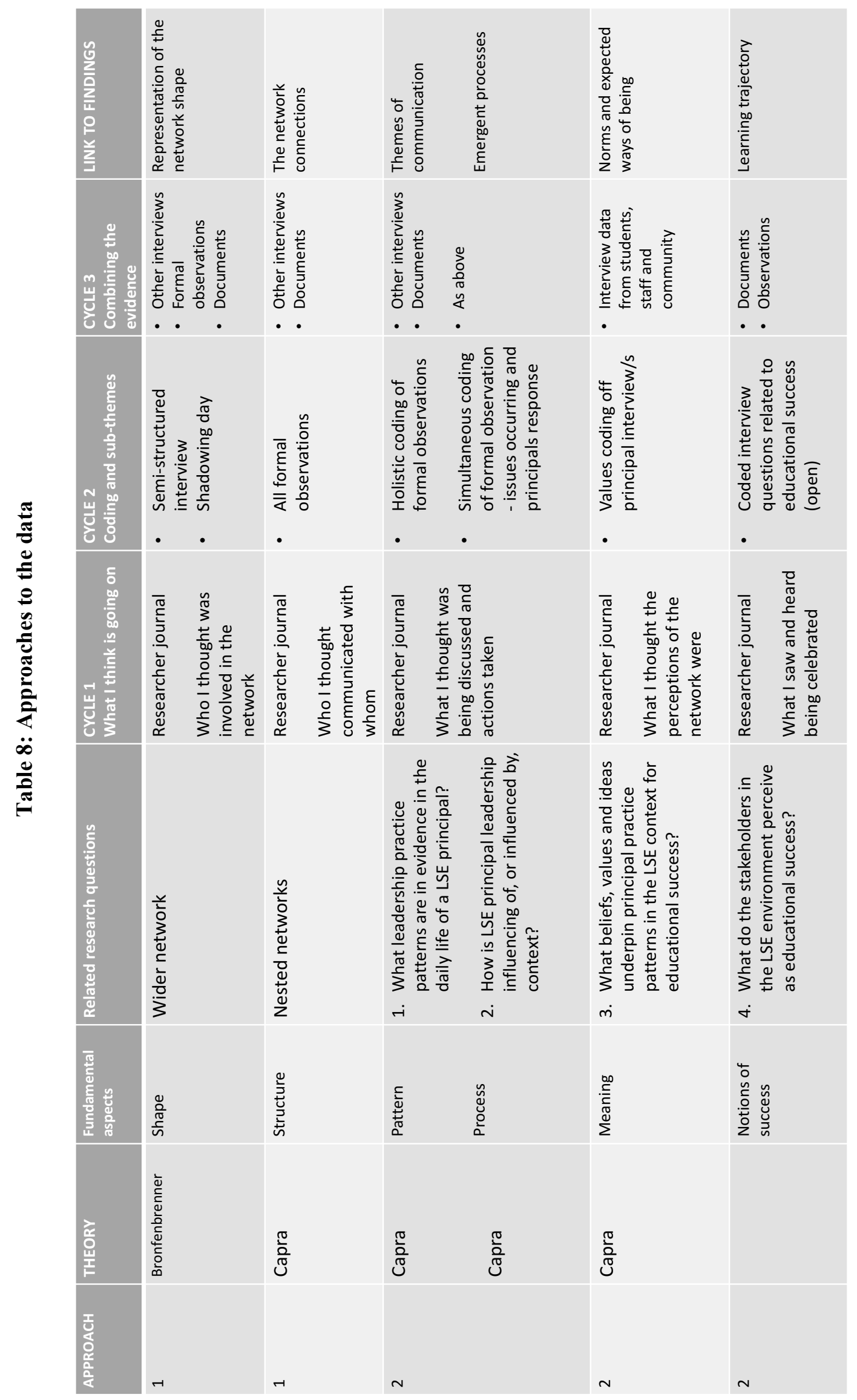




\subsubsection{Representation of the network}

The first analytical approach was intended to help me describe the outer network shape and inner network structural connections of principal leadership practice as a modern phenomenon. The description initially relied on Bronfenbrenner's ecological systems model, but then drew on Capra's living system component 'structure'.

\section{Network shape}

This section of the analysis gave a shape to the network. The shadowing day data were analysed to confirm those previously mentioned by the principal as part of their practice. An adapted version of the Bronfenbrenner model was created for each principal and these diagrams are labelled in the preceding findings chapters as 'outer network'. Broad networks included the community, neighbourhood, and school. Nested networks included: staff, students, aiga/ whānau, MOE and private businesses. Interviews, observations and documents were cross-checked for further evidence. The diversity of the membership of the principal network became apparent, and to some degree, the network boundary.

\section{Network structure}

This section of the analysis gave a connectedness to the network. The first of Capra's (1997) four perspectives of living human systems - that of structure - was addressed.

The interactions across the networks and the nested systems were analysed. Gephi, an electronic open source software was used to analyse and represent all connections noted during formal observations. These were cross-checked with interviews and documents. The groups of people the principal interacted with were all assigned codes. Each code was then assigned connection/s.

Three metrics were used to explain the unique representations: degree centrality, closeness centrality and betweenness centrality. Degree centrality shows the number of direct links each NN has and what connections make up principal leadership practice. Betweenness centrality shows the indirect links between members of the NN. Closeness centrality shows the average length of the shortest path between NN and all other NN in the graph, or who works closest to whom. The resulting 'inner network' diagrams presented at the beginning of each of the findings chapters are a unique representation of Amber's and Cathy's principal leadership. 
The analysis of both the outer and inner structure of principal leadership practice establish the connected and interconnected nature of the network proper.

\subsubsection{Description of the network}

The second analytical approach moved into a more in-depth description of principal leadership practice, by further uncovering themes of communication, emergent processes, norms and expected ways of being; as well as the overall learning trajectory. To achieve this, the remaining components of Capra's scientific understanding of living systems were used.

\section{Pattern \& Process}

This section of the analysis uncovers the continual flow of collective behaviour (Capra, 2016) and the emerging schema of developing understanding, by placing a focus on social constructionism, a theory referred to early on in this chapter. The second and third of Capra's (1997) four perspectives of living human systems - that of pattern and process - were addressed. This analysis generated answers for questions 1 and 2 of this thesis.

First, a holistic code was used to identify topics of communication. I assigned various codes (Miles, Huberman, \& Saldana, 2013) to larger units of observational data rather than line-by-line. Once some initial topics of communication had been established, I looked to confirm and/or find further topics by cross-checking with interviews and documents.

Any tensions or disturbances causing relationships or the school vision and objectives to veer off target emerged as well as any subsequent noticing and adjustments influenced by the principal leadership network. I added in what Miles et al. (2013) refer to as simultaneous coding, a method appropriate when data content suggests multiple meanings. The additional coding uncovered the processes used for the network to self-generate.

\section{Meaning}

The fourth of Capra's (1997) four perspectives of living human systems - that of meaning - was addressed. This analysis generated answers for question 3 and 4 of the study. 
Firstly, it confirmed how the network saw itself. Informal structures give evidence of the growing perceptions of organisational trust and shared norms. A reminder here, that it is the informal structures that hold the most creative potential and learning capability, or what Capra and Luisi (2016) refer to as the state of aliveness in an organisation. I did this by applying values coding on conversations or interview questions that referred to beliefs, values and/or ideas. Values coding allowed me to see how the principal leaders saw their practice and how members of each NN perceived a leader's practice. Miles et al. (2013) argue that values coding is a useful way to ascertain "the way we think and feel about ourselves, another person, a thing or an idea" (p. 67). In the end, various themes emerged that related to meaning/informal structures of principal leadership practice. A commentary on how representatives interpreted practice was only possible when they were also interviewed.

Secondly, the responses to the conversation starters or interview questions related to educational success also held information related to the meaning behind the work of the principal. The answers to such questions were coded using descriptive coding. All ideas were brought together, so that a common, shared purpose of schooling could be derived. According to Miles et al. (2013) descriptive coding provides "an inventory of topics for indexing and categorising" (p. 65). Participants were given the opportunity via the principal, to confirm the final school definition that was created by the researcher.

\subsubsection{Cycles of analysis}

All observations, interviews and documents were analysed using a three-cycle emergent coding process. In cycle one, data were coded during collection in an exploratory way without a specific formula. I wrote into my research journal/field notes what I thought was occurring.

Cycle two related to sub-themes that occurred when I more formally analysed the data. Responses were frequently allocated several codes. I reviewed the coding often to check for accuracy and to rationalise assigned codes. At times, I introduced new codes while still others were merged or were eliminated. Further, I circled, highlighted, and 
underlined what Boyatzis (1998) calls codeable moments - quotes or snippets of data that I thought worthy of attention.

The third cycle of coding involved triangulating observations, interviews and documents for both sub-themes and themes. The identification of sub-themes and themes concluded in this cycle. All three cycles were variously coded and gathered together on a tabular display. A sample copy of a coded transcript (Appendix H) and the tabular display of coded headings (Appendix I) can be found in appendices.

\subsubsection{Data triangulation and cross-case analysis}

Two main methods were used to enhance the trustworthiness and the quality of findings: data triangulation and cross-case analysis. The cross-case analysis further moved the case study into the realm of exploration.

Triangulation was used to see the phenomenon in different ways and for a more indepth or more precise meaning to evolve (Flick, 1992; Patton, 1990). Denzin (2009) identified four necessary forms of data triangulation: data sources, investigative, theoretical, and methodological. Triangulation by data sources was evident in this study as a variety of people and documents were utilised. The principal leader, as well as the staff, students and wider community inclusive of aiga/whānau spoke about their perspectives on the research themes. To strengthen the discoveries made, findings chapters annotate both data sources and data collection methods. 
Table 9: The data collection recording codes used throughout this thesis

\begin{tabular}{|c|c|c|c|}
\hline Code & Data collection methods & Code & Data collection methods \\
\hline A & Acacia School & CSSI & Community semi-structured interview \\
\hline C & Cedar School & C1SSI & $\begin{array}{l}\text { Community one-on-one semi-structured } \\
\text { interview }\end{array}$ \\
\hline ISSI & In-depth semi-structured interview & $\mathbf{0 2}$ & $\begin{array}{l}\text { Direct observation } 2 \text { - leadership team } \\
\text { meeting }\end{array}$ \\
\hline FU & follow-up meetings (1-6) & $\mathbf{0 3}$ & $\begin{array}{l}\text { Direct observation } 3 \text { - governance } \\
\text { meeting }\end{array}$ \\
\hline SA & Student activity & RJ & Indirect observations - researcher journal \\
\hline 01 & Direct observation 1-time diary & DSC & Document school charter \\
\hline SSSI & Staff semi-structured interview & DA & Document appraisal \\
\hline
\end{tabular}

Data sources are outlined at the beginning of each of the findings chapters, while Table 9 above gives the codes for all data methods used. Attaching direct quotes to the evidence discussed in the proceeding findings chapters would help to strengthen validity.

Triangulation relied heavily on researcher interpretation and was strengthened through the services of my supervisor/s. Additionally, I sought out challenging research mentors. One mentor held a $\mathrm{PhD}$ and was a principal, while the other was a chief researcher for an education research and development organisation. Discussions were centred mainly on aspects of the theoretical framework, research design and methodology, including data gathering and analysing processes.

Triangulation by theory involves combining different perspectives to interpret a single data set (Flick, 2018). I employed triangulation by theory in three main ways. Firstly, during the literature review process, transdisciplinary research about leadership informed the design and interpretation of study findings. Secondly, a constructivist and transformational approach helped to evolve a system of relatability. Thirdly, employment of a theoretical framework, was used to influence a mixture of cognitive, biological, social, educational and ecological domains, previously described in detail in the Chapter Three. Methodological triangulation is the use of multiple methods to study a single problem. In this study, various interview types were used such as indepth or semi-structured interviews and observations that were both direct and informal. Students used a unique method, so data were as authentic as possible. 
Then, cross-case analysis brought the various descriptive elements of the embedded case study together to start to explore what could be seen. Knowledge was mobilised (Khan \& VanWynsberghe, 2008) for both similarities and differences between Amber at Acacia School, and Cathy at Cedar School. The purpose of the cross-case analysis was to assist new knowledge to emerge. It should be noted however, that the small sample size precludes an assumption that findings can be applied to wider populations.

\subsection{Ethics}

The importance of ethical research to ensure research environments of trust and respect for both the researched and the research findings cannot be understated.

I sought and was given Victoria University of Wellington, ethics approval. From the moment the study process commenced, I worked hard to ensure my actions aligned with my ethical responsibilities. For example, I allowed principal participants time to decide on whether they would participate in the research without undue influence. Time and space allowed them to gauge the interest of their staff, to decide whether the research would be beneficial to the school overall, and to seek permission from the BOT. Upon agreement from the principal, a letter (Appendix J) and consent form were sent to the school (Appendix K). The research did not begin until these were both received.

All other school community research participants were given time to decide about their participation without influence. Participants in observations, for example, were told about the research and its purpose before any observations took place. Information sheets (Appendix L) and written consents (Appendix M) were provided by the researcher only when participants signalled a keen interest in being involved or, were it had been established they were likely to be present at a formal observation (Appendix N). All were adapted to suit each of the participant groups and samples are included in the appendices.

The study was designed in a way that participant data (Lyman, Ashby, \& Tripses, 2005) were gathered as part of business as usual. Formal observations were of activities already scheduled, and interviews were worked into pre-existing structures such as staff meetings and home-school meetings. 
As involvement in this research could not be anonymous, ensuring participant confidentiality was guaranteed in three main ways. Firstly, I ensured information was secure during processing and analysis. Hard data were kept in a locked cupboard and data held electronically were unable to be accessed without a code. A database was created to house all study notes. Secondly, participant identities were not revealed. The principal was given an alias for ease of story-telling, while all other network member representatives had codes. Thirdly, assurance was sought from all participants that they would respect the confidentiality of all members of the group and would not share any interview discussions with others.

\subsection{Trustworthiness}

To ensure that research is constructed not only of sound procedures but also beneficial aims and results, four characteristics of trustworthiness were in evidence throughout the research process. This section explains how the study was designed and undertaken to address the four principles of trustworthiness: credibility, transferability, dependability, and confirmability (Guba \& Lincoln, 2005).

\subsubsection{Credibility}

Credibility seeks to link the study findings with reality to demonstrate the research 'truth' (Patton, 1990). The interview process supports the credibility of this study. Both adult and student interview sessions ended with a shared consensus on the summarised thoughts and ideas of the session. Transcripts of the conversations were shared, and summary sheets made available on request.

The study also involved members checks (Cohen et al., 2017). Regular follow-up discussions with all adults involved were held face to face or by email. The discussions were useful for coming to a shared agreement about what was emerging. I made myself available to explore ideas and allow for unexpected topics to emerge. Students were given the opportunity to follow up with their teacher and any comments, queries or concerns were passed on to the researcher. While most follow-up discussions were unscheduled, principal follow-up discussions were scheduled, recorded in summary note form, and shared with the principal who checked for accuracy. The discussions occurred after the in-depth interview and all observations, or when otherwise requested by the principal. As often as possible, no more than a week elapsed between the indepth interview, observations and follow-up discussions. 


\subsubsection{Transferability}

Transferability refers to the degree to which the results of qualitative research can be transferred to other contexts (Guba \& Lincoln, 2005). The drawback of a case study is that it focuses on such a unique context and phenomenon making transferability problematic. While this study focused on principal leadership in the LSE context, it is hoped that lessons may be learnt that have more extensive leadership and contextual applicability. The outline of the context and participant characteristics described in section 4.7.1 of this chapter provide a description that will help any reader to compare the relevance of the findings to their setting and hold the reader responsible for making the judgment of how to transfer findings to their context.

\subsubsection{Dependability}

The idea of dependability highlights the need for the researcher to account for the everchanging context within which research occurs (Guba \& Lincoln, 2005). The description and exploration in the discussion chapters attempt to show principal leadership in practice but can only ever be representative since networks can never be fully known or seen (Boulton et al., 2015). An explanation of my theoretical positioning, role in the research and my evaluation of the study limitations conclude the chapter. All aspects facilitate the reader's ability to make decisions about the dependability of results.

\subsubsection{Confirmability}

Confirmability is the fourth principle of trustworthiness and refers to the degree to which the results could be confirmed or corroborated by others. Confirmability checks that participants, not the researcher, shape the findings. Reflexivity and a chain of evidence were used to ensure confirmability in the research.

Mills et al. (2010) suggest that an awareness of reflexivity is vital and entails the researcher being conscious of their influence on the process and outcomes of research. As the researcher, my review of the literature, as well as prior experiences as a student in an LSE setting and later a teacher and school leader, influenced how I presented the research. My ideas about principal leadership practice in an LSE setting and what enhances the success and wellbeing of students in poverty are therefore essential to disclose. Noted immediately after the completion of the research proposal and prior to 
participant recruitment was a list of my prior assumptions which included that principals would likely:

- exhibit the effective leadership practices spoken of in the literature

- be focused on student learning

- distribute and share practices

- acknowledge that poverty matters

- $\quad$ know their students and families

- show leadership beyond school walls

- $\quad$ accept leadership as complex

- $\quad$ spend time advocating against social injustices

- work towards inclusivity

- view educational success in varied ways.

These assumptions gave me some idea of the types of things I might be more inclined to notice as I embarked on the research. Capra (2016) states what we notice depends on who we are and our current state of mind. I worked to stand at enough distance, to be able to see patterns and trends of various sorts but at the time of data gathering, acknowledge that my sensory range included noticing: research-based practices (Parrett \& Budge, 2012), collaborative and distributed leadership (Diamond \& Spillane, 2016), social injustices (Shields, 2013), ethical actions (Kramer et al., 2014), and inclusionary goals and practices (Cherkowski, 2010).

Finally, as advocated by Yin (2014), a chain of evidence was used to keep track of the sources of evidence along with a case study database which was created on my computer and included relevant documents and structural arrangements such as university policies, guidelines, and ethical requirements. I maintained a systemic way of recording data. For example, pieces of data were dated and "time and place of collection" noted (Briggs et al., 2012, p. 165). Back-up files were also kept.

\subsection{Arranging the findings}

The resulting findings are presented using a narrative description (Miles et al., 2013). The description composition: describes the principal leader and their beliefs and values; gives a background description of the school, including the community's 
notions of educational success. Network by network, and nested network by nested network, patterns of principal leadership practice discoverable across the network are additionally explored for who was involved; what was discussed; how patterns of practice evolved including vulnerabilities and adjustments; and the perceived why behind the leadership patterns.

Applying the idea of how a living system self-regulates in nature, meant I would also be able to include nearer the end of each chapter, a comment on the capability of the principal practice network overall. Both cyclical and developmental feedback loops would help to identify whether the network had the ability to sustain itself. The following expectations arose from my theoretical framework:

- both cyclical and developmental feedback loop would be evident if the network was progressing

- if more cyclical feedback loops were in evidence this would largely maintain status quo

- where there is greater evidence of developmental feedback loops, there is more likelihood of emergence

- where something new occurred unexpectedly, this will be evidence of emergence.

- if both types of feedback loops were not in evidence the network may be showing regression

The change trajectory of each network would conclude each of the findings chapters to decide whether the network was capable of 'progressing' (primarily maintaining status quo) or 'transforming' (maintaining status quo while also developing new structures with the possibility of emergence). Moreover, if both types of feedback loops were not in evidence, the network may be showing signs of 'regressing'.

\subsection{Chapter summary}

This chapter has described an embedded, emergent case study which organically develops a rich narrative, highlighting the complex nature of the phenomenon of principal leadership. Participants (inclusive of the researcher), data sources, and methods have all been described, as well as steps that were taken to gather, analyse 
and present findings. The chapter further outlines the process for data triangulation and cross case analysis, which will attempt to identify the correlation between the variables and why the phenomenon develops as it does. This chapter further outlines the ethics and trustworthiness of the study, ensuring that socially-constructed knowledge for improved understanding of the ecology of leadership is credible. 


\section{CHAPTER 5 \\ Principal leadership practice at Acacia School}

“Doing the right thing" Principal Amber

\subsection{Chapter overview}

This first findings chapter describes Amber's principal leadership practice in interaction with the Acacia learning community. The chapter begins with a narrative of Amber's early career, leadership journey, and personal philosophy. Next is a description of Amber's arrival at Acacia School and community notions of educational success. I then explore in detail the eight NN that make up Principal Amber's leadership. The connections that occur across these NN from within the localised LSE setting show the layers of complexity. In the main body of the chapter the composition and patterns of communication detail who is in the network and what is discussed; any disturbances and adjustments are also described to show how the NN move and re-organise themselves. Where possible, perspectives related to meaning are included. The change trajectory is inferred. The chapter concludes by summarising Amber's principal leadership as a collective and individual construct and the likely contribution it is making to wider education reform.

\subsection{Abbreviations}

The following abbreviations were assigned to the Acacia School research participants.

Table 10: Acacia School abbreviations of data sources assigned

\begin{tabular}{|c|c|c|c|c|c|}
\hline Code & $\begin{array}{l}\text { Data sources at } \\
\text { Acacia School }\end{array}$ & Code & $\begin{array}{l}\text { Data sources at } \\
\text { Acacia School }\end{array}$ & Code & $\begin{array}{l}\text { Data sources at } \\
\text { Acacia School }\end{array}$ \\
\hline PA & Principal Amber & & & & \\
\hline ASG & $\begin{array}{l}\text { Acacia staff } \\
\text { group }\end{array}$ & ACG & Acacia community group & AD & $\begin{array}{l}\text { Acacia School } \\
\text { document }\end{array}$ \\
\hline ASA & Acacia staff $A$ & ACA & $\begin{array}{l}\text { Acacia community member } \\
\text { A }\end{array}$ & $A D, S C$ & $\begin{array}{l}\text { Acacia document } \\
\text { school charter }\end{array}$ \\
\hline ASB & Acacia staff B & ACB & $\begin{array}{l}\text { Acacia community member } \\
\text { B }\end{array}$ & AD,TA & $\begin{array}{l}\text { Acacia document- } \\
\text { teacher appraisal }\end{array}$ \\
\hline ASC & Acacia staff C & ACC & $\begin{array}{l}\text { Acacia community member } \\
\text { C }\end{array}$ & $A D, B P$ & $\begin{array}{l}\text { Acacia document- } \\
\text { behaviour policy }\end{array}$ \\
\hline ASD & Acacia staff D & ACD & $\begin{array}{l}\text { Acacia community member } \\
\text { D }\end{array}$ & & \\
\hline ASE & Acacia staff E & ACE & $\begin{array}{l}\text { Acacia community member } \\
\mathrm{E}\end{array}$ & AS & $\begin{array}{l}\text { Acacia student } \\
\text { group }\end{array}$ \\
\hline ASF & Acacia staff $F$ & ACF & $\begin{array}{l}\text { Acacia community member } \\
\mathrm{F}\end{array}$ & & \\
\hline
\end{tabular}


These abbreviations can be found throughout this chapter alongside the data collection recording codes developed on page 95, Table 9. Together these codes are evidence of the data used to confirm findings.

\subsection{Amber}

Amber discussed her early career and educational leadership journey and what inspired her practice.

\subsubsection{Early career}

Being a leader was not part of Amber's plan; however, a series of events led her in this direction. After her first permanent teaching role, and taking some time out for motherhood, Amber and her family moved to a provincial LSE town, where she unexpectedly returned to full-time teaching. Her new role introduced her to Māori culture. Amber realised that:

An effective partnership between schools and parents, whānau, and communities can result in better outcomes for students. (PA, AISSI).

Here Amber noted that achieving a goal is more possible when relationships are established between all those who are proactive in influencing a child's learning.

Amber then worked in a special character school for six years. Undertaking this teaching role enabled her to widen her views on the different ways the curriculum could be delivered. Learning began with the skills and prior knowledge that the children brought with them, and it was these that were developed into the curriculum. Amber reported looking for all manner of change in students including attitudinal, motivational and emotional improvements. Curriculum and educational success became broader ideas.

Six years later and with a more developed interpretation of collaboration, curriculum and success, Amber relocated a third time. A semi-rural primary school gave her an unexpected opportunity to be acting principal and this experience had a pivotal effect on her. Amber reported using her acting principal role to: develop her understanding of the art of teaching and learning; work on team-teaching; develop integrated curriculum; develop content knowledge in literacy; and use portfolios for reporting. 
She created supportive, motivating, and measured experiences for teachers and students.

\subsubsection{Amber's philosophy}

The semi-structured interview and follow-up meetings provided a window into Amber's personal and educational philosophy which revealed several key beliefs: strong relationships, diversity and belonging, deep thinking, and being solutionsfocused.

\section{Strong relationships}

Amber spoke of the importance of strong relationships and their contribution to keeping the school community intact in times of difficulty. Her relationships mattered and required ongoing attention:

Relationships are there as the cement to hold everything together. I also trust they can take a few knocks, which is important in a hard school with hard kids. (PA,FU2)

Strong relationships appear to lead to high-trust relationships and act as a vehicle for getting through difficult times. During a follow-up discussion, Amber noted that humour was also considered integral to a robust relationship:

Not taking yourself or external events seriously helps manage stress and seeing humour in situations can produce positive feelings. (PA, FU3)

Amber was primarily concerned with the practice of leadership as a strategy for developing relationships.

\section{Diversity and belonging}

Amber considered diversity and belonging as a vital component of her philosophy. A diverse cultural setting was defined by Amber as:

Where many ethnicities can share what their life is like, what their expectations are for their children and where power and choice around education success are shared. (PA, ISSI)

This approach shows she did not expect conformity and was aware of the power differentials that existed between home and school. Amber recounted the diverse ethnic makeup of Acacia and reported that diversity enriched the school culture:

You've got to have children that feel like they are part of this big family and we talk about a family. We talk about us being a family as much as we talk about us being a school. But you know being in a family means that you belong to something, it means that you are going to be valued. (PA, ISSI) 
A strongly held belief around the value of diversity was encouraging of the value of belonging. Amber nurtured belonging within the school setting, with the aim of connecting diverse individuals to the greater whole.

\section{Deep thinking and solutions-focused}

Amber explained deep thinking as taking time to reflect and question thoughts and actions. It is deep thinking she says that:

Will eventually lead to a very noticeable difference in what a teacher says or does and to real and sustainable change. (PA, ISSI)

Amber recognised the need to deeply explore pedagogical thinking but additionally appeared to make link between being solutions-focused with her depth of thinking. When problems arise, she reported that it was reasonable to voice frustrations or worries but the expectation is always to delve further into issues so moving forward with a solution is possible:

You just go through your day finding solutions because everything has a solution
but you don't think of it in terms of 'I am doing this, so this can be enacted.' It's
just that this is a solution to this problem so we will make this happen. (PA, FU4)

Amber's belief in thinking things through to some depth meant she articulated her philosophy of education as inclusive of seeking solutions for meaningful change that made sense in context.

\subsection{Amber as principal of Acacia School}

Amber eventually moved back to her home town and the role of principal at Acacia School, a Catholic school she attended as a child. Acacia School shared the same site as the parish church and families reported a close link between the two. School buildings were maintained to standard and included: an administration block; a sick bay; staff toilets; and a staffroom/teacher resource room. Classrooms were described in the school pamphlet as modern and, under Amber's leadership, a new library was established. Core Catholic principles underpinned the vision, goals and curriculum and were listed in the school charter as: respect for yourself, respect for others, and respect for your environment. The school vision was to:

Educate and develop the whole child in a supportive and caring special character environment to become confident, connected, actively involved lifelong learners. $(\mathrm{AD}, \mathrm{SC})$ 
The six school goals, below accompanied by six action plans, gave evidence of how Acacia intended to fulfil the school vision. Goals included:

1. Raising student achievement

2. Developing strong learning relationships with families over three years so that they become skilled and confident when supporting their children's learning

3. Providing a safe and healthy physical and emotional environment for learning and teaching for all staff and students

4. Preserving and strengthening the school's Special Character

5. Encouraging community support and ensuring the multicultural aspect of the school is developed in a planned and meaningful way

6. Developing ICT literacies and understandings for staff, students and their families, to develop confident, responsible digital citizens.

The charter ${ }^{22}$ showed evidence of: future-focused teaching and learning; relationship development; establishing key competencies such as thinking, relating to others and participating and contributing; being concerned with environmental care and fiscal responsibilities; and strong ELL learning programmes.

Amber spent much of the first year observing and meeting as many families as possible. Her first year at Acacia started with a visioning exercise to bring the staff together. As Amber made organisational changes, she grew in confidence into her principal role and had clearer ideas about what she was trying to achieve. She stated:

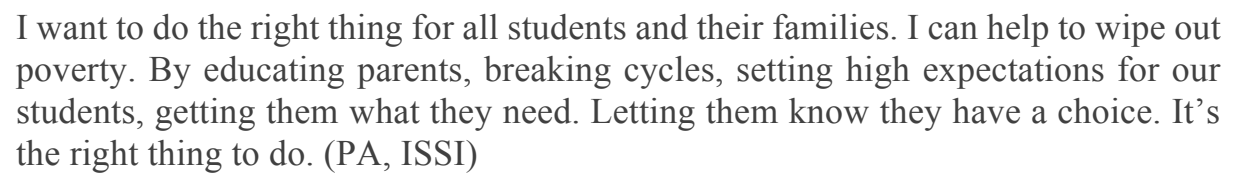
poverty. By educating parents, breaking cycles, setting high expectations for our students, getting them what they need. Letting them know they have a choice. It's the right thing to do. (PA, ISSI)

Here, Amber appears to be working to juggle context, relationships and expectations. Indeed, she likened her principal role at Acacia to that of an octopus (Figure 4).

22 Each school in NZ develops their own charter which is made up of three main sections. Charters include 3 main sections: the introductory section (including mission, vision and values), the strategic section (broad aims covering the next 3-5 years) and the annual section (including specific targets and planned actions) (MOE, 2018). 


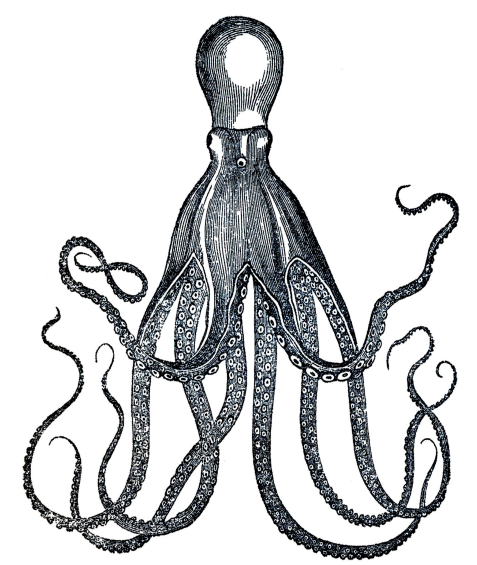

Figure 4: How Amber described her work as principal leader at Acacia School

Amber saw herself as working on many aspects of teaching and learning all at once. She often felt that she was rushed off her feet and never had enough time in a day to complete her various roles and responsibilities.

\subsection{Educational success in the Acacia community}

The Acacia learning community were asked how they defined educational success. Contributors included: staff including of Amber, students, the community including of parents, and documentation including the school charter and NS results. The researcher identified setting goals and expectations, displaying a positive attitude, and the development of the whole person as the three patterns that determine educational success.

\subsubsection{Setting goals and expectations}

The staff placed an emphasis on learning as a process in areas such as literacy, numeracy and behaviour. There was an expectation that children would:

Notice the journey along the way, what they could change, and wanting to make change happen. (PA, FU1)

Learning as an ongoing cycle of action and thought appeared to be a part of the school's coherent approach to learning engagement. Students commented that showing improvement was important to them but they were not able to express how they might show progress, aside from getting good grades. The community emphasised educational success based on various forms of evidence. Parent 1 argued that progress was simply an uplifting feeling experienced by their children of having done well. 
For most parent participants however, it was about how their children were doing compared to how they were doing previously. Parents noted that achieving goals was co-dependent on:

Knowing when movement is not occurring and all of us acting on it. (ACB, CSSI)

The cohort showed concern for removing anything that threatened progress. In addition, setting goals and expectations was thought to create an intrinsic feeling of success or evidence of a measurable goal. This type of success required engagement, a focus on process, and noticing and removing barriers to forward movement.

\subsubsection{A positive attitude}

The staff, students and community agreed that having a positive manner was important as it denoted a sense of happiness in oneself and was encouraging of others. For staff, a good learning attitude was likely to be reflected in the quality of a student's schoolwork and high engagement levels, with students expressing a good attitude through:

Good behaviour, being a good friend and not a bully and loving one another. (AS, $\mathrm{SA})$

This ability to attain and maintain friendships was singled out as an expectation for all subgroups. The community projected forward the advantages of a good attitude, stating:

A healthy attitude to learning now, will encourage students to continue learning. $(A C F, C S S I)$

Adding value appeared to be about creating future-focused learning attitudes or:

Having kids fulfil dreams and contribute to society in a positive way. (ACE, CSSI)

Quotes such as this, suggest that parents wanted their children to go on learning well after they left Acacia School, showing that they could cope in new situations. For parents, this was about having their children recognise their own worth. Having students believe in their own abilities could play an essential motivational role in aligning their worth alongside others. This aspect of collective success links strongly with Amber's belief in belonging. 


\subsubsection{Development of the whole}

Staff, students and the community agreed on the importance of the development of the whole person. Amber expected that students would excel in a variety of activities including sports, music, spiritual, and academic learning. Staff suggested:

Students need to be able to live and work with diversity. (ASB, SSSI)

This as an example, showed staff valued a focus on students understanding their wider social responsibilities. Amber additionally felt that being able to work alongside others and to know what fair treatment looks like was especially important since social wellbeing might enhance academic learning.

For students, achieving in areas such as sports, music and art endeavours was at the forefront in their minds. Representing the school at regional sports day, swimming meets, arts festivals, being in choir and the school band was, for them, important evidence of success. Students understood that success does not always look the same for everyone. Community member two added:

To have somebody leave the school and they are a good person. They mightn't be an 'A' student but they know a bit about that, and a bit of that, and have found what they love and are good at. You're educating the whole person. (ACA, C1SSI)

At Acacia, the process of finding your niche in life, is identified alongside more traditional notions of success. The development of spirituality was added by a community member who stated:

Connecting with spiritual, gospel dimensions of life, not just interacting on the surface of life. (ACA, C1SSI)

Several people discussed being able to show Catholic values in their everyday life, as evidence of the depth of their development. The Acacia community valued the contribution of schooling to the development of the whole person. The development of a well-rounded individual who is a good person and has found their strength is important to the Acacia learning community. Success is inclusive of a strong spiritual identity.

\subsection{Representing Amber's principal leadership network}

In this section, I represent both the outer and inner forms of the network. The outer network is made up of three smaller networks and those three smaller networks are 
made up of eight NN. The inner form of the network builds up a picture to show how $\mathrm{NN}$ connect and move across the various boundaries.

\subsubsection{The outer network}

The outer network shows three smaller networks that became evident during a 12-hour day, a day Amber described as slow.

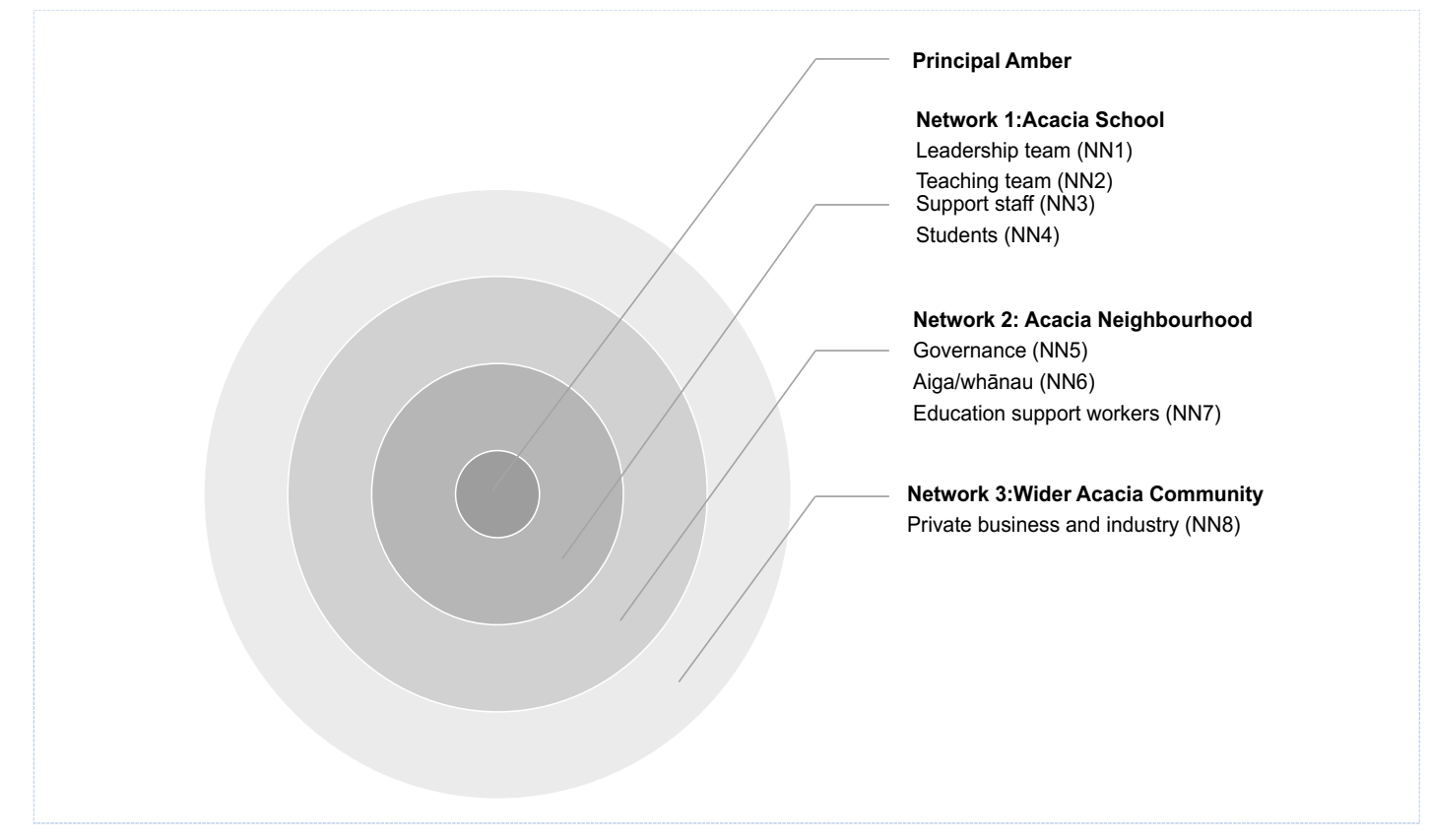

Figure 5: The networks and NN evident in Amber's social relationships across a day

The principal leadership network at Acacia comprised:

- Principal Amber who was a system of her own and included aspects of her upbringing, career and leadership journey

- Network One which included the leadership team, teaching staff, support staff and students. This network was concerned with the quality of day to day learning for students and teachers.

- Network Two which was made up of the BOT, aiga/whānau and education support workers. This network was especially concerned with learner/whānau needs.

- Network Three which included private business and organisations. This network appeared to be helping to sustain a healthy teaching and learning environment and developing resources. 
Figure 5 gives an indication of the groups of people Amber drew into each of the three wider networks of her principal leadership practice. Amber had self-directed boundaries. That is, who she brought into each network of interactions, the number of relationships and connections and who is and is not included were all determined by Amber.

\subsubsection{The inner network}

The inner network describes the connections that took place across the NN in a day. Each $\mathrm{NN}$ was represented in the research by a variety of people, with each group having their own code. Amber was a part of each NN, so this representation is uniquely hers.

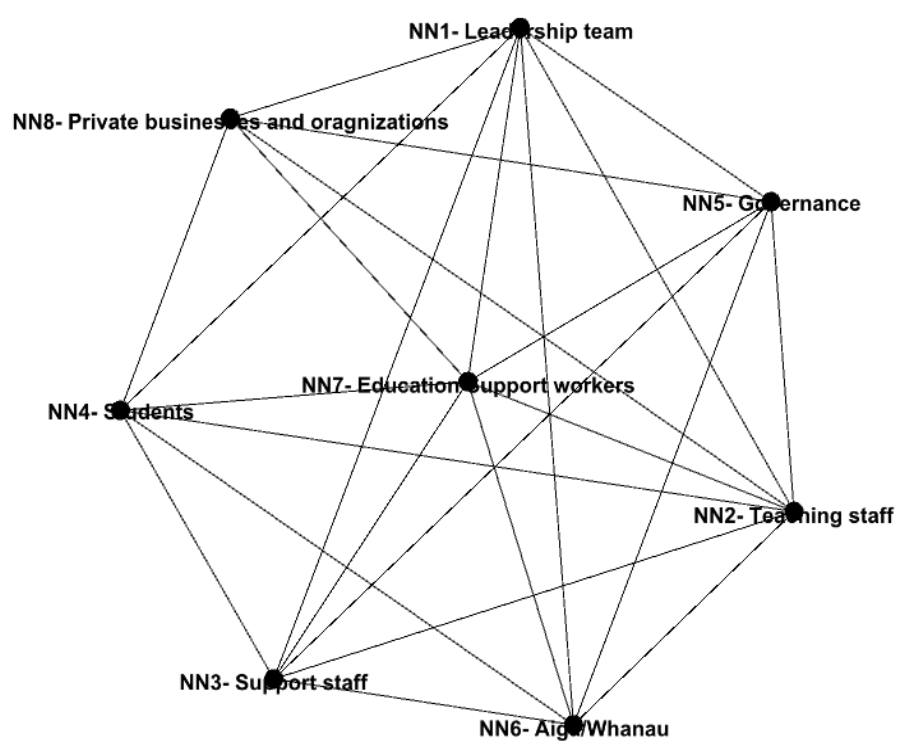

Figure 6: The connections from within Amber's principal leadership network

The following aspects of Principal Amber's inner network, visualised in Figure 6 become clear:

- the network is well connected. While private businesses/organisations have only 5 of a possible 8 connections, all other NN having between 6 and 7

- the teaching staff and leadership team are highly interconnected with 21 and 20 interconnections respectively. Support staff, governance, and aiga/whānau are not far behind with 17,17 and 16 connections

- the least interconnected are students and private business and organisations 
- the NN participants did not necessarily connect with each other. Amber worked with individuals within two out of the eight $\mathrm{NN}$ to support specific needs and interests

- the distance between the connections is evenly spread except for NN7 which is marginally further from all the others.

Aspects of degree centrality, closeness centrality and betweenness centrality (explained in the methodology chapter, section 4.8.1) all show that the eight $\mathrm{NN}$ are connected, interconnected and on the whole work together for a stable principal leadership network.

\subsection{Describing the network}

The quality of the network is now described, drawing mainly on observations and interviews, but at times, documents. Indicative examples of how each $\mathrm{NN}$ responded to any contextual disturbances and then self-corrected to keep the purpose of the network in focus, is described. Where possible, informal structures are explained from those within the network/s.

\subsubsection{Network One: Acacia School}

Network One is the Acacia School community and included representatives from the leadership team, the teaching team, school support staff, and approximately 95 students. The NN within are numbered $1-4$.

\section{Nested Network 1 - Leadership team}

The purpose of the leadership team was defined by Amber as making school-wide management decisions, conveying vision, and supporting staff for greater student success.

WHO

This recently formed group was made up of three members, with two of the three members of the leadership team also being classroom teachers:

- Teacher one - led the senior school and literacy curriculum area

- Teacher two - led the junior school and numeracy curriculum

- Amber provided oversight of school organisation and encouraged capacity building on others. 
All in NN1 provided data and showed evidence of establishing connections with all other NN.

\section{WHAT}

The main patterns of practice evident in NN1 included engaging aiga/whānau in learning opportunities, developing ICT, and engaging in PLD.

Firstly, engaging aiga/whānau was communicated by Amber and staff as intrinsic to the school. While the charter goals and subsequent action plans were focused on developing reciprocal relationships with families, a lack of parental engagement was an ongoing issue. Amber was aware of this but stated:

\section{Being too heavy handed with parents about issues means losing kids. (PA, FU3)}

This awareness meant Amber sought alternative support so parental unavailability did not have a negative effect on the school roll which was fragile. Lead Teacher One, however, suggested a lack of parental engagement may be a sign of the times:

\section{Both parents are generally working. It was different when you had at least one parent who was at home that could take that added interest in kids and what they were doing at school. You know, I even think of my own children. I feel so guilty because I haven't turned up to this or I haven't been to that but it's just an impossibility to get there. (ASF, SSSI)}

This example shows that there is possibly a lack of engagement in both high and low socio-economic school settings.

Amber responded to a lack of parental engagement by making minor timetable changes but also sought outside support to better understand why aiga/whānau may be detached. Amber reported in follow-up discussion five, was that the school had applied to be involved in a MOE initiative entitled the Talanoa Project. This project examined Pasifika culture, language and identity to improve on community engagement, and share ideas about how to together improve student outcomes. Amber hoped the project would:

Revitalise and engage the community in an authentic way that is mindful of contextual restrictions. (PA, FU2)

This appears to suggest that there is a focus on finding more culturally appropriate ways for aiga/whānau to get involved in school. Amber also created her own, in-house solutions, for example, organising a holiday programme at the parents' request. Teacher A reported: 
The parents talked about keeping learning going during the holidays and that they'd like the school open. So, Amber said "ok we'll organise it". She organised people to come in and take weaving and then she had some of us doing a maths thing, and she was doing reading to the kids. The idea was that parents would be involved so there was a (learning) conversation between parents and children. It turned out that the parents just dropped kids off and went. I think the parents need to take more ownership. She felt quite let down that parents had asked for this, they'd made a big thing about it. (ASB, SSSI)

This quote, and those like it, show a mismatch between what the parents viewed as their role and what the school viewed as their role in learning. While the change had the potential to be develop new ways of dealing with school holiday learning, and create new connections, what happened instead was this activity became a one-off. The principal concluded that while school culture was supported by the school charter, a different solution for improving parental engagement levels was required.

Secondly, there was an emphasis on developing ICT needs. The ICT development plan created by Amber and Lead Teacher Two clarified how charter goal six might be met and included a review of computer and internet usage policy, education around safe internet practices, and continuing to build staff expertise.

Resourcing factors created a barrier to developing pedagogical thinking. During Observation Two, the team discussed the new Bring Your Own Device policy. Lead Teacher Two noted that lockable cupboards in which to secure devices were unavailable to most teachers. For students without personal devices, school computers would be available. A form would be devised by the leadership team to allow for ICT stocktaking at a classroom level. Most computers were reported as working adequately while the school server was:

On its last legs. (PA, FU2)

This comment shows that integrating and embedding ICT was affected by unreliable resources. Amber hinted that limited pedagogical understanding by teachers also held up development in this area.

Thirdly, communication was focused around developing both teacher and whole staff learning. Professional learning programmes and expertise were in evidence and included teacher inquiries, Practice Analysis Conversations ${ }^{23}$ and monitoring

23 Practice analysis conversation are an improvement-focused feedback discussion built around teacher observation. 
meetings. An associated structure, the staffing appraisal process, outlined what PLD looked like for individual teachers. The appraisal process was overseen by a member of the senior leadership team and written up as a 'mandatory requirement,' with links to the registered teacher criteria. Staff PLD in conjunction with the appraisal document helped to record the staff's collective and individual teacher responses to school goals.

I noted that before $9 \mathrm{am}$ or after $3 \mathrm{pm}$ were the times Amber could be directly involved in PLD activities with teachers. During the school day, the small school roll rendered Amber unavailable for direct involvement in teacher-learning activities due to her classroom teacher release commitment:

A conflict exists between being lead learner and managing the school so everyone is clear to learn. (PA, FU2)

Amber's lack of availability meant that most PLD was dealt with by experts who guided teacher learning, or literacy/ numeracy lead teachers who took on the lead learner role:

Supporting others to build their own capacity is part of my role too. A certain amount of delegation and trust happens since you are not necessarily directly involved. (PA, FU2)

In follow-up meeting three, Amber commented that if time was available to be involved she would spend it developing ideas with staff and getting more involved in pedagogical development. This is consistent with her desire to think deeply to ensure real change. Between building capacity in other leaders and budget constraints, Amber was precluded from having much direct involvement in teacher learning.

How

Practice patterns were vulnerable when:

1. there was minimal reciprocity from aiga/whānau

2. ICT resourcing held up pedagogical development

3. budgetary constraints saw PLD and appraisal structures as difficult to coordinate in order that Amber could be included in learning conversations.

The adjustments made in NN1 were as follows:

1. NN1 discussed the difficulties families had in supporting their children's learning and made ongoing minor adaptations to timetables and documents. Additionally, new initiatives were trialled 
2. Amber noticed the ongoing lack of improvements made regarding parental engagement and took on an external opportunity as an alternative way of appealing to the community

3. a stocktake and external grant might allow for initial ICT resource barriers to be addressed

4. Amber helped to address the funding challenge by showing flexibility with the principal role. Often, she went into classrooms to teach so other staff could attend PLD with outside experts.

Principal leadership in NN1 made minor changes to the school structures but also recognised the bigger picture reasons for a lack of parental engagement and worked on a longer-term solution to empower the local community in a culturally appropriate way, addressing resourcing issues by any means possible and showing some flexibility with the principal role.

\section{Nested Network 2 - Teaching staff}

Amber reported the purpose of NN2 was managing the teaching and learning needs of classes, developing and sharing their practice and contributing positively to the culture of the school.

WHO

NN2 had 11 people with an average tenure at Acacia of 16 years, and each person carried responsibilities, which were distributed according to strengths and interests:

- five full-time classroom teachers (including a teacher in charge of community liaison, sports and learning support)

- a part-time ELL teacher

- $\quad$ up to four teacher-aides

- Amber who taught regularly.

All members of the teaching staff, other than the teacher aides, provided data about NN2 and showed evidence of establishing connections with all other NN.

WHAT

The main patterns of practice evident in NN2 included supporting the resourcing of family health and wellbeing needs, helping students manage their behaviours, and 
engaging students in a comprehensive list of activities to help them find purpose and connectedness to their learning.

Firstly, resourcing of family health and wellbeing needs was of concern. A pastoral care policy outlined care for students, staff, BOT, families and the members of the school community. The Pastoral Care Policy which sits within the charter assured that:

\section{No child shall miss out or be disadvantaged in any way due to financial hardship or family circumstances. $(\mathrm{CD}, \mathrm{SC})$}

The policy had a budget which paid for lunches, uniforms, stationery and class trips. However, informal discussions revealed there was a mismatch between how staff and Amber believed this policy should operate. During the staff interviews, Teachers One and Five suggested that aspects of the pastoral care policy created a reliance on the school:

With parents at work, we take kids to doctor appointments. By doing this am I enabling the parent to step away from parenting? It's hard because you don't want the child to suffer. (ASB, SSSI)

This phrase is consistent with the previous idea that engaging parents was difficult. Some staff believed parents needed to reclaim responsibility for ensuring their own children's wellbeing. However, Amber stated:

A lack of availability is in no way a sign they do not care. I don't believe that for a minute... but perhaps it's a direct impact of personal circumstances surrounding employment and health. (PA, FU4)

Here, Amber saw parents as impacted by their circumstances and made staff more aware of these pressures. For example, during a morning staff catch-up session, Amber touched on the many requests made of aiga/whānau which just that week included the payment of school fees, grocery items for the school fair, and transporting students to local sports events.

Secondly, students were helped to manage their behaviours. A behaviour policy aimed to:

Collect and use data about high behaviour needs, devise and implement a whole school reward system and work to incorporate restorative practices throughout the school. (AD, BP)

All teachers had responsibilities associated with this plan and timeframes were included. Staff reported discussing problems and ideas with Amber, noting that Amber would suggest strategies or assist them directly. More complex behavioural issues 
noted by staff members one, three and four required Amber to visit students' homes and talk to parents. Any changes in student circumstances were fed back to individual teachers:

Amber grew up in the area so she is part of the place. She visits the home. She's not a threat. (ASB, SSSI)

A number of teachers discussed Amber's access to and understanding of aiga/whānau social contexts enabled her to deal with the various dynamics that may be affecting learning in school.

Thirdly, engaging students in a rich curriculum was considered. Learning was focused on: Christian values such as respect for self and others, key competencies such as thinking, relating to others and participating and contributing, sustainability, environmental care, and fiscal responsibility. The breadth of the curriculum contributed both positively and negatively to the school culture. Staff acknowledged that Amber's ability to bring sections of the community in, for example, to volunteer for breakfast and reading clubs, support secondary school students for homework club and tutor for violin club, were all worthwhile. However, a staff member stated:

It feels like a community centre sometimes, when there is nowhere to work. We are over-committed. (ASB, SSSI)

Although areas of literacy and numeracy were prioritised and additional activities welcomed, the opportunities also created timetable pressures for teaching staff.

Amber reported in follow-up meeting three that she constantly evaluated the programmes offered so as not to overcrowd the physical space and timetable, but she also maintained that the school was central to the sustainability of the community:

\footnotetext{
We are a community. If I had my way, and this causes a little bit of conflict, it would be even more so. We would have the school open for an awful lot of hours. We have things like violin after school, we have had cooking after school, we've had all sorts of things - sports afternoons where the local cricket club come down. I quite like having the community using the school. We did computers in homes last year and that got 16 families a computer, so to me it is, you know, it's the centre of the community certainly. (PA, FU6).
}

Amber's approach meant that new opportunities for students and their aiga/whānau would at times emerge unexpectedly, while teachers preferred the school day to be more planned. Developing such opportunities possibly helped to develop the whole child, an indicator of education success referred to in Section 5.4. 
How

Practice patterns were vulnerable when:

1. there were varied assumptions held by staff about what parents could/should remain responsible for

2. student behaviour issues were not easily reconciled

3. learning opportunities were perceived as detrimental to time spent on the formal curriculum.

The adjustments made in NN2 were as follows:

1. Amber recognised and responded to welfare requests by families, ignoring more negative teacher perceptions. Amber also ignored the unsuccessful attempts at developing home-school partnerships and worked instead to re-focus expectations

2. Amber continued to work one-on-one with aiga/whānau to create a collaborative approach to behavioural issues. Extreme behaviours required a careful balance between helping children to take responsibility for their behaviours, teaching children different ways of behaving, supporting teachers, and assisting with family welfare needs

3. Amber timetabled additional opportunities for students that were related to school goals and proactively worked to balance current learning with future opportunities.

Principal leadership in NN2 challenged teachers' perceptions of their respective roles in the school and community, acted as a link between aiga/whānau and teachers, and opened the curriculum up to more holistic teaching and learning opportunities.

WHY

NN1 and NN2 representatives perceived the meaning behind their interactions with Amber as being about building a team, showing inclusivity, and being positive.

Firstly, staff viewed Amber as part of a team, hands-on and visible. The range of activities that Amber involved herself in were noted in the researcher's diary as: school trips, testing, duty, and taking students to appointments. Also noted were the words staff used to describe her involvement and these included friendly, inviting, and welcoming. 
Secondly, staff agreed that Amber was inclusive. This was apparent, they said, in the way she related to families and students:

She made a point of having a conversation with students and families; just connecting with them briefly. They can ask for anything. (ASB, SSSI)

This quote is indicative of the views of many and how Amber welcomed students, families and the community as 'partners in learning' where any barriers to success could be more collaboratively mitigated.

Thirdly, Amber was perceived as a positive influence on the learning community:

She always looks at things in a positive light. It doesn't matter what it is, it doesn't matter who it is, she will always look for the positive first. (ASC, SSSI)

This is one example of how she was seen to enact a positive frame of mind so solutions could be found. The attributes of inclusivity and positivity noticed by others in NN2, are consistent with Amber's philosophy described earlier.

\section{Nested Network 3 - Support staff}

The support staff worked directly and indirectly with Amber on a range of school administrative and property tasks that were focused around workplace support.

WHO

The membership of NN included:

- secretary

- office assistant

- caretaker

- cleaner.

Four support staff participated in the research, three of whom were also parents/past parents. All members of the school support staff team provided data about NN3 and showed evidence of establishing connections with NN1, NN2, NN4, NN5, NN6 and NN7.

WHAT

The main patterns of practice evident in NN3 included creating a safe and orderly environment and aiga/whānau wellbeing. 
Firstly, safety and orderliness were evident across the site. A school health and safety policy was driven by National Administrative Guideline. ${ }^{24}$ The school policy aimed to:

Provide a safe and clean environment ... with safe methods and practices ... preserving the long-term health of the community ... showing care for self, others and the environment ... (AD, SC)

Thirty procedural statements around health and safety supported the policy and these could be found in a booklet entitled, "The Emergency Response Guide." The guide aimed to outline ways of keeping people in the school physically and emotionally safe, especially during unexpected occurrences, and provided guidance on a civil defence plan, a lock-down plan, and chemical emergencies.

Support staff member one mentioned physical safety as an ongoing issue. It is understood that Amber directly contributed to a safer and tidier school environment. People littering and smoking on site were asked to move on and a motorcyclist speeding through school grounds during school hours was also commented on as eliciting Amber's action:

She's gone and put a rope out there so he doesn't come through and could possibly harm one of the students, just making sure that the kids are safe in that aspect. (ACC, CSSI)

This example suggests that when at school, students can be assured all precautions are taken to ensure their safety. Amber also mentioned, in follow-up meeting two that the school neighbours were helpful in guarding school property out of school hours. Much was done in support of goal three of the school charter which is related to the safety and health of students.

Secondly, ensuring the wellbeing of support staff was discussed. Personal wellbeing of support staff was covered by a national collective employment agreement which included PLD, remuneration, expenses, allowances and leave. During interviews, support staff members one and two reported that Amber regularly communicated with them about working conditions, verifying all hours worked were compensated for, and

24 National Administrative Guidelines set out the administrative requirements of the BOT. Guideline 5 states that the governance of the school is required to comply, in full, with legislation currently in force or that may be developed, to ensure the safety of students and employees. 
any annual increments received. Additionally, Amber would accommodate their need to take leave for tangi ${ }^{25}$ :

She volunteers us for things, she must learn to say no. (ASC, SSSI)

This quote suggests that the wellbeing of NN3 required others to be prepared to share the workload more widely, which was not always welcomed. This shows a threat to what Amber previously mentioned as a vital component of her leadership - the nurturing of relationships.

How

Practice patterns were vulnerable when:

1. there were ongoing security and safety concerns

2. the expectation that cultural needs of NN3 would be met by others in the wider network.

The adjustments made in NN3 were as follows:

1. Amber personally took on securing the learning environment during school hours. Out of school hours she called on school neighbours

2. Amber continued to support staff/parents to attend important cultural gatherings as they arose.

Amber's principal leadership across NN3 secured an environment for learning and set an example for how the wider network would respond to aiga/whānau cultural needs in the future.

\section{WHY}

NN3 representatives perceived the meaning behind the interactions with Amber as being about showing inclusivity. Firstly, Support Worker One stated that Amber actively embraced different perspectives:

She dressed up that night in a puletasi, ${ }^{26}$ when she greeted her International Service Organisation friends. Parents said to her, you know, this is a privilege to do this for you (provide dinner) and she said that seemed ridiculous because for her it was a privilege that they were doing it. (CC, CFG1)

This quote shows Amber was involved and encouraging of bringing different facets of the community together and these further evidenced her philosophy in action.

25 Māori word for funeral.

26 A puletasi is a traditional item of clothing worn by woman in the Pacific. 
Secondly, support staff noted that Amber treated them inclusively or "like family" and her door was reported as always open to new ideas:

There was never a difference between if you were the cleaner or whether you were the principal, you always were a part of the school. If we have a social event or anything, everybody is invited. (ACD, CSSI)

Here the suggestion was that Amber's participation was genuine and not attached to hierarchy or power.

Support staff spoke of the way Amber was enhancing the school's reputation and her place in the wider community, recognising that establishing the school's place as part of a broader network was essential. Amber was reported as getting personally involved in activities such as the local community garden and play centre groups:

She cares deeply about the community and shows great pride in school, bringing the two together where she can. (ACA, C1SSI)

Many support staff expressed Amber's belief in the value of diversity and belonging as laying the foundation for the success of students and their aiga/whānau in their own communities.

\section{Nested Network 4 - Students}

A student's main task at school was referred to by Amber as building cognitive, social, physical, emotional, and cultural skills.

WHO

There were five classes that covered Year One through to Year Eight. There was also an ESL class catering for children at all levels but with no permanent roll. A class of Year Seven and Year Eight students (11 boys and 14 girls), as well as Amber, provided data about NN4.

The student group showed evidence of establishing connections with NN1, NN2, NN3, NN6, NN7 and NN8.

WHAT

The main patterns of practice in NN4 included a positive learning culture and understanding success as a process.

Firstly, the network communicated about a positive school culture. Using the Positive Behaviour for Learning Programme alongside the core Christian values, the behaviour 
framework focused on changing the environment, systems and practices to support students to make good choices. Students reported being clear about expectations. Many of their conversations with Amber were behaviour based:

She helps us sort out fights or arguments and discusses consequences. (AS, SA)

While Amber mediated between students, the students themselves informally applied behaviour expectations upon one another. BOT Member Three referred to the following incident:

\begin{abstract}
An older student in Year 8, he goes over and he holds this attacking young fella, calmed him down, spoke to him about respect and later he informs the staff. To me, that spoke volumes about what sort of an atmosphere and attitudes have been nurtured. (ACA, C1SSI)
\end{abstract}

Students assisted staff to curb inappropriate behaviour so the school playground was safe and this likely, in turn, improved the school culture. This is an indication of how the whole child was being developed.

Secondly, the process of learning was linked to the process of success. Students reported being involved in conversations in relation to not just behaviour but also their academic work and health. They viewed these conversations as encouraging and as a good opportunity to set goals and discuss where they might improve, with Amber making what they described as:

Good and sometimes negative comments. (AS, SA)

Portfolios were also used to assist students to explain their learning over time and across disciplines. Amber explains the portfolio:
A portfolio can show an awful lot of progress. You know the Junior School they do a lot of photographs so they have discovery time or they might have a PE session and so there are photographs there capturing what their children are doing. It just makes it real I think. (PA, FU1)

Amber reported that portfolios not only helped students to understand their strengths and next steps but also helped them connect with parents about their learning journey, especially those with ELL. The articulation of learning as a journey is an example of Amber's beliefs on deep thinking.

How

Practice patterns were vulnerable when:

1. ongoing behavioural issues occurred

2. parents had difficulty engaging in their child's learning journey. 
The adjustments made in NN4 were as follows:

1. the adding of core Christian principles and restorative justice to the school behaviour programme appeared to give students a way of managing old behaviours and learning new ones. Student leadership behaviours emerged unexpectedly

2. Amber encouraged the use of portfolios to help narrow the gap between what is taught and what parents know about their child's learning.

Principal leadership in NN4 taught behaviours to improve the learning culture and helped widen the understandings and evidence used to show parents what their child's educational success looked like.

WHY

NN4 representatives perceived the meaning behind interactions with Amber as being about being given good role-modelling and fostering belonging.

Firstly, Amber was perceived as the type of person they wished to emulate. Students stated that she made a great teacher as well as a great leader:

She's caring and positive. Calm and generous with time, as well as hardworking, independent, strong willed, brave and courageous. (AS, SA)

This comment suggests Amber was perceived to be someone whose leadership exuded gentle determination.

Secondly, students emphasised Amber's ability to foster a feeling of belonging. Students described their school as being like a 'second family':

School is a home away from home or a second family. (AS, SA)

A number of people highlighted how Amber's philosophy is consistent with her belief about strong relationships.

\subsubsection{Network Two: Acacia neighbourhood}

Network Two comprises NN5-NN7. The frequency of these relationships varied and teaching and learning less directly supported.

\section{Nested Network 5 - Governance}

Amber was involved in the governance group, the BOT, whose main task she reported was to ensure the school is efficiently run, compliant with national guidelines, and that 
teaching and learning is underpinned by sound practice. The governance group was also responsible for ensuring the school promotes and stays within its Catholic character.

WHO

The membership of NN5 included:

- five elected parents

- one teacher representative

- four representatives of the Catholic faith, including the parish priest

- Amber as the school's educational leader and school manager.

At least one member of the group had been seconded and a member reported the difficulty in retaining members for the full three years as often juggling work, childcare and governance responsibilities became impossible. The entire governance group, minus one parent representative, provided data about NN5. The NN showed evidence of establishing connections with NN1, NN2, NN3, NN6, NN7 and NN8.

\section{WHAT}

The main patterns of practice evident in NN5 included teaching and learning, ensuring adequate funding for day to day schooling needs, and balancing inter-school cooperation and competition.

Firstly, measures of success were considered. The Year Seven and Year Eight cohorts were identified as making inadequate progress in numeracy since over half this cohort were below or well below NS. The same cohort was identified as the lowest performing group in reading. While there was concern, the reliability and validity of the NS results were questioned. During Observation Three Amber commented on a perceived flaw where the jump in expectation was too great:

Last year, I think it was 35\% of students across the country in Years Seven and Eight were reported as at standard in Maths. (PA, O3)

Acacia was no exception and as such, goal one of the school charter included working to improve Year Seven and Year Eight maths. Amber stated:

We know our students are successful and we must believe that even if those out in the wider community think differently. We have a much richer picture not evident in NS results. (PA, FU5) 
Here it appears that the narrow reporting measures had a negative effect on public perceptions of the students of Acacia and they worked against the school's aim of having a positive attitude to learning.

Prevailing deficit thinking acted to undermine expectations. BOT Member Five believed that a lack of home support negatively affected in-school success. This board member, also a teacher, identified that more home support would lift reading comprehension. Amber remained positive that teachers could make a difference and reported during follow-up meeting three that she worked to maintain a confidence that the complex context can be negotiated by:

Bringing a focus back to teaching and learning solutions. (PA, FU3)

This is consistent with her pervious quote related to having a positive attitude but together with the previous quote suggests remaining positive is sometimes difficult.

Secondly, ensuring adequate funding for day to day learning needs was explored. The school property and financial reports outlined health and safety, essential infrastructure, modernised learning environments, and forward planning for discretionary projects. Amber reported in follow-up meeting two that, as the BOT was new, she would spend time working alongside them to develop skills. The BOT were also inexperienced in matters of property and finance and this inadequacy was especially evident when the BOT discussed the recurring flooding in the courtyards, ongoing fence repairs and wooden structures rotting due to age.

Meeting the minimum cost required to maintain the school with limited financial proficiency in the BOT was of concern. For example, asphalting the car park required a grant of $\$ 18,000$. In applying for the grant, $1 / 4$ of this amount was needed in the bank and the funds needed to be locally raised. With this money unavailable, various fundraising ideas were discussed including a car boot sale, stocktaking for the local supermarket, and a school gala but all were deemed impractical within the given timeframe. Amber agreed to action the grant with the hope of finding a private donor.

Additionally, Acacia School was re-categorised by the MOE as a decile '2e'. The higher decile ranking signalled a loss of $\$ 23,000$ and added a further strain to the budget. Amber reported in follow-up meeting two that she expected to honour purchases that developed the whole child and: 
Replacing furniture in classrooms, giving the grounds a face lift or adding to our meagre playground is not a priority. (PA, FU6)

The physical environment suffered because of reduced finances. Amber continually questioned the BOT decision about how money was used, which created tensions between political and economic demands (political, as in how strategic decisions impacted on school results and teacher wellbeing; economic, to ensure efficient and timely fiscal prioritisation). Amber described such situations as requiring ethical decision-making and she believed principals in the LSE setting must act according to what is respectful and fair.

The year the research was carried out, the roll at Acacia fluctuated between 95 and 105 , often sitting just below 100 , mainly due to the removal of state housing ${ }^{27}$ from the community. Moving abroad or an inability to pay special character fees were further reasons given by Amber for families leaving the school. With roll numbers down, the school operational budget was also reduced. Budgeting to keep track of the roll fluctuations and guaranteeing there were essential school resources was an ongoing struggle. For example, Amber explained that a full-time ESL teacher was needed but only a part-time specialist could be funded. Amber personally provided teacher release to offset the cost.

Thirdly, a balance was sought between inter-school cooperation and competition. The governance group could relay positive public feedback about the school. BOT Member Three spoke of aiga/whānau who had moved out of the area but wanted her children to continue at Acacia because of the smaller classroom ratios, good teachers and wonderful principal. The staff representative noted:

Someone must hear about us outside of the community. Maybe that's why so many ELL kids are coming to our school. (ASB, O3)

This example shows that the school was viewed as capably led, enabling an effective response to the diversity of students it attracted. This aligns with students' perceptions of their principal.

${ }^{27}$ State housing is a system of public housing in New Zealand, offering cheap rentals to occupants on low incomes. 
Maintaining a positive school image was ongoing however. In Observation Three, the BOT heard from Amber that a neighbouring school was portrayed negatively in the local press regarding roll decline, staffing problems and limited finances. Amber used this example as a lesson:

It really is not a good idea to share those struggles out there in the community. We need to share our successes. (PA, OB3)

The location of the school meant there was ongoing tension between maintaining a consistently positive public image and yet having an awareness of other schools in the local area that were struggling. Maintaining this tension meant competition between schools could be managed but is an indication of Amber having to work in a less than preferred way.

How

Practice patterns were vulnerable when:

1. distorted achievement measures and deficit theorising were used as reasons for underachievement

2. there were limited finances, limited financial expertise and an ever-reducing budget

3. there were negative images of the achievement possibilities of schools in the wider community

4. there was competition between schools.

The adjustments made in NN5 were as follows:

1. Amber recognised when staff reverted to a deficit mentality. She often insisted on an inclusive framing to reinforce learning as the focus and remained upbeat about possibilities

2. there was an increasing need for principal time to be used to find solutions to finances. Amber used a strategic approach to offset constraints. A more sustainable solution relied on a growing roll

3. Amber worked to keep the BOT abreast of the wider issues faced by all principals in the area

4. Amber worked to support schools in the area while showing a willingness to publically acknowledge Acacia School's point of difference. 
Principal leadership in NN5 refocused efforts away from deficit theorising, remained positive about influences of possibility, developed a strategic approach to balancing the school budget and, while working to widen the COL network, also celebrated that which made Acacia different.

WHY

NN5 representatives perceived the meaning behind interactions with Amber as being 'Christian-like' and appreciated how she worked within the complexity of the school environment.

Amber was seen to exemplify values such as kindness and respect. Community member three was a regular visitor to the school and regarded Amber as:

Living Christian values. For example, she concerns herself with fairness making sure everyone gets what they need to live and learn well. She practises Christian principles and the staff follow on from that. That's my perception. (ACA, C1SSI)

By noticing and responding to those in need, Amber helped build a culture where taking responsibility for those on the edges was the norm and further evidence of her concern for catering to diversity for belonging. Amber was also considered highly intelligent and a very good business woman. BOT Member Three thought that through penetrating questions and relevant remarks, Amber demonstrated a clear grasp of the complex nature of running a school.

\section{Nested Network 6-Aiga/whānau}

The task of the aiga/whānau NN was to support their children's education in a positive way and to help build a cohesive, sustainable school community. Amber was directly involved in supporting families to achieve this goal.

WHO

The membership of NN6 included:

- parents/older siblings/grandparents

- school neighbours

- parishioners.

Two parents and Amber provided data about NN6 and showed evidence of establishing connections with NN1, NN2, NN3, NN4, NN5 and NN7. 
WHAT

The main patterns of practice evident in NN6 included financial support and keeping communication open.

Firstly, finances were discussed. The MOE expect around $10 \%$ of school funds to be locally raised. Amber suggested in follow-up meeting five that perhaps $2 \%$ of what was needed was raised, meaning typically between $\$ 2,000$ and $\$ 8,000$. Rather than seeking money that was not available in the community, Amber reported in follow-up meeting six that she was constantly applying for grants to cover the costs of specific items, for example, computers, camps and school trips.

Statistics NZ (2013) showed that the average annual income for individuals living in the Acacia community was $\$ 17,900$ compared with the national average of $\$ 49,000$. Local aiga/whānau expressed gratitude for any financial and moral support received from Amber:

She understands people's circumstances, whether it's financial or whatever; she
tries to keep costs down in everything we do because she knows what it's like. She
makes activities, items, as cheap as possible. I need that help, sometimes just to get
my kid to school. (ACB, CSSI)

This quote is indicative of the idea that without financial support from the school, even having students present for learning was challenging. Free resources included shoes, daily breakfast, and the use of the school homework centre.

It was noted by some parents that financial assistance was flouted. Where parents could perhaps afford to pay for resources, some would not. Amber agreed that while this could happen, it was not the norm and supports were not withdrawn. Parent Four reported:

I grew up and my family was all on a benefit, but if I went to a school like this and all these activities and opportunities advertised were open to me... Maybe I might have done something different. I might have continued going on through school, but I didn't do that, and that's what I'm trying to make up for now. (ACB, CSSI)

This view represented many parents who were not only focused on meeting basic needs but also on utilising extra opportunities for their children, making up for their own limited opportunities. Amber concurred:

School is a place to enhance and enrich experiences for students... to offer an opportunity... to open a door. (PA, ISSI) 
Amber saw the role of the school as offering possibilities that may result in unexpected opportunities. This supports notions of educational success being about the development of the whole person.

Secondly, ways to keep communication effective ensured a connected network. Newsletters were issued fortnightly to the eldest child of the family and aimed to keep parents in touch with school and community activities. Facebook and Twitter were mentioned in community interviews and a two-page school pamphlet referred to an open-door approach meaning the school:

Encourages open communication, feedback, and discussion about any matter of importance to members of its learning community. (APA, FU5)

This is consistent with the emphasis Amber places on relationships. Amber reported in both follow-up meetings and during observation three that face-to-face conversation was one simple way to remove barriers so that everyone in the community could be heard. During the community meeting a parent said:

When my daughter was having problems, we would go and speak to the principal. She would go and speak to the principal too. A couple of times she rang up the school and said, I'd like to make an appointment to see Amber please. Students had been teasing her... Amber spoke to the two classes involved with her ... (ACE, CSSI)

Here, it seems that both students and parents felt they were empowered through their interactions with Amber and the actions she took. Aiga/whānau, and staff also noted an awareness of the amount of contact they had with Amber in a day:

She must get a lot of us in a day. Sometimes it can be to her detriment. We don't want her to get sick or anything and so sometimes she should start, you know, every now and then just saying "no I can't", like anybody must. She's got other stuff to do. (ACF, CSSI)

While the ongoing demands were recognised by some aiga/whānau as being unsustainable, and Amber reported that being accessible and offering a listening ear was tiring, she saw it as an essential part of her role. While these patterns of practice focused on developing goal two of the school charter, it became clear that the development of a home-school relationship was largely one-way.

How

Practice patterns were vulnerable when:

1. complex family needs were considered

2. the combination of insufficient MOE funding and the financial means of the community saw a large shortfall in school finances 
3. face-to-face meetings with aiga/whānau impacted on the principal's ability to complete other tasks.

The adjustments made in NN6 were as follows:

1. Amber was responsive to the support requested by families, ignoring what was perceived as 'taking advantage'

2. Amber applied for grants to cover the costs of specific things, for example computers, camps, and school trips, and spread the available finances further by not prioritising property needs

3. Amber accepted that face-to-face meetings were the preferred way used by aiga/whānau to explore any difficulties and improve student attendance. The sustainability of this was questioned by support staff and aiga/whānau.

Principal leadership in NN6 kept students at school, opened communication with families, and ensured that available funds were used wisely.

WHY

The NN6 representatives perceived the meaning behind network interactions as being about hearing the many voices of the community, giving discreet support and being fair-minded.

Firstly, Amber made time to listen to her community:

Amber encourages us to ask her anything, even suggest something. I'm never too scared to visit. She is never too busy and takes the time to talk and listen and we sort something out. (ACD, CSSI)

Amber is perceived as being approachable, giving parents the message that what they think matters. Additionally, Parents Two and Three describe Amber's approachability as like "coming home". This is consistent with previous statements that relate to Amber's willingness to establish strong relationships.

Secondly, support was also revealed by parents as fundamental:

We needed food and Amber was there to help us. It's not the first time she's done that. I have rung up before; we had to go to a funeral. Somebody had passed away unexpectedly and she was there right away. She'd come to my house and helped us out financially to get there. (ACB, CSSI)

Quotes such as these show, that without financial support, many families would be unable to attend to basic and cultural needs. Strong relationships built with 
aiga/whānau also meant that financial concerns were less likely to permeate students' lives and affect their studies.

Third, fairness was viewed as central to Amber's actions:

Everyone gets fair treatment. Everybody gets the same education. They've got activities like violin. She's got that there for everybody. (ACD, CSSI)

Many participants discussed Amber's efforts to be equitable so that opportunities and potential outcomes could be distributed fairly.

\section{Nested Network 7 - Education support workers}

The main purpose of NN7 is to support specific needs and interests apparent in the school on a short-term basis or, in the case of cluster colleagues, to provide ongoing support and share best evidence across the cluster in support of improved student and teacher outcomes.

\section{WHO}

The membership of this NN7 includes:

- music specialist

- Social Workers in Schools (SWIS)

- cluster colleagues

- literacy specialist

- art specialist

- engagement specialists

- school health nurse

- police youth liaison officer

- local religious groups.

Amber, the music specialist and SWIS provided data about NN7 and showed evidence of establishing connections with NN1, N2, NN3, NN4, NN5, NN6, NN8.

WHAT

The main patterns of practice in NN7 included resourcing and developing teaching and learning across the cluster.

Firstly, resources were constantly sourced. In follow-up meeting one, Amber spoke of the various opportunities that presented themselves: 
The local Buddhists make us sandwiches which get used for after-school care. Another example would be our PE equipment. It came from a high decile school who had lots. That means the PE budget for that year can be used for something else. Our specialist art teacher is also sourced from this school. She volunteers some hours here. (PA, FU2)

This quote shows that Amber's connections helped to address resourcing issues through redistribution of resources already held elsewhere in the community. It is the distribution of resources that supported previous statements about the desire to develop the whole child.

Additionally, practical support offered within this network may have ensured additional student opportunities were not lost. During a conversation with the police, Amber expressed an urgent need to transport students to a sports challenge as help with transport had not been forthcoming from parents. The officer agreed to use his resources to guarantee transportation. This suggests that NN7 addressed the school support needs where aiga/whānau could not and supports prior evidence pertaining to a gap in home resources.

Secondly, the network communicated about ways to develop teaching and learning across the wider community. The 'Reading Together Programme' was an education support programme which helped aiga/whānau to provide effective support for their children's reading. The programme was used at Acacia to develop aiga/whānau experience in reading with their children and was voluntary. It was noted in the researcher's diary, that Amber happily shared this information with colleagues in the wider community. Community member four reported how Amber grew the "Violins in Schools' programme, which started at Acacia and now involved seven local schools. These examples suggest that initiatives started by Amber led to broader network uptake.

Amber disclosed that an inter-school network, formed to develop teacher practice and expertise in writing, had disbanded after the three-year funding expired. The project aimed to address the writing needs of students across the cluster. While Acacia was no longer part of the writing moderation group formed during this contract, a new network emerged that focused on transitioning new entrants into school and Acacia remained a part of this initiative. 
How

Practice patterns were vulnerable when:

1. the uptake of additional opportunities was not supported by parents

2. programmes taken up at a wider community level struggled to sustain themselves.

The adjustments made in NN7 were as follows:

1. Amber capitalised on wider community relationships as they often created an alternative way of meeting or re-distributing resourcing needs

2. Amber continued to share programmes across the cluster, which instigated sustainability or emergence of the new in the broader network

3. principal leadership in NN7 reacted to tensions by maximising teaching and learning opportunities related to both the core curriculum (literacy and numeracy) and other areas such as the performing arts. Sharing the opportunities across the cluster showed the possibility of sustainability and emergence.

Principal leadership in NN7 created active boundaries where strong relationships could develop that would ensure greater sustainability of the network.

WHY

NN7 perceived their interactions with Amber contributed to a holistic, hardworking and socially-just approach to education at Acacia.

Firstly, Amber was concerned for the whole child and this is consistent with the views of some others in the network. Positive things were seen to be happening at the school including violin, band, and sporting opportunities. Spiritual wellbeing was also mentioned which offered what Education Support Worker Two felt was a palpable sense of care and respect.

Secondly, hard work was attributed to Amber who goes the extra mile but also trusted others to get things:

She inspires you to be the best you can be for better outcomes. (ACF, CSSI)

Here it appears that Amber worked on the assumption that hard work by herself and others would procure reward. 
Thirdly, an awareness of others' situations was noted by community member three who described Amber as socially just and viewed the entire staff as being compassionate. These ideas suggest an understanding of the civic responsibility required when leading in LSE contexts.

\subsubsection{Network Three: Wider Acacia community}

Network Three was made up of those who were rarely on site and typically supported Amber and the school indirectly. This network included, but was not exclusive to, private businesses. All data were derived from Observation One and the researcher or researcher's journal. These network members had no input into the meaning attached to Amber's work as no-one from the network took part in interviews.

\section{Nested Network 8 - Private businesses and organisations}

The purpose of NN8 was reported by Amber as providing contacts and resources for the management and leadership needs of the school. The NN supported Amber to widen the school learning community.

WHO

The membership of NN8 included:

- banks

- IT specialists

- trade services

- trusts.

Amber provided data about NN8. This NN showed evidence of establishing connections with NN1, NN3, NN4, NN5 and NN7.

WHAT

Providing funding opportunities to help future-proof school resources was the main pattern of practice evident in NN8. Communication with external charitable trusts was in evidence when a large donation was made to seven low decile schools in the area. The management of the $\$ 55,000$ was led by Amber who took the lead in futureproofing this gift and sought advice from an investment specialist.

Amber was observed applying for grants to help support the ICT infrastructure. One grant was made to purchase six new computers as well as to discard old ICT equipment at a cost of $\$ 25$ per monitor. Another grant application was underway to fund the replacement of the school server costing approximately $\$ 8,000$. This evidence 
suggests that resourcing basic future-focused requirements was the main purpose of the NN8 and required a lot of Amber's time.

How

Practice patterns were vulnerable when:

1. ongoing time was dedicated to finding alternative sources of funding

2. alternative solutions to future-focused ICT funding needs were slow and unreliable.

The adjustments made in NN8 were as follows:

1. The NN accessed private funds to offset the school's limited ability to fund big ticket items, such as the required ICT upgrades, but these were still unreliable

2. Amber managed various needs that were often in direct competition to each other by sourcing funding by any means possible.

Principal leadership in NN8 reacted to tension by being concerned for budget requirements, both now and in the future, without which regression would be likely. These practices were time consuming and relied heavily on Amber.

\subsection{Change trajectory}

Examples of the types of changes that occurred and how Amber's principal leadership network self-organised to maintain and progress itself when disturbed, became evident and can be seen in Table 11. 
Table 11: The feedback loops occurring in Amber's principal leadership network

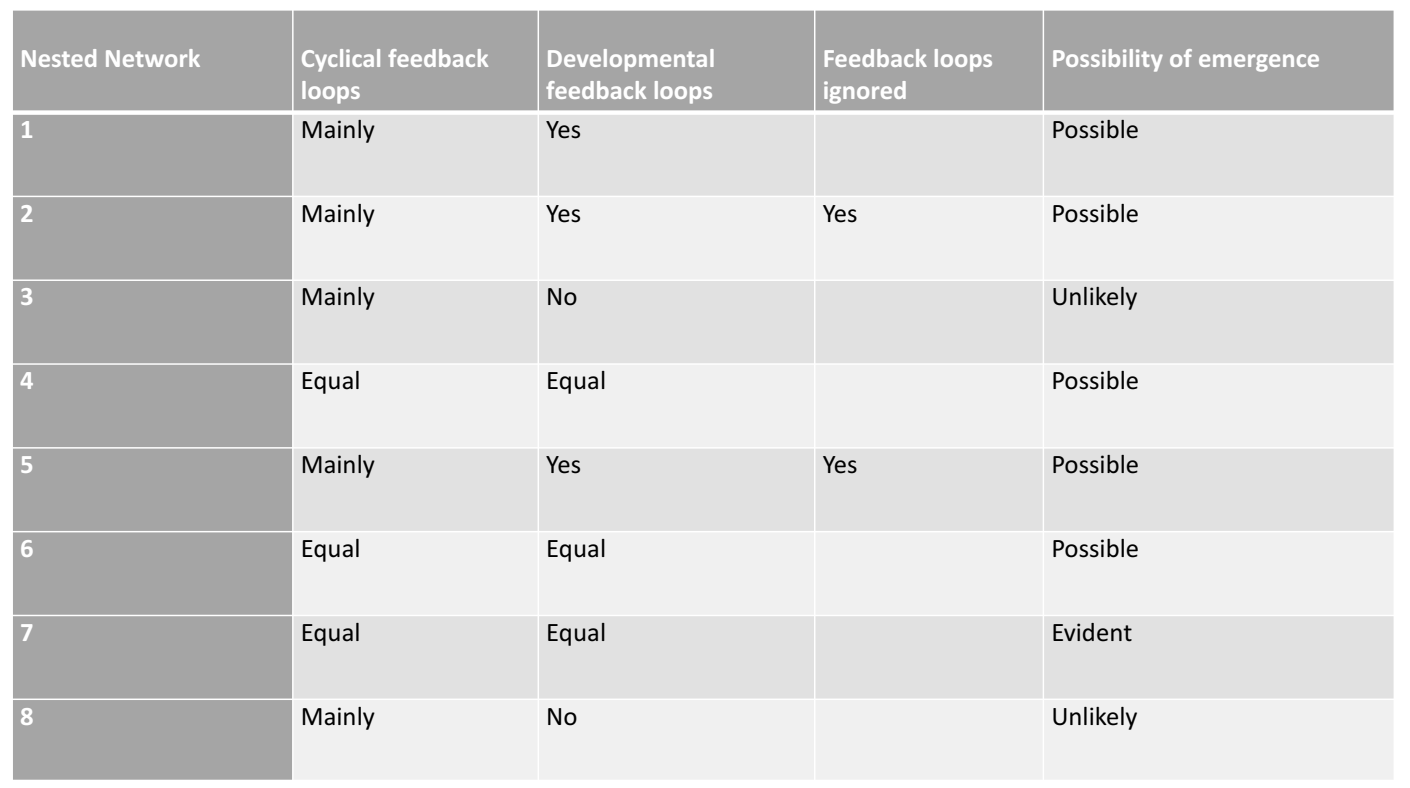

Cyclical feedback loops were seen to largely maintain status quo. These helped sustain the current patterns of practice and were included mainly in $\mathrm{NN} 1,2,3,5,8$. The feedback loops successfully worked to address issues with home-school relations, resourcing, PLD, student behaviour, safety, wellbeing and competition. Day-to-day, these areas appear most problematic.

There was also evidence of developmental feedback loops as a response to disturbances. This is where traction is gained - since responding to disturbances in this way would see new structures created. Developmental feedback loops related to homeschool relations and future-learning opportunities and were strongly evident in NN1 and NN2 and not at all evident in NN3 and NN8. The possibility of emergence appeared limited.

There was evidence of ignoring of feedback in NN2 and NN5. This occurred mainly with teachers in relation to parents' involvement and teacher and parent beliefs associated with student achievement possibilities. Deficit thinking is an ongoing concern at both a parent and teacher level.

There was one example of emergence in evidence in NN7 and this was the development of a transitioning programme. This emerged from a cross-cluster literacy project. However, in three of the eight $\mathrm{NN}$ at the least, emergence was possible, since there was evidence of developmental feedback loops in NN1, NN2 and NN5. Close 
collaboration between teachers, leadership and governance teams may be needed to achieve the likelihood of transformation of the school system.

Overall Amber's principal leadership trajectory is not regressing, mainly progressing and development with the possibility of transformation in support of more extensive educational reforms unlikely.

\subsection{Chapter summary}

Principal leadership practice at Acacia can be viewed as both a collective and individual construct and the sum of its parts.

\subsubsection{The principal leadership network}

The collective construct of principal leadership is bounded by shared relationships, understandings of educational success, and collective practices.

Educational success was perceived by the Acacia principal leadership network as being about developing the whole person both now and in the future. Being successful now saw students engaging in opportunities to develop personal strengths and making wide-ranging developmental shifts by setting goals and measuring movement towards these goals, while celebrating a feeling of success. Future success would see individuals who displayed a desire to keep on learning, fulfil dreams, and are socially responsible.

The principal leadership practice patterns were centred day to day around: engagement, the delivery of a holistic curriculum, PLD for staff inclusive of the principal, community wellbeing and learning, formative and evaluative assessment, budgeting, and school image.

\subsubsection{Principal Amber}

Principal Amber had developed a desire to 'do the right thing'. While she maintained a strong sense of where she had come from, her spiritual perspective was the foundation on which she based her leadership. Amber saw herself as someone who built strong relationships, had strong beliefs around diversity and belonging, and was a deep thinker who was solutions focused. These attributes aligned with the 
perceptions of others in the network. Especially cohesive across the network was Amber's ability to enact the belonging and inclusion of others.

As the network self-regulated, Amber showed strong evidence of collaborating, challenging and attuning her ideas and actions. There was evidence of Amber collaborating mainly with staff and support staff. She also offered encouragement to the governance body on matters of property and finance. Further, she released teachers, secured the school grounds, and shared resources, finances and programmes. Amber challenged her colleagues and aiga/whānau by pushing forward with initiatives and requesting other members to take on temporarily/different responsibilities. Amber questioned the narrative around deficit theorising and the engagement of aiga/whānau in school life. Additionally, humour was used to question or flag issues. Amber spent a lot of time addressing dilemmas across practice with an eye on the functioning of the collective whole. Amber used her strong relationships to address imbalances. More specifically, she responded to diverse family requests by improving welfare and school accessibility and ignoring negative teacher perceptions. Amber managed the school budget by managing the constraints or finding alternative funding sources. To redress a public perception, Amber worked proactively with governance to enhance the knowledge and reputation of the school while also celebrating Acacia's spiritual point of difference.

\subsubsection{Network capability}

The movement of the principal leadership patterns of practice looked towards cyclical change with the possibility of development and little evidence of emergence. The strength needed to create momentum for change that would propel the network into transformational possibilities was in fact being spent simply restabilising the network. This was often only possible because of the strength of relationships Amber maintained across the entire network. Constant disturbances in the environment meant that any good work was often being undone. When the burdens of a lack of aiga/whānau engagement, insufficient and reducing funds, inadequate expertise and increased accountability and competition between schools come together, the barriers to learning become insurmountable. Additionally, the progressive change identified might not be enough to withstand ongoing disruptions and could disrupt the network entirely. 


\title{
CHAPTER 6 \\ Principal leadership practice at Cedar School
}

\author{
"Breaking the cycle of poverty" Principal Cathy
}

\subsection{Chapter overview}

This second unit of analysis describes Cathy's principalship in interaction with the Cedar learning community. The chapter begins with a narrative of Cathy's upbringing, early career, leadership journey and personal philosophy. Next is a summary of Cathy's arrival at Cedar School and community notions of educational success. I then describe the nine NN that make up Principal Cathy's leadership. The connections that occur across these NN from within the localised LSE setting show the layers of added complexity. In the main body of the chapter the composition and patterns of communication detail who is in the network and what is discussed; any disturbances and adjustments are also described to show how the NN moves and re-organises itself. Where possible, perspectives related to meaning are included. The change trajectory is inferred. The chapter concludes by summarising Cathy's principal leadership as a collective and individual construct and the likely contribution it is making to wider education reform.

\subsection{Abbreviations}

The following abbreviations were assigned to the Cedar School research participants.

Table 12: Cedar School abbreviations of data sources assigned

\begin{tabular}{|c|c|c|c|c|c|}
\hline Code & $\begin{array}{l}\text { Data sources at } \\
\text { Acacia School }\end{array}$ & Code & $\begin{array}{l}\text { Data sources at } \\
\text { Acacia School }\end{array}$ & Code & $\begin{array}{l}\text { Data sources at } \\
\text { Acacia School }\end{array}$ \\
\hline PC & Principal Cathy & & & & \\
\hline CSG & $\begin{array}{l}\text { Cedar staff } \\
\text { group }\end{array}$ & CCG & Cedar community group & CD & $\begin{array}{l}\text { Cedar school } \\
\text { document }\end{array}$ \\
\hline CSA & Cedar staff $A$ & CCA & Cedar community member $\mathrm{A}$ & CD,SC & $\begin{array}{l}\text { Cedar school } \\
\text { document - school } \\
\text { charter }\end{array}$ \\
\hline CSB & Cedar staff B & ССВ & Cedar community member $\mathrm{B}$ & & \\
\hline CSC & Cedar staff C & $\operatorname{CCC}$ & $\begin{array}{l}\text { Cedar community member } \\
\text { C }\end{array}$ & CS & Cedar student group \\
\hline CSD & Cedar staff $D$ & CCD & Cedar community member $\mathrm{D}$ & & \\
\hline CSE & Cedar staff E & CCE & Cedar community member $\mathrm{E}$ & & \\
\hline CSF & Cedar staff F & CCF & Cedar community member F & & \\
\hline CSG & Cedar staff G & CCG & Cedar community member $\mathrm{G}$ & & \\
\hline CSH & Cedar staff $\mathrm{H}$ & $\mathrm{CCH}$ & Cedar community member $\mathrm{H}$ & & \\
\hline
\end{tabular}


The abbreviations in Table 12 above, can be found throughout this chapter alongside the data method codes developed in Section 4.8.4. Together these codes are evidence of the data used to confirm findings.

\subsection{Cathy}

Cathy discussed her upbringing, early career and educational leadership journey and what inspired her practice.

\subsubsection{Upbringing}

Cathy grew up in a working class, Catholic household with parents who were deaf. She left high school at 15 to be an apprentice cutter. However, Cathy was influenced by her brother and a colleague to return to tertiary education. She enrolled at Teachers' College, where she completed a three-year teaching diploma and several university papers.

Cathy's first year of bonded ${ }^{28}$ service was very successful. Her second year however, was more challenging, and while she stayed on to see out her bond, she left teaching and established a career in the public service.

After some years working for the Ministry of Women's Affairs, Cathy travelled overseas where she returned to teaching:

I enjoyed teaching overseas and I felt like I was a successful teacher abroad. I even did some university study and came away with a Diploma in Urban Education. (PC, ISSI)

Once back in New Zealand, and following some time as a reliever, Cathy won a permanent position at a low decile school. A seed was planted by a colleague regarding deficit thinking ${ }^{29}$ and the difficulties that come with it. Cathy concluded:

Racism is a white problem. (PC, ISSI)

This comment shows the awareness Cathy developed in relation to ethnicity, citing discrimination as strongly positioned within the status quo.

\footnotetext{
28 While teacher training was free in New Zealand, teachers were bonded upon graduation, meaning they taught in government schools wherever required for two years.

29 Deficit thinking is a term that explains students failing because of their perceived deficiencies.
} 
Cathy left to have her first child and, while on maternity leave, attended a full immersion 12-week Te Reo Māori ${ }^{30}$ course. After having her children, she returned to teaching full time.

\subsubsection{Leadership journey}

Cathy's formal educational leadership experience began when she went on to become part of a liaison resource team. ${ }^{31}$ From this brief reprieve from teaching, Cathy became a Deputy Principal, noting that the opportunity arose because of a change from the 'time-served' pre-requisite for principal appointments to merit-based appointments. Cathy later began applying for principal positions. Although previously uninterested, she came to realise she did want to take on such a position and settled into eight years of service in a mid-decile area. The local school community, however, was fractured and the school identity under threat:

I could see that many of the parents who lived outside of the area wanted an alternative education, and saw the potential for having their own school. However, the local parents were keen to keep their school as a local school delivering the New Zealand Curriculum and meeting the unique needs of Māori whānau. I thought, I'm not going to let this school become anything other than a local state school, but that surely, we can shape it to meet the diverse needs of all students who attended. With Tomorrow's Schools, there's a risk of middle class capture, of not recognising the needs and aspirations of all students and their families. (PC, ISSI)

Here, Cathy showed her awareness of the gap between the intent of policy and what occurs in practice. She worked hard to improve responsiveness to, and accountability for, all those in the community - especially those at the margins. She persisted with the idea that a local school must stay local:

A beautiful and very precious environment emerged. I didn't realise how much I grew the school until I left and saw it from the outside. (PC, ISSI)

This quote suggests that her innovative stance was only able to be identified in hindsight. Cathy also learnt about how to work with others:

Collaboration is much deeper than working alongside someone. It has taught me to listen and make sure not to undermine others' work. (PC, ISSI)

She then worked for ERO and reported that she observed many high-performing schools in her time there. She also upgraded her formal qualifications to a PostGraduate Diploma in Evaluation.

30 Māori language.

31 In the 1990s liaison resource teams were stationed within local communities and worked across schools to offer extra PLD opportunities and address any resourcing needs. 


\subsubsection{Cathy's philosophy}

The semi-structured interview and follow-up meetings provided a window into Cathy's personal and educational philosophy and included several key beliefs: awareness of the big-picture; connectedness; and a learner disposition.

\section{The big picture}

Cathy spoke of the importance of retaining the big picture so important alternative viewpoints might be heard.

In follow-up meetings two and three, Cathy expressed her cognisance of the wider society influencing the network/s within which she worked. With personal experience of how prejudice can affect one's ability to achieve, she discussed the negative influences of deficit theorising, for those of ESL or minority ethnic origins and for females. These are all ideas that Cathy said promoted competition and individualism:

Policies in New Zealand make it impossible to have needs met, even when people have all the cultural capital in the world to do it. That's inequity. (PC, FU1)

Cathy's understanding of a bigger picture and what she referred to as a neoliberal approach meant she recognised those in her community were given less choice to utilise their skills.

Challenging one's perspective and adjusting to different ideas were examined by Cathy through her role as principal. This sometimes meant questioning the status quo but also showing a willingness to try to see the world through the eyes of others:

I'd hear, 'the main problem is they don't speak English'. I made myself unpopular by repeating this phrase, 'No, the main problem is that you don't speak Samoan'. (CPC, ISSI)

The quote suggests Cathy shifted her perspective from a colonial construct to a more inclusive ideal, verbalising and using it to challenge others. This is consistent with the previous quote referring to cultural capital and inequity.

\section{Connectedness}

Cathy valued cohesion and connectedness. A cohesive framework does not just occur. Instead, Cathy stated:

Finding all those links. We have got to weave ourselves together. (PC, FU2) 
Cathy's expectation was that teachers, students and their families were willingly part of the school learning community and sought to develop a shared culture, demonstrating 'how things are done around here'.

\section{Learner disposition}

There was a strong expectation that adults are learners too. Cathy spoke of a strong pedagogy and getting teachers thinking and acting in a considered way, with a clear purpose of drawing on research for improvement of students, and creating deeper learning opportunities:

\section{I want them to say, 'I am not doing this because you told me to but because it has a} purpose that makes sense to me, and it's better for the students'. (PC, FU2)

Continually seeking challenge and questioning what is going on meant teachers were invested in the complexity of learning and improvement. When speaking of a teacher's desire to improve, Cathy said:

If you are not looking for the challenge you are not doing a good job. (PC, ISSI)

This quote underlines Cathy's view that asking questions constantly was evidence of a positive learner disposition.

\subsection{Cathy as principal of Cedar School}

After completing five years with ERO, Cathy arrived at Cedar School in 2008, an school in which she had previously relieved. She started as the Deputy Principal and later became principal. Cathy attested to having:

Nice relationships with everyone. I aim to create an environment of love, belonging, and freedom. (PC, ISSI)

This comment exemplifies the influence of Glasser's (1999) choice theory ${ }^{32}$ and Cathy's focus on three of the five basic needs.

Cedar School is a full primary school. Buildings are maintained to standard and plans are in place to modernise classroom spaces and resources. The school vision was evidence of the entire community's ideas about wanting to develop students who were:

Confident, connected, actively involved, environmentally aware life-long learners.

$(\mathrm{CD}, \mathrm{SC})$

32 Glasser's choice theory considers human behaviour as purposefully based on internal motivators of survival, love and belonging, power, freedom and fun. 
The charter was streamlined into four goals and these were:

1. Raising student achievement

2. Achieving high quality teaching

3. Developing relationships

4. Developing mutually beneficial partnerships between the school and its community

The vision and school goals showed high expectations for learning both now and in the future. A strong identity and being successful academically were the two themes evident in the charter.

To achieve the vision and goals, Cedar School focused its school three-year plan/s on a curriculum built on principles of: respect, innovation, equity, ecology and integrity, and values including aroha, ${ }^{33}$ awhina, ${ }^{34}$ and manaakitanga. ${ }^{35}$ These principles and values acted as the framework for teaching and learning. Key competency skills included: self-management, relating to others, and an ability to use digital technologies. The key areas of learning from the National Curriculum Framework were used to contextualise the teaching of the above principles and values, and included literacy, numeracy, science, technology, the arts, health and physical education.

When asked to use a metaphor to describe the leadership used to reach such goals, Cathy likened it to that of a weaver (Figure 7) and explained how she hoped to help students and families experience belonging and success so they could break the cycle of poverty.

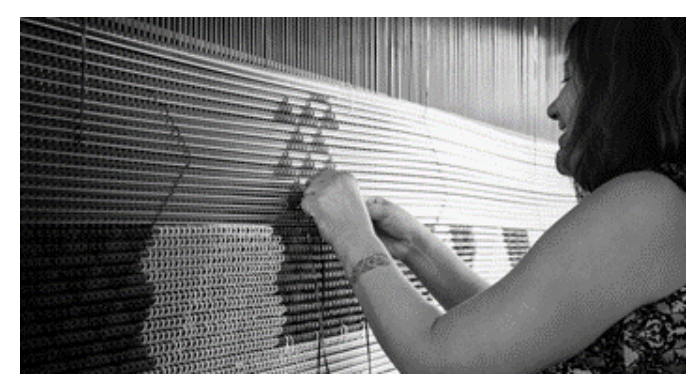

Figure 7: How Cathy described her work as principal leader at Cedar School

33 Māori for love

4 Māori for support

35 Māori for hospitality/kindness/care 
Cathy attempted to weave the learning community together for greater presence, engagement, and success. She stated:

I want them to be happy people and build the lives they want. My role is to be aware of the neoliberalist approach which has exacerbated unfairness and help families access the resources needed to change it. I can help them access the supports to make sure they can do their jobs as parents. (PC, FU2)

This quote shows that Cathy saw her role as a leader who worked towards greater possibilities of educational success for students in poverty, who she believes are being punished. Cathy saw education as a key factor in preventing poverty:

Whilst it's not my role to get families out of poverty, it is my role to help them access the resources needed so they can get themselves out. We are part of the solution for getting children and their families out of poverty because a good education can help with that. (PC, ISSI). By making resources and supports available, Cathy believed she could help break intergenerational poverty cycles.

\subsection{Educational success in the Cedar community}

The Cedar community was asked for their ideas on education success. Drawn together were perspectives from the staff including Cathy, students, the community, and parents. School documentation was also variously called upon. Characteristics of educational success included academic improvement, key competencies ${ }^{36}$ and development of the whole person.

\subsubsection{Academic improvement}

Education success at Cedar was acknowledged, formally, as reaching standards set by the MOE or the School. Cathy states:

Formal qualifications may only be one part of success but for many students it's a ticket to a range of future options. (PC, FU5)

Parents, trusts and private industry representatives concurred and saw success in the basic areas of reading, writing and mathematics as essential skills for success later in life.

Less formally, achievement meant surpassing a prior personal attainment level. Staff used the word 'improvement', or 'reflection' and expressed a desire to see students

36 Key competencies are interdependent knowledge, skills, attitudes, and values and help teachers to understand how students are learning. 
moving forward. The process appeared as more important than the outcome or the empirical results.

Students emphasised the development of their ICT knowledge as an indication of academic achievement, because they said it helped them to 'learn faster'. Academic success included attainment of essential skills in reading, writing, maths and ICT. This type of success required full engagement in the learning process, creating forward movement.

\subsubsection{Key competencies}

Key competencies showed the behaviours a student might demonstrate as being educationally successful and according to teachers and parents included: participating and contributing, self-managing, and relating to others. Cathy summed this up as:

Showing a curiosity about the world. (PC, FU6)

Learning behaviours that give evidence of engagement were viewed as an important part of being a successful learner.

The development of thinking, considered the most vital of the key competencies by participants, would allow the learner to have an awareness and understanding of personal learning needs and:

About one's own thought processes. (PC, ISSI)

Cathy emphasised this as a way of developing confidence to help students when they could not do something. A teacher noted:

They like feedback. That's what they want the most, not just - good girl! They want the feedback. They want to improve, to set their own direction and use their initiative. (CSA, SSSI)

This comment suggests that it is the actions of the teacher and perhaps the quality of their feedback that ensured full participation in the learning process.

Teachers further spoke of their influence over the quality of the learning, referring to the need for students to fully understand the purpose of their work: 
Here, staff were suggesting that by supporting students to have a clear purpose, independent student action towards a self-nominated outcome was more likely. Whānau and Trust Representative One added that by students showing greater engagement and self-management now, the likelihood was that they would have successful secondary school careers and even successful adult lives.

The key competency 'relating to others' was most talked about by students and aiga/whānau. Students identified:

Belonging, feeling safe at school, making new friends and having fun learning. (CS, $\mathrm{SA})$

It appears that by interacting positively with others, students were more likely to feel safe and be ready to learn. Community member $\mathrm{C}$ added that positively relating to others might mean students were more likely to stick to the goals they set themselves. Social support may therefore encourage higher expectations.

Key competencies focused on student engagement behaviours and included participating and contributing ideas, self-managing, and relating to others. This type of success requires teacher feedback and a clearly communicated purpose. With improved key competencies comes improved metacognition and ongoing intrinsic motivation.

\subsubsection{Development of the whole}

There was a strong focus by Cedar's networks on the whole child:

Students being offered an opportunity to shine in whatever ways suit them. (CSB, SSSI)

This quote is indicative of the value attributed to an education that combines students' mental, physical, social, emotional and spiritual stimulation. Cultural identity was singled out as adding value rather than a barrier to potential success:

Māori achieving as Māori, Samoan children achieving as Samoan and trying to as best you can, as an outsider - see the potential for that child. (CSC, SSSI)

The school enrolment pack showed success as inclusive of competency in a second language. An emphasis on understanding Toi Te Tiriti $O$ Waitangi ${ }^{37}$ was also noted.

${ }^{37}$ Māori for Treaty of Waitangi. 
The principal, staff and aiga/whānau talked about an intangible factor, that of success as a feeling. Cathy believed that if students felt successful, they would want to explore new things, while students themselves simply noted feeling 'proud' of their efforts. Staff talked about teaching students to listen out for the feeling of success. Parents additionally touched on happiness. All these were considered indicators of, and contributors to progress.

The development of the whole included a variety of facets of a person. This type of success at Cedar is inclusive of cultural identity and an intangible feeling of success unique to the individual.

\subsection{Representing Cathy's principal leadership network}

In this section, I represent both the outer and inner form of the network. The outer network is made up of three smaller networks and those three smaller networks are made up of nine NN. The inner form of the network shows how NN connect and move across the various boundaries.

\subsubsection{The outer network}

The outer network shows three smaller networks that became evident during a twelvehour day, a day Cathy described as 'untypically quiet'.

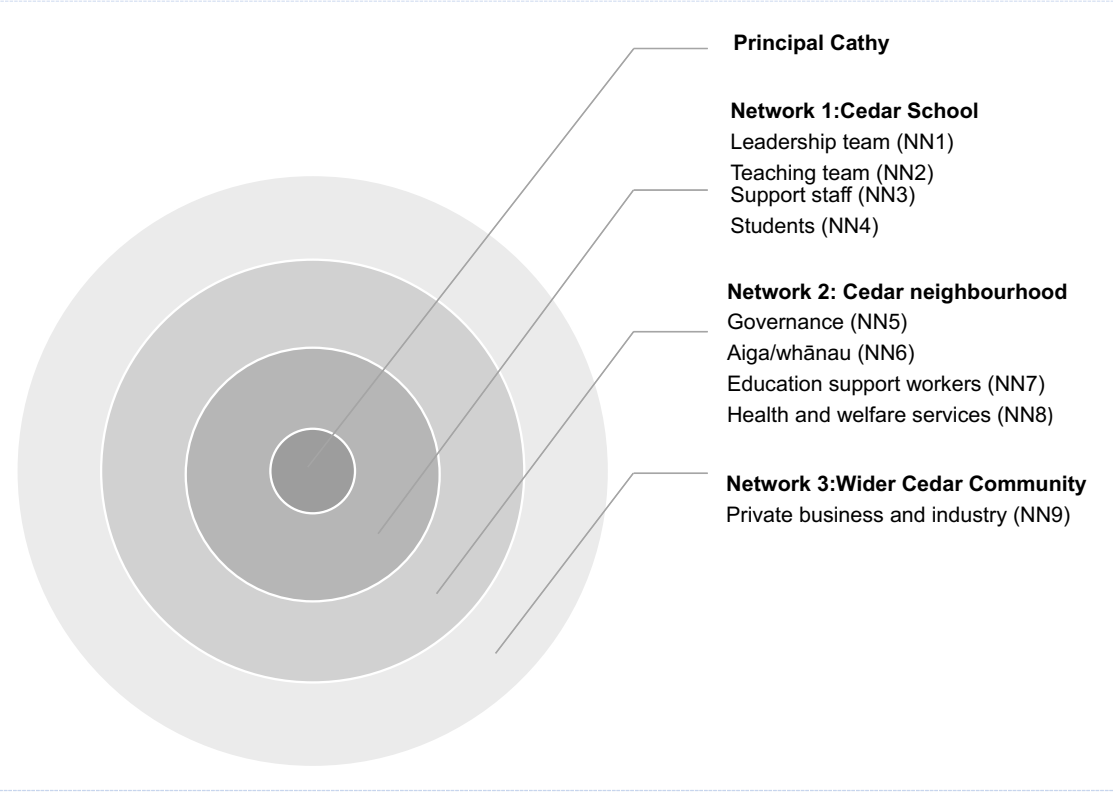

Figure 8: The networks and NN evident in Cathy's social relationships across a day 
- Principal Cathy has a system of her own which includes aspects of her upbringing, career and leadership journey previously described

- Network One included: the leadership team, teaching staff, support staff and students.

- Network Two was inclusive of BOT, aiga/whānau, and health and social workers.

- Network Three was comprised of trusts and private industry. It had a wide variety of aims inclusive of providing mentoring and support for Cathy, helping to sustain a healthy teaching and learning environment, and developing resources.

Figure 8 gives an indication of the groups of people Cathy draws into each of the three wider networks of her principal leadership practice. Cathy had self-directed boundaries. That is, Cathy determined who she interacted with, the number of relationships and connections she maintained, and who is, and is not, included in the network.

\subsubsection{The inner network}

The inner network describes the connections that took place across the NN in a day. Each NN was represented in the research by a variety of groups, with each group having their own code. Cathy was part of each NN, so this representation is uniquely hers.

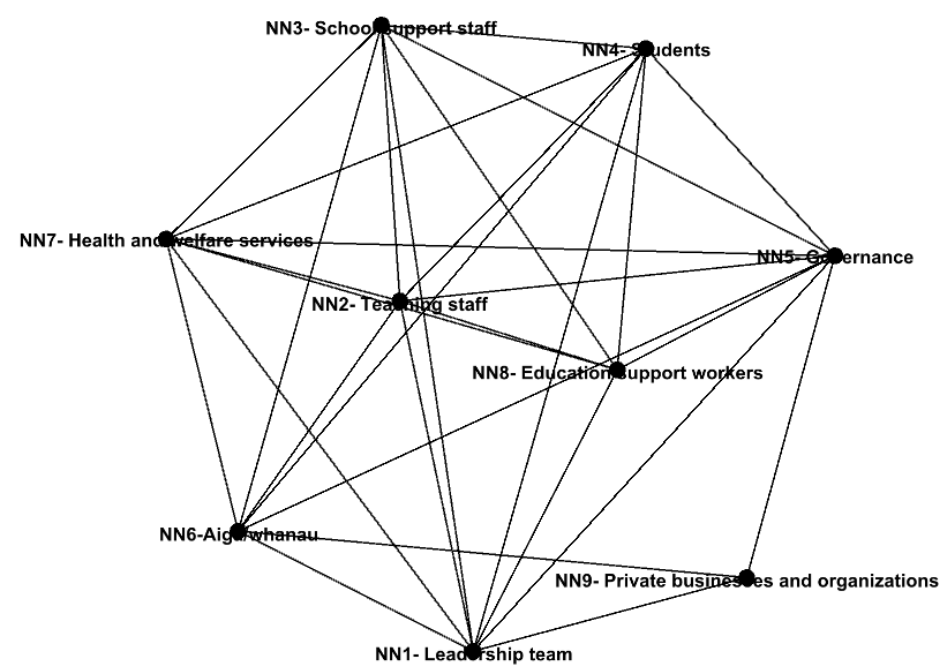

Figure 9: The NN connections from within Cathy's principal leadership network 
The following points are made regarding the forming of principal leadership practice as a group activity seen in Figure 9:

- the network is highly interconnected. Aiga/whānau had a total of 28, school support workers and students 26, and leadership team 25 interconnections. Additionally, governance had 24, teaching staff 22, and health and welfare 21.

- the network was made up of multiple connections between NN. While private businesses/organisations had only 3 of a possible 9 connections, all others having between 7 and 9 .

- connections are considerably further apart between: school support staff leadership; health and welfare - governance; students - aiga/whānau /leadership team.

- the closeness of connections shows a strong sense of familiarity between teaching staff, educational support workers, and health and welfare services.

Aspects of degree centrality, closeness centrality and betweenness centrality (explained in the methodology chapter) all show that the nine NN are connected, interconnected and work together for a stable principal leadership network.

\subsection{Describing the network}

The quality of the network is now described. Drawing mainly on observations and interviews, but at times documents, representative examples of how each NN responded to any contextual disturbances and then self-corrected to keep the purpose of the network in focus, are described. Where possible, how the informal structures are perceived is explained by those belonging to the network/s.

\subsubsection{Network One: Cedar School}

Network One is the Cedar School Community and included representatives from the leadership team, teaching team, support staff and students. The NN within are labelled $1-4$. 
Nested Network 1 - Leadership team

The purpose of the leadership team was to help guide school-wide decisions in support of improved student achievement.

WHO

The team of six had been together since Cathy started her principalship in 2009 and included:

- Senior Teacher One - led the senior school whānau

- Senior Teacher Two - led mathematics and the junior school whānau

- Senior Teacher Three - led literacy and oversaw the school library

- Senior Teacher Four - led ICT and the middle school whānau

- an Administration Manager - provided administrative support

- Cathy - recognised strengths in leaders and provided oversight of school culture, organisation, and curriculum.

All NN1 members provided data and showed evidence of establishing connections with all other NN.

WHAT

The main patterns of practice evident in NN1 included extending the curriculum, developing teacher learning opportunities, and adapting school organisation.

Firstly, the senior team communicated about the planned curriculum. During Observation Two key areas of learning were discussed in detail and included mathematics, science and digital technologies. The importance of protecting school time dedicated to these learning areas became apparent during a senior team discussion regarding a health initiative that offered free probiotics as part of a nationwide study on sore throats. The time taken to administer the probiotic was identified by the administration manager as having the potential to disrupt planned literacy, numeracy and languages. The team put a plan in place to ensure the opportunity to be involved would not be missed but would also not encroach on class instructional periods.

Secondly, the team communicated about PLD. It was noted that it was difficult to access appropriate resources to address a cohesive PLD programme that would cater for both individual and collective needs, especially in ICT and science. While resources offered by the MOE included funding, the content knowledge imparted did not necessarily address staff knowledge gaps or contextual considerations. Cathy 
reported the senior team was clear about what was needed and suggested that they develop their own internal plan and divert funds coming into the school for other purposes. One member of the team suggested contacting crown ministers directly to find a way to access up-to-date ICT content knowledge and support, since this was necessary to move forward with staff ICT PLD needs. In this instance, the team problem-solved together, with Cathy seen to be encouraging of the use of internal adaptations and political lobbying which would achieve timelier and more appropriate PLD.

Discussion about external teaching and learning opportunities that supported the broader school vision also occurred. Opportunities were spoken of at length in Observation Two and included mentoring a Masters student for a year and being involved in national mathematics research. Cathy also expressed an interest in some Chromebook research with the local university, which she believed had the potential to help measure the impact of the Chromebook initiative already in place. In followup meeting three Cathy commented that whilst coordinating efforts with outsiders was time-consuming, such involvement had multiple benefits, including the chance to evaluate initiatives already in place and bring extra resources into the school. Cathy thought that being involved in the MOE maths research meant that the school might:

Eventually stand as a centre of excellence in the field of maths. (PC, ISSI)

This shows PLD that would likely go beyond leading the local community into a leadership role at a regional and national level. External opportunities offered the potential of influence outside of the immediate school context.

Thirdly, school organisation was discussed. Near the end of each year, the leadership team looked at projected student numbers and staffing allocations before drafting class lists. These were distributed amongst the leadership team. The aim was to arrange teacher attributes and skills that might best suit student needs. Conversation continued into the New Year and it was decided a structural solution would be instigated since projected staff numbers, changes to teaching philosophy, cultural competencies, content and skill levels of teachers, and the increasing expectation for students to learn and demonstrate high levels of self-management and relating to others made school reorganisation a much more pressing issue. A change from four whānau groups to two was initiated to address many of the considerations listed above. 
How

Practice patterns were vulnerable when:

1. serendipitous health and wellbeing opportunities were utilised

2. PLD opportunities and resources did not meet the needs of all teachers

3. consensus could not be reached about grouping to address both student and teacher needs.

The adjustments made in NN1 were as follows:

1. The $\mathrm{NN}$ ensured minimal interruption to the classroom timetable

2. The leadership team developed an in-house PLD plan to ensure the development of teacher quality. Flexible and multi-functional relationships between staff, the senior team, PLD providers and other education institutions were developed. Cathy additionally encouraged the lobbying of politicians

3. A new school-wide structure emerged which suited various needs.

Principal leadership in NN1 made it possible to balance planned curriculum with efficient use of teaching expertise and to take opportunities that addressed current and future PLD resourcing needs and to redevelop school organisation.

\section{Nested Network 2 - Teaching staff}

The purpose of NN2 was to increase student wellbeing and academic success. Teaching staff included those who had direct contact with students and their learning. WHO

Many members of NN2 had worked at Cedar for some time and included:

- $\quad$ seven classroom teachers, three of whom were also senior team members

- a library teacher

- library assistant

- up to six teacher-aides. Three of these taught the community languages of Samoan, Tokelauan and Cook Island Māori while also working with individual students. One teacher aide taught extension Te Reo Māori for Māori students - Cathy, who taught where necessary.

Principal Cathy, all classroom teachers and one TA provided data about NN2 and showed evidence of establishing connections with all other NN. 
WHAT

The main patterns of practice evident in NN2 included sharing a vision, professional development, formal assessment requirements, and preserving connectedness.

Firstly, staff spoke at length about a shared vision and feeling very connected to each other:

We have a very clear school vision. This came out of some of the stuff that we've done recently. I thought it was cool, too, that we all came up with the same things. (CSE, SSSI)

A tension, however, existed between the school vision and how the public viewed Cedar's efforts. This was commented on by Teachers Two and Five, and was later expressed by Cathy:

We are all useless here. Useless teachers, useless kids, useless parents. ...We are always the reason our kids are failing... I hear questions like - why would rich parents send their kids to your school? Because it's a good school! (PC, FU4)

Many teachers agreed that there appears to be a mismatch between how the wider public view the quality of the teaching and learning at Cedar, and the internal views on teaching and learning at Cedar.

Cathy spoke of continuing to develop the value of the Cedar community by seeking input from the local mayor to fund a pamphlet drop promoting Cedar School and one other school close by, as great schools. The previously mentioned nationwide PLD involvement would possibly also emerge into a more positive view of learning at Cedar.

Secondly, staff commented that teacher development at Cedar was encouraging of enquiry into practice to see what worked. There was a shared belief that their work was complex. During Observation One, Cathy was seen visiting classes to keep abreast of what teachers were trying out. From Cathy's involvement in observations she noted a need to restructure planning sheets so that key competencies were the focus rather than curriculum areas. Cathy showed a strong trust in good outcomes and, as noted by various staff members:

Gives us a freedom - a freedom to try new things, to be creative. We are never bored and always learning lots and lots. (CCD, SSSI)

This quote is typical of how staff see the link between solving complex learning dilemmas and creativity. A tension, however, appeared to exist between making 
improvements and the speed of deployment. The creativity needed to understand complex learning needs meant much time was spent exploring different ways of doing things, yet there was an accepted need for teaching staff to do things better and with urgency:

I'm on a waka that I really want to be on. Sometimes I think the waka is going too
far, too fast and that's probably something that I struggle with a little bit... you are
paddling really fast to keep up whether you've got the right paddle or not... or using
your hands. It even feels sometimes like there's a hole in the waka and the bailer is
not there. But we get tighter as a group, look after each other.... to start with, Cathy
decides on the direction and then supports when the rest of us manage to get our
feet into the waka and paddle. But she continues to support.... it is a big boat. It's
going to be a cruise liner soon....as long as it doesn't sink or run aground or
something....it does run into the shallows every now and then, we've managed to
escape being, you know, totally capsized. (CSE, SSSI)

This quote, illustrates Cathy's beliefs about a strong leader pushing boundaries regardless of the disequilibrium it creates. Continuous and rapid change is necessary to solve the issue of improving outcomes for students in such a complex scenario, but it also does result in feelings of insecurity for teachers. While Cathy was concerned for the staff's health and wellbeing, she mentioned in follow-up meeting two she was unwilling to slow down to make things comfortable for teachers - getting it right could no longer wait.

Thirdly, formal assessments were discussed. Teachers monitored student progress using everyday observations and discussions but also through more formal assessments. During Observation One a staff meeting took place, in which Cathy was seen developing her mathematics assessment knowledge alongside others. The moderation meeting helped staff develop a shared understanding of numeracy achievement across the school by listening, asking questions and entering debates regarding improvements, gaps, and target groups.

During interviews, however, teachers themselves expressed reservations about the levelled assessments used, this time in writing. Staff felt that the variations they had observed in how tests were administered and marked across the cluster, were suggestive of incomparable and unreliable results. It was reported by staff member two, that Cathy supported them to change their formal assessment decisions to match their new learning. Staff Member Four expressed some confusion over the suggested changes, wondering whether this was leading to being dishonest with their cluster colleagues about the contribution this made to students' overall NS results. This 
evidence suggests that using one tool to measure student achievement and develop teacher learning was creating inconsistencies and teacher uncertainty.

Fourthly, expectations of connectedness were communicated. Staff reported feeling especially connected to others as a means of support and guidance:

These kids are our kids. Cathy values whānau connections. It's mostly the teachers that are doing it but she's pushing to make those connections, she'll support us to make those connections, like with our maths evening. It's something that she believes really, strongly in and you know she'll get us to make those links. Give aiga/whānau a call. You know, keep in touch. (CSB, SSSI)

This quote indicates that Cathy expected teachers to help create the same feelings of support and belonging for aiga/whānau in the community, adding further evidence to her strongly held belief of connectedness.

A possible disturbance to the intactness of the network was the dropping school roll. While provisional staffing rounds provided a minimum level of entitlement for the upcoming year, Cathy predicted a falling roll which would result in a loss of staff and reduce her salary. ${ }^{38}$ Cathy employed an outside facilitator to facilitate a disestablishment process of teaching positions. With the BOT unable to decide about which staff member should be made redundant, Cathy initiated several budgetary and job description changes so that all teaching staff could, in fact, remain. The disestablishment process was thought by Cathy to have:

Put some pressure on the school's finances and affected classroom teachers, since some teachers saw the process as unnecessary. (PC, FU3)

This quote indicates that financial stress put the culture of care in danger. Cathy held meetings with teachers who requested them, to give reassurance and answer any ongoing concerns.

How

Practice patterns were vulnerable when:

1. there was a mismatch of expectations between the internal view of whether the school could effectively meet goals set and the external views held

38 Principals' salaries in NZ are regulated according to school grading. The grading is largely determined by roll. 
2. the speed at which solutions to more complex problems could be found

3. inaccurate assessment data created a lack of confidence in assessment results

4. there was a decline in the school roll.

The adjustments made in NN2 were as follows:

1. Cathy connected with city councillors and researchers to influence outsider views on the Cedar area, and the quality of education at Cedar school

2. Cathy acknowledged but did not alter the speed at which changes were being deployed.

3. the moderation meeting helped staff develop a shared understanding of numeracy expectations

4. Cathy noticed the concerns teachers had regarding the disestablishment process and responded by involving staff in the process.

Principal leadership in NN2 balanced welfare and learning needs as an and/both requirement, pushed for change, prioritised teacher learning, and developed a strategic approach to balancing the school budget so school programmes, teacher morale and wellbeing could continue to develop without financial hindrance.

WHY

$\mathrm{NN} 1$ and NN2 representatives perceived the meaning behind the network interactions as being about developing knowledge, a challenge to teaching practice, and an inclusive school environment.

Firstly, staff spoke of Cathy’s knowledge:

She knows so much about so much, she pulls stuff from everywhere ...various theories such as Glasser's choice theory ... but also remembers what good practice looks like. She encourages us to read and understand and be involved. (CSE, SSSI)

These comments suggest that Cathy used research to inform and illustrate her views but worked to link this to teachers' first-hand experiences. Together, research, practice and context were considered when putting theory into practice.

Secondly, staff focused on challenges to teaching and learning: 
ideas and so that's where you end up, well (you know) ... that it's been well thought through. (CSB, SSSI)

Here challenge, as modelled by Cathy, and practised by others, is viewed positively as it leads to greater depth of thinking.

Thirdly, staff spoke about an inclusive school built on teamwork:

The waka doesn't go anywhere without paddles and paddlers. But she is on it too. She works as hard as we do. (CSE, SSSI)

Teachers agreed that everyone is as important as each other and that Cathy was very much a part of this group. As a result, NN2 made sense of principal leadership as being about developing a depth of knowledge, challenge, and support that kept the network focused on learning.

\section{Nested Network 3 - School support staff}

The purpose of NN3 was to ensure an orderly school environment and support liaison between home and school.

WHO

The membership of NN3 included:

- the office manager

- the caretaker

- canteen manager.

The office manager provided data about NN3 and showed evidence of establishing connections with NN1, NN2, NN4, NN5, NN6, NN7 and NN8.

\section{WHAT}

The main pattern of practice evident in NN3 was student attendance.

The office manager was observed during Observation One keeping a close watch on student attendance and the school community goal shows the main reason for this:

To increase the average level of attendance for each child in the school to between 95 and $100 \%$ and to also reduce the number of students with an average attendance level of $85 \%$ to 0 . $(\mathrm{CD}, \mathrm{CC})$

An absenteeism rate of approximately 50\% above the national average, in the wider Cedar area, was the main reason for the goal. Attendances and absences were logged electronically. Cathy agreed to follow up with families and subsequently, the school administration manager, on any unexplained absences. She said: 
Students may be absent due to a lack of food or clothing, bad weather, looking after younger brothers and sisters, overly protective family or a lack of routine. (PC, ISSI)

Simply having children present at school was a priority since decisions made by parents or guardians for reasons not to attend were mostly likely due to health and aiga/whānau welfare.

Keeping track of absences and removing barriers to attendance was time-consuming and required more resources than Cathy and the school could provide. Cathy volunteered the school for a local project that aimed to raise both presence and achievement across the youth sector of Cedar. This project involved a wraparound service that included the MOE, public health nurse, and other principals in the cluster. Their efforts included sharing data, seeking common trends and sharing intervention responsibilities.

How

The who and what of NN3 were reorganised to focus on the disturbances and adjustments made and thus detailed how the NN moved. The main connection appeared as student attendance. The practice pattern was vulnerable when:

1. there was high student absenteeism.

The adjustments made in NN3 were as follows:

1. Cathy set a clear expectation of student attendance

2. Cathy became involved in a wider multi-disciplinary network, including health and welfare organisations, to monitor cluster-wide student attendance.

Cathy's principal leadership across NN3 set an example for how the NN and aiga/whānau would be supported to ensure their child's attendance at school.

WHY

NN3 perceived the rationale for interactions with Cathy as the development of a collective approach to wider schooling issues.

Cathy showed a willingness to take the lead in ways that buoyed not just Cedar, but other schools in the area. This was viewed as possible because of Cathy and the dedicated staff of Cedar. 
Nested Network 4 - Students

The purpose of NN4 was to form learning-focused, positive relationships with adults and peers in the school with the intention of developing key competencies and success in the NZC.

WHO

There were over 200 students in attendance ranging in age from 5 to 13 years. These students were part of the school as follows:

- Junior school whānau included students aged 5-7 years old

- Middle school whānau included students from 8-10 years old

- Senior school whānau included students from 11-13 years old.

Children from the middle school whānau, who ranged in age from 8-10 years (the same class of students who were involved in the Chromebooks trial) provided data about NN4. The student network was seen to establish connections with NN1, NN2, NN3 and NN5, NN6, NN7 and NN8.

WHAT

The main patterns of practice evident in NN4 included learning resources expectations, receiving feedback, and looking after the environment.

Firstly, students had a variety of resource expectations. They spoke of how much they loved going on trips, being given stationery and free fruit and milk. Students were especially complimentary about the Chromebook project which aimed to link student presence, engagement, and achievement. The Chromebook initiative was used by Cathy as a hook to guide the whole community into expectation of better educational outcomes. Students mentioned:

Our principal has meetings with the parents to talk about our work on Chromebooks. (CS, SA)

Cathy, however, noted an issue with an ICT-related curriculum:

The speed of change is driven by student competency. Kids themselves are demanding more change than what the staff can provide. (PC, FU3)

Here Cathy noted that adults might be slowing down student learning. During the student activity, students concurred, saying they wanted Cathy to get better at computers. 
Secondly, students communicated at length about receiving feedback on their work. Cathy was observed during Observation One responding to student work both face-toface and electronically. Students said:

Cathy shows us examples and evidence of what we are good at which is so helpful. She encourages us to take risks, talks to us about our work, gives ideas on what to achieve, tells us what to work on and tells us best steps to take. We talk about our learning all the time- we want to learn more. (CS, SA)

This is an example of moving students from strengths to next steps in a formative manner and suggests that students view learning discussions as essential. They also appear to have received the message that they needed to develop rigour. This is an indication of what Cathy referred to in her philosophy as a learner disposition.

Student behaviour was noted as a hindrance to the quality of student engagement. Students spoke of a strong desire to improve their behaviours and aimed to make Cedar bully free. Cathy supported them to change social behaviours:

She's around all the time .... She knows if there are fights and what we are doing. It's so we can get better at our work. (CS, SA)

Many students concurred that Cathy's monitoring of behaviour supported feelings of safety and their subsequent ability to refocus on improvement.

Cathy was observed to work directly with students at lunchtime as part of the school behaviour policy. This policy aimed to change abnormal behaviours, restore relationships, and develop personal competencies. The students who attended the group mentoring sessions were struggling to maintain expected behaviours, and Cathy supported them to think about ways to solve their problems by more appropriate means. Solutions were recorded and followed up by classroom teachers.

Thirdly, the environment was important to students and they felt it needed work as it was:

Untidy. We need a cleaner school that is rubbish free, spotless. It's better when it's clean. (CS, SA)

This quote demonstrates that the students noticed their environment and it impacted on their frame of mind. 
The student council ${ }^{39}$ was observed working with Cathy on ongoing environmental issues such as the development of a school garden and one representative was seen deciding which the tidiest class had been that week:

We give Miss ideas for a healthy environment. She talks directly to us and listens to our ideas. (CS, SA)

Cathy involved herself in working towards a pleasant school space. She took on small jobs and contracted out larger ones to professionals. Additionally, the school grounds closed at $5 \mathrm{pm}$ so that the opportunity of damage to school property was reduced. This often occurred at weekends, so a security guard was paid to protect the school.

How

Practice patterns were vulnerable when:

1. the ICT content/pedagogical knowledge of adults did not match that required by students

2. a student's ability to engage in constructive problem-solving was hindered by inappropriate social behaviours

3. some members of the wider school community did not support a safe and orderly environment.

The adjustments made in NN4 were as follows:

1. Cathy sought out more appropriate ICT expertise

2. Cathy worked to facilitate the improvement of behaviours with specific students

3. Cathy worked with students on environmental projects and contracted both paid and unpaid assistance to ensure a more pleasant school space.

Principal leadership in NN4 developed ICT knowledge, taught behaviours to assist with deeper learning, and worked collaboratively with students to both own and improve the school environment.

WHY

NN4 representatives perceived the meaning behind the network interactions as being about developing belonging and high expectations.

39 The student council is made up of middle-senior students who were responsible for sharing students' ideas, interests, and concerns with teachers and the school principal. 
Firstly, students talked about a feeling of belonging, reporting that they are an important part of the school. Furthermore, Cathy included the adults important in the students' lives. These included their parents and teachers from other schools.

Secondly, students reported that Cathy had high expectations of their learning:

She comes to see what we are doing. She encourages us and expects involvement in activities. As well, she encourages us to keep trying. (CS, SA)

Here it is evident that students' active involvement in learning is an important part of having high expectations. Some students even reported wanting to think like Cathy when they were older. This is consistent with the idea that educational success is about having a successful adult life.

\subsubsection{Network Two: Cedar Neighbourhood}

Network Cluster Two is the Cedar School Neighbourhood. This network includes interactions between Cathy and the school BOT, whānau/aiga (including parents), and school health and welfare services, all of whom are represented in the research. These members were sometimes, but not always, on site.

\section{Nested Network 5 - Governance}

NN5 had responsibility for the governance of the school and this includes its strategic direction and student achievement. As principal, Cathy took responsibility for the curriculum and school self-review process.

WHO

The membership of NN5 included:

- five elected parents (one of whom is the chairperson)

- an elected staff member and Principal Cathy as head of school.

All members provided data about NN5 and showed evidence of establishing connections with all other NN.

WHAT

The main patterns of practice evident in NN5 included monitoring and reporting on student achievement, discussing student concerns, and organising finances.

Firstly, the BOT communicated regarding the monitoring and reporting on student success. The observed meeting focused on writing and reading. Cathy spoke of the 
various assessments including e-asTTle writing ${ }^{40}$ and Standardized Test for the Assessment of Reading. ${ }^{41}$ Discussions focused on where big improvements had or had not been made and why. Overall it appeared that noticeable progress had been made but had not accelerated. Data hotspots of concern were honed-in on and included ESL students and a class of Year 3 boys. Concerns were expressed in the BOT meeting over the mismatch between the demands and expectations of the NS and the actual levels attained.

According to the principal's report, most students' literacy results were comparable or better than the NZ average but when scores in tests were aligned with NS a negative outcome resulted:

When the year 7-8 e-asTTle scores were aligned with NS expectations set by MOE, $64 \%$ of our students were below or well-below. There appears to be a misalignment between e-asTTle results and NS expectations. (PC, OB3)

There appears to be a suggestion from Cathy that Electronic, Aassessment Tools for Teaching and Learning (e asTTle) expectations in Year 7-8 were flawed. Cathy explained the tension in follow-up meeting four. She stated NS:

Positively assists teachers to report to parents, decide on next learning steps and create a type of urgency. (PC, FU4)

While this quote indicates positive aspects of NS, at a community and national level, normed tests used to make NS judgments may reinforce a false public belief that Cedar School students are not making adequate progress. This misalignment was of great concern to Cathy who had sent a letter to the MOE. Arrangements had been made to meet on this issue so that accurate measures of value-added progress could be assured.

Secondly, the BOT communicated about student environmental concerns. During Observation Three, the BOT were read a letter from the Hauora Team (student wellbeing) who expressed concern over the various sugary drinks being brought into school and requested they be banned altogether. By listening to student voice at a governance level, various school systems were to be adjusted to provide solutions, including a change to the school canteen items for sale and having the school caretaker

40 E-asTTle is an online assessment tool that assesses students' development in reading, writing and mathematics.

41 Standardized Test for the Assessment of Reading is designed to supplement the assessments that teachers make about their students' progress and achievement in reading. 
maintain the water fountains. Additionally, the BOT discussed other kinds of healthrelated topics. Emerging from this discussion was the need to insert an information sheet into the school enrolment pack as a way of reiterating for parents that some healthy food is provided free of charge.

Thirdly, the BOT communicated regarding finances. A recent financial report explored staffing, property, and learning opportunities. The financial report stated that the staffing budget was on track and in fact, in surplus. However, this surplus relied on the predicted school roll which was unstable, a trend Cathy reported as true for all schools in the Cedar area. An over-spend on staffing the previous year was approximately $\$ 35,000$. Cathy hoped the budget would recover but recovery was dependent on roll numbers increasing.

A tension related to staffing became evident when Cathy spoke about teachers' contractual entitlements. The Primary Teachers Collective Agreement gives a teacher a right, within reason, to be absent from school. During Observation Three Cathy was observed as saying:

\footnotetext{
The biggest overspend this year has been on relieving. Not much we can do about that I don't think. The Ministry gives us six days a year per teacher for relieving but the contract gives teachers a lot more than six days a year, including sick leave for children, which is totally appropriate but it does mean we go over every year. $(\mathrm{PC}, \mathrm{O} 3)$
}

Although teacher release is allowed for in the school budget, Cathy was expected to address the shortfall so that the budget was not exceeded.

Money spent on property was on the increase. Acts of vandalism had seen windows smashed and teachers' personal effects removed. In the year prior to the research, Cathy reported that the school spent $\$ 6,000-\$ 7,000$ addressing vandalism, which mostly occurred at night and at weekends. Vandalism appeared to be an ongoing issue. Taking photos of any community members defacing school property was suggested. Cathy sought continued support from the police, although BOT Member Three reported this was not always forthcoming. 
A tension existed between the longer-term property obligations of the 5YA plan ${ }^{42}$ and the speed at which these were being met by the MOE. The MOE was behind in approving the work identified by the school as being necessary. For example, the school hall required a new roof. This meant a more limited use of the school site, which was free of charge, by community groups. Cathy continued to follow up on progress and kept the BOT informed.

The money spent on learning opportunities was brought up by BOT Member Four, who felt holding parents more responsible for their contribution to their children's camp fee was needed. Approximately 12 families were identified as not having paid, costing the school $\$ 1,200$. BOT Member Four inquired into the possibility of recouping these costs. Although Cathy agreed that more assurance was needed that parents who could, should pay the fee, she also explained that there were some families for whom these expectations were impossible:

Some people have limited access to resources but still need a way for meeting needs and I have a way to give them an opportunity to break out of that. But it's perceived sometimes as a handout. (PC, FU4)

This quote suggests there is a community tension between people who contributed to school learning funds and those who did not. Cathy mentioned in follow-up meeting four that she thought seeing actions as attributable to one position or the other was perhaps due to Pacific village hierarchy where ways of behaving and thinking were more ordered. Parent Three comments:

\section{Her main thing is to make sure kids are at school. For me, because I have six, so Monday when it comes to Monday I don't even have enough, just maybe for the petrol and for my kids to go to kindy but for the one that comes here, go to school, go to school. (CCD, CSSI)}

This quote shows that Cathy placed schooling as a high priority, and was focused on having students attend school, rather than miss out on learning opportunities.

How

Practice patterns were vulnerable when:

1. nationally-normed tests were perceived as inaccurate

2. school structures did not support student health and wellbeing

42 The 5YA is a Five-Year Agreement fund that sets a minimum budget so that all schools can attend to any essential building work. Following certain rules, the BOT decides how to allocate the 5YA funding and accounts for this in the 10 Year Property Plan (10YPP). 
3. there was a budget over-spend on staffing and vandalism

4. property issues were addressed slowly by external bodies

5. social tensions existed between aiga/whānau.

The adjustments made in NN5 were as follows:

1. Cathy worked with the wider MOE to address what she perceived as discrepancies related to measures of success

2. Cathy encouraged students to communicate with BOT and parents on the topic of a healthy school environment

3. Cathy worked in partnership with teachers and community members to address a shortfall of finances

4. Cathy kept the BOT up-to-date and followed up with the MOE regarding building maintenance

5. Cathy focused on having students attend school, rather than miss out on learning opportunities.

Principal leadership in NN5 collaborated with the wider MOE on matters related to measuring student achievement, ensuring school structures matched health and wellbeing requirements, redistributing resources and addressing shortfalls, collaborating on more difficult principal tasks, and focusing on student presence.

\section{Nested Network 6-Aiga/whānau}

The purpose of NN6 was to develop mutually beneficial relationships around teaching and learning for students as well as to offer support to social and health networks as required. It was noted by the researcher that this network was not visible in the school on a day-to-day basis but was evident during less regular gatherings during a term.

WHO

Cedar School aiga /whānau were diverse and students may have had:

- parents from more than one ethnic group who may also be ESL

- a single parent or extended family networks

- parents and grandparents who attended the school

- both parents in the workforce

- access to the internet. 
Up to five families that met most of the descriptors above provided data about NN6 and showed evidence of establishing connections with NN1, NN2, NN3, NN4, NN5, NN7 and NN9.

\section{WHAT}

The main patterns of practice evident in NN6 included engagement, self-worth, and access to resources.

Firstly, the need for community engagement was communicated strongly throughout observations and interviews. According to Parent Three, Cathy took opportunities to interact personally with parents:

\footnotetext{
They had this kapa haka thing down at the arena last year and she offered to take myself and my two younger ones to go and watch the performance. We didn't have a vehicle and they were due to finish very late, like half- ten, eleven, but I wanted to see my son perform. The teacher and Cathy worked together to get myself and my two younger ones down there. In the car, she was talking to me not as a principal but as a person, about her own life. (CCA, CSSI)
}

This quote identifies what many parents felt, that Cathy was accessible to parents and connected in an authentic, compassionate manner. She, however, identified the need to strengthen communication with aiga/whānau on issues of academic success and wellbeing, stating that engagement was often sporadic. During the community interviews parents described Cathy as approachable:

If I didn't know Cathy that well I wouldn't be walking around here, I wouldn't be going in the staff room. I wouldn't have that free feeling to come around and just pop-in on the class where my baby is and speak with the teacher and all that. (CCF, CSSI)

This quote suggests that Cathy sets the scene for active participation by aiga/whānau which aligns with her philosophy of connectedness.

Student behaviour difficulties were topics noted as discussed one-on-one with families:

They automatically tell me what's happening. Cathy wants to solve the problem with me. For me to come in and talk about it, bring my son or my daughter. She listens to what the child says or feels. That's the thing because my son, he always in trouble. The person he wants to talk to is Cathy, to help him. (CCB, CSSI)

The example shows that Cathy engaged families and the student themselves in finding solutions to problems. She considered that her work involving whānau/families would never be finished, requiring ongoing conversations. 
Secondly, self-worth was communicated. Whilst students saw the Chromebook initiative as a tool which accelerated their learning, parents offered an alternative perspective:

I'm an Islander, I came from the Island, I know nothing, so pretty much I must start from the bottom. I don't expect my kids to have the laptop thing because of the stage I'm in. But because of her, she brings it in and all the kids can access it and they think they can do pretty much anything. (CCF, CSSI)

The Chromebook initiative was perceived by parents as working to develop their child's self-belief and achievement expectations. Cathy stated:

We do not make assumptions about how ethnicity, or home environment of any student may impact on learning. We instead believe in their potential to be successful learners, confident in their own identities. I can't leave, not until I have helped the community to see they have a great school and lots of good things happen here. (PC, FU8)

Here Cathy spoke of her long-term commitment to helping the aiga/whānau to reconceptualise the capabilities of their children, the school and the wider community. This strongly held belief led to a value placed on high expectations.

Thirdly, the availability of a variety of free and reasonably-priced resources and activities for families was communicated. Parents were entitled to use the school library free of charge and 'books in homes' provided new paperbacks for families to encourage reading at home. The school also made lunches each week for $\$ 10$ per child, if requested. This included healthy options such as a sandwich, yoghurt and fruit. Fresh vegetables were also provided for families who opted in. Parents stated:

\footnotetext{
There are lots of practical things going on for both families and students. Because we used to have breakfast here at school, now that was a success as well, she's making it easier for families, you know what I mean, just to make living affordable for all families. (CCA, CSSI)
}

This quote, indicative of many aiga/whānau participants, suggests that Cathy was viewed by aiga/whānau as helping to provide resources for day to day living. Parent B stated:

I think, ultimately her goal is the kids. She focuses on them as priority but we as the parents get the overflow from that. We benefit from what our kids are getting, and that's extremely unusual to see. (CCB, CSSI)

Here the idea was that, by helping students, Cathy also helped families. This is illustrated in her creation of an equity grant to help families financially support their children and is clear support of the school goal to develop mutually beneficial partnerships between the school and its community. 
Resources/activities were a source of tension for parents. In interviews, aiga/whānau mentioned Cathy's flexibility over their contributions and payment deadlines. For some families, extra contributions were impossible and in such cases Cathy accessed the school equity grant, although she stated that:

Deciding who gets the money, even with a criterion, it's difficult. (PC, FU3)

This perhaps suggests that making sure that available resources were directed appropriately was often challenging.

How

Practice patterns were vulnerable when:

1. there was an inconsistent learning relationship between home and school

2. parents had limited confidence that their children could succeed

3. aiga/whānau experienced difficulties in meeting school and home costs.

The adjustments made in NN6 were as follows:

1. Cathy made herself accessible to parents and interacted in an authentic, compassionate manner. Cathy additionally set an expectation for how the school welcomed its aiga/whānau members

2. Cathy empowered families to change narratives to develop a more positive perception of student learning outcomes

3. Cathy considered the implications of family and community financial difficulties.

She also used the school equity grant to further assist where necessary.

Principal leadership in NN6 gave parents a voice and kept communication open and personal with families, challenged parental expectations of their children and ensured that financial barriers to having children present and engaged at school were removed.

WHY

NN6 representatives perceived the meaning behind the network interactions as being about developing high expectations, respectfulness and practical support. Firstly, everyone strived to achieve at the highest level. A parent stated:

When you live around here it's that stigma, you must be dumb, you know. But she brings people together. All the kids think they can do pretty much anything, you know, they can do it. It's not a limit anymore, not a limit. (CCB, CSSI)

This statement is consistent with Cathy's understanding of the bigger picture and her desire for greater social justice. 
Secondly, Cathy was seen to be respectful of the community and its many cultures. Phrases such as "she accepts us", "lets us speak our language" and "celebrates us" were expressed by parents. The whole school was often referred to as "friendly", "familiar" or "like family". Cathy herself stated:

I'm not aiming to correct differences as deficiencies but rather to work towards a system that makes way for difference as a thing of value. (PC, FU5)

Such phrases and comments seem to suggest that the school culture is consistent with Cathy's dislike of deficit thinking and the desire to alternatively support a place of belonging.

Thirdly, Cathy was supportive. How she listened to parents was mentioned by Parent Seven who never felt afraid to talk with her because of her ability to notice what was going on at the school:

She just listens to us, she doesn't have an attitude and she's not forceful or aggressive. She's not just the principal. She is a principal, a teacher, you know, a mother, a friend. But everything is the same. She cares. (CCB, CSSI)

Cathy's ability to be herself appeared to be viewed by a number of parents as authentic and equalising. This quote suggests that Cathy was accessible to aiga/whānau.

\section{Nested Network 7 - Education support workers}

The purpose of NN7 was to work with the school to address the challenges that impacted students and their families and provided contacts for families to access resources in support of student success. This group reportedly expanded and contracted according to the relationships established by Cathy and the strength of these relationships.

WHO

The membership of NN7 included:

- Resource Teacher Learning and Behaviour

- a senior advisor from the MOE

- cluster colleagues

- university researchers

- virtual learning networks.

Cathy provided data about NN7. This NN showed evidence of establishing connections with NN1, NN2, NN3, NN4, NN5, NN6 and NN8. 
WHAT

The main pattern of practice evident in NN7 was the sharing of learning and development.

Cathy was an active member of a peer learning group. She provided support for newly qualified principals through a mentoring programme and was actively involved in cluster-wide meetings. Peer learning meetings were made up of educational leaders who aimed to share strengths and next steps for greater student presence, cluster-wide achievement goals, and management of PLD. The cluster had initially been without a clear vision. A shared mission had been set down with the help of an outside facilitator. As a result, a plan was being developed to address presence, participation, and achievement.

Peer support was sometimes lacking. A concern voiced by Cathy in follow-up meeting two was that a principal colleague was offering to bus children out of zone into their school. This would likely mean more local schools, including Cedar, may not experience an expected roll growth.

Wider support of Cathy was also evident in this network. Cathy made regular contact throughout the day with an MOE advisor on matters related to behaviour and staffing. Cathy gave several examples of how the senior advisor supported her:

\section{Dealing with staffing dilemmas and school financial concerns, helping to set up a transition programme between schools in the area, brokering the school's involvement in wider regional/national projects and, more recently, helping to set up the community of learning network. (PC, FU3)}

Here, the evidence suggests that the advisor was instrumental in supporting Cathy rather than managing her and supporting projects which would help sustain her own learning. This appeared to be helping Cathy to develop a wider and more dynamic NN7 to deal with the complexity of the work.

How

Practice patterns were vulnerable when:

1. there was a lack of shared vision

2. the behaviour of an individual cluster colleague worked more for the individual good of their school than the cluster as a collective

3. there were shifting priorities between compliance requirements and learning. 
The adjustments made in NN7 were as follows:

1. Cathy concentrated on what brought her principal colleagues together

2. An outside facilitator was brought on board for the cluster and a shared vision developed

3. Cathy's strong relationship with the MOE enabled her own learning needs to be prioritised.

Principal leadership in NN8 brought a wider group of learners together with a shared purpose, widened the NN, offered Cathy much needed support, and recognised the complexity of the work of the principal leader.

Nested Network 8 - Health and welfare services

The purpose of NN8 was to provide further contacts for families to enable access to what they needed to support their children's health and wellbeing. Shared between approximately five other schools in the area, the health and welfare workers were not on site every day but were present periodically throughout the week or term, as availability would allow.

WHO

The membership of NN8 included:

- Social Worker in Schools (SWIS)

- dental therapist

- school health nurse

- police youth aid officer.

The school health nurse and SWIS provided data about NN8 and showed evidence of establishing connections with NN1, NN2, NN3, NN4, NN5 and NN7.

WHAT

The main pattern of practice evident in NN8 was concern for aiga/whānau health and wellness.

The school noticeboard displayed a plethora of information on topics such as nutrition, the availability of counselling services and the implementation of special programmes such as a probiotics programmes. The school nurse and SWIS both made special mention of working with Cathy to bring parents on board with initiatives regarding emotional and physical health. Cathy stated: 
Many families who are renting are living in $\$ 300$ a week badly looked after, run down houses, without insulation and heat pumps. (PC, FU2)

This quote identifies that living arrangements were considered to contribute to the emotional and physical health of students and their families. Where possible, the SWIS worked with parents to get them into insulated homes with heat pumps and aligns with Cathy's beliefs about an awareness of the bigger picture.

Difficulties engaging parents in dental health issues was experienced by the health nurse. Advice was sought from Cathy, who made suggestions which included working with a cultural advisor on effective ways to initiate contact with aiga/whānau. Both the SWIS and the health nurse also mentioned the formation of a multi-disciplinary team. Together, these same teams problem-solved student and whānau issues. Cathy confirmed that:

Meetings about priority kids takes a lot of my day. (PC, FU2)

This quote suggests that the wellness of particularly concerning children was a priority for Cathy and often included aiga/whānau considerations.

How

Practice patterns were vulnerable when:

1. living standards were precarious

2. there was difficulty initiating contact with parents

3. there was difficulty getting available resources to where they were most needed. The adjustments made in NN8 were as follows:

1. Cathy emerged a new transdisciplinary approach to address aiga/whānau needs

2. health care professionals and Cathy called on the wider community for cultural support.

Principal leadership in NN8 made connections with the wider network for support, resourcing and cultural backing.

WHY

NN8 representatives perceived the meaning behind the network interactions as being about developing inclusion, advocacy, and navigating wider social problems.

Firstly, those in the health and welfare services were embraced as having a place at Cedar. Cathy was viewed as welcoming, always opening the school door to them: 
This quote is indicative of many community members who felt included in the school community and were thus more likely to make supplementary visits.

Secondly, Cathy was perceived as an advocate. The health and welfare representative mentioned her ability to both listen to what was needed and attain resources. She advocated for teachers, students and families.

Thirdly, being able to navigate wider social problems was noted. The school social worker suggested that Cathy saw the bigger picture as impacting a student's learning rate and had:

An eye for finding gaps between disciplines and connecting these up. She'll think of people we need there and email straightaway. (CCG, C1SSI)

This quote suggests that by building relationships between people in the community, Cathy used a more systemic approach to influence student learning.

\subsubsection{Network Three: Wider Cedar Community}

Network Three is representative of the wider community and includes NN9.

Nested Network 9 - Private businesses and organisations

The purpose of NN9 was to support initiatives that helped reduce inequity in the area. It appeared that the membership of this group was reliant on relationships Cathy had established.

WHO

The membership of NN9 included:

- local businesses such as a bank and tree felling services

- various trusts of which three were mentioned in the interviews.

The trustee of the Chromebook initiative and Cathy provided data about NN9. This NN showed evidence of establishing connections with NN1, NN5 and NN6.

\section{WHAT}

The main pattern of practice evident in NN9 was accessing otherwise unobtainable resources.

Applying to external trusts was one of the main ways that the school accessed otherwise unobtainable resources and a means to ensure innovative use of ICT. During follow-up meeting one, Cathy mentioned a variety of trusts that funded the technology 
and music curriculums. The ICT-focused trust provided Chromebooks for students and was being trialled in two classes. The school took this on with just three other schools in the area but the membership of this initiative continued to grow. At the time of this research the Chromebook Trial included eight local schools:

Cathy willingly worked with me to communicate with families how the initiative works, and how it might make a difference to student learning. (CCH, C1SSI)

Cathy understood the importance of new and improved resources and the need to involve aiga/whānau in the initiative. The Trust representative noted the strength of relationships as vital to keeping initiatives such as this going. Cathy, additionally, looked to a university colleague in NN8 to see if research could be done to ascertain whether in fact the Chromebook initiative had inadvertently improved home-school relationships:

\section{When you think about the collective impact thing ... drawing on people from across the community; private businesses and others ... these people can be quite influential for learning. (PC, CISSI)}

This quote suggests that Cathy not only sourced resources in new ways but looked to connect the resource providers so that influences on learning could be measured and sustained. This NN relied heavily on Cathy.

How

Practice patterns were vulnerable when:

1. parents were not considered in the innovation

2. there was a reliance on Cathy to establish NN9 connections.

The adjustments made in NN9 were as follows:

1. Cathy engaged aiga/whānau in any new initiatives

2. Cathy sought support from research to see if, in fact, the investment of time spent working with trusts to engage whānau had a worthwhile effect.

Principal leadership in NN9 refocused efforts on the effects new resources had on both students and whānau.

WHY

The NN9 representative perceived the meaning behind the network interactions as being about developing equity and social justice, especially for minority students from Māori and Pasifika backgrounds. 


\subsection{Change trajectory}

Examples of the types of changes occurring and how Cathy's principal leadership network self-organised to maintain and progress itself when disturbed, became evident and can be seen below in Table 13 .

Table 13: The feedback loops occurring in Cathy's principal leadership network

\begin{tabular}{|c|c|c|c|c|}
\hline NN & Cyclical feedback loops & $\begin{array}{l}\text { Developmental feedback } \\
\text { loops }\end{array}$ & Feedback loops ignored & Possibility of emergence \\
\hline 1 & Equal & Equal & & Evident \\
\hline 2 & Mainly & Yes & Yes & Possible \\
\hline 3 & Equal & Equal & & Possible \\
\hline 4 & Equal & Equal & & Possible \\
\hline 5 & Mainly & Yes & & Possible \\
\hline 6 & Mainly & Yes & & Possible \\
\hline 7 & Equal & Equal & & Possible \\
\hline 8 & Equal & Equal & Yes & Evident \\
\hline 9 & Equal & Equal & & Possible \\
\hline
\end{tabular}

Cycle feedback loops worked to maintain status quo and were evident in NN2, NN5, NN6. These feedback loops successfully addressed issues with: home-school relations, finances and building maintenance, wellbeing, school image, student expectations, assessment, and competition. Day-to-day, these areas appeared most problematic.

Developmental feedback loops were further evident across the entire principal leadership network meaning the possibility of emergence was spread throughout. This suggests that the energy and time given to making adaptations and change was of benefit.

There was a balance between both cyclical and developmental feedback loops in NN1, NN3, NN4, NN7, NN8, NN9 suggesting actions and behaviours were having a balanced impact on change.

There was evidence of ignoring of feedback in NN2 and NN8. This occurred mainly with teachers in relation to pedagogical development and with aiga/whānau in relation to developing home-school relations. 
There were two examples of emergence in evidence in NN1 and NN8. This occurred mainly with teachers in relation to school organisation and with aiga/whānau welfare needs. Student, teacher and aiga/whānau wellbeing were prioritised equally.

Overall Cathy's principal leadership trajectory is not regressing but progressing and mainly developing. The possibility of transformation in support of more extensive educational reform is possible.

\subsection{Chapter summary}

Principal leadership practice at Cedar can be viewed as both a collective and individual construct. Both are summarised here to show the culture of the network.

\subsubsection{The principal leadership network}

The collective construct of principal leadership is bounded by shared relationships, understandings of educational success, and collective practices. Education success was perceived by the Cedar principal leadership network as developing learners with a strong identity and confidence who engaged in ongoing learning opportunities. These learning opportunities wove together social, academic, sporting, and cultural aspects of the individual but were also for the benefit of the collective. Achievement may have been measurable, obvious and extrinsic, as well as less obvious, immeasurable, and largely intrinsic. The daily principal leadership practice patterns were centred around: the delivery of a holistic curriculum; PLD for all staff inclusive of the principal; engagement in the learning process, community learning and wellbeing; and formative and evaluative assessment; and resourcing.

Practices were disrupted by: PLD opportunities and resources that did not meet teacher learner needs; limited teacher knowledge and pedagogy in areas of ICT and science; the perception of testing inaccuracies; the speed at which solutions to more complex learning problems could be found; high student absenteeism and inappropriate social behaviours; an unreliable learning relationship between home and school; getting resources to where they were most needed; tensions between aiga/whānau; a mismatch between the public and private views of educational success; a misuse of school grounds; parental views on achievement possibilities; and finding positive housing solutions for individual aiga/whānau. 


\subsubsection{Principal Cathy}

Principal Cathy had developed a desire to "break the cycle" of poverty. She had a strong sense of where she had come from, and had also developed a confidence to challenge the status quo. Cathy strove to create a safe environment where hard questions could be asked so teachers rose to the challenge rather than resisted it. This denoted a move away from engaging for compliance towards a desire to engage for need. Others' perceptions aligned with this philosophy and included being futurefocused, using a collective approach that advocates for equity and social justice, developing belonging for all, creating a space in which a respectful, practical and supportive cross-disciplinary approach is used, and finally, ensuring learning conversations, where knowledge, challenge, and high expectations are generated.

As the network emerged and self-regulated, Cathy showed strong evidence of collaborating on some ideas, challenging, and attuning her ideas and actions. She developed a collaborative inclusive culture within the school and beyond. Collaboration was especially evident with aiga/whānau and health care professionals and occurred with multiple groups and for multiple reasons. The reasons for collaboration included: to share resources; encourage problem solving; share an understanding of what learning looks like, offer cultural support; develop flexible authentic, compassionate relationships, and improve the physical environment. Cathy spent a lot of time challenging staff, BOT, aiga/whānau and politicians by pushing forward with initiatives, and working to change the narrative around school attendance and the investment of aiga/whānau in school life. Cathy also spent time attuning ideas and actions. She regularly used strong relationships to resolve issues of concern. For example, Cathy balanced the diverse requests made by families with a fair distribution of additional resources to ensure school presence and participation. When developing the school budget, Cathy developed a strategic plan to offset the lack of timely and appropriate resources. To rebalance the public perception of the school, Cathy encouraged families to change narratives to develop a more positive parental perception of their child's/children's future selves. Finally, Cathy worked to balance complexity with progress by accepting teaching and learning as being difficult. She accessed mentors, had high expectations, and developed both a widened curriculum and plans for assessment that showed progress. 
The collaborating, challenging and balancing of ideas helped the network adjust so that three main trajectories could be maintained: developing a learning environment that was functioning, accessible, safe and orderly; an engagement in learning for both students and teacher with clear shared goals between the two; and the development of community-school partnerships that focused on health, wellbeing and open communications with aiga/whānau.

\subsubsection{Network capability}

The movement of Principal Cathy's leadership patterns of practice at Cedar School tended equally towards developmental change, with evidence of emergence and potential for still more. While disturbances were regular, the internal network could self-regulate so that teaching and learning focused work could continue. The creation of new structures in support of connectedness and cohesion, and Cathy's focus on these two attributes, is more than likely one reason for this. Whether the changes identified are sufficient to withstand ongoing disruptions is unknown, as is the types of emergences that might increase the likelihood of transformational change across the wider education system. 


\section{CHAPTER 7 \\ Exploring principal leadership practice}

"Tyranny is the deliberate removal of nuance" Albert Maysles

\subsection{Chapter overview}

The two previous chapters described what the phenomenon of principal leadership in a LSE setting looked and sounded like in practical terms. Bronfenbrenner's (1979) ecological systems theory was used to identify boundaries and to organise the data into levels. Examples of principal leadership practice patterns occurring in each of the levels were described, using an adapted version of Capra's (1997) scientific understanding of living systems. How patterns were enacted, made vulnerable, and readjusted, exposed feedback loops of various kinds. In this chapter, the overall goal of the living system is first explained. The trajectory of success is detailed. Then, the two units of analysis - the two principals and their contexts - are combined. Being in leadership had a focus on systemic movement towards success in context and allows for an exploration of the important commonalities and differences pertaining to the inner mechanisms of principal leadership practice patterns. Support mechanisms that have the potential to reduce constraints and increase momentum are identified, as are those that increase constraints and reduce momentum. By shifting thinking from seeing parts to seeing the whole (Senge, 2006) the capability of the system overall to self-regulate is investigated. The chapter concludes with a summary of the relationships among the features of the case study.

\subsection{Exploring the trajectory of educational success}

The principal leadership networks influenced positive movement towards educational success. Therefore, how participants in this study understood educational success is important to discuss. Participants were focused on the development of the whole person, on learning as a process, and on ecological wellbeing, and shows what Fullan (2011) refers to as a breadth of purpose.

First, educational success was inclusive of the many aspects of self. While recording cognitive successes in literacy and numeracy, both Amber and Cathy's networks additionally took the opportunity to validate students' social, physical and cultural 
talents via the school newsletters and at school assemblies. Moreover, the Acacia curriculum was influenced by the Pasifika and Catholic ways of being and the Cedar curriculum, the Pasifika and Maori ways of being. My findings indicate that both principal networks, rather than continuing to legitimise a narrow success agenda (Snyder, 2013), were working to broaden the curriculum, even validating the influence of culture on the local curriculum. This is more in line with work in countries such as Canada (People for Education, 2013), parts of California (Krachman, Arnold, \& Larocca, 2016) and Finland (Sahlberg, 2018) where the focus is on developing a wellrounded individual. The research found that the network acted to broaden the curriculum in a way that cultivated the whole child. A greater diversity of talents, successes and identities, especially those related to culture, need to be recognised alongside literary and numeracy success measures.

Second, success was viewed as a process of engagement. Cathy monitored individual student work electronically to ensure reflections, next steps and improvements were recorded. These finding aligns with recent research by the NZ children's commission (2018) and work by Wheatley in Frieze (2010) who argue that understanding goals and working with success as a process helps to provide greater student autonomy over learning as well as tighter measures on how students are performing. Amber's network, while also monitoring progress, included student portfolios to simply show success in less traditional areas such as attitudes to learning. These are what Claxton et al., (2016) and Zepke and Leach (2010) refer to as soft skills. Educational success in this research was inclusive of developing an understanding of improvement as 'value-added'. More personalised formative measures of success need to be established alongside collective, summative measures.

Third, success was viewed as progressing social and environmental responsibility. Amber aimed to support the community to develop respect for self, others, and the environment. Correspondingly, Cathy had co-constructed a strong concern for the local environment. Success is therefore deeply ecological (Capra, 2013). A type of growth that is ethically just, focusing not just on wellbeing and success of students 
now, as suggested in the Tomorrow's schools review (MOE, 2018), but that is focused on a collective response to future social and environmental wellbeing needs.

\subsection{Exploring patterns of practice}

Over many months of data collection and analysis, four broad patterns of principal leadership practice emerged that moved the learning community towards a more holistic curriculum, on learning as a process of engagement and adding-value, and on developing an awareness of social and environmental responsibilities. The patterns are shown in Figure 10 as - building the network; integrating multiple perspectives; facilitating cohesion; and confronting and addressing injustices - with each pattern linked intrinsically to the others.

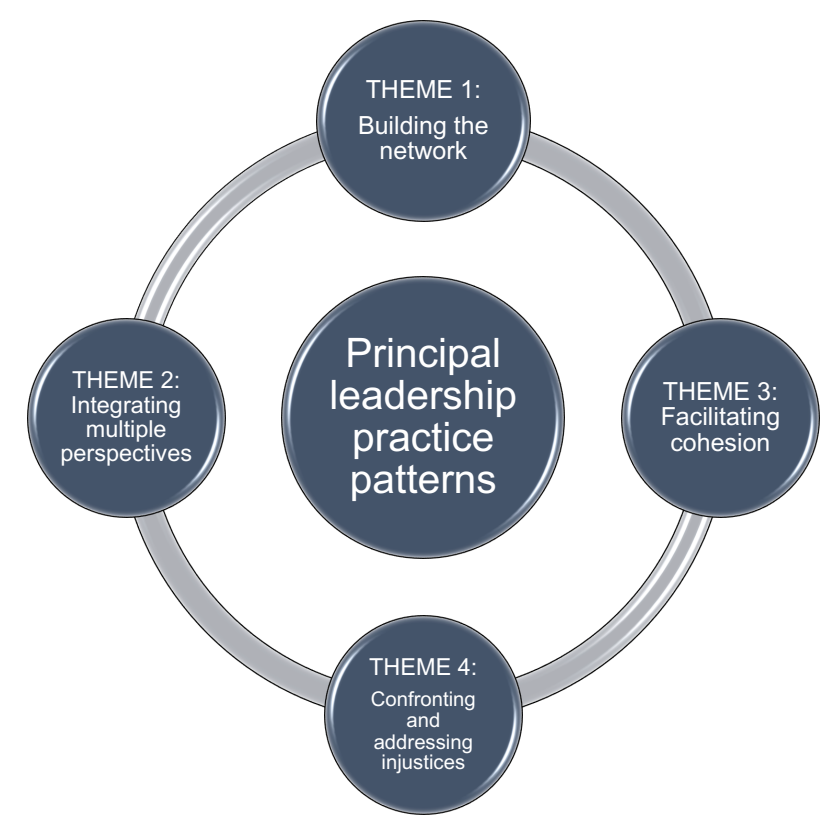

Figure 10: Exploring the principal leadership patterns of practice

When viewed as a complex system these broad patterns are the most salient way of exploring how principal leadership practice took place in context for improved educational success.

\subsubsection{Theme 1 - Building the network}

Theme 1 explores the shape and boundary of the LSE principal leadership networks. This theme links to the 'building relationships' practice pattern identified at the end of the literature review. The review concluded a need for leaders to develop interactions that are strong and trusting (Agosoto \& Roland, 2018; Giles \& Palmer, 2015; Bryk et al.2015). This section discusses how the networks built relationships in practice. In 
particular, how they created stability, developed links to a diversity of active members, developed interconnections between middle leaders and students, focused on the minimally engaged as well as those with an especially positive influence, and developed new and unexpected connections.

The principal leaders' connections encouraged network stability. Amber had been at Acacia School approximately nine years at the time of the research and had attended the school as a child. Cathy's tenure at Cedar, including her time as a relief teacher and deputy principal, exceeded 13 years. Parents and staff viewed both principals as having an ongoing positive influence in the community. The findings suggest that principal leaders in this study used the historical and often tacit knowledge of their respective communities to advance their leadership trajectory. These pre-established connections are referred to by Wilson et al. (2017) as belonging in 'place' and inclusive of sensibility or emotional attachment. The depth of this belonging may have helped these LSE principal leaders to advance a greater sense of followership, especially by teachers. A leader's long-standing connection to the place in which they practise, places confidence in the network from within and offers a way to increase systemic leverage over time.

Each leadership network was made up of diverse, active daily members including teachers, in-school support staff, and BOT and education support workers. All were seen taking an active part in delivering the school vision. Interactions had varied purposes and related to attendance, behaviour, ensuring achievement outcomes, or worked to support school frameworks and policies, with classroom practice, and planned PLD. Often interactions identified the structural changes required. For example, a principal visit to parents was deemed important by teachers in support of greater student attendance. Moreover, a restorative justice programme was encouraged by the MOE to help improve student behaviour and was implemented by the staff and monitored by the BOT. Participation by a diversity of network members, requires links to be made between leadership and learning (Dufour \& Marzano, 2011; Robinson et al., 2009) with more recent literature focusing on the coming together of leadership and organisational governance (MOE, 2018). Relationships connect teaching and learning, leadership and governance so structural changes in support of increased engagement can be more easily made. 
The day to day network connections relied especially on middle leader engagement. At Acacia, the leadership team kept aiga/whānau connected to schooling, worked to maintain ICT resources, and monitored teacher quality. At Cedar, the middle leaders supported Cathy to develop the curriculum, teacher PLD opportunities, and school organisational structures and solve complex learning dilemmas. More evident at Cedar was actions focused on growing pedagogy and resources rather than simply maintaining them. While findings show that all middle leaders were involved in the expected pedagogy and management (Walker et al., 2008) and the flatter type leadership advocated for by Harris (2009), the negative impact of unstable aiga/whānau - school relationships and the leadership team's efficiency, was evident at Acacia. Here the formal leadership team focused on maintenance of relationships, so that pedagogical advancement opportunities were maintained as much as possible.

Each principal noticed a need for greater interconnectedness between students and their school. Amber took opportunities early on in her tenure to change structures so that student learning was recorded more fully, and in a way that students could easily share with their parents. The students at Cedar participated in influencing their health and wellbeing, working with the BOT to implement healthy eating plans, policy changes in support of improving their health, and communicating clearly with aiga/whānau about the food available both free of charge and at a reduced rate. This finding suggests that the principal networks developed the ability of students to influence their learning environment. Ferriter and Cancellieri (2017) maintain that working to empower students rather than simply engaging them, is one of seven practices that assist principals in creating greater cohesion across their work (Wylie, 2017). Strengthening student voice, saw students have more say over their learning context.

The networks focused on the minimally engaged. While a BOT was a staple at both sites, it was challenging to secure parental representatives at Acacia. One parent representative on the Acacia BOT was seconded but as reported by this same member, juggling work commitments and childcare had made seeing out the three-year tenure impossible for most. Additionally, Cathy engaged a private contractor at extra cost to the school, to undertake the property and finance portfolios, admitting these skills were beyond the capabilities/interest of the current BOT members. The Tomorrow's Schools policy, established in 1989, saw the diversity of skills, beliefs, and knowledge 
of their community become a valued part of the school decision-making process. However, in the LSE setting, the operational and commercial skills that boards need are not as frequently found. This research highlights a social issue that cannot be overcome merely by seconding, supporting and retraining members, since the origin of the problem lies in broader considerations such as employment and child care. Wylie (2012) argued that finances and property were time-consuming for principals in the initial years of Tomorrow's Schools. Nearly 30 years on, my research suggests that in smaller LSE schools, this is still a significant problem. LSE school governance relationships require a lot of principal time, and continually draws focus away from teaching and learning.

Education community members positively influenced the principal leadership networks. Amber was highly involved in the Catholic principals' network while Cathy was also central to the local community of schools' network. Cathy also worked to establish and sustain school-university collaborations. Collaborations instigated first by the university, considered the use of Chromebooks in student learning which later evolved, at Cathy's request, into a co-constructed investigation into the impact this innovation was having on home-school partnerships. In this instance, learning is promoted as a school-led endeavour that is supported by the wider learning community. Caldwell (2005) refers to this learning scenario as the lateral transfer of knowledge. To secure a diversity of stakeholder involvement however, one way of being and knowing, should not be privileged over another (Caldwell, 2005). Lateral transfer of knowledge may be especially useful in bringing theory-based institutions (universities, for example) closer to the action of learning to explore complex learning issues and help improve achievement results in schools (Walker et al., 2008). The education community members helped to bridge the divide between theory and practice.

The network sought varied connections to support learning needs. Amber made serendipitous connections with community outreach groups as opportunities arose and commented that a rationale for the connections could not always be predicted but once made, they were likely to be beneficial in some way. Cathy drew in outsiders such as politicians and local councillors with a specific purpose in mind. Observed reasons for selection included to provide resources and encourage a community spirit. Relationships formed in the LSE context may or may not be deliberately strategic, and 
yet could still be beneficial, making social networking an essential skill for principal leaders. Stone and the Centre for Ecoliteracy (2009) specifically refer to this as working simultaneously at several levels of scale and Ovens and Godber (2012), instigating affordance networking. In this study working simultaneously meant that which helped create both serendipitous and purposeful relationships, for both expected and unexpected possibilities and resources. Principal practice developed ordered and disordered connections which would help to meet present and future needs.

The networks included unexpected members at the periphery. Through Amber's connectedness, a 'Violins in School's Music Trust' sought to create opportunities, connect communities, and nurture future leaders through the provision of free music lessons and performance opportunities in support of the school goal related to the education and development of the whole child. At Cedar, Cathy contacted a trust to harness opportunities offered by new knowledge and technologies to secure a sustainable social, cultural and economic and environmental future for her students. The presence of business and private organisations Cathy's willingness especially, to diversify membership beyond the usual education-related participants, to improve social cohesion and resources, or "to seize opportunities to build positive social trends within the microcosm of schools" (Smith, 1988, p. 228). As appears obvious here, schools are no longer silos and apart from the community (Shields, 2013) but can be expected to combine with other groups for greater collective impact (Kania \& Kramer, 2011; Smart, 2017) and show evidence of an active ${ }^{43}$ network. Widening network boundaries ensured that a greater variety of relationships in support of learning excellence and social cohesion were possible.

This section discussed the membership and purpose of the principal leadership network. To reduce the constraints to the principal leadership practice pattern, both networks provided substantial input into a diversity of relationships, where overlapping hierarchies worked to strongly connect with students, aiga/whānau and governors, but relied heavily on middle leaders. The network included a principal who had a strong sense of place and confidence in the network's ability to thrive. Principals

43 A system that is both open and closed is referred to by Capra (1997) as being active. A boundary open to new and useful energy entering the network while also keeping out negative or unproductive energy that threatens the identity of the network. 
developed ordered and disordered connections which saw the boundaries widen. This pattern connected learning excellence to social innovation.

\subsubsection{Theme 2 - Integrating multiple perspectives}

Theme 2 explores the culture of LSE principal leadership networks. The theme links to the 'integrating multiple perspectives' practice pattern outlined at the end of the literature review. The review identified the need for leaders to be responsive to the many traditions, rituals and beliefs that make up a community's guiding culture and described that which is ideally socially inclusive of all (Johnson, 2014; Santamaría \& Santamaría, 2016). This section discusses how the networks set about integrating multiple perspectives in practice. In particular, how the networks validated cultural uniqueness, encouraged multiple perspectives, and engendered a deep sense of 'family'. Conversely the network also identified deficit theorising and challenged negative points of view in the wider community.

The networks ensured the school's unique collective identity was validated. The school charter at Acacia, in keeping with being a Catholic school, highlighted spirituality and aimed to support the community to live the values of Jesus Christ to ensure the development of respect for self, others, and the environment. Conversely, Cathy had co-constructed a strong cultural identity with tangata whenua and a strong concern for the local environment. My research shows that the network is connected beyond superficial collective commonalities and brings the LSE leadership network into the realm of deep ecology (Capra \& Luisi, 2016). This refers to mindfulness (Cordon, et al., 2009) or mana wairua (spiritual and holistic wellbeing), where all living beings are members of ecological communities bound together in a network of interdependencies. Identity goes beyond the superficial, so that the schools' past, present and future ways of being are considered.

Differences were encouraged through a school learning culture aimed at working with and for diversity. Rather than compensating and correcting differences, both principals saw difference as a thing of value: languages other than English were used, principals dressed for special occasions in traditional costumes, and family groupings and cultural customs were incorporated into school structures. A teaching complement that considered ELL needs requires specific consideration. Amber included a part-time ELL teacher as the budget allowed, whereas Cathy employed parents as teacher aides 
to strengthen students in their first language. My research shows the efforts both principals made to address the needs of ESL students and in doing so, work to narrow what Tollefson and Magdaleno (2016) refer to as a culture gap. For additional consideration is the work of McGee et al. (2014), who argue that as the number of ELL's continue to grow in NZ schools, a strong focus on developing the capacity to support ESL teaching and learning is essential. Both principals valued difference enough to include dominant ethnic voices into the teaching and learning programme.

Each network's culture engendered a deeply rooted sense of 'family'. Amber stated that strong relationships were the foundation of her work and had stood the test of time. Cathy enjoyed interactions that ensured a school culture of 'love, belonging, and freedom'. My research reveals that in these LSE contexts, belonging is requisite for both students and adults, giving a deeper meaning to the work of schools. For principals in the LSE context, the need to build 'whānaungatanga' - an extended family-like relationship that is positive, meaningful and respectful (Hohepa \& Lloyd, 2008; MOE, 2010; Parrett \& Budge, 2013) - is a real need. Evidence suggests that while not sufficient alone, whānaungatanga is fundamental to higher cognitive demand and engagement (Bishop et al., 2013). The networks supported greater emotional stability of its members.

The principals in this study spoke clearly about the effects of in-school deficit theorising of school culture. The principal leaders reported having explicitly confronted the deficit thinking of staff from early on in their tenure. Amber used positivity to counteract deficit thinking, which she said tended to emerge during times of stress. Cath, however, firmly reiterated her belief with staff, refusing to accept home circumstances as a reason for underachievement. Teachers at Cedar appeared to have adopted the belief that deficit thinking was unacceptable. Shields (2013) and Parrett and Budge (2012) highlight such thinking as counterproductive, as well as the need to idenitfy the strategies and beliefs assumed by such thinking. In NZ however, Thrupp (2017) argues that attitudes and power relations that maintain deficit thinking have been largely ignored. Deficit thinking is being challenged by these two NZ principals, but maintaining the challenge requires ongoing energy and is therefore an ongoing issue. 
The principal leadership networks also developed an awareness of negative points of view that played out within the wider neighbourhood. While school charters, websites and official school pamphlets all helped foster a positive image, a mismatch existed between what the principal network saw as a highly effective, quality teaching and learning environment, and variable external views. Amber referred to families that moved out of the community or to another country entirely for a better life, but she had a clear vision of the successes that were occurring regardless. Cathy referred to 'brown flight,' a concept that suggested that the community responded to the deficit mentality by wanting to leave the LSE context entirely. Stroh (2015) states that leaders must often work with damaging views of schools and communities with Miller (2018) adding that this is evidence of the influence of national culture and context. A variable perhaps less considered in recent research is therefore the deficit theorising that occurs within the LSE community about itself. This finding challenges the work of Carpenter and Osborne (2014) who lean more towards deficit theorising coming from internal school viewpoints. By working with wider community views Cathy has a chance of widening her sphere of influence and producing more positive competitive value. Miller refers to this type of forced compliance with entrepreneurialism, as a practice that will likely become firmly embedded into a principal's work.

This section discusses the wellbeing of the principal leadership network. To reduce constraints to this principal leadership practice pattern, the network developed: a collective identity considerate of past, present and future; a curriculum inclusive of the dominant ethnicities of the community; members' emotional welfare; and a principals' sphere of influence. Social wellbeing was conversely obstructed by deficit thinking and the inability to evolve the learning culture due to financial constraints. This pattern connected learning excellence to the development of the school culture.

\subsubsection{Theme 3 - Facilitating cohesion}

Theme 3 explores the interdependent behaviours of the LSE principal leadership networks. This theme links to the 'facilitating cohesion' practice pattern outlined at the end of the literature review. The review identified a need for leaders to develop a cohesive community of practice for both survival of the network and the opportunity for it to prosper (Heck \& Weiss, 2005; Senge, 2006). How the networks managed interdependence in practice is discussed. In particular, how the networks developed 
physical and psychologically safe environments, encouraged collaboration and addressed inaction.

To facilitate cohesion, a safe environment was an ongoing consideration for both networks. The physical safety in both schools, despite support from neighbours, various procedural statements and the implementation of health and safety policies, still relied on security and remedial maintenance. Police were often called due to vandalism, theft, or risks to personal safety. My findings suggest a safe environment was of the utmost importance to the network. This finding exemplifies the work of Blackwell (cited in Gordon, Jean-Louis, \& Obiora, 2017) who suggests that a safe learning environment, with consideration for aspects of the wider community, is a fundamental requirement in the LSE context for promoting and improving student learning. A safe wider environment is additionally essential in support of a school's vision (Cotton, 2003; Jacobson, Brooks, Giles, Johnson, \& Ylimaki, 2007; O’Donoghue \& Clarke, 2009) since unpredictable stress is also demonstrated by Yang (2014) to impair the brain's capacity to study and retain information. The school environment therefore impacts on a student's cognitive progress. Robinson et al. (2009) conversely argue that leadership practices that create an orderly and supportive environment need only focus on the management of students within the school. They also argue that orderly and supportive environments have minimal effect on learning. To the contrary, my study lends support for the importance of safety of students, the school, and its neighbourhood and may be evidence of what Parrett and Budge (2012) in fact refer to as evidence of a focus on systems learning. A safe environment helps the network reduce stressors, so that interconnectedness remains focused on learning.

The psychological safety of network members was additionally important. To address bullying and aggressive behaviours between students, a variety of school-wide positive behaviour for learning programmes and restorative practices were introduced. Both principals spoke directly with parents and relayed information regarding the home situation to teachers. My research indicates that a role of the principal leader is to garner support for improved in-school behaviours alongside families, so that home levers for behavioural change are better understood. This finding supports work by Parrett and Budge (2012) who suggest that becoming a safe school requires structures and processes that address inappropriate behaviours, but that also create connectedness between the school and the students. Establishing strong interconnectedness with 
families regarding student behaviour, means there is likely to be reduced disruptions to engagement in learning.

Collaboration was used as a main structure for ensuring connectedness. Amber and Cathy focused on shared or distributed actions, shared planning and programming, and co-developed resources and responsibilities. Moreover, when the unexpected occurred, for example when a staff member's health created a lengthy absence, or staffing needed to be reduced, the connections already established were strong enough to adapt to the added pressure. This research finding shows what Spillane (2006) refers to as a stretching of leadership and is empirical evidence in support of Klimek et al's.(2008) claim that through collaboration, the organisation is more likely to have the commitment and energy to sustain it through difficult periods. Collaboration was emphasised as a way of sharing power more fluidly between the network members, especially during times of unexpected pressure and stress.

The principals used the art of conversations as a powerful tool for collaboration. Staff and parents at both Acacia and Cedar were motivated by a principal who was open to discussing new ideas, and they spent time working alongside the principal to find shared solutions and actions. While various communication structures were well established, my findings suggest that the art of conversation was a deceptively simple strategy used by these principal leaders to prompt a shift in thinking about who can learn and who can lead. Leadership practice became a fluid interaction that offered an opportunity for wider network growth. Waheed et al. (2018) refer to the fluidity between leading and learning as enabling, and Capra (1997) and Raelin (2016) as a form of empowerment. Raelin specifically states power can be "released through conversation" (p. 2). My findings suggest that by involving the network members in conversation, the network was more inclined to act. Empowering others through conversation was, however, time-consuming and might quickly become a lost change opportunity.

Additionally, where solutions to problems were not immediately forthcoming, the principal relieved stress using humour to maintain a positive tenor and the engagement of others. Hurren (2006) found that humour can facilitate collective wellbeing while mandating the space for contextual understanding. In an LSE context where change is constant, principals require various personal attributes to keep the network connected. 
Shared humour should not be overlooked as a successful way of keeping opposing viewpoints united.

Reasons for aiga/whānau inactivity was addressed by the principal leader. At Acacia, staff were cynical about their lack of engagement in school life. Amber determined the reason for poor engagement was not because parents did not care but instead looked for a more culturally considerate alternative. While noticing is essential, the research also implies that carefully choosing what to ignore is also essential. Findings suggest that principal practice included mindfully ignoring some concerns, so that reframing problems and sourcing alternative solutions became possible. Ignoring teacher concerns challenges the work of Day (2017) who states that ignoring challenges may threaten effective practices. Along these same lines, Hoppey and McLeskey (2013) state the role of the principal is to focus on providing support for teachers to do their best work. My finding aligns with Shields' (2013) work, which suggests ignoring concerns to explore problems more thoroughly and finding alternative solutions is preferable. By choosing to remain connected to student and aiga/whānau learning and wellbeing needs, interconnections between home and school were positively maintained.

Positive levers for engaging parents in school life were in fact, developed. Amber registered for the Talanoa project, a research-based initiative that encouraged the school's parents to lead other parents, combining the project with 'Computers in Homes', an initiative focused on providing parents with a computer and developing their ICT skills. At Cedar, aiga/whānau were invited to celebrate culture concurrently with curriculum discussion. Additionally, a Chromebook initiative was started. Both principals saw the benefit of harnessing technological initiatives to engage parents more in their children's learning, with Cathy also focused on celebrating the inclusion of features of the family culture. Families connected with schools in their own space, time, and way. The introduction of ICT and/or culture appeared to help break down the physical and logistical barriers experienced by parents, thereby overcoming the systemic lock-in referred to by Snyder (2013). This example shows the successful use of ICT and/or culture for connecting parents more successfully to the growth and development of their children at school. 
This section discusses the network interconnections and support mechanisms. Constraints to this principal leadership practice pattern were reduced by: the sharing of power, reduction of stressors, and the empowering of others often though simple conversation. Additionally, pattern momentum was increased by focusing on engagement in the learning process, supporting community safety and family wellbeing, and linking parents to the growth and development of their children at school often through ICT or the celebration of culture. This pattern connected learning excellence to aspects of sustainability.

\subsubsection{Theme 4 - Confronting and addressing injustices}

Theme 4 explores the LSE principal leadership networks' awareness of the effects of social positioning on learning. The theme links to the LSE specific 'teaching and learning inequalities' pattern outlined at the end of the literature review. In theory, this pattern allowed LSE learning communities to more successfully engage in teaching and learning (Bishop, 2011; Shields, 2013). This section discusses how the networks set about confronting and addressing injustices in practice and in particular how they: recognised the impact of poverty on learning and workload, challenged organisational school structures, managed student and whānau and teacher needs, took a political stance, addressed injustices, and ensured resources matched the needs of the school and its community.

Each principal developed their own thinking around the impact of poverty on learning. Amber was driven by her desire to do the right thing. Cathy had experienced what it felt like being on the outside of the norm and willingly interrupted cycles of segregation and poverty. Both Amber and Cathy questioned national assessment tools, varied test conditions, moderation techniques, and changes to leadership policies. Both principals were aware of the negative impact these changes were likely to have on student self-efficacy and the quality of teaching and learning. They also expressed concerns about the impact of policy changes on their students and the LSE communities they served, and in response, strategised decisions and actions that would have the most positive impact on the community. This finding is resonant of Aristotle's concept of practical wisdom or the 'mean of virtue' (Dunne, 1993). In this instance, the principal's personal leadership theories informed their practice according to their interpretation of contextual factors, and through the lens of their personally-held 
morals. This suggests that both principals were grounded in what they believed was right rather than merely attending to the expected.

The LSE context impacted on a principals' workload. At both Acacia and Cedar schools, parents and teachers acknowledged the enormity of the principal role which included that of manager, lead learner, teacher, social advocate and entrepreneur. Amber's network questioned her ability to keep up with growing demands and the impact this had already had on her health. Cathy's network (namely teachers) questioned their own ability to keep up with demands and Cathy herself admitted to this being unmanageable in the longer term. As indicated by Hallinger (2016), where the role begins and ends is vague. Concentrating on what matters is not as simple as being a good leader as suggested by Rice (2010), since problems are becoming more and more complex and therefore more and more interconnected. Funding, staffing, and accountably measures all put pressure on a principal's time (Wylie et al., 2018). In this study, the principal leader in an LSE school works exceptionally successfully to negate the impact of inequity for the LSE collective, making efforts not supplementary but part of business as usual - but whether this is sustainable is doubtful.

Specifically, principals worked hard to mould their role to meet the PLD needs of teachers. Amber took teachers' classes so the teachers themselves could partake in PLD. Her direct involvement in one-on-one teacher PLD was not possible due to funding reassessments, a reduced school roll, and fluctuating aiga/whānau contributions. Cathy, however, could work more directly and to a greater depth, to explore complex learning dilemmas during teacher observations. This involvement made it more likely that structural changes could occur. For example, by being involved in teacher observations it became clearer to Cathy that a greater focus was needed on key competencies and this saw a change in teacher planning. A larger staff and more stable funds made this possible. This study found that finances largely dictated principal involvement in PLD. The leadership BES (Robinson et al., 2009) states that school leaders who participate in teacher professional learning are investing in involvement that highly influences student outcomes, and Wylie et al. (2018) comment that strengthening professional inquiry and evaluating practices are important levers for change. Acacia, as the smaller of the two schools in this study had little choice in the matter. My study highlights financial barriers that make it a challenge for the principal leader of smaller schools to be directly involved in PLD to 
a depth that is likely to see systemic change. This finding exemplifies the work of Ladson-Billings (2006) and Shields (2013) who suggest there is systemic inequity evident when the resources required to achieve for cohorts of greater diversity are not made available.

The principal networks challenged school organisational structures that constrained engagement. Amber concentrated mainly on diversifying the principal and teacher role, so the wellbeing of students was inherently possible. Cathy worked towards a less subject-centred and fragmented programme of learning and often had multiple reasons for making a change. Neither principal was satisfied with the structures they traditionally worked within. This research indicates that a principal leader's willingness to 'unbundle' their schools (Hess \& Meeks, 2010) or create more flexible structures (Parrett \& Budge, 2012) is essential if unhelpful school structures are to be dismantled and replaced. Walker et al. (2011) see the creating of more flexible structures as a key leadership challenge: "connecting, disconnecting and reconnecting pathways" (p. 3) and Fullan (2009) believes restructuring schools is crucial if complex systems are going to transform and gain traction. Structures related to role and programming were redeveloped and new ways of doing things evolved to enhance engagement opportunities.

The networks managed student and whānau health needs. At Acacia, there was evidence of family members experiencing chronic stress and the network helped arrange GP visits as required or accessed the local SWIS. At Cedar, work with the cluster-wide health nurse regarding dental hygiene was ongoing, a one-off health research programme was encouraged, and healthy food provided. A NN dedicated to health and welfare services was also evident at Cedar School. Students and their aiga/whānau also required dental and mental health services. Such health and wellbeing factors are seen by Parrett and Budge (2012) as integral to learning as they can impact cognitive success. The principals in these two schools show that LSE principal networks are "spending time trying to compensate for social disadvantage by replicating the services middle-class children already get in their homes, at school" (Carpenter \& Osborne, 2014, p. 29). However, improvement of access to health services is ad hoc, and provided through goodwill. 
The networks addressed injustices by redistributing resources towards aiga/whānau to ensure greater participation and engagement. This showed an awareness of the hidden costs of schooling (Parrett \& Budge, 2012) which included providing lunches for children, having students attended school rather than looking after younger siblings, and paying for extramural expenses such as camp and swimming. Internal school funds made up from private donations were utilised for families who needed extra support with food, clothing, and extra-curricular costs. Efforts to provide for aiga/whānau financial shortfalls were not only helpful, but essential. Such social challenges demand that leaders adopt creative ways to counter social disadvantage and support the work of Anderson and White (2011) who suggest leaders harness new and existing opportunities for themselves and their communities. Baker (2018) argues that money alone is not the answer, but in these schools increased financial input was necessary for the improvement of educational outcomes.

Both principals addressed injustices by positioning themselves politically, often resisting the regulatory and accountability pressures that emphasised the achievement gap in narrow ways. Both were a part of the education union, with Amber actively involved in discussions regarding forthcoming changes to school leadership. Cathy was confident to challenge the disparity between assessment policies and actual practice by writing letters and meeting with MOE officials. Cathy especially spoke of neo-liberalism and the effect that colonialism has had on Māori and Pasifika peoples, showing her willingness to start from the position of injustice spoken of by Shields (2013). This finding is in opposition to Normore and Brooks (2017) who argue that most principals are only faintly aware of national and global politics and how politics influences their work in schools. Principals were aware of how national politics plays out in a school setting.

As such, the principal leadership networks worked to effect change by ensuring resources matched the learning needs of its learning community. At Cedar, it was particularly evident that PLD on offer was not always suitable. The science and ICT PLD, for example, was not timely and a gap was identified by the leadership team between advice and what the school already knew and needed. This finding identifies a mismatch between PLD needs and PLD availability and supports findings by the Professional Development Advisory Group (2014), which suggest that current PLD initiatives are not well matched to school/kura processes or learning needs. My 
research indicates that for leaders to ensure teachers become adaptive practitioners, experts coming into the LSE context must work as much on contextual considerations as they do on the content knowledge gaps. This in fact is a recent suggestion made by the MOE (2018). A more significant connection between what is learnt by teachers and how it is adapted to context will facilitate more timely and appropriate PLD.

Specifically, the networks identified additional ICT resourcing requirements. At Acacia, the main concern was securing reliable technology and its security whereas Cathy provided in-house ICT PLD and devices through a private trust and worked to involve parents in learning about the technology also. All funding for the development of ICT was, however, temporary. This finding identifies with empirical NZ research that identifies aspects of the digital divide (Lips, Eppel, McRae, Starkey, Sylvester, Parore \& Barlow; Pick \& Sarkar, 2015; Sarkar, 2015; Starkey, Sylvester, \& Johnstone, 2017). Both resources and knowledge were lacking. My research suggests that a lack of ICT resources and time dedicated to their use in the smaller of the two schools created a negative feedback loop that likely amplified the achievement gap. As is evident when contrasting Acacia and Cedar schools however, is that facilitating ICT engagement required working to integrate ICT into the teaching and learning, reducing the effect of financial constraints, and addressing teacher competency. Working these three aspects together was likely to reduce the achievement gap.

The networks also ensured school and neighbourhood facilities were always accessible. While both schools required extensive property maintenance, this did not stop Amber and Cathy offering the school facilities for holiday programmes, music lessons, and weekend whānau gatherings, either free of charge or at minimal cost. Amber worked exceptionally hard to campaign to keep her school and other local community facilities open and available to the public. My research found that the school facilities were used both in and out of school term-time to extend learning opportunities and social cohesion or alternatively what Parrett and Budge (2012) refer to as widening the school's mandate to better serve of local needs. Blackwell (2013) argues that investing in a well-maintained school is the bedrock of the local community, with Jacobson et al. (2007) adding that there is a growing need for principals to advocate not just for school development but for wider social community development in poorer neighbourhoods. Maximising engagement opportunities both in and out of school time was possible through the sharing of school facilities. 
The potential that prosperous schools in the wider community held for the network was recognised. At Acacia, Amber accessed sports equipment and an art tutor from private schools, and Cathy sourced interactive whiteboards from the intermediate school. ${ }^{44}$ My research findings show the principals used their connections to share resources and teaching expertise. Ainscow (2015) recommends a focus on such levers to encourage support and development of interdependence among groups of schools so that raising standards for all students in that district is the goal. Rashbrooke (2013) however goes farther, suggesting that it is the responsibility of those with wealth to create the conditions needed for equity. In NZ, Wylie (2012) makes a convincing case that stronger local and central connections are not just preferred, but essential to addressing the fundamental challenges faced by schools today. Niesche (2016) supports this theory, positing that social justice leadership is not just for those in disadvantaged schools but is the responsibility of all educators. By engaging prosperous schools in a shared aspirational vision for all students in their wider community, not only does responsibility for the education of LSE students become shared, but the boundaries of all schools involved become more active, further improving the likelihood of greater social cohesion. This study shows that connections extended to different schools in the area for improved resources.

This section discussed learning inequities. To reduce constraints to this principal leadership practice pattern and increase momentum, the network ensured that principals: were grounded in what they believed was right and were au fait with the national political picture; redeveloped structures related to role and programming; addressed teacher competency by exploring the links between new learning and context; worked to integrate ICT into teaching and learning, and shared school facilities. Equity was obstructed by the pace set by principals to negate the impact of poverty on learning, when resources required to achieve for cohorts of greater diversity were not made available, and when improved access to health services was ad hoc and financial input inadequate. This pattern connected learning excellence to the ability of the principal leadership network to develop a stronger sense of place.

\subsection{Exploring systemic capabilities}

44 Intermediate schools are schools specifically for Year 7 and 8 students (12-13-year-olds). These students are pre-secondary school. 
The principal leadership practice network sees the coming together of the four patterns explored above and contributes to the movement of the network whole. Very often, the momentum of leadership practice patterns was destabilised by contextual factors, moving networks further away from the possibility of transformational change. The capability of the network can be explained through the concepts of stability and flow, bringing into the foreground the nuanced differences between the principals' patterns of practice, and serves to confirm or negate the summaries at the end Chapter Five and Chapter Six.

Both stability and flow co-existed in the networks. Each of the networks had a clear vision for learning, action plans to implement the school vision, as well as policies and interventions. These all produced stability. However, at times practical steps were unclear. For example, both principals were uncertain how to proceed with the development of literacy in the senior school and/or ICT/science. Hargreaves and Fullan (1998) suggest that while you can set direction and insight, you can never know exactly how to advance. The networks reacted by exploring the problem, hypothesising possible solutions, trying new things, and reviewing the result. Often in a complex learning environment, "the only way forward is to focus on experimentation and learning" (Levin, 2006, p. 198). This study shows that leadership practice was about experimenting for change.

The smaller LSE context of Acacia School, was subject to greater instability. The small size of Amber's networks made it more difficult to stay on course since the energy and resources needed to balance the internal dynamics and effects of the environment were effectively less, and the context more volatile and consequently less able to be positively influenced. The smaller staff contributed to greater instability, even though Amber worked to broaden network membership. With a smaller network overall, Amber worked hard merely to avoid chaos and consequently was less likely to gain any significant forward traction. At times, the network was in danger of tipping into regression, saved only by the harnessing of resources, and maintenance of healthy relationships. My research shows that the volatility of the environment and an enormous amount of continuous change directly affect what principals spend their time on. This finding is in opposition to the work of Marzano et al. (2005), who suggest that school leaders are understating the enormity of change needed. I suggest that Amber was aware of what was needed but was instead caught up in managing stability in response to 
regressive exogenous changes, so the network did not fall into chaos. Principal leadership practice at Acacia worked hard to keep the network on course to maintain the teaching and learning already occurring. Maintenance and finding solutions were at the centre of this network.

The bigger network was more stable than the smaller network. The Cedar school network showed more evidence of developmental change and emergence, suggesting that more participants and incoming resources saw a greater capacity of the system to cope with change and to re-stabilise itself. The volatility of the context did not challenge Cedar and Cathy in the same way. Cathy's principal leadership network was a match for unstable environmental conditions (Goldstein et al. 2010, p. 1), meaning the behaviours of the collectively produced positive feedback loops allowed maintenance of desirable changes and allowed for the possibility of emergence. Greater stability meant that at times Cathy could work through the complexity and create movement and change. Cathy harnessed resources, pedagogical improvement, and strong relationships to achieve this. Principal leadership practice at Cedar worked to move the network forward and improve the teaching and learning. A learning disposition was at the centre of this network.

Both principals noticed when the network was restricted. This included when there was a lack of student voice, when there were student behaviour and attendance issues, contrary viewpoints on the levels of success attainable, and wider education policy accountability restrictions. Weick et al. (2005) draw attention to a sensitivity to detecting what is not right and improving it, which these principals regularly did. The networks were successfully nudged by the principals towards preferred patterns of practice, using humour, seeking out student voice, implementing a behaviour modification programme, talking to students and parents directly, chatting with local councillors, and writing letters to the MOE. Principal leadership practice worked to nudge the system forward or, at the very least, keep momentum going, by having students present and engaged in an ongoing cycle of learning. Indeed, both principals worked well with ongoing tensions.

There were individual differences between principals that may have contributed to the varying degrees of stability of the networks. The main considerations are those related to a principal's availability to lead learning, reject deficit thinking and successfully 
engender a spirit of reciprocity. First, principals worked to expand notions of success and focused on engagement rather than achievement. However, Cathy was significantly more engaged in the teaching and learning process than Amber. It was acknowledged by Amber however, that a more adequate staffing allowance would have given her more time to go into classes and observe teachers. It appears that the principal in a smaller LSE school must work harder than the principal in a larger school to sustain their learning network in the face of inadequate support from the state. This finding is evidence of education debt (Ladson- Billings, 2006) and appears to be an issue of equity rather than will and/or skill.

Second, both principals had to counteract less than positive views of school community/community members. Amber's "mindful ignoring” of network members" deficit thinking related to aiga/whānau, stood in contrast to Cathy's more ongoing and direct confrontation of the issue. Cathy's approach seemed more effective in challenging internal deficit thinking. Amber however, was noted to understand the high stress her teachers were under and it appears that Amber has used an alternative strategy for maintaining her patterns of practice, while also working to address deficit mentality. Addressing deficit comments 1-1 and in a less direct manner, Amber appeared to be able to show empathy for her staff. Goleman and Senge (2014) refer to this as EI. An ability 'to recognise own and others' emotions and use this to guide thinking and behaviour" (p.248). This means deficit thinking is instead examined over time with consideration for the stresses of the context. Whether this is more or less effective than a direct, more rapid approach remains unclear.

Third, feedback loops seemed to indicate that the principals did not have similar success engendering a spirit of reciprocity. Amber's nested network members appeared to be more inclined to leave a significant portion of the actual work in the learning community to her alone, especially with regard to aiga/whānau engagement. The Acacia staff questioned Amber's ability to keep up with the growing demands. However, at Cedar, Cathy's network, namely teachers, questioned their own ability to keep up with demands and the pace Cathy set to address needs. While Cathy appeared to actively engage members more effectively than Amber, Cathy herself admitted to this being unmanageable in the longer term. This supports the request by Wylie (2017), that the principal leadership workload in NZ be urgently revised because of significant numbers of principals experiencing high or extremely high levels of stress. It appears 
that no matter how effective principals are at developing reciprocity between network members, the principal role in its current form, is unsustainable within the LSE context.

Considering the stability and flow of the network overall, as well as the varied capabilities of the individual principal leaders, the network worked hard to keep itself on the preferred trajectory and influences principal LSE practice. The impact of external forces often reduced this effectiveness. While encouraging commitment from their network members to 'do the right thing' or 'break the cycle', the LSE principal leaders were experiencing such relentless disruption to their feedback loops from outside and within the LSE schools, that at best the leadership network could progress with only some possibility of emergence and thus transformation, especially in Amber's case. This finding supports the work of Reed and Swaminathan (2014) who concede that LSE settings narrow and constrain the effect of policy on practice, despite the important work done by leaders to mitigate the contextual influences on learning. The study appears to show that without the wider system intervening, school learning communities are becoming increasingly fragile and may even lose energy and perish (Bendikson et al. 2015).

However, through the identification of the four patterns of practice and the subsequent tipping points, a lifeline becomes evident. While tipping points identify systemic weaknesses, these same tipping points according to Capra (2016) also offer the greatest possibility of progress and change. By putting further energy into the areas of community-school partnerships, resourcing and PLD, education may be better able to not just adapt to the needs of individual LSE communities, but provide emergent change that develops and even transforms education as a whole.

\subsection{Chapter Summary}

This chapter has explored leadership in practice for educational success. Success in this study includes: a consideration of the whole person so the cultivation of a diverse range of talents, successes and identities is possible; a focus on learning as a process of reflection and improvement that personalises formative measures of success 
alongside collective, summative measures; and a concern with eco-social wellbeing so that present and future successes are mindfully considered.

The study showed how the entire living network was centred around various patterns of practice that were found in a less complete form in the literature. The patterns became evident when people and the LSE contexts they inhabit, were described in a complex manner and included: building the network, integrating multiple perspectives, facilitating cohesion and confronting and addressing injustices. The patterns were however very regularly destabilised by the more extensive networks within which they worked. Disruptions came from the community and wider education systems especially.

The research explored how practice patterns occurred in motion. Three main vulnerabilities destabilised the communities of learning: resourcing vulnerabilities included inadequate BOT expertise, insufficient and reducing funds and inconsistent funding solutions and resources that did not keep up with a growing diversity of cultural and learning needs within the school; PLD vulnerabilities included growth opportunities that did not meet teaching and learning needs, the speed at which solutions to more complex learning problems were found, limited teacher knowledge in areas such as ICT, assessment and science and, the perception of inaccurate and unreliable test results; School - community relationship vulnerabilities included unreliable learning relationships between home and school, differing confidence between parent and school expectations of success, tensions between whānau/aiga in the wider school community, high student absenteeism and/or inappropriate social behaviours, a mismatch between public and private views regarding the quality of education available and increased competition between schools.

The stability and flow of the network coexisted and both principal leaders were highly skilled at building broader networks of people dedicated to noticing restrictions, and making changes that nudged the system forward. To address vulnerabilities principal leaders practised more from the position of practical wisdom or next practice to keep patterns of success moving. Leveraging in the current principal leadership networks allowed for: 
- a focus on values related to diversity and belonging and social justice and equity that led to social change and the rebalancing of power differentials.

- emphasis on drawing the network together to ensure collective learning and wellbeing

- a focus on learning rather than impediments to learning such as school absences, ill health or lack of aiga/whānau input

- a focus on solutions that were future focused and systems orientated

- a focus on the wellbeing of students and aiga/whānau

- accessing what functioned in the system and pushing the boundaries of the system further without losing sight of the bigger picture.

Regardless, the capability of the network to evolve meaningful change is questionable. The volume of problems addressed was complex and time-consuming and the constant volatility of the context, maintains the tail of underachievement which works to undo progress made. The wider systems within which principal leadership is practised need to make changes if peak levels (Adams, 2015) are to increase and principal leadership is to generate transformation in schools. 


\section{CHAPTER 8 \\ Looking back on the journey}

'Ka koroki te manu - creating our tomorrow'

\subsection{Chapter overview}

In this final chapter, the research is reviewed starting with the initial research objectives. Findings are drawn together to answer the research question/s. The second part of the chapter projects forward, reflecting on the contributions and implications. It concludes with recommendations, study limitations, and future research considerations. The recommendations contribute to supporting a more systemic approach to reducing the NZ student achievement gap, which up until now, has appeared unsolvable.

\subsection{Objectives and questions}

The thesis aimed to investigate how principals practise leadership in the LSE school context to promote success. More specifically, the research objectives were to:

1. recognise principal leadership practice as a complex system so systemic solutions might be identified to underachievement

2. understand the ways principals think and interact within their context to encourage home-grown solutions and locally authentic perspectives of educational success

3. identify the likelihood of transformational change when viewing principal practice from within a complex, contextually driven, system of schooling.

The literature review identified that a clearer picture was needed of the strengths of a school principal when working with evolving contextual dynamics to harness progress and transformation in an LSE context for localised notions of success. 


\subsection{The research question/s}

The complexity of how principal leadership practice emerged in an LSE context for localised notions of success has not been previously considered in the literature. Four sub-questions explored different aspects of the gaps in the literature:

1. What leadership practice patterns are in evidence in the daily life of a principal?

2. How is LSE principal leadership influencing of, or influenced by, context?

3. What beliefs, values and ideas underpin a principal's practice patterns in the LSE context for educational success?

4. What do the stakeholders in the LSE environment perceive as educational success?

The central question of the research became:

'How do principals practise leadership in an LSE primary schooling context for educational success?'

Chapters 3 and 4 outlined the theoretical framework and methodology. Three connected theories acted as the foundation on which to build a methodology fit for purpose. First, Bronfenbrenner's (1979) ecological systems theory was used as a springboard for focusing on people and context. Second, complexity theory acted as a framing for focusing on the interrelationships occurring between the principal and their leadership network components. Finally, included was Capra's systems view of life (Capra, 1997). This latter theory highlighted the natural movement and change that occurred in process, exemplifying principal leadership as a living system in a constant state of becoming. Combined in this additive way, the three theories constituted a sophisticated holistic frame of thinking for describing and exploring leadership as an eco-social process making the all-important link between theory and practice. The design of the investigation was founded on the constructivist and transformational paradigms and the investigation proceeded using an emergent, embedded case study. 


\subsection{Answering individual research questions}

Calling on the data description the research sub-questions are answered.

\subsubsection{What leadership practice patterns are in evidence in the principal leadership network?}

Patterns are the essence of life and offer a more holistic and expanded view of practice. Such patterns helped create non-hierarchal, interdependent and purposeful connections.

Evident after some months of familiarity, analysis and reflection on the data were four patterns that brought the daily practices and case study levels of scale together. First was building relationships. This pattern aimed to soften the network boundaries and ensure an inclusive learning community. The second was integrating multiple perspectives. This pattern aimed to work to ensure the inclusion and wellbeing of all network members. The third was creating reciprocity. This pattern worked to meld strong personal identities that contributed to an even stronger collective and sustainable culture. The fourth was confronting and addressing injustices. This pattern was grounded in strongly-held beliefs which permeated micro-political day-to-day actions and ensured a future-focus. The patterns result from a complex interaction between people, context, and interpersonal learning in the principal networks and worked primarily to create high engagement levels, especially of students.

\subsubsection{What beliefs and ideas underpin a principal's engagement in their context?}

The beliefs and ideas that were seen to underpin principals' leadership engagement, aligned with the perceptions of the wider network and included:

- beliefs around diversity and belonging and the ideas associated with empowerment and building strong, respectful relationships

- beliefs that highlight equity and social justice and the idea that difference is a welcome strength

- a belief in being solutions and future-focused, with the idea that solutions must consider the wellbeing of people and place, now and in the future

- a systemic view of the world and the notion that solutions are somewhere in the system. 
The beliefs and ideas inspired deeper schooling ecological outcomes. Education is, therefore, not just about getting a job and making money, but wellbeing and projecting a world where a quality of life is possible for future generations.

\subsubsection{In what ways are principal leadership practice patterns responsive to and influenced by their context?}

The answer to this question explains how the principals, and others in their network, think and interact within the context and how they are restrained by, or use, context as systemic leverage.

\section{Influenced by context}

Principal leadership practice patterns were made vulnerable by contextual influences related to resourcing, and PLD and school - community relationships. These vulnerabilities constrained the flow of engagement by affecting:

- the ability of the principals' networks to stay focused on relationships that matter, develop shared goals, and improve engagement at the network periphery

- the quality of work produced by the principals' networks and the ability to both fulfil current learning needs and project forward to future learning needs

- the ability of the principals' networks to address problems accurately. This includes developing the thinking required to solve puzzles of practice, make a change, and focus on learning rather than accountability measures.

The principal leadership networks in this study worked to address these constraints as issues of injustice.

\section{Influence on context}

Principal leadership practice patterns reduced obstacles to learning caused by poverty and were led by the principal leader. Three main practice patterns self-regulated and even offered leverage in the leadership network.

First, the principal leadership networks developed a collaborative culture. The principal mainly collaborated with staff and support staff, governance, aiga/whānau and health-care professionals. Some evidence of collaborating with students was also 
evident. The reasons for collaboration included: to release teachers; share resources and programmes; encourage a shared approach to problem-solving; share an understanding of what learning looks like; offer pastoral and cultural support; develop flexible authentic, compassionate relationships; and improve and secure the school grounds.

Second, the principal leadership networks developed a culture of change. The principal leader challenged staff, BOT, aiga/whānau, government bureaucrats and politicians. Change processes evident included: pushing forward with less popular initiatives; working to change the narrative around school attendance; working to evolve the investment of aiga/whānau in school life; diversifying network membership responsibilities and roles; calling out narratives around deficit theorising; and challenging policies unsupportive of success.

Third, the principal leadership networks were adaptive. By using a type of next practice and practical wisdom, the principal leader worked with uncertainty by:

- ensuring sensitivity to the functioning of the whole

- effectively using relationships

- improving welfare accessibility

- managing budgetary constraints or finding alternative funding sources

- enhancing the knowledge and reputation of the school

- celebrating the school's point of difference

- guaranteeing a fair distribution of available resources

- encouraging families to change their child's success narrative

- accepting teaching and learning as complex work

- accessing mentors

- developing a widening curriculum

- using accurate and creative ways to show progress.

As a result, both principal leaders in this research were proven to be highly skilled, adaptable and committed to building networks of people dedicated to improvement and change. 
The self-regulating principal leadership network constrained the flow of problems by adapting to produce both progressive and developmental movement. The combination of the progressive (most common) and developmental feedback loops makes the principal leadership practice network self-organising and able to work towards a shared vision and values.

\subsubsection{What do the stakeholders in the LSE context perceive as educational success?}

For students to flourish, rather than merely just survive, educational success is perceived as inclusive of the whole child, especially aspects of success that can be related to cultural identity. Success, while accepted as summative, is also a journey focused on continually engaging to add value, while working to also recognise it in its many concrete and abstract forms. Finally, educational success has a deeply ecological belief system and is considerate of present and future social and environmental responsibilities.

\subsection{Answering the question overall}

The main purpose of systems theory is to explain how nonlinear, complex systems act. Specifically, it explains the behaviours and governs the characteristics of living systems. Calling on the description and exploration, the overall research question can be answered in a holistic frame of thinking. The characteristics of principal leadership practice involves principals demonstrating:

- a strong personal foundation on which to construct their narrative but also with an embedded place in the community (centrally visible, accessible and mobile across the network) where they prioritised relationships, and connecting their learning community

- $\quad$ varied personal skills and social networking skills (including a sense of humour) that helped maintain social interactions

- acceptance of teaching and learning as complex and at times an unknown quantity while also able to engage reflectively in the teaching and learning process with hope 
- a strong moral compass to frequently identify, monitor and disrupt opinions related to difference, embrace a diversity of perspectives and empower those traditionally silenced, especially students, aiga/whānau, and those on the fringes of the school community

- a specific awareness of politics, at both a micro-level and macro level and the policy-practice dynamics that occurred between leadership, learning and governance.

The characteristics of principal leadership practice overall involve principal networks that are:

- diverse and cohesively connected, aligned through an emerging shared vision and identity focused on student achievement and student and aiga/whānau health and wellbeing

- made up of a robust and stable core set of relationships, including relationships which spread beyond the school's physical environment and into diverse community members practising varied disciplines for more significant social capital

- evolving a dual focus on academic and social wellbeing

- developing an inclusive, holistic, yet critical curriculum that cultivates a diverse range of talents with special consideration of development in technology and science

- focused on engagement as a learning process immersive of agency, self-efficacy and behavioural development so that individuals develop their form of futurefocused expertise that is challenging of the diversity of engagement ideas and seeks a tighter coupling between civic responsibilities, academic learning, and wellbeing

- concerned with a present and future considerate school infrastructure that is flexible and adjusted to ensure safety, the availability of technology and other teaching and learning resources, funding opportunities, teaching and leadership relationships, PLD programmes, and the school's public image. 
The desirable characteristics of both the principal leader and their network requires personal and collective skills and beliefs that align for an inclusive, holistic and engaged community of learners both now and into the future.

\subsection{Contributions}

Relatable to the research objectives: identifying systemic solutions; understanding the influence of context; and deciding on the systems capability for transformation, are the three main contributions of this study.

First, in viewing principal leadership from a systems perspective four main patterns of practice showed leadership as an eco-social process: building the network; integrating multiple perspectives; facilitating cohesion; confronting and addressing injustices. Within these, three points of vulnerability, which are also important leverage points were identified. These were professional learning and development, communityschool connections and resourcing. These specific areas offer LSE principal leaders, school leadership teams, as well as the Ministry of Education and policymakers suggestions for where to focus time and energy, for a greater likelihood of disrupting the current trajectory. While this study did not set out to explore issues of inequality, it certainly suggests that the current trajectory of success indicates systemic resourcing inequities.

Second, this study has demonstrated the centrality of context for a more complex understanding of the LSE leadership practice rhetoric. The methodology purposefully moved away from reductionism, by creating a theoretical framework grounded in the works of the researcher's personal philosophy, Bronfenbrenner's (1979) ecological systems theory, complexity theory and Capra's (1997) conceptual theoretical framing known as the system's view of life. The methodology repositioned context as an inseparable part of the data-gathering process. The theoretical framework kept the integrity of the nonlinear process and made it possible to present the network of principal practice for LSE student success as a living system of its own making, making the all-important link between theory and practice. At the same time, the research highlights the importance of a principal leader who can match theory to practice in context and in motion. 
Third, the likelihood of transformational change from within the current system is decided. The capability of the principal leadership practice network is described through concepts of stability and flow and shows, that no matter how competent the principal leader and the network at matching theory to practice, the contribution grapples to be transformational. Even with nudging and encouraging, the constant volatility of the context maintains the tail of underachievement which works to undo progress made. Thus, the wider ecological system within which principal leadership is practised need to make changes if peak levels (Adams, 2015) are to increase and principal leadership is to generate transformational change.

\subsection{Implications}

Reducing the achievement gap requires a cohesive, positively interdependent, developing portfolio of initiatives and policies that lie within and beyond the school setting. The school principal leadership networks, while highly capable of contributing to reducing the achievement gap, are still only a partial solution. Broader aspects of poverty must be addressed alongside improved teacher quality and leadership and a change in the system-whole to bolster greater achievement possibilities. Recognising principal leadership as a complex adaptive system, means recognising the need for multiple changes at once.

Understanding the interplay between practice and context requires more sophisticated thinking and an ease with the unknown. There appears to be a need to develop the ability of leaders to navigate complexity as a 'new normal'. Leaders need to build a new type of knowledge about the features of complex systems and how they behave. Indeed, in times of rapid change and an unimaginable future, the only way to move forward is to critically think about the impact of actions, and adjust when the evidence shows unintended and undesirable consequences. Additionally, solutions discovered using a complex systems approach may uncover new or unrelated change suggestions that not only highlight answers within the system proper but also beyond the current membership and its boundary. Systems thinking will help evolve both individual and collective behaviours concurrently.

For transformational change to be more likely, bravery is required of individual leaders. Principal leaders have the potential to be a political force for good and are 
well-positioned to be critical change agents. There is a need for principal leaders to speak plainly about what they perceive are inequities in the system, no matter how uncomfortable that may be. The tail of under-achievement must be treated as a crisis, and highlights a need to focus on equity. For example, smaller LSE schools have immense difficulties just because of the number of changes regularly occurring and fewer people and resources to adjust to that change. Support in LSE schools needs to be far more intense if the achievement gap is to narrow. Staying on top of continually emerging issues is problematic. Where inequities are addressed, more time and resources should be available to principal leaders and their schools'. Additionally, definitions of achievement need to be broadened so that alternative ideas about knowledge and skills can be valued and supported.

\subsection{Recommendations}

The need to broaden perceptions of both the problem and possible solution/s was identified in the introduction. The holistic systems approach used in this research has allowed principal leadership to be seen to have three susceptible points of inflection for systemic change. These are community-school partnerships, PLD, and resourcing. These areas of vulnerability, with greater resourcing, are likely to create stronger links between practice and theory. It is at these three tipping points that practice, professional development and policy recommendations are made.

\subsubsection{Practice}

Principal leaders need to be able to work independent of others and interdependently with others. The findings from the research strongly indicate a need for leadership practice to be:

- focused on developing a diversity of ordered and disordered learning-focused relationships

- focused on relationships at all network levels. The periphery of the network should include a focus on university connections as well as connections with policy and political levels of government to help broaden the principal's sphere of influence. Partnerships with private businesses also have the potential to increase affordance networking so that resources for all children 
are made possible. Relationships at the centre of the network require a focus on students and aiga/whānau to ensure learning engagement is maximised.

- focused on relationship quality. Relationships need to reflect progression from a mere service-agent partnership to a partnership that is reciprocal, proactive and multi-faceted.

Altogether, it is the breadth and depth of relationships that will allow principal leadership practice to cope with natural change and external stressors, while remaining focused on school vision.

\subsubsection{Professional Learning and Development}

The research findings suggest the need for PLD that brings principals alongside middle leaders. PLD themes might include: collaboration; the development of personal theory, practical wisdom and next practice; CAS and leadership change theory exploration. These will support a greater understanding of the complexity of a principal's network in practice and help leaders to bridge the gap between their own and others' ideals.

The research found that principal leadership networks noticed, changed or dismantled structures and processes that held back progress. PLD that focuses on timely support of leaders to create new processes and structures may therefore also be useful. However there also needs to be a way for any progress to feed into policy.

\subsubsection{Leadership policy}

Various policy imperatives are recommended that may help to future-proof the educational leadership practice patterns of: building the network; integrating multiple perspectives; facilitating cohesion; and addressing and confronting injustices.

To build strong networks, findings from the research strongly support policy that is centred around better supporting LSE families and their communities. Firstly, there is a need to develop policy that creates a more interdisciplinary approach to improving home-school relations. Policy outside of education that supports the involvement of parents in school life might for example include employee rights, access to mental health services or budgeting services. Secondly, wider policy initiatives that affect LSE communities require input from principals. Policy work needs to be more evidently driven in NZ by the involvement of local level actors, so reform is 
continuously refined and improved by all involved. Developments around housing policy and the living wage are two policy examples requiring principal input. The research shows an already close link between education and health, and recommends this as a good starting point.

Two considerations identified in the research would support the integration of multiple perspectives. First, is the need for policy to be centred around developing broader notions of success. This includes giving weight to local ideals that are inclusive of personal and cultural identity. Second, is the need for policy that directs supplementary resources to LSE schools so that a holistic curriculum is more accessible to all students, no matter the state of their aiga/whānau resources.

Research findings strongly indicate the need for policies that dismantle rigid accountability measures and unhelpful school structures for greater cohesion. Additionally, it is recommended that future policies are designed in such a way, that draws leadership, learning, and governance practices closer together. Moreover, a MOE policy that details principal recruitment and retention responsibilities would help to address the issue of job satisfaction and time in role. This may help to address the unsustainable effort required of principals to negate the impact of poverty on learning, keeping them in their roles longer and thus reducing disorder.

Confronting and addressing injustices requires policy that addresses vulnerabilities referred to by Ladson-Billings (2006) and Shields (2013) as systemic inequities. The findings from this research specifically indicate that governmental agencies develop a policy that consider the effects of poverty on learning to guide all future education related decisions and to ensure this supports more inclusive practice across the education sector. Additionally, there is a need to develop policies that address low decile school shortfalls in opportunities. LSE school staffing, PLD and PR policies with funding attached are four examples for consideration. Providing a new staffing policy equation that allows LSE principal leaders to employee the staff required to meet the diverse community composition, including specialist ELL teachers, and is stable amidst the ebb and flow of the school role, is another. These policies would work to ensure that the resources required are made available to help connect learning excellence to social innovation, school culture and place. 


\subsection{Limitations}

Using a complex systems approach meant accepting that there are as many perspectives not included in the research. This case study focused in depth on one of the many components that make up the principal leadership network - the principal and how the network is configured with her at its centre. Consideration of the principal leadership network with an alternative focus is likely to look different and uncover different possibilities.

The data collected were not thoughtfully conceived from the outset because the theory emerged throughout the research process rather than from the beginning. The most useful data came from observations, yet only two of the observations looked in depth at natural communication patterns occurring. These were focused on leadership teams and BOT and provided a greater depth of evidence in these areas. This research identified networks of strong support such as education support workers, or networks requiring more connectedness such as aiga/whānau. A focus on these groups might help address the imbalance of data here and, additionally, uncover a different set of solutions and ideas.

This research was a case study and, as such, findings cannot be generalised. Overall this work therefore, supports the claim by Hess (2008) and Shields (2012) that it cannot be assumed that with different leaders, teachers, students and locations the same findings will result and the same patterns and tipping points will be beneficial. However, it may well also be, as claimed by Capra (2016), that living systems all work in the same way. He claims that as any living systems continually experience structural change, web-like patterns are preserved. Capra's alternative suggestion implies therefore, that the patterns identified in this study are likely to be the same for any principal leadership practice network. What would be different are the structures and processes. The alignment between four of the five leadership practice patterns in the literature review, and those found in practice, support this hypothesis. This suggests that the patterns identified are likely the same for any principal leadership practice network; what would be different are the structures, and therefore processes. This postulation requires further consideration. 


\subsection{Future research}

The study identified the need for a greater focus on how middle managers and aiga/whānau interact within the principal leadership networks to ensure these components of the system are used to their maximum effect and do not draw resources away from other functioning network areas. Both would weaken the influence of the leadership practice overall. Further studies could be done that focus on such components or even the interrelationships between them.

The study focused on patterns rather than specific components that contributed to patterns of principal leadership practice. It would be of interest to investigate specifically the place of personal attributes that are currently in the literature but not emphasised in findings, for example, EI and how this attribute helps the network maintain itself. Indeed, more work specifically on the place of humour in leadership in the LSE primary school setting in NZ would also be helpful.

The study did not cover a sustained timeframe, at most, the data were collected over a year. A lengthier time span would offer greater opportunity to identify emergence. A longitudinal study that focused on tipping point patterns that emerge over time would be useful to determine what positive natural changes occur most frequently and how these could be purposefully designed. Such a study would also help to confirm whether indeed the wider education system can support much-needed transformation or whether the system is merely maintaining progress in support of the status quo.

The study preceded some post 2017 changes to the NZ education system. It would be of interest for future researchers to repeat this type of research sometime after the recommendations of the taskforce are implemented to see if the changes have distributed the system enough to warrant greater possibilities for transformation.

\subsection{Chapter summary}

There is nothing novel in suggesting leadership is a complex phenomenon; however, a main contribution of the study is the use of a suitable framework to investigate and represent this complexity. This research improves the understanding of how principal leaders practise leadership in LSE primary school contexts for educational success, and proposes the need to highlight the teaching and learning context within the wider 
system, where aspects of restraint or leverage are recognised to influence the likelihood of quality practice and student success. Through this more sophisticated conceptual understanding, the principal leader and their networks were found to be powerful tools for confronting both input and outcome inequities. This research brings context into focus, not as an excuse for educational underachievement, but to broaden perceptions of success and to highlight potential, and perhaps even previously unrealised solutions, so that acting for the crisis that exists is possible. In doing so, it broadens the impact of educational leadership - a moving, changing, living system of its own making - with the possibility of transformation to 'do the right thing' for both people and planet. Indeed, a principal leader is akin to an ecosystem engineer, a leader who, from within their network, seeks to create other leaders, and alongside these others, collaboratively modify, maintain or transform the environment with the aim of developing the collective health and wellbeing of the place and living beings that inhabit the space. This reconceptualisation has implications for policy, practice and PLD offering a greater likelihood of collective success, health and wellbeing for all. 


\section{References}

Abram, D. (1996). The spell of the sensuous: Perception and language in a morethan-human world. New York: Vintage Books.

Ackerman, R., Mette, I., \& Biddle, C. (2018). The adaptive challenges of leadership in Maine schools. Maine Policy Review, 27(1), 9.

Adams, J. (2015). Principal leadership practices in high poverty K-5 model schools in Oregon. (PhD). Portland State University, Portland, United States of America.

Agirdag, O., Houtte, M., \& Avermaet, P. (2012). Why does the ethnic and socioeconomic composition of schools' influence math achievement? The role of sense of futility and futility culture. European Sociological Review, 28(3), 366-378.

Agosto, V., \& Roland, E. (2018). Intersectionality and Educational Leadership: A Critical Review. Review of Research in Education, 42(1), 255-285.

Ainscow, M. (2015). Struggles for equity in education: The selected works of Mel Ainscow. London: Routledge.

Airini, Anae, M., Mila-Schaaf, K., Coxon, E., Mara, D., Sanga, K., ... Ministry of Education (Eds.). (2010). Teu le va: Relationships across research and policy in Pasifika education: A collective approach to knowledge generation \& policy development for action towards Pasifika education success. Auckland, NZ: Auckland Uniservices, University of Auckland.

Alhadeff-Jones, M. (2008). Three generations of complexity theories: Nuances and ambiguities. Educational Philosophy and Theory, 40(1), 66-82. doi.org/10.1111/j.1469-5812.2007.00411.x.

Anderson, G. (2009). Advocacy leadership: Toward a post-reform agenda in education. New York: Routledge.

Anderson, J., Boyle, C., \& Deppeler, J. (2014). The ecology of inclusive education. In H. Zhang, P. W. K. Chan, \& C. Boyle (Eds.), Equality in education: Fairness and inclusion (pp. 23-34). Rotterdam: Sense.

Anderson, M., \& White, S. (2011). Resourcing change in small schools. Australian Journal of Education, 55(1), 50-61.

Asri, M., \& Tahir, L. (2014). The influence of principals' self-personality values towards their work culture. International Journal of Evaluation and Research in Education, 3(4), 228-235. 
Baker, B. (2018). Educational inequity and school finance: Why money matters for America's students. United States: Harvard Educational Publishing Group.

Baldwin, T. (2015). Culturally responsive pedagogy: A transformative tool for CCCU educators in multicultural classrooms. Christian Education Journal, 12(1), 97-117.

Barrera-Osorio, F., Fasih, T., Patrinos, H., \& Santibanez, L. (2009). Decentralized decision-making in schools: The theory and evidence on school-based management. Washington DC: World Bank.

Bateson, N. (2016). Small arcs of larger circles: Framing through other patterns. Place of publication not identified: Triarchy Press.

Bendikson, L., Durie, M., Gilbert, J., Robertson, J., \& Wenmoth, D. (2015). Learning for communities of learning. Wellington: Education Council, New Zealand.

Berger, J. (2004). Dancing on the threshold of meaning: Recognizing and understanding the growing edge. Journal of Transformative Education, 2(4), 336-351.

Berk, L. (2000). Child development. Boston: Allyn \& Bacon, Incorporated.

Berryman, M., SooHoo, S., Nevin, A., Arani Barrett, T., Ford, T., Nodelman, D. J., ... Wilson, A. (2013). Culturally responsive methodologies at work in education settings. International Journal for Researcher Development, 4(2), $102-116$.

Berson, Y., \& Oreg, S. (2016). The role of school principals in shaping children's values. Psychological Science, 27(12), 1539-1549.

Bezzina, M. (2013). Ethical blind spots in leading for learning: An Australian study. Journal of Educational Administration, 51(5), 638-654.

Bishop, R. (2008). Effective leadership for educational reform: Report to Nga Pae o te Maramatanga. Waikato: The University of Waikato.

Bishop, R., Ladwig, J., \& Berryman, M. (2014). The centrality of relationships for pedagogy: The Whanaungatanga thesis. American Educational Research Journal, 51(1), 184-214.

Blaikie, P. (2012). Should some political ecology be useful? The inaugural lecture for the Cultural and Political Ecology Specialty Group, Annual Meeting of the Association of American Geographers, April 2010. Geoforum, 43(2), 231-239. 
Blanchard, K., \& Broadwell, R. (2018). Servant leadership in action. United States. Berret-Koehler Publishers.

Blase J. (2005) The Micropolitics of Educational Change. In: Hargreaves A. (eds) Extending Educational Change. Springer, Dordrecht.

Blumen S. (2016) High achieving deprived young people facing the challenges of the 21st century. In: Ambrose D., Sternberg R.J. (eds) Giftedness and Talent in the 21st Century. Advances in Creativity and Giftedness, vol 10. SensePublishers, Rotterdam.

Bogotch, I., \& Shields, C. (2014). International handbook of educational leadership and social (in)justice: Volume 1 Retrieved from http://proxy.library.carleton.ca/login?url=http://dx.doi.org/10.1007/978-94007-6555-9.

Bolstad, R., Gilbert, J., McDowall, S., Bull, A., Boyd, S., \& Hipkins, R. (2012). Supporting future-oriented learning \& teaching: A New Zealand perspective. Wellington, NZ: Ministry of Education.

Bolstad, R. (2017). Digital technologies for learning: Findings from the NZCER national survey of primary and intermediate schools 2016. Wellington: New Zealand Council for Educational Research Press.

Bolton, S. (2017). Education equity in New Zealand: Success, challenges and opportunities. Wellington: Fullbright New Zealand.

Botton, A. D. (2008). The architecture of happiness (Reprint ed.). New York, NY: Vintage.

Boulton, J., Allen, P., \& Bowman, C. (2015). Embracing complexity: Strategic perspectives for an age of turbulence. Oxford UK: Oxford University Press.

Boyatzis, R. (1998). Transforming qualitative information: Thematic analysis and code development. London: Sage Publications Limited.

Brackins, L. (2012). Examining principals espoused beliefs and actions. Auburn University $(\mathrm{PhD})$. Alabama, United States.

Branson, C., Gross. S. (2014). Handbook of Ethical Educational Leadership. Abingdon: Routledge.

Briggs, A., Coleman, M., \& Morrison, M. (2012). Research methods in educational leadership and management ( $3^{\text {rd }}$ ed.). London: Sage Publications Limited.

Bronfenbrenner, U. (1979). The ecology of human development: Experiments by nature and design. Cambridge MA: Harvard University Press. 
Bronfenbrenner, U. (2009). The ecology of human development. Cambridge MA: Harvard University Press.

Brown, J. (2005). The world café: Shaping our futures through conversations that matter ( $1^{\text {st }}$ ed.). San Francisco: Berrett-Koehler.

Bryk, A., Gomez, L., Grunow, A., \& LeMahieu, P. (2015). Learning to improve: How America's schools can get better at getting better. Cambridge, MA: Harvard Education Press.

Bryk, A., Sebring, P., Allensworth, E., Luppescu, S., \& Easton, J. (2010). Organizing schools for improvement: Lessons from Chicago. Chicago: University of Chicago Press.

Budge, K. M (2010). Why shouldn't rural kids have it all? Place- conscious leadership in an era of extralocal reform policy. Education Policy Analysis Archives, 18 (1), 2-23.

Bush, T. (2010). Theories of educational leadership and management $\left(4^{\text {th }} \mathrm{ed}\right.$.). London: Sage Publications Limited.

Bush, T. (2008). Leadership and management development in education. London: Sage Publications Limited.

Byrne, D. S. (1998). Complexity theory and the social sciences: An introduction. London: Routledge.

Byrne, D., \& Callaghan, G. (2013). Complexity theory and the social sciences: The state of the art. New York: Routledge.

Cala, V., \& Soriano, E. (2014). Health education from an ecological perspective: Adaptation of the Bronfenbrenner model from an experience with adolescents. Procedia: Social and Behavioral Sciences, 132, 49-57. doi.org/10.1016/j.sbspro.2014.04.277.

Caldwell, R. (2005). Agency and change: Rethinking change agency in organizations. London: Routledge.

Callaghan, C. (2013). The Role of Professional Leaders in Creating and Maintaining School Cultures in Times of Change. Principal Sabbatical Report. Christchurch, NZ.

Campbell, J., \& van Nieuwerburgh, C. (2017). The leader's guide to coaching in schools: Creating conditions for effective learning. California: Corwin Press. Campbell-Evans, G. (1993). A Values Perspective on School-based Management: School Based Management and School Effectiveness. London: Routledge. 
Capper, C., \& Young, M. (2014). Ironies and limitations of educational leadership for social justice: A call to social justice educators. Theory into Practice, 53(2), 158-164. doi.org/10.1080/00405841.2014.885814.

Capra, F. (1997). The web of life: A new scientific understanding of living systems. New York, NY: Anchor.

Capra, F. (2008). The science of Leonardo. New York: Anchor Books.

Capra, F. (2016). The systems View of Life Course. Totnes, England: Schumacher College.

Capra, F., \& Luisi, P. (2016). The systems view of life: A unifying vision. Cambridge UK: Cambridge University Press.

Capra, F., \& Mattei, U. (2015). The ecology of law: Toward a legal system in tune with nature and community. Oakland, CA: Berrett-Koehler.

Capra, F., \& Pauli, G. (Eds.). (1995). Steering business toward sustainability. Tokyo: United Nations University Press.

Carpenter, V., \& Osborne, S. (2014). Twelve thousand hours: Education and poverty in Aotearoa New Zealand. Auckland, NZ: Dunmore Publishing Limited.

Carr, W., \& Kemmis, S. (1986). Becoming critical: Education knowledge and action research. London: The Falmer Press.

Castells, M. (2009). Communication power. Oxford: Oxford University Press.

Chatwani, N. (2017). Distributed leadership: The dynamics of balancing leadership with followership. Cham, Switzerland: Springer Nature.

Cherkowski, S. (2010). Leadership for diversity, inclusion and sustainability: Teachers as leaders. Citizenship, Social and Economic Education, 9(1), 2331.

Cherkowski, S., \& Ragoonaden, K. (2016). Leadership for diversity: Intercultural communication competence as professional development. Teacher Learning and Professional Development, 1 (1), 33-43. Retrieved from http://journals.sfu.ca/tlpd/index.php/tlpd/article/view/5.

Children's Commissioner (2018). Education matters to me: Progress and achievement. Detailed report 5 of 6. Wellington: New Zealand School Trustees Association.

Christie, C., Montrosse, B., \& Klein, B. (2005). Emergent design evaluation: A case study. Evaluation and Program Planning, 28(3), 271-277. doi.org/10.1016/j.evalprogplan.2005.04.002. 
Cilliers, P. (2002). Complexity and postmodernism: Understanding complex systems. London: Routledge.

Claxton, G. (2005). An intelligent look at emotional intelligence: A publication commissioned by the Association of Teachers and Lecturers. London: ATL.

Claxton, G., Costa, A., \& Kallick, B. (2016). Hard thinking about soft kills. Educational Leadership. 73(6),60-64.

Coelli, M., \& Green, D. A. (2012). Leadership effects: School principals and student outcomes. Economics of Education Review, 31(1), 92-109.

Cohen, L., Manion, L., \& Morrison, K. (2017). Research methods in education $\left(^{\text {th }}\right.$ ed.). London, UK: Taylor and Francis Limited.

Cordon, S. L., Brown, K. W., \& Gibson, P. R. (2009). The role of mindfulness-based stress reduction on perceived stress: Preliminary evidence for the moderating role of attachment style. Journal of Cognitive Psychotherapy, 23(3), 258-269. doi.org/10.1891/0889-8391.23.3.258.

Cotton, K. (2003). Principals and student achievement: What the research says. Alexandria, VA: Association for Supervision and Curriculum Development (ASCD). Retrieved from http://www.eric.ed.gov/ERICWebPortal/detail?accno=ED482257.

Craig, W., Pepler, D., \& Blais, J. (2007). Responding to bullying: What works? School Psychology International, 28(4), 465-477. doi.org/10.1177/0143034307084136

Creswell, J. W., Hanson, W. E., Clark Plano, V. L., \& Morales, A. (2007). Qualitative research designs: Selection and implementation. The Counseling Psychologist, 35(2), 236-264. doi.org/10.1177/0011000006287390.

Crippen, C. (2004). Servant-leadership as an effective model for educational leadership and management: First to serve, then to lead. Management in Education, 18(5), 11-16.

Dalcher, D. (2018). The Wisdom of teams revisited: Teamwork, teaming and working for the common good. PM World Journal, 7(9), September 2018

Davies, B., \& Harré, R. (1990). Positioning: The discursive production of selves. Journal for the Theory of Social Behaviour, 20(1), 43-63.

Davis, B., \& Sumara, D. (2006). Complexity and education: Inquiries into learning, teaching, and research. Mahwah, NJ: Routledge.

Davis, B., Sumara, D., \& Luce-Kapler, R. (2015). Engaging minds: Cultures of education and practices of teaching ( $3^{\text {rd }} \mathrm{ed}$.). New York: Routledge. 
Day, C. (2005). Principals who sustain success: Making a difference in schools in challenging circumstances. International Journal of Leadership in Education, 8(4), 273-290. doi.org/10.1080/13603120500330485.

Day, C. (2014). Resilient principals in challenging schools: The courage and costs of conviction. Teachers and Teaching, 20(5), 638-654.

Day, C. (2017). Teachers' worlds and work: Understanding complexity, building quality. London: Routledge.

Day, C., Gu, Q., \& Sammons, P. (2016). The impact of leadership on student outcomes: How successful school leaders use transformational and instructional strategies to make a difference. Educational Administration Quarterly, 52(2), 221-258.

Deal, T. E., \& Kennedy, A. A. (1983). Culture: A New Look Through Old Lenses. The Journal of Applied Behavioural Science, 19(4), 498-505.

Deal, T., \& Peterson, K. (2016). Shaping School Culture / Terrence E. Deal, Kent D. Peterson.

Denis, J.-L., Langley, Ann., \& Rouleau, L. (2010). The practice of leadership in the messy world of organizations. Leadership, 6 (1), 67-88. https://doi.org/10.1177/1742715009354233.

Denzin, N. K. (2009). The research act: A theoretical introduction to sociological methods. Somerset, UK: Taylor and Francis.

Denzin, N. K., \& Lincoln, Y. S. (2011). The SAGE handbook of qualitative research. Thousand Oaks, United States: Sage Publications Limited.

Department of Education Task Force (1988) Administering for excellence. Effective administration in education. Wellington: Government Printer.

Despres, B. R. (2004). Systemic thinking and educational leadership: Some considerations. International Electronic Journal for Leadership in Learning, 8 (n.p). Retrieved from

http://iejll.journalhosting.ucalgary.ca/iej11/index.php/ijll/article/view/700.

Dewey, J. (1904). The Relation of Theory to Practice in Education. In C. A. McMurry (Ed.), The Third Yearbook of the National Society for the Scientific Study of Education. Part I. (pp. 9-30). Chicago, IL: The University of Chicago Press. Retrieved from https://archive.org/details/r00elationoftheorynatirich.

Dewey, J. (2004). Democracy and education: An introduction to the philosophy of education. New York, United States: Dover Publications Inc. 
Dufour, R., \& Marzano, R. J. (2011). Leaders of learning: How district, school, and classroom leaders improve student achievement. Bloomington, IN: Solution Tree.

Duignan, P. (2012). Educational leadership: Together creating ethical learning environments. Cambridge, UK: Cambridge University Press.

Duncan, G. J., \& Murnane, R. J. (2011). Whither opportunity? Rising inequity, schools, and children's life chances. United States: Russell Sage Foundation.

Dunne, J. (1993). Back to the rough ground: "phronesis" and "techne" in modern philosophy and in Aristotle. Notre Dame: University of Notre Dame Press.

Durr, M., \& Hill, S. (2006). Race, work, and family in the lives of African Americans. United States: Rowman \& Littlefield.

Eckman, E., \& Kelber, S. (2010). Female traditional principals and co-principals: Experiences of role conflict and job satisfaction, Journal of Educational Change 11(3), 205-219.

Education Council of New Zealand. (2017). Code of professional responsibility and standards for the teaching profession. Wellington: Education Council, New Zealand.

Education Review Office. (2010). Promoting success for Māori students: Schools' progress. Wellington, NZ: Education Review Office. Retrieved from http://www.ero.govt.nz/National-Reports/Promoting-Success-for-MāoriStudents-Schools-Progress-June-2010/Overview.

Education Review Office. (2016). School evaluation indicators: Effective practice for improvement and learner success. New Zealand: Crown Copyright.

Eliophotou Menon, M. (2014). The relationship between transformational leadership, perceived leader effectiveness and teachers' job satisfaction. Journal of Educational Administration, 52(4), 509-528. doi.org/10.1108/JEA-01-20130014.

Elmore, R. F. (1979). Backward mapping: Implementation research and policy decisions. Political Science Quarterly, 94(4), 601. doi.org/10.2307/2149628.

Elmore, R. F. (2000). Building a new structure for school leadership. Washington DC: Albert Shanker Institute. Retrieved from https://eric.ed.gov/?id=ED546618.

Enomoto, E., Kramer, B., \& Starratt, R. J. (2007). Leading through the quagmire: Ethical foundations, critical methods, and practical applications for school leadership. Lanham, MD: Rowman \& Littlefield Education. 
Eriksson, M., Ghazinour, M., \& Hammarström, A. (2018). Different uses of

Bronfenbrenner's ecological theory in public mental health research: What is their value for guiding public mental health policy and practice? Social Theory \& Health, 16(4), 414-433. doi.org/10.1057/s41285-018-0065-6.

Ferriter, W. M., \& Cancellieri, P. J. (2017). Creating a culture of feedback. Bloomington IN: Solution Tree Press.

Firestone, W. A., \& Riehl, C. (2005). A new agenda for research in educational leadership. New York: Teachers College Press.

Fitzsimons, D., James, K. T., \& Denyer, D. (2011). Alternative approaches for studying shared and distributed leadership: Studying shared and distributed leadership. International Journal of Management Reviews, 13(3), 313-328. doi.org/10.1111/j.1468-2370.2011. 00312.x.

Flick, U. (1992). Triangulation revisited: Strategy of validation or alternative? Journal for the Theory of Social Behaviour, 22(2), 175-197. doi.org/10.1111/j.1468-5914. 1992.tb00215. x.

Flick, U. (2018). Doing triangulation and mixed methods ( $2^{\text {nd }}$ ed.). London: Sage Publications Limited.

Ford, T. (2010). Examining Culturally Responsive Leadership: An investigation into how one school leader, in a primary school setting, provides culturally responsive leadership that ensures Maori students achieve (Thesis, Master of Educational Leadership (MEd Leadership). University of Waikato, Hamilton, New Zealand. Retrieved from https://hdl.handle.net/10289/5594.

Friedrich, T., Griffith, J. A., \& Mumford, M. (2016). Collective leadership behaviors: Evaluating the leader, team network, and problem situation characteristics that influence their use. The Leadership Quarterly, 27(2), 312333.

Fullan, M. (1982). The meaning of educational change. New York, NY: Retrieved from http://www.eric.ed.gov/ERICWebPortal/detail?accno=ED218247.

Fullan, M. (2003). The moral imperative of school leadership. United States: Sage Publications Limited.

Fullan, M. (2005). Leadership and sustainability: System thinkers in action. Thousand Oaks, CA: Corwin Press.

Fullan, M. (2006). Quality leadership, quality learning: Proof beyond reasonable doubt. Chapter prepared for the Irish Primary Principals' Network. Cork: Ireland Lionra+. 
Fullan, M. (2007). Leading in a culture of change (Rev. ed.). San Francisco: JosseyBass.

Fullan, M. (2009). The challenge of change: Start school improvement now! $2^{\text {nd }}$ ed.). Thousand Oaks, CA: Corwin.

Fullan, M. (2011). Change leader: Learning to do what matters most. San Francisco: Jossey-Bass/Wiley.

Fullan, M. (2018). The principalship has changed:2020 here we come! Principals Connections. 22(1),18-19.

Fullan, M., Hill, P. W., \& Crévola, C. (2006). Breakthrough. Thousand Oaks, CA: Corwin Press.

Gall, M., Gall, J., \& Borg, W. (2007). Educational research: An introduction ( $8^{\text {th }}$ ed.). Boston: Pearson/Allyn \& Bacon.

Gallagher, M. (2008). Foucault: Power and participation. The International Journal of Children's Rights, 16(3), 395-406. Netherlands: Brill Publishing.

Garvey Berger, J. (2012). Cultivating wisdom. Leader to Leader. 66 (Fall), 40-44.

Gegeo, D. W., \& Watson-Gegeo, K. A. (2002). Whose knowledge? Epistemological collisions in Solomon Islands community development. Retrieved from http://scholarspace.manoa.hawaii.edu/handle/10125/13653.

Geyer, R., \& Cairney, P. (2015). Handbook on complexity and public policy. New Jesey, United States: Edward Elgar Publishing.

Gibbs, G. R. (2012). Different approaches to coding. Sociological Methodology, 42(1), 82-84. doi.org/10.1177/0081175012460853.

Gibson, A. (2014). Principals' and teachers' views of spirituality in principal leadership in three primary schools. Educational Management Administration \& Leadership, 42(4), 520-535. doi.org/10.1177/1741143213502195.

Gilbert, J. (2005). Catching the knowledge wave: The knowledge society and the future of education. Wellington: New Zealand Council for Educational Research Press.

Giles, D. \& Palmer, C. (2015). Exploring a Principal's Practice during a Period of Significant Organizational Change: Relational Leadership and Sensibilities in Action. The Journal of Meaning-Centred Education, 3(1), n.p.

Glasser, W. (1999). Choice theory: A new psychology of personal freedom. New York, USA: HarperCollins Publishers Inc.

Glickman, C., Gordon, S., \& Ross-Gordon, J. (2004). Supervision and instructional leadership: A developmental approach. Boston, Mass: Allyn and Bacon. 
Goldspink, C. (2007). Rethinking educational reform: A loosely coupled and complex systems perspective. Educational Management Administration \& Leadership, 35(1), 27-50. doi.org/10.1177/1741143207068219.

Goldstein, J., Hazy, J. K., \& Lichtenstein, B. B. (2010). Complexity and the nexus of leadership. New York: Palgrave Macmillan. Retrieved from http://link.springer.com/10.1057/9780230107717.

Goleman, D., \& Senge, P. (2014). The triple focus: A new approach to education. Northampton: More Than Sound.

Goleman, D., Boyatzis, R. E., \& McKee, A. (2013). Primal leadership, with a new preface by the authors: Unleashing the power of emotional intelligence. Boston, MA: Harvard Business Review Press.

Gordon, E. W., Jean-Louis, B., \& Obiora, N. (Eds.). (2017). Strengthening families, communities, and schools to support children's development: Neighbourhoods of promise. New York: Routledge.

Govia, M. (2017). An investigation of leadership practices that yield success in renewal schools. Available from ProQuest Dissertations \& Theses Global. Retrieved from https://search-proquestcom.helicon.vuw.ac.nz/docview/1975374274?accountid=14782.

Grenda, J. P., \& Hackmann, D. G. (2014). Advantages and challenges of distributing leadership in middle-level schools. NASSP Bulletin, 98(1), 53-74.

Gronn, P. (2003). The new work of educational leaders: Changing leadership practice in an era of school reform. Thousand Oaks: Sage.

Gronn, P. (2009). Leadership configurations. Leadership, 5(3), 381-394. doi.org/10.1177/1742715009337770.

Grootenboer, P., \& Hardy, I. (2017). Contextualizing, orchestrating and learning for leading. Educational Management Administration \& Leadership, 45(3), 402418. doi.org/10.1177/1741143215595418.

Groysberg, B., \& Slind, M. (2012). Talk, Inc: How trusted leaders use conversation to power their organizations. Boston, MA: Harvard Business Review Press.

Guba, E. G., \& Lincoln, Y. S. (2005). Paradigmatic controversies, contradictions, and emerging confluences. In N.K. Denzin \& Y. Lincoln (Eds.), The Sage handbook of qualitative research ( $3^{\text {rd }}$ ed., pp. 191-215). Thousand Oaks, CA: Sage Publications Limited.

Gunter, H. (2001). Critical approaches to leadership in education. The Journal of Educational Enquiry, 2(2). 94-108. 
Gutiérrez, R. (2008). A “gap-gazing” fetish in mathematics education?

Problematizing research on the achievement gap. Journal for Research in Mathematics Education, 39(4), 357-364.

Hallinger, P. (2003). Leading educational change: Reflections on the practice of instructional and transformational leadership. Cambridge Journal of Education, 33(3), 329-352. doi.org/10.1080/0305764032000122005.

Hallinger, P. (2011). Leadership for learning: Lessons from 40 years of empirical research. Journal of Educational Administration, 49(2), 125-142. doi.org/10.1108/09578231111116699.

Hallinger, P. (2016). Bringing context out of the shadows of leadership. Educational Management Administration \& Leadership, 46(1), 5-24. doi.org/10.1177/1741143216670652.

Hannon, V., \& Peterson, A. (2017). Thrive: Schools reinvented for the real challenges we face 1. London: Innovation Unit Press.

Hargreaves, A., \& Fullan, M. (1998). What's worth fighting for out there? New York: Teachers College Press.

Harris, A. (2003). Building leadership capacity for school improvement. London: McGraw Hill Education (UK).

Harris, A. (2009). Distributed leadership: What we know. In P. A. Harris (Ed.), Distributed leadership (pp. 11-21). Netherlands: Springer. Retrieved from http://link.springer.com/chapter/10.1007/978-1-4020-9737-9_2.

Harris, A., \& Spillane, J. (2008). Distributed leadership through the looking glass. Management in Education, 22(1), 31-34. doi.org/10.1177/0892020607085623.

Heck, D., \& Weiss, I. (2005). Strategic Leadership for Education Reform: Lessons from the Statewide Systemic Initiatives Program. Consortium for Policy Research and Education. USA.

Heifetz, R. A., Grashow, A., \& Linsky, M. (2009). The practice of adaptive leadership: Tools and tactics for changing your organization and the world. Boston, MA: Harvard Business Press.

Hess, F. M., \& Meeks, O. M. (2010). Unbundling schools. Phi Delta Kappan, 92(3), 41-42. doi.org/10.1177/003172171009200312.

Hiller, N., Day, D., \& Vance, R. (2006). Collective enactment of leadership roles and team effectiveness: A field study. The Leadership Quarterly, 17, 387-397. doi.org/10.1016/j.leaqua.2006.04.004. 
Hohepa, M. K., \& Lloyd, C. (2008). Māori and educational leadership: Tu rangatira. Paper presented at the 2008 Annual Meeting of the American Educational Research Association, New York.

Holsted, I. (2018). Māori student under-achievement "chronic.” Retrieved from https://www.radionz.co.nz/national/programmes/morningreport/audio/201863 2155/Māori-student-under-achievement-chronic.

Hooper, M. A., \& Bernhardt, V. L. (2016). Creating capacity for learning and equity in schools: Instructional, adaptive, and transformational leadership. New York: Routledge.

Hoppey, D., \& McLeskey, J. (2013). A case study of principal leadership in an effective inclusive school. The Journal of Special Education, 46(4), 245-256. doi.org/10.1177/0022466910390507.

Hoque, A. (2018). Third-generation British-Bangladeshis from east London:

Complex identities and a culturally responsive pedagogy. British Journal of Sociology of Education, 39(2), 182-196.

Houghton, C. (2015). Underachievement of Maori and Pasifika learners and culturally responsive assessment. Journal of Initial Teacher Inquiry. 1(1), 10-12.

Hulpia, H., Devos, G., \& Rosseel, Y. (2009). The relationship between the perception of distributed leadership in secondary schools and teachers' and teacher leaders' job satisfaction and organizational commitment. School Effectiveness and School Improvement, 20(3), 291-317.

Hurren, B. L. (2006). The effects of principals' humor on teachers' job satisfaction. Educational Studies, 32(4), 373-385. doi.org/10.1080/03055690600850321.

Huxley, A. (1954). The doors of perception. UK: Chatto \& Windus.

Ibara, E. (2010). Perspectives in educational administration. Port Harcourt, Nigeria: Rodi.

Jackson, B., Nicoll, M., \& Roy, M. J. (2017). The distinctive challenges and opportunities for creating leadership within social enterprises. Social Enterprise Journal, 14(1), 71-91. doi.org/10.1108/SEJ-03-2017-0016.

Jacobson, S. L., Brooks, S., Giles, C., Johnson, L., \& Ylimaki, R. (2007). Successful leadership in three high-poverty urban elementary schools. Leadership and Policy in Schools, 6(4), 291-317. doi.org/10.1080/15700760701431553.

Johnson, L. D. (2012). Culturally responsive leadership. In J. Banks (Ed.), Encyclopedia of diversity in education. Thousand Oaks, CA: Sage Publications Limited. 
Jones, S., Lefoe, G., Harvey, M., \& Ryland, K. (2012). Distributed leadership: A collaborative framework for academics, executives and professionals in higher education. Journal of Higher Education Policy and Management, 34(1), 67-78. doi.org/10.1080/1360080X.2012.642334.

Kania, J., \& Kramer, M. (2011). Collective impact. Stanford Social Innovation Review, 9(1), 36-41.

Kekana, D. (2013). The assumptions, values and beliefs of school principals regarding school leadership and management. Pretoria: South Africa University of Pretoria.

Kemp, A. (2015). Expectations and reality: Primary school principals' experiences of change leadership in the transition to digital learning environments. Auckland: Unitec Institute of Technology.

Kempster, S., Parry, K., \& Jackson, B. (2016). Methodologies to discover and challenge leadership-as-practice. In J. A. Raelin (Ed.), Leadership-aspractice: theory and application (pp. 242-261). New York: Routledge Studies in Leadership Research.

Khan, S., \& VanWynsberghe, R. (2008). Cultivating the Under-Mined: Cross-Case Analysis as Knowledge Mobilization. Forum Qualitative Sozialforschung / Forum: Qualitative Social Research, 9(1). doi: http://dx.doi.org/10.17169/fqs-9.1.334.

Klare, M. (2012). The race for what's left: The global scramble for the world's last resources. New York, NY: Picador.

Klimek, K. J., Ritzenhein, E., \& Sullivan, K. D. (2008). Generative leadership: Shaping new futures for today's schools. Thousand Oaks, CA: Corwin Press. Knyazeva, H. (1999). Synergetics and the images of future. Futures, 31(3), 281-290. Kocolowski, M. D. (2010). Shared leadership: Is it time for a change. Emerging Leadership Journeys, 3(1), 22-32.

Krachman, S., Arnold, R., \& Larocca, R. (2016). The CORE Districts Expanding the definition of student success under ESSA. United States: Transforming Education.

Kramer, B. H., Enomoto, E., \& Enomoto, E. (2014). Leading ethically in schools and other organizations: Inquiry, case studies, and decision-making. Lanham, MD: Rowman \& Littlefield.

Kutz, M. (2016). Contextual intelligence: Smart leadership for a constantly changing world. Ohio: Roundtable Group, LLC. 
Labour government. (n.d). Labours vision for education.

Ladd, H. F. (2012). Education and poverty: Confronting the evidence. Journal of Policy Analysis and Management, 31(2), 203-227.

Ladson-Billings, G. (2006). From the achievement gap to the education debt: Understanding achievement in U.S. schools. Educational Researcher, 35(7), 3-12. doi.org/10.3102/0013189X035007003.

Ladson-Billings, G. (2014). Culturally Relevant Pedagogy 2.0: A.k.a. the Remix. Harvard Educational Review, 84(1), 74-135.

Lambert, L. (2002a). Building leadership capacity in schools. Australia: The Australian Principals Centre.

Lambert, L. (2002b). A framework for shared leadership. Educational Leadership, 59(8), 37-40.

Lambert, L. (2003a). Leadership capacity for lasting school improvement. Alexandria, VA. ASCD.

Lambert, L. (2003b). Shifting conceptions of leadership: Towards a redefinition of leadership for the twenty-first century. Handbook of educational leadership and management. (pp. 5-15). West-Burnham: Pearson Education.

Lambert, L., Walker, D., Zimmerman, D., Cooper, J., Lambert, M., Gardner, M., \& Szabo, M. (2002). The constructivist leader ( $2^{\text {nd }}$ ed.). New York, NY: Teachers College Press.

Lárusdóttir, S. (2014). Educational leadership and market values: A study of school principals in Iceland. Educational Management Administration \& Leadership, 42(4_suppl), 83-103.https://doi.org/10.1177/1741143213510499

Leithwood, K., Harris, A., \& Hopkins, D. (2008). Seven strong claims about successful school leadership. School Leadership \& Management, 28(1), 2742. doi.org/10.1080/13632430701800060.

Leithwood, K., Jantzi, D., \& Steinbach, R. (1999). Changing leadership for changing times. Philadelphia: Open University Press.

Lemke, J. L., \& Sabelli, N. H. (2008). Complex systems and educational change: Towards a new research agenda. Educational Philosophy and Theory, 40(1), 118-129. doi.org/10.1111/j.1469-5812.2007. 00401.x.

Levin, H. (2006). Can research improve educational leadership? Educational Researcher, 35(8), 38-43. Doi.org/10.3102/0013189X035008038.

Lewin, K. (1976). Field theory in social science: Selected theoretical papers. Chicago: University of Chicago Press. 
Lewin, K. (1997). Resolving social conflicts and field theory in social science:

Washington, DC: American Psychological Association.

Louis, K., \& Murphy, J. (2018). Trust, caring and organisational leaning: The leader's role, Journal of Educational Administration, 55 (1), 1030126.

Lowenhaupt, R. (2014). The language of leadership: Principal rhetoric in everyday practice. Journal of Educational Administration, 52(4), 446-468. doi.org/10.1108/JEA-11-2012-0118.

Lumby, J., \& English, F. (2009). From simplicism to complexity in leadership identity and preparation: Exploring the lineage and dark secrets. International Journal of Leadership in Education, 12(2), 95-114. doi.org/10.1080/13603120802449678.

Lupton, R. (2005). Social justice and school improvement: Improving the quality of schooling in the poorest neighbourhoods. British Educational Research Journal, 31(5), 589-604. doi.org/10.1080/01411920500240759.

Lyman, L. L., Ashby, D. E., \& Tripses, J. S. (2005). Leaders who dare: Pushing the Boundaries. Maryland: Rowman and Littlefield Education.

MacNeill, N., Silcox, S., \& Boyd, R. (2018). Transformational and transactional leadership: A false dichotomy of leadership in schools. Education Today, $18(1)$ 10-12.

Mac Ruairc, G., Ottesen, E., \& Precey, R. (2013). Leadership for inclusive education. Netherlands: Sense publishers.

Madden, K. (2014). Matching passion with precision for struggling New Zealanders. The heart of poverty series (No. 1). Retrieved from https://www.maxim.org.nz/heart-poverty-1/.

Manu'atu, L. (2000). Tuli Ke Ma'u Hono Ngaahi Mālie: Pedagogical possibilities for Tongan students in New Zealand secondary schooling. Auckland: University of Auckland. Retrieved from https://www.researchgate.net/publication/37985247_Tuli_Ke_Ma\%27u_Hon o_Ngaahi_Malie_Pedagogical_possibilities_for_Tongan_students_in_New_Z ealand_secondary_schooling.

Marion, R., \& Uhl-Bien, M. (2001). Leadership in complex organizations. Retrieved from http://digitalcommons.unl.edu/managementfacpub/11.

Martin, M. (2010). Leading learning - By Tom O’Donoghue \& Simon Clarke. British Journal of Educational Technology, 41(3), E64-E64. doi.org/10.1111/j.1467-8535.2010.01080_9.x. 
Marzano, R. J. (2005). School leadership that works: From research to results.

Alexandria, VA: Aurora, CO: Mid-continent Research for Education and Learning, Association for Supervision \& Curriculum Development.

Marzano, R. J., Waters, T., \& McNulty, B. A. (2005). School leadership that works: From research to results. Alexandira, VA: ASCD.

Mascolo, L. B. (2014). Leading through laughter: Humor and perceived effectiveness of $P-12$ principals. Georgia Southern University, Statesboro, Georgia.

Mason, M. (Ed.). (2008). Complexity theory and the philosophy of education. Chichester, West Sussex, UK; Wiley-Blackwell.

Maykut, P. S., \& Morehouse, R. E. (1994). Beginning qualitative research: A philosophic and practical guide. London: The Falmer Press.

Mcfarlane, A., \& Derby, M (2018). Mai i Te Ao Tawhito Ki Te Ao Tūroa: Education Leaders as Cultural Advocates. Ipu Kererū, 16 Jan. 2018, nzareblog.wordpress.com/2018/01/17/macfarlane-ed-leaders/.

McGee, A., Haworth, P., \& MacIntyre, L. (2014). Leadership practices to support teaching and learning for English language learners. TESOL Quarterly, 49(1), 92-114. doi.org/10.1002/tesq.162.

McGee, E. O. (2013). Threatened and placed at risk: High achieving African American males in urban high schools. The Urban Review, 45(4), 448-471. doi.org/10.1007/s11256-013-0265-2.

McIntosh, G., \& S., Rima. (2007). Overcoming the dark side of leadership: How to become an effective leader by confronting potential failures. Ada, MI: Baker Publishing Group.

McKague, K., Wheeler, D., \& Karnani, A. (2015). An integrated approach to poverty alleviation: Roles of the private sector, government and civil society. In V. Bitzer, R. Hamann, M. Hall, \& E. W. Griffin-EL (Eds.), The business of social and environmental innovation (pp. 129-145). Switzerland: Springer International Publishing.

McKinley, E., \& Hoskins, T. (2011). Māori education and achievement. In T. McIntosh \& M, Mulholland. Maori and social issues. Wellington: Huia.

McNae, R., Morrison, M., \& Notman, R. (2017). Educational leadership in Aotearoa New Zealand: Issues of context and social justice. Wellington: NZCER Press. Merriam, S. B. (1998). Qualitative research and case study applications in education. San Francisco: Jossey- Bass Publishers. 
Mertens, D. M. (2014). Research and evaluation in education and psychology: Integrating diversity with quantitative, qualitative, and mixed methods. Thousand Oaks, California: Sage Publications Inc.

Meyer, L., \& Evans, I. (2012). The school leader's guide to restorative school discipline / Luanna H. Meyer, Ian M. Evans. Thousand Oaks, Calif.: Corwin.

Miles, M. B., Huberman, A. M., \& Saldana, J. (2013). Qualitative Data Analysis. Thousand Oaks, California: Sage Publications Limited.

Miller, P. (2018). 'Culture', 'Context', School Leadership and Entrepreneurialism: Evidence from Sixteen Countries. Education Sciences 8, (76), 2-14. doi:10.3390/educsci8020076.

Mills, A., Durepos, G., \& Wiebe, E. (2010). Encyclopaedia of case study research. Thousand Oaks, CA. Sage Publications Limited.

Ministry of Education. (1998). New Zealand schools 1997. Wellington: Learning Media.

Ministry of Education. (2007). The New Zealand curriculum. Wellington: Learning Media.

Ministry of Education. (2008). Kiwi leadership for principals: Principals as educational leaders. Wellington: Ministry of Education.

Ministry of Education. (2009). Reading and writing standards for years 1-8. Wellington: Ministry of Education.

Ministry of Education. (2010). Tū rangatira: Maori medium educational leadership. Wellington: Huia.

Ministry of Education. (2013). Ka hikitia: Accelerating success, 2013-2017.

Wellington: Ministry of Education.

Ministry of Education. (2015). Investing in Educational Success Policy. Wellington: Ministry of Education.

Ministry of Education. (2018). Education Counts. Children Living in Low Income

Households. Retrieved from www.educationcounts.govt.nz/find-school.

Morin, E., \& Montuori, A. (2008). On complexity. Cresskill, NJ: Hampton Press.

Morrison, K. (2010). Complexity theory, school leadership and management:

Questions for theory and practice. Educational Management Administration \& Leadership, 38(3), 374-393. doi.org/10.1177/1741143209359711.

Moshayedi, S. (2018). Elementary Principal Leadership and Learning Outcomes for Low Socioeconomic Status Hispanic English Learner. Dissertation (Ed. D.), University of Southern California: California, United States of America. 
Mulcahy, D. G. (Ed.). (2013). Transforming schools: Alternative perspectives on school reform. Charlotte, NC: Information Age.

Naglieri, J., \& Goldstein, S. (2011). Encyclopaedia of child behaviour and development. New York: Springer.

Nicolis, G., \& Prigogine, I. (1977). Self-organization in nonequilibrium systems: From dissipative structures to order through fluctuations. New York, NY: Wiley.

Niesche, R. (2016). Perpetuating inequity in education: Valuing purpose over process in educational leadership. In J, Brooks \& A Normore (Eds.), The dark side of leadership: Identifying and overcoming unethical practice in organizations. Bingley, UK: Emerald Group Publishing Limited.

Nieuwenhuis, J., Beckmann, J., \& Prinsloo, I. (Eds.). (2007). Growing human rights and values in education. Pretoria, South Africa: Van Schaik Publishers.

Normore, A., \& Brooks, J. (Eds.). (2017). The dark side of leadership: identifying and overcoming unethical practice in organizations. Bingley, UK: Emerald.

Notman, R. (2011). Successful educational leadership in New Zealand case studies of schools and an early childhood centre. Wellington, NZ: New Zealand Council for Educational Research Press.

Notman, R. (2017). Holistic leadership in a high-needs early childhood centre. In R. McNae, M. Morrison \& R. Notman (Eds.), Educational leadership in Aotearoa New Zealand: Issues of context and social justice. (pp. 130-142). Wellington, New Zealand: NZCER Press.

O’Donoghue, T., \& Clarke, S. (2009). Leading learning: process, themes and issues in international contexts. London: Routledge.

OECD, (1996). The knowledge-based economy. Paris: OECD

OECD (Ed.). (2012). Equity and quality in education: supporting disadvantaged students and schools. Paris: OECD.

OECD. (2015). PISA 2015 results (Volume I): Excellence and equity in education en - OECD. Retrieved from http://www.oecd.org/publications/pisa-2015results-volume-i-9789264266490-en.htm.

OECD. (2018). Equity in education: Breaking down barriers to social mobility. Paris: PISA, OECD Publishing.

Oghenejobo, M. (2014). Strategic leadership for sustainable personal and organizational success. Bloomington, IN: WestBow Press. 
Ospina, S., \& Sorensen, G. (2006). A constructionist lens on leadership: Charting new territory. In G. Goethals, \& G. Sorenson (Eds.), In quest of a general theory of leadership (pp. 188-204). Cheltenham, UK: Edward Elgar Publishers.

Ovens, A., Hopper, T., \& Butler, J. (Eds.) (2013). Complexity Thinking in Physical Education: Reframing Curriculum, Pedagogy and Research. London: Routledge.

Parata, H., Joyce, H., Clark, B., \& Benny, D. (2013). Pasifika Education Plan 2013: 2017. Wellington: Ministry of Education.

Parrett, W., \& Budge, K. M. (2012). Turning high-poverty schools into highperforming schools. Alexandria, VA: Association for Supervision \& Curriculum Development.

Pashiardis, P., \& Notman, R. (2012). Intrapersonal factors in New Zealand school leadership success. International Journal of Educational Management, 26(5), 470-479. Bingley, UK: Emerald Group Publishing Limited.

Patton, M. Q. (1990). Qualitative evaluation and research methods (2 ${ }^{\text {nd }}$ ed.). Thousand Oaks, CA: Sage Publications Limited.

Patton, M. Q. (2002). Qualitative research \& evaluation methods. Thousand Oaks, United States: Sage Publications Limited.

Paul, R., \& Elder, L. (2013). Critical thinking: Tools for taking charge of your learning and your life. NJ, United States: Pearson Education.

Professional Development Advisory Group. (2014). Report of the professional development advisory group. Wellington: Ministry of Education.

Pejza, J. P. (1985). The Catholic school principal: A different kind of leader. Retrieved from https://eric.ed.gov/?id=ED256053.

People for Education. (2013). Broader measures of success: Measuring what matters in education. Canada: A People for Education Project.

Pepper, K. (2010). Effective principals skilfully balance leadership styles to facilitate student success: A focus for the reauthorization of ESEA. Planning and Changing, 41(1/2), 42-56. Illinois: Department of Educational Administration and Foundations.

Pick, J. B., \& Sarkar, A. (2015). The global digital divides: Explaining Change. Berlin: Springer.

Pink. (2008). A whole new mind: Moving from the information age to the conceptual age. New York: Penguin Putnam Incorporated. 
Pirbhai-Illich, F., Pete, S., \& Martin, F. (2017). Culturally Responsive Pedagogy Working towards Decolonization, Indigeneity and Interculturalism / Edited by Fatima Pirbhai-Illich, Shauneen Pete, Fran Martin.

Portelli, J. P., \& Campbell-Stephens, R. (2009). Leading for equity: The investing in diversity approach. Toronto: Edphil Books.

Portin, B. S. (2000). The changing urban principalship. Education and Urban Society, 32(4), 492-505.

PPTA. (2013). NZ Schools: The decile system. Issues and organising seminar. Wellington: PPTA.

Prigogine, I. (1997). The End of Certainty by Ilya Prigogine. Free Press.

Raelin, J. A. (2016). Leadership-as-practice: Theory and application. New York: Routledge. Retrieved from http://helicon.vuw.ac.nz/login?url=http://search.ebscohost.com/login.aspx?dir ect $=$ true $\& d b=$ nlebk $\& A N=1163621 \&$ site $=$ ehost-live.

Ragnedda, M., \& Muschert, G. W. (2013). The digital divide: The internet and social inequity in international perspective. London and New York: Routledge.

Rashbrooke, M. (2013). Inequity: A New Zealand crisis. Wellington: Bridget Williams Books Limited.

Reed, L. C., \& Swaminathan, R. (2014). An urban school leader's approach to school improvement toward contextually responsive leadership. Urban Education. doi.org/10.1177/0042085914553675.

Reeves, D. (2008). Reframing teacher leadership to improve your school. Alexandria, VA: Association for Supervision \& Curriculum Development. Reyes, P., Scribner, J. D., \& Scribner, A. P. (1999). Lessons from high-performing Hispanic schools: Creating learning communities. Williston, VT: Teachers College Press.

Rice, J. K. (2010). Principal effectiveness and leadership in an era of accountability: What research says (Brief 8). Washington DC: National Center for Analysis of Longitudinal Data in Education Research. Retrieved from https://eric.ed.gov/?id=ED509682.

Richardson, K. (2004). The problematisation of existence: towards a philosophy of complexity. Nonlinear Dynamics, Psychology, and Life Sciences, 8(1), 17-40.

Robertson, J. (2016). Coaching leadership: Building educational leadership capacity through partnership ( $2^{\text {nd }}$ ed.). Wellington: New Zealand Council for Educational Research. 
Robertson, S. (2017). Transformation of professional identity in an experienced primary school principal: A New Zealand case study. Educational Management Administration \& Leadership, 45(5), 774-789. doi.org/10.1177/1741143217707519.

Robinson, D. (2004). Unconditional leadership: A principle-centred approach to developing people, building teams and maximising results. London: Community Links.

Robinson, V. M. J., Lloyd, C. A., \& Rowe, K. J. (2008). The impact of leadership on student outcomes: An analysis of the differential effects of leadership types. Educational Administration Quarterly, 44(5), 635-674. doi.org/10.1177/0013161X08321509.

Robinson, V. M., Hohepa, M. K., \& Lloyd, C. (2009). School leadership and student outcomes: Identifying what works and why: Best evidence synthesis iteration (BES). Wellington. NZ: Ministry of Education.

Robinson, J., \& Godbey, G. (2000). Time for life: The surprising way Americans use their time $\left(2^{\text {nd }}\right.$ ed.). United States of America: The Pennsylvania State University Press.

Sahlberg. P (2011). Paradoxes of educational improvement: The Finnish experience. Scottish Educational Review, 43 (1), 3-23.

Sahlberg, P. (2018). To rush education reform is to ruin it. Retrieved July 1, 2018, from https://www.radionz.co.nz/national/programmes/ninetonoon/audio/20186478 82/pasi-sahlberg-to-rush-education-reform-is-to-ruin-it.

Sahlberg, P., \& Boce, E. (2010). Are teachers teaching for a knowledge society? Teachers and Teaching: Theory and Practice, 16(1), 31-48.

Santamaría, L. J., \& Santamaría, A. P. (2012). Applied critical leadership in education: Choosing change. New York: Routledge.

Schafermeyer, K. (2017). Principals' perceptions of working with suburban elementary students from poverty. Dissertation ( $\mathrm{PhD}$ diss.), Northern Illinois University: Chicago, Illinois.

Schein, S. (2015). A new psychology for sustainability leadership: The hidden power of ecological worldviews. London: Greenleaf Publishing Limited.

Schwandt, T. A. (2000). Three epistemological stances for qualitative inquiry: Interpretivism, hermeneutics, and social constructionism. In Handbook of 
Qualitative Research (Vol. 2, pp. 189-213). Thousand Oaks, CA: Sage Publications Limited.

Sebastian, J., Allensworth, E., \& Huang, H. (2016). The role of teacher leadership in how principals influence classroom instruction and student learning. American Journal of Education, 123(1), 69-108. doi.org/10.1086/688169.

Senge, P. (2006). The fifth discipline: The art and practice of the learning organization (Rev. ed.). New York: Doubleday.

Sergiovanni, T. (1996). Moral leadership: Getting to the heart of school improvement. New York, United States: John Wiley \& Sons Incorporated.

Sergiovanni, T. J. (2003). The life world at the center: Values and action in educational leadership. In N. Bennett, M. Crawford, \& M. Cartwright (Eds.), Effective educational leadership. Thousand Oaks, CA: Sage Publications Limited.

Shaked, H., \& Schechter, C. (2013). Seeing wholes: The concept of systems thinking and its implementation in school leadership. International Review of Education / Internationale Zeitschrift Für Erziehungswissenschaft / Revue Internationale de l'Education, 59(6), 771-791.

Shields, C. M. (2003). Good intentions are not enough: Transformative leadership for communities of difference. Lanham, MD: Scarecrow Press.

Shields, C. M. (2013). Transformative leadership in education: Equitable change in an uncertain and complex world. New York, NY: Routledge.

Shoho, A. R., Barnett, B., \& Cyprès, A. T. (Eds.). (2010). The challenges for new principals in the twenty-first century: Developing leadership capabilities through professional support. Charlotte, N.C: Information Age.

Slomp, D., Mombourquette, C., \& Marynowski, R. (2018). A case study of school leadership and the change process through the lens of Bronfenbrenner's bioecological theory of human development. London: Sage Publications Limited.

Smart, J. (2017). Collective impact: Evidence and implications for practice. Child Family Community Australia, Paper 45. Melbourne: Australian Institute of Family Studies.

Smith, L. (2005). Building a research agenda for indigenous epistemologies and education. Education Quarterly, 36(1), 93-95. doi.org/10.1525/aeq.2005.36.1.093. 
Snyder, S. (2013). The simple, the complicated, and the complex: educational reform through the lens of complexity theory. OECD ilibrary. Retrieved from http://www.oecd-ilibrary.org/education/the-simple-the-complicated-and-thecomplex-educational-reform-through-the-lens-of-complexitytheory_5k3txnpt1lnr-en?crawler=true.

Song, Y. (2018). Fostering Culturally Responsive Schools: Student Identity Development in Cross-Cultural Classrooms. International Journal of Education \& the Arts, 19(3), 24.

Spillane, J. (2006). Distributed leadership. San Francisco, California: Jossey-Bass.

Spillane, J., Camburn, E., \& Stitziel Pareja, A. (2007). Taking a distributed perspective to the school principal's workday. Leadership and Policy in Schools, 6(1), 103-125. doi.org/10.1080/15700760601091200.

Spillane, J. P., Halverson, R., \& Diamond, J. B. (2001). Investigating school leadership practice: A distributed perspective. Educational Researcher, 30(3), 23-28. Doi.org/10.3102/0013189X030003023.

Spillane, J. P., Halverson, R., \& Diamond, J. (2004). Towards a theory of leadership practice: A distributed perspective. Journal of Curriculum Studies, 36(1), 334. Doi.org/10.1080/ 0022027032000106726.

Spretnak, C., \& Capra, F. (1986). Green politics. Santa Fe, NM: Bear.

Stake, R. (2006). Multiple case study analysis. New York: The Guilford Press.

Starkey, L., Sylvester, A., \& Johnstone, D. (2017). Negotiating digital divides: Perspectives from the New Zealand schooling system. Journal of Research on Technology in Education, 49, 31-42.

Stewart, J. (2006). Transformational Leadership: An Evolving Concept Examined through the Works of Burns, Bass, Avolio, and Leithwood. Canadian Journal of Educational Administration and Policy, 54,1-29.

Stoll, L. (1999). School culture: black hole or fertile garden for school improvement? In J. Prosser (Ed.), School culture (pp. 30-47). London: SAGE Publications Ltd doi: 10.4135/9781446219362.n3

Stone, M., \& Center for Ecoliteracy (2009). Smart by nature: Schooling for sustainability. California: Watershed Media.

Stroh, D. (2015). Systems Thinking for Social Change: A Practical Guide to Solving Complex Problems, Avoiding Unintended Consequences, and Achieving Lasting Results. United States of America: Chelsea Green Publishing. 
Terrell, R., \& Lindsey, B. (2009). Culturally proficient leadership: The personal journey begins within. Thousand Oaks, California: Corwin Press.

Theoharis, G., \& Scanlan, M. (2015). Leadership for increasingly diverse schools. New York: Routledge.

Thrupp, M. (2010). Not so scholarly but certainly on-message: The leadership BES and its significant silences. Journal of Educational Leadership, Policy and Practice, 25(1),4-10.

Thrupp, M. (2015). Poor performers or just plain poor? Assumptions in the neoliberal account of school failure. Waikato Journal of Education, 20th Anniversary Collection, 20(3), 169-181. Retrieved from http://wje.org.nz/index.php/WJE/article/view/232.

Thrupp, M. (2017). The search for better educational standards: A cautionary tale. Switzerland: Springer.

Thrupp, M., \& Willmott, R. (2003). Education management in managerialist times: Beyond the textual apologists. Berhsire, England: Open University Press.

Timperley, H. (2011). Knowledge and the leadership of learning. Leadership and Policy in Schools, 10(2), 145-170. doi.org/10.1080/15700763.2011.557519.

Tollefson, K., \& Magdaleno, K. R. (2016). Educational leaders and the acknowledgement gap. Journal of School Leadership, 26(2), 37-63.

Tomorrow's Schools Independent Taskforce. (2018). Our schooling futures: Stronger together, Whiria Nga Kura Tuatinitini. Wellington: Ministry of Education, New Zealand.

Tooms, A. K., \& Boske, C. (Eds.). (2010). Bridge leadership: Connecting educational leadership and social justice to improve schools. Charlotte, NC: Information Age.

Tuana, N. (2007). Conceptualizing moral literacy. Journal of Educational Administration, 45(4), 364-378. Doi.org/10.1108/09578230710762409.

Tubin, D. (2017). Leadership identity construction practices: The case of successful Israeli school principals. Educational Management Administration \& Leadership, 45(5), 790-805. https://doi.org/10.1177/1741143216682503.

Tyler, T. (2011). In quest of a dropout theory: Examining the utility of an ecological approach through survey research. UNLV theses, dissertations, professional papers, and capstones. Retrieved from https://igitalscholarship.unlv.edu/thesesdissertations/1246. 
University of Manchester (2011). Emerging patterns of school leadership 2: A deeper understanding. Manchester, England: National College for Leadership of Schools and Children's Services.

Uhl-Bien, M., Marion, R., \& McKelvey, B. (2007). Complexity leadership theory: Shifting leadership from the industrial age to the knowledge era. Leadership Institute Faculty Publications. Retrieved from http://digitalcommons.unl.edu/leadershipfacpub/18.

Vygotsky, L. (1979). The genesis of higher mental function. In T.V. Wertsch (Ed.\& Trans). The concept of activity in Soviet psychology. Armonl, NY: M.E. Sharpe Incorporated.

Wafula, E. (2015). Theory and practise of educational leadership. The strategy of involving. Germany: GRIN Verlag.

Waheed, Z., Hussin, S., \& Bin Megat Daud, M. A. K. (2018). The best practices for school transformation: A multiple-case study. Journal of Educational Administration, 56(1). Bingley, UK: Emerald Publishing Limited.

Walker, D., Sorenson, C., Smaldino, S., \& Downey, P. (2008). A model for professional development school intervention: REAL findings. SchoolUniversity Partnerships, 2(1), 6-26.

Walker, K., Kutsyuruba, B., \& Bishop-Yong, N. (2011). The principle of best interests of students in the principalship. Journal of Educational Administration and Foundations, 22(2), 27-60.

Wan, G. (2008). The education of diverse student populations: A global perspective. Athens, Ohio: Springer Science \& Business Media.

Weick, K. E., Sutcliffe, K. M., \& Obstfeld, D. (2005). Organizing and the process of sensemaking. Organization Science, 16(4), 409-421. doi.org/10.1287/orsc.1050.0133

Weinstein, J., Raczynski, D., \& Peña, J. (2018). Relational trust and positional power between school principals and teachers in Chile: A study of primary schools. Educational Management Administration \& Leadership. https://doi.org/10.1177/1741143218792912

Wenmoth, D. (2014). Introducing the 2014 ten trends. New Zealand. Retrieved from http://www.edtalks.org/video/introducing-2014-ten-trends.

Wheatley, M. J. (2006). Leadership and the new science: Discovering order in a chaotic world ( $3^{\text {rd }}$ ed.). San Francisco: Berrett-Koehler. 
Wheatley, M. J., \& Kellner-Rogers, M. (1998). Simpler way. San Francisco: BerrettKoehler.

Wheatley, M., \& Frieze, D. (2011). Walk out walk on: A learning journey into communities daring to live the future now. San Francisco: Berrett-Koehler.

Whitehead, M. (1992). The concepts and principles of equity and health. International Journal of Health Services: Planning, Administration, Evaluation, 22(3), 429-445. doi.org/10.2190/986L-LHQ6-2VTE-YRRN.

Wielkiewicz, R., \& Stelzner, S. (2005). An ecological perspective on leadership theory, research, and practice. Psychology faculty publications. Retrieved from https://digitalcommons.csbsju.edu/psychology_pubs/63.

Wilson, S., Cummings, S., Jackson, B., \& Proctor-Thomson, S. (2017). Revitalising leadership: Putting theory and practice into context. New York, Routledge.

Woods, P. (2005). Democratic leadership in education. London: Routledge. doi.org/10.4135/9781446211885.

Woods, P. A., \& Roberts, A. (2018). Collaborative school leadership in a global society: A critical perspective. Educational Management Administration \& Leadership. doi.org/10.1177/1741143218759088.

Wright, N. (2011). Between 'bastard' and 'wicked' leadership? School leadership and the emerging policies of the UK Coalition Government. Journal of Educational Administration and History, 43(4), 345-362. doi.org/10.1080/00220620.2011.606893.

Wylie, C. (2012). Vital connections: Why we need more than self-managing schools. Wellington: New Zealand Council for Educational Research. Retrieved from http://www.nzcer.org.nz/nzcerpress/vital-connections.

Wylie, C. (2016). Principals and their work: Findings from the NZCER national survey of primary and intermediate schools 2016. Wellington: New Zealand Council for Educational Research Press.

Wylie, C. (2017). Nothing great is easily won. In R. McNae, M. Morrison, \& R. Notman (Eds.), Educational leadership in Aotearoa New Zealand: Issues of context and social justice. (pp. 14-28). Wellington, NZ: NZCER Press.

Wylie, C., McDowall, S., Ferral, H., Felgate, R., \& Visser, H. (2018). Teaching practices, school practices, and principal leadership: The first national picture 2017. Retrieved from http://www.nzcer.org.nz/research/publications/teaching-practices-schoolpractices-and-principal-leadership-first-national. 
Yang, Y. (2014). Principals' transformational leadership in school improvement. The International Journal of Educational Management, 28(3), 279-288. doi.org/10.1108/IJEM-04-2013-0063.

Yin, R. K. (2014). Case study research: Design and methods ( $5^{\text {th }}$ ed.). Los Angeles: Sage Publications Limited.

Yin, R. K. (2018). Case study research and applications: Design and methods $\left(6^{\text {th }}\right.$ ed.). Los Angeles: Sage Publications Limited.

Zaccaro, S. J. (2007). Trait-based perspectives of leadership. American Psychologist, 62(1), 6-16. doi.org/10.1037/0003-066X.62.1.6.

Zepke, N., \& Leach, L. (2010). Beyond hard outcomes: 'soft' outcomes and engagement as student success. Teaching in Higher Education, 15(6), 661673. doi.org/10.1080/13562517.2010.522084. 


\section{Appendices}

Appendix A: A chronology of relevant developments related to the transformation of educational leadership in NZ

Appendix B: The scope of literature used in the review

Appendix C: Principal information sheet

Appendix D: Research schedule - an example

Appendix E: $\quad$ Field notes - an example

Appendix F: Principal semi-structured interview schedule

Appendix G: Various participant semi-structured interview schedule

Appendix H: Coded transcript - an example

Appendix I: Tabular display of coded headings - an example

Appendix J: $\quad$ BOT/Principal letter

Appendix K: BOT/Principal consent forms

Appendix L: Information sheets - staff, community and students

Appendix M: Consent forms - staff, community and students

Appendix N: Formal observations - information and consent forms 


\section{Appendix A: A chronology of relevant NZ developments of educational leadership}

\begin{tabular}{|c|c|c|c|c|}
\hline Year & Title & Description & Author/organisation & Publication type \\
\hline 1988 & $\begin{array}{l}\text { Administering for excellence: } \\
\text { Effective administration in } \\
\text { education }\end{array}$ & $\begin{array}{l}\text { A taskforce headed by Brian Picot, reported on the state of New } \\
\text { Zealand's education system. } \\
\text { The report recommended that all schools become controlled by locally } \\
\text { elected BOT who would be responsible for learning outcomes, } \\
\text { budgeting, and the employment of teachers. Each institution would } \\
\text { produce a charter including a mission statement, MOE prescribed } \\
\text { requirements of safety, equity and national standards. }\end{array}$ & $\begin{array}{l}\text { Department of Education } \\
\text { Taskforce }\end{array}$ & Report \\
\hline 1989 & Tomorrow's Schools & The government's policy response to the above report. & MOE & Policy \\
\hline 1995 & $\begin{array}{l}\text { Targeted Funding for Educational } \\
\text { Achievement }\end{array}$ & $\begin{array}{l}\text { A policy that introduced decile rankings so that funding streams could } \\
\text { better address educational under-performance resulting from socio- } \\
\text { economic disadvantage. }\end{array}$ & MOE & Policy \\
\hline 2007 & New Zealand Curriculum & $\begin{array}{l}\text { Significant review of the NZC was published to reflect greater } \\
\text { coherence, sustainability and place. }\end{array}$ & MOE & Curriculum \\
\hline 2007 & BES series & $\begin{array}{l}\text { A report that synthesised international evidence about what works to } \\
\text { improve education outcomes for diverse students. Each synthesis } \\
\text { focused on either professional learning, quality teaching or leadership. } \\
\text { The series addressed leadership practice in schools and the design of } \\
\text { PLD. }\end{array}$ & MOE & Report \\
\hline 2008 & Kiwi Leadership for Principals & $\begin{array}{l}\text { A position paper that gave a model for leadership and included the } \\
\text { qualities, knowledge, and skills required to lead in NZ schools. }\end{array}$ & MOE & Position paper \\
\hline
\end{tabular}




\begin{tabular}{|c|c|c|c|c|}
\hline Year & Title & Description & Author/organisation & Publication type \\
\hline 2009 & $\begin{array}{l}\text { School leadership and student } \\
\text { outcomes: Identifying what works } \\
\text { and why, best evidence synthesis }\end{array}$ & $\begin{array}{l}\text { A report on effective leadership practices that provided support for } \\
\text { leaders by describing the characteristics of leadership that most } \\
\text { improved outcomes for student. }\end{array}$ & Robinson et al., (2009) & Report \\
\hline 2010 & National Standards & $\begin{array}{l}\text { This policy outlined expectations for students in reading, writing and } \\
\text { mathematics throughout their primary school career. }\end{array}$ & MOE & Policy \\
\hline 2010 & Tu Rangatira & An exploration of Māori Medium Educational Leadership in NZ. & MOE & Report \\
\hline 2010 & He Kākano & $\begin{array}{l}\text { A strategic school-based professional development programme with an } \\
\text { explicit focus on improving culturally responsive leadership to ensure } \\
\text { success for Maori. }\end{array}$ & MOE & Strategy \\
\hline 2013 & $\begin{array}{l}\text { Ka Hikitia: accelerating success } \\
\text { 2013-2017 }\end{array}$ & $\begin{array}{l}\text { A strategy aimed towards rapid change, so that all Māori students gain } \\
\text { the skills, qualifications and knowledge they need to enjoy and achieve } \\
\text { education success as Māori. }\end{array}$ & MOE & Strategy \\
\hline 2013 & $\begin{array}{l}\text { Pasifika Education Plan 2013- } \\
2017\end{array}$ & $\begin{array}{l}\text { A strategy to ensure all Pasifika students gain the skills, qualifications } \\
\text { and knowledge they need to enjoy and achieve education success. }\end{array}$ & $\begin{array}{l}\text { Parata, Joyce, Clark, \& } \\
\text { Benny }\end{array}$ & Strategy \\
\hline 2015 & Investing in Education Success & $\begin{array}{l}\text { A policy to lift the performance of all students, especially those at risk } \\
\text { of underachievement, with a focus on building teaching capacity. }\end{array}$ & MOE & Policy \\
\hline 2016 & Evaluation Indicators & $\begin{array}{l}\text { A new version (first published in 2003) of indicators that explored the } \\
\text { role of evaluation in the process of improvement to ensure schools were } \\
\text { accountable for their stewardship. }\end{array}$ & ERO & Indicators \\
\hline 2016 & Principal Professional Standards & $\begin{array}{l}\text { Provided a baseline for a principal's performance agreement, which } \\
\text { reflected the school or board goals, the principal's job description, more } \\
\text { specific objectives, and the New Zealand Education Council's criteria } \\
\text { for registration as a teacher. }\end{array}$ & $\begin{array}{l}\text { Teaching Council of } \\
\text { Aotearoa, New Zealand }\end{array}$ & Performance baseline \\
\hline 2017 & $\begin{array}{l}\text { Code of Professional } \\
\text { Responsibilities and Standards for } \\
\text { the Teaching Profession }\end{array}$ & $\begin{array}{l}\text { An outline of the conduct expected of all teachers, leaders and teaching } \\
\text { experts to articulate the expectations and aspirations of the profession. }\end{array}$ & $\begin{array}{l}\text { Teaching Council of } \\
\text { Aotearoa, New Zealand }\end{array}$ & Code of conduct \\
\hline
\end{tabular}




\begin{tabular}{|c|c|c|c|c|}
\hline Year & Title & Description & Author/organisation & Publication type \\
\hline 2017 & Emerging Leaders & $\begin{array}{l}\text { A pilot programme for emerging leaders in schools due to be completed } \\
\text { at the end of } 2019 \text {. }\end{array}$ & MOE & Pilot programme \\
\hline 2017 & $\begin{array}{l}\text { Principals and their work: findings } \\
\text { from the NZCER national survey } \\
\text { of primary and intermediate } \\
\text { schools } 2016\end{array}$ & $\begin{array}{l}\text { Principals in primary and intermediate schools took part in a national } \\
\text { report that asked about morale, leadership development, professional } \\
\text { learning, achievements, challenges, and career path. }\end{array}$ & Cathy Wylie & Report \\
\hline 2018 & Living Standards Framework & $\begin{array}{l}\text { A framework devised to help successive governments understand how } \\
\text { future policy choices will affect a New Zealanders' living standard over } \\
\text { time. }\end{array}$ & Treasury NZ & Framework \\
\hline 2018 & Tomorrows Schools Review & $\begin{array}{l}\text { A task force headed by Bali Haque to review the way schools have been } \\
\text { governed, managed and administered since the implementation of the } \\
\text { Tomorrow's Schools policy of } 1989 \text {. }\end{array}$ & $\begin{array}{l}\text { Tomorrow's } \quad \text { Schools } \\
\text { Independent Taskforce }\end{array}$ & Report \\
\hline 2018 & $\begin{array}{l}\text { Leadership Strategy for the } \\
\text { Teaching Profession }\end{array}$ & $\begin{array}{l}\text { A system-level approach to developing leadership capability for } \\
\text { teaching professionals. }\end{array}$ & $\begin{array}{l}\text { Education Council of } \\
\text { Aotearoa, New Zealand }\end{array}$ & Strategy \\
\hline 2018 & $\begin{array}{l}\text { Teaching practices, school } \\
\text { practices and principal leadership: } \\
\text { the first national picture } 2017\end{array}$ & $\begin{array}{l}\text { This report was commissioned by the Ministry of Education to provide: } \\
\text { school-level data that can be used by schools to review and plan to } \\
\text { improve teaching and leadership capability. At a national level the data } \\
\text { would be useful for evaluating the impact of policy change. }\end{array}$ & $\begin{array}{l}\text { Education Council of } \\
\text { Aotearoa, New Zealand }\end{array}$ & Report \\
\hline 2018 & Leadership Capability Framework & $\begin{array}{l}\text { A framework that intended to provide high-level guidelines for } \\
\text { leadership development based on shared understandings of what } \\
\text { leadership in different contexts looks like in practice. The capabilities } \\
\text { can be used to shape and critically reflect on programmes, practice } \\
\text { inquires, organisational strengths and inform decision about PD } \\
\text { requirements. }\end{array}$ & NZCER & Framework \\
\hline
\end{tabular}




\section{Appendix B:}

\section{The scope of literature used in the review}

\begin{tabular}{|c|c|c|c|}
\hline Leadership theory/approach & Basic description & Main characteristics & Researchers scoped \\
\hline Adaptive/Strategic & $\begin{array}{l}\text { Structure for helping individuals } \\
\text { and organisation thrive in } \\
\text { changing environments }\end{array}$ & $\begin{array}{ll}- & \text { Organisational justice } \\
- & \text { Emotional intelligence } \\
- & \text { Learning focused } \\
- & \text { Capacity building } \\
- & \text { Sustainable }\end{array}$ & Heifetz et al. (2009) \\
\hline As-practice (leaderful) & $\begin{array}{l}\text { Leadership as practice, rather than } \\
\text { evident in traits or behaviours of } \\
\text { individuals }\end{array}$ & $\begin{array}{l}\text { - } \quad \text { Creating awareness of the complex challenges } \\
\text { - } \quad \text { Facing up to the consequences of inaction } \\
\text { - } \text { pupporting cross-organisation teams to find new } \\
\text { - Creating the social and cultural conditions for positive } \\
\text { use of power } \\
\text { Making sure that processes are aligned and fit for } \\
\text { purpose }\end{array}$ & $\begin{array}{l}\text { Dalcher (2018) } \\
\text { Lambert (2003a) } \\
\text { Ospina \& Sorenson, } 2006 \\
\text { Raelin (2014) } \\
\text { Raelin (2017) }\end{array}$ \\
\hline Bridge & $\begin{array}{l}\text { Explores intersection between } \\
\text { social justice and educational } \\
\text { leadership }\end{array}$ & $\begin{array}{l}\text { Focused on outcomes of equity, opportunity and } \\
\text { productivity } \\
\text { - Traverse spaces between people }\end{array}$ & Tooms and Boske (2010) \\
\hline Change & $\begin{array}{l}\text { Describes what leaders must do to } \\
\text { initiate change }\end{array}$ & $\begin{array}{ll}- & \text { Clear focus } \\
- & \text { Develop capacity in others } \\
- & \text { Earn trust } \\
- & \text { Understand your influence } \\
- & \text { Comprehend the complex } \\
- & \text { Remain hopeful }\end{array}$ & Fullan (2011) \\
\hline Constructivist & $\begin{array}{l}\text { Engages reciprocal learning in } \\
\text { community towards shared goals }\end{array}$ & $\begin{array}{ll}- & \text { Improves learning capacity of school } \\
- & \text { Focused on reciprocal and purposeful learning } \\
- & \text { Encourages growth of human potential } \\
- & \text { Adult and student learning interlinked } \\
\text { - } & \text { Diversity provides complexity }\end{array}$ & Lambert et al. (2002) \\
\hline
\end{tabular}


Leadership theory/approach

Culturally Responsive

Generative

Instructional/Pedagogical/

Leadership for Learning

\section{Basic description}

A form of leadership critical in

working with marginalised group

to address barriers to achievement

Stimulates new ideas

Core focus on curriculum,

teaching pedagogy and student

achievement exhibit an ethic of care

develop cultural awareness in the school and

community

demonstrate and promote inclusive practice

challenge deficit theorising, inequitable principles and practises

Focused on action and growth

Challenges assumptions and fosters inquiry

Thinks outside the problem to find alternative actions

Encompasses learning and evolution

\section{Guiding and supporting}

Driven by strong moral purpose

Improves capabilities of school

Being a learner

\section{Moral /Servant/Social Justice}

Moral/Servant/Social Justice

Motion
Leaders guided by dignity and rights of others
Leaders who make conscious choice to serve others Develop capacity in others

Problem based

Value driven decision-making

Enriches individuals for greater collective gains

Movement towards positive progress

\section{Understand nature of change}

Collaborative

Learning as both an individual and organisational task

Driven by integrity and competence

Developing capacity in others

Results focused

\section{Researchers scoped}

Terrel \& Lindsey (2009)

Klimek, Ritzenhein \& Sullivan (2008)

Bush (2010)

Dufour \& Marzano (2011)

Glickman, Gordon \& Ross-Gordon (2001)

Marzano, Walters \& McNulty (2005)

Robinson et al. (2008)

Robinson et al. (2009)

Robertson \&Timperley (2011)

Stoll, Fink \& Earl (2003)

Woods \& Roberts (2018)

Blanchard and Broadwell (2018)

Branson \& Gross (2014)

Crippen (2004)

Kramer, Enomoto \& Enomoto (2014)

McNae, Morrison \& Notman (2017)

Portelli \& Campbell-Stephens (2009)

Sergiovanni (1996)

Sergiovanni (2003)

Fullan (2009) 
Leadership theory/approach

Place-based

Relational

Postheoric

Shared/Collaborative/Distributed/Democratic

\section{Social Justice}

\section{Basic description}

Endeavouring to improve the quality of life for communities living in a specific place

Individual and organisational practice that progresses both social wellbeing and learning

Resides in the spaces between leaders and followers

Shared contribution of leader and/or follower in a way that draws on strengths and/or brings together formal and informal leaders

Improves teacher instruction at a deep level

\section{Main characteristic}

Community as learning environmen

Connection and relevance

Partnerships and permeability

Inquiry and design

Student centred

Interdisciplinary

Building relationships

Emancipation for better operation and specialisation

Focused on growth and development

\section{Focus on being in leadership}

Building high quality unique partnerships between people

Efficiency of the wider collective important

Focuses on practice-based wisdom

Shared vision for coherent programme

Inquiry-based decisions and practice

Reflective practice that leads to innovation

Shared purpose and decision-making

Develops learning culture

A philosophy of co-developed values

Develops deep relationships

Develops both self-efficacy and collective efficacy
Giles \& Palmer (2015)

Dalcher (2018)

Jackson (2017)

Researchers scoped

Bryk et al. (2015)

Elmore (1979)

Elmore (2000)

Fullan (2008)

Gronn (2003)

Gronn, (2009)

Harris (2009)

Lambert (2002a)

Lambert (2002b)

Lambert (2003a)

Lambert (2003b)

Barrera-Osorio et al. (2009)

Woods (2005)

Wheatley (2006)

Wielkiewicz \& Stelzner (2005)

Woods \& Roberts (2018)

Advocate and be critical

Increase awareness of oppression

Bishop (2008)

Bogotch \& Shields (2014)

Mcnae Morrison \& Notman (2017) 


\section{Leadership theory/approach}

Sustainable

\section{Systemic}

Identifies need for collective change through an inspiring vision

\section{Transformationa}

Transformative
Leader and follower exchange

services for reward and control.

Also, role-based

\section{Basic description}

Collective goals for inspiring others and sustaining ongoing change

Describes a system thinking approach to problem-solving

Deconstruct socio-cultural

frameworks to effect deep and equitable change that works towards transformation for both individual and collective purpose

\section{Main characteristic}

Change focused - both emergent and designed Systems thinking

Sustainability mind-set

Socially responsible

Share data and focus on results

Able to work with and connected to various types of

groups

Future-focused vision and purpose

Thinking locally and globally

Build relationships and trust

Empowering collective effective communication

Strategic thinkers

Leading change across system - not just within own school

- $\quad$ Co-create support

Leader directs needed change

Pre-destined goals

Emphasis on individual good

\section{Shared vision with followers}

Loyal and motivated followers

Collaborative culture

Fosters and develops practice

Solves problems efficiently

Leader seeks to change existing systems and practices

Willing to take risks to stimulate change

Leaders are charismatic and

inspirational

Leaders motivate followers to achieve more than organically planned

Respectful and motivational climate

Staff are valued and supported

Work for common organisational good
Hargreaves (2007)

Hargreaves \& Fink (2005)

Fullan (2001)

Heck \& Weiss (2005)

James Irving Foundation (2017)

Senge (2006)

Walker et al., (2008)

Hiller et al., (2006)

Hohepa \&Lloyd (2008)

MacNeill et al. (2018)

Wafula (2015)

Leithwood et al. (2000)

Leithwood et al (2009)

Moshayedi (2018)

Shields (2013) 
Leadership theory/approach

Translational
Basic description

Apply context to generic

standards-based practice

\section{Main characteristic}

Fosters a new way of imagining the world by

challenging status quo and levelling the playing fields

Builds inclusive vision

Moral courage

Creates an environment where recognition, respect and

regard for individuals is important

School as a private and public good

Exploring beliefs, values, assumptions and power

Focus on interconnectedness, interdependence and

global awareness
Researchers scoped

Shoho et al. (2010) 


\section{Appendix C:}

\section{Principal information sheet}

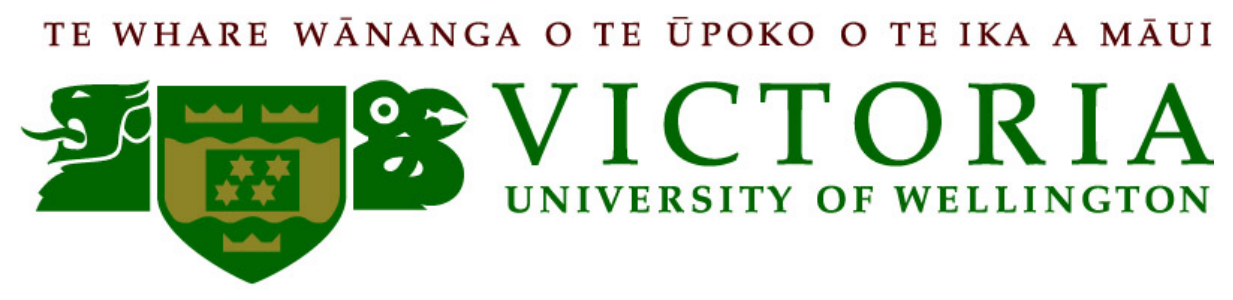

$\underline{\text { Research project - principal information sheet }}$

"An investigation into leadership practice in low socio-economic New Zealand primary schools: How principal leaders engage for educational success."

\section{Researcher}

I am Angela Millar, a PhD student at the Faculty of Education, Victoria University of Wellington, New Zealand. As part of my doctoral study, I am conducting research to examine the role of principal leadership practices in promoting educational outcomes in low socio- economic primary school settings. This research has ethical approval from the Victoria University College of Education Ethics Committee.

This sheet gives you information about this study. If you have any questions or would like to receive further information about the project, please contact me at xxxx. You can also contact my supervisor Kate Thornton at xxxx, and Liz Jones at xxx.

The research has been approved by the Faculty of Education Human Ethics Subcommittee under delegated authority from the Victoria University Human Ethics Committee. If you have any ethical questions about this research please contact Dr Allison Kirkman, Chair of the Human Ethics Committee, Victoria University of Wellington on $\mathrm{xxx}$.

\section{The purpose of my PhD research}

This research aims to contribute to the knowledge base of how principal leadership practice impacts on educational success within the social complexities of a low socio economic schooling community. 


\section{Research questions}

"How do principal leaders practise leadership in low socio economic primary school contexts for educational success?"

- What do the stakeholders in the LSE context perceive as educational success?

- What leadership practice patterns are in evidence in the daily life of a principal?

- In what ways are principal practice influencing of or influenced by context?

- What beliefs, values and ideas underpin principal practice patterns in the LSE context for educational success?

\section{Research process}

My study will be conducted in three primary schools across New Zealand. The school and you, the principal, have been chosen because together you meet the criteria for participating in research aiming to develop an understanding of the phenomenon of effective principal leadership in a low socio economic schooling context. To achieve an in-depth understanding, I plan to include a variety of data gathering methods in the research process. The attached 'Proposed Research Schedule' gives you an idea of what this might look like over the course of a school term.

Initially, I would like to interview you to begin to understand who you are as leader, to shadow you in order to time diary a day in your life as the principal of X school. The interview and observation will help develop a fuller understanding of the complexity of a principal's practices and context as well as identifying valued educational success. Additionally I would like to observe at least two activities where you are actively engaged in your work and these would be videoed. I would not be evaluating the participation of any of the staff or community but rather taking note of how you adapt your practices according to context. Group interviews with staff, community and students would also be useful to ascertain alternative perceptions of your practice, contextual needs and educational success.

By observing and shadowing you, interviewing you and your school community and gathering documentation over a term, I will be able to address the research questions as well as describe the phenomenon of effective principal leadership from within your unique school context. I may return to the school once or twice the following term for any necessary follow up.

\section{Interviews, observations \& documentation}

Our first meeting would be an in-depth interview lasting approximately an hour. The purpose of this interview is to elicit a response to beliefs and values that underpin your practices. I will be especially interested in hearing your personal leadership story.

You may elect at least two formal observations. The focus of these observations is to observe the active participation of your school community. This may mean observing a staff meeting or a BOT meeting for example. During these observations I would record all responses via video or, if preferred, an audio device. Data generated from the formal observations will form the basis of further discussions with you. Participants who make responses in the formal observations will not have these responses attributed back to them. 
Shadowing you for a day would occur for the sole purpose of attaining an accurate sample of your day. This involves noting the theme of each activity you are involved in and with whom. No other notes will be taken at this time. By having the researcher do this, a clearer understanding about how a principal spends their day will be noted and whom you connect with. However, if the preferred, you may elect to do your own time diary.

The student group interview will be in the form of an in-class activity, lasting up to one period for those students in the class whose parents have given permission for them to be involved and whose teacher shows a keen interest. Any identifying features will be removed. This activity, designed in draft, is most appropriate to senior students (year 5-8). Students will be asked to design an advertisement for a new school principal. The draft activity will be finalized by the researcher, the teacher involved and you the principal and resubmitted to the Victoria University ethics committee for final approval.

Off site, I will be reflecting on my experiences at the school via my research journal. Here I will be making notes of the climate of the school, how my interactions have gone and what I could be doing differently or better as a researcher. I am happy to have these field notes available for you to see.

\section{Research participants}

I am asking for you as the principal to volunteer to take part in this research from within your school context. Additionally this is an opportunity to ensure all of those from within the school context are given the opportunity to represent their understandings of how principal leadership practises support educational success and thus should include volunteer students, staff and community members.

\section{Confidentiality and access to information}

The information provided is strictly confidential. Your school and yourself will not be identifiable. Information collected will be kept in a locked filing cabinet at the office of Angela Millar, Victoria University of Wellington.

You will be provided with feedback through ongoing discussions both during and after the data gathering process. As well as this summary reports on the formal observation/s and full transcripts of all interviews can be expected. You will be given the opportunity to change or delete your comments if you wish.

\section{Participation}

You may withdraw from the research project for any reason at any stage prior to the data analysis and any data provided would then be destroyed.

\section{The participation for the principal would involve:}

- $\quad$ Being available for interviews, shadowing, observations and any required follow up discussions.

- $\quad$ Be available to read summary reports and offer feedback as required.

- Agreeing that any pertinent school documentation such as the school charter or ERO report be made available for the researcher Angela Millar. 
- $\quad$ Agreeing that the information given can be used by the researcher, Angela Millar for this study.

\section{Publication of results}

The information gained in this research will be used for publication purposes including academic or professional journals, conference papers and appropriate articles and for the deposit of the thesis at the Victoria University of Wellington library.

Your consent for this study is highly valued.

Thank you

Angela Millar 


\section{Appendix D:}

\section{Research schedule - an example}

TE WHARE WĀNANGA O TE ŪPOKO O TE IKA A MĀUI

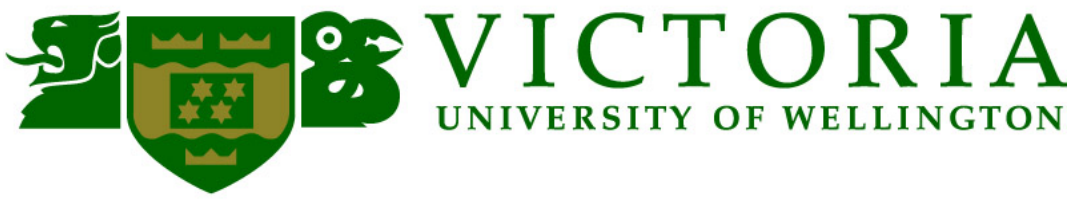

\begin{tabular}{|c|c|c|c|c|c|c|c|c|}
\hline Week & $\begin{array}{c}\text { Interview } \\
- \\
\text { principal }\end{array}$ & $\begin{array}{c}\text { Shadowing } \\
- \\
\text { observation }\end{array}$ & $\begin{array}{c}\text { Focus } \\
\text { groups - } \\
\text { staff \& } \\
\text { communit } \\
y\end{array}$ & $\begin{array}{l}\text { Student- } \\
\text { in-class } \\
\text { activity }\end{array}$ & $\begin{array}{c}\text { Formal } \\
\text { Observation/s }\end{array}$ & $\begin{array}{l}\text { Follow- } \\
\text { up } \\
\text { meetings }\end{array}$ & $\begin{array}{c}\text { School } \\
\text { Documentation }\end{array}$ & $\begin{array}{l}\text { Follow-ups } \\
\text { as required }\end{array}$ \\
\hline 1 & $\mathbf{X}$ & & & & & & & \\
\hline 2 & & $\mathbf{X}$ & & & & $\mathbf{X}$ & & \\
\hline 3 & & & & & & & & \\
\hline 4 & & & $\begin{array}{l}\mathbf{X} \\
\mathbf{X}\end{array}$ & & & $\mathbf{X}$ & & \\
\hline 5 & & & & & $\mathbf{X}$ & & & \\
\hline 6 & & & & & & $\mathbf{X}$ & & \\
\hline 7 & & & & & $\mathbf{X}$ & & & \\
\hline 8 & & & & $\mathbf{X}$ & & $\mathbf{X}$ & & \\
\hline 9 & & & & & $\mathbf{X}$ & & $\mathbf{X}$ & \\
\hline 10 & & & & & & $\mathbf{X}$ & & \\
\hline 11 & & & & & & & $\mathbf{X}$ & \\
\hline $\begin{array}{c}\text { Following } \\
\text { Term }\end{array}$ & & & & & & & & $\mathbf{X}$ \\
\hline
\end{tabular}


Appendix E: Researcher field notes - example

\section{Date: Nos $y^{\text {m }}$ cosing}

$\rightarrow$ bivoloment in pre-sclord classes disiciosed. Joder $x$ glitte nore defermine bout ischas taking on. Pre-selat disses $1-2$ wek.

$\rightarrow$ Trying to bal ance engaging ware of

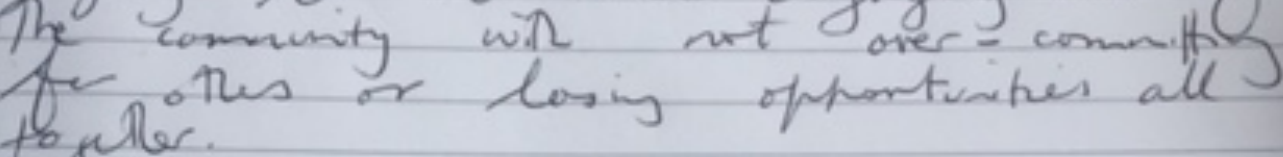
to giler.

$\rightarrow x$ wanly froblem-solung the dilemia it seens. Not divag an exy balace

Cinceluced to priest e lowal Holice offiw polar. Saw 102 parents

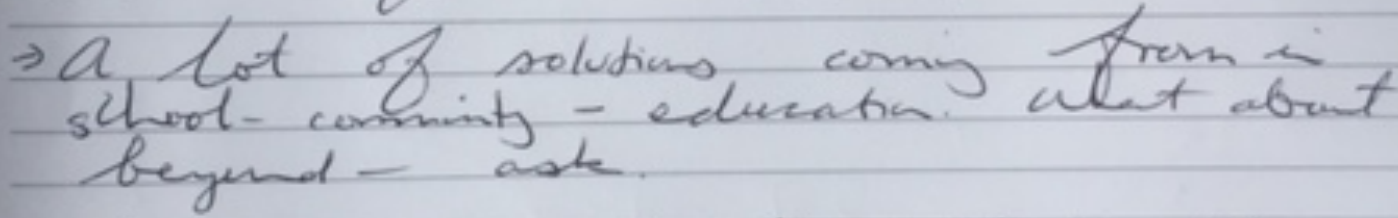
$\rightarrow$ Cvergeng vey frielly Mrd like

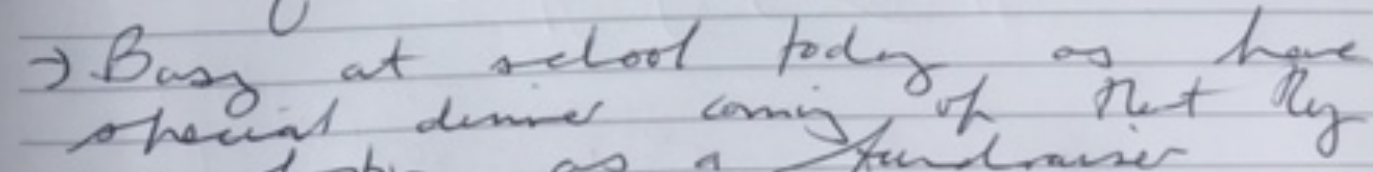
we hasty as a fouldinir

$\rightarrow x$ apheas to have yist so much

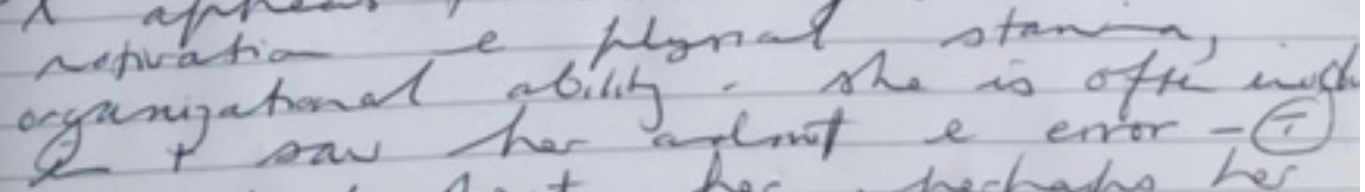

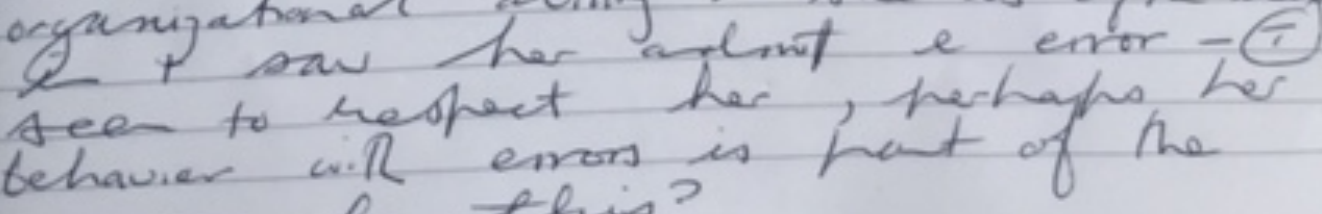
measur for telis? 


\section{Appendix F: Principal semi-structured interview schedule}

\begin{tabular}{|c|c|}
\hline Questions & Principal: in depth interviews... \\
\hline $\begin{array}{l}\text { What beliefs, values and ideas } \\
\text { underpin principal practice patterns } \\
\text { in the LSE context for educational } \\
\text { success? }\end{array}$ & 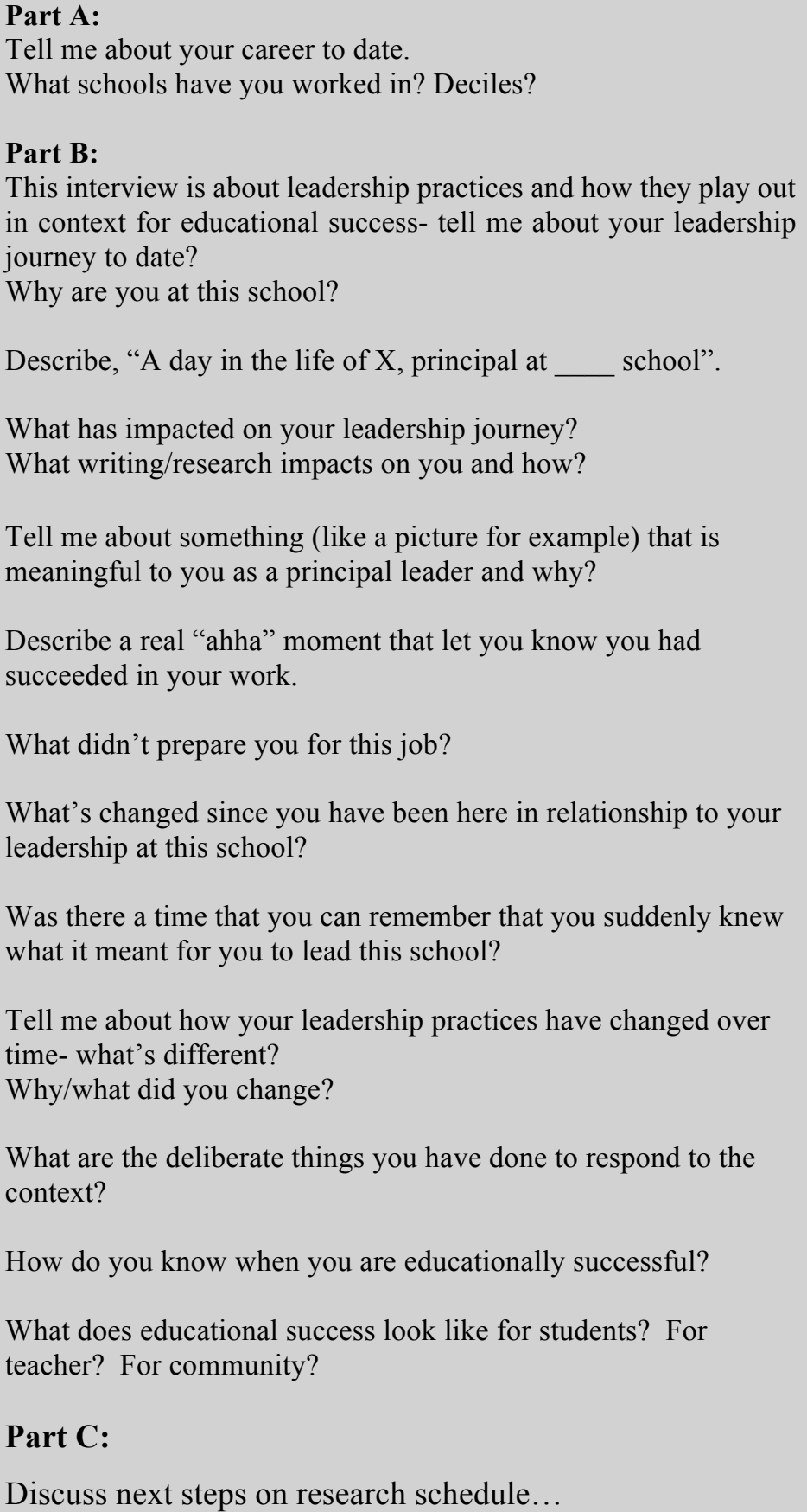 \\
\hline
\end{tabular}


Appendix G: Various participant semi-structured interview schedule

Introduction
Uncommon commonalities
Purpose of session, administration details for group
Check recording devices, group guidelines.
Questions: Themes to identify, deepen and discover
PRACTICES IN CONTEXT:
Tell me about your school?
What is the principal's role in the school?
PRACTICES:
What does this principal do well and how do you know?
EDUCATIONAL SUCCESS:
Define educational success - what is most important and why?
What is the principal's role in creating this?

\section{Focus group guidelines:}

- clarify purpose of discussion

- create hospitable space

- explore questions that matter

- encourage everyone to contribute

- connect diverse perspectives

- listen for insights and share discoveries. 


\section{Appendix H: Coded transcript - an example}

\section{Positive learning climate}

I agree with that because it's not pedagogy that's done to the children it's pedagogy that's, that kind of works with them somehow, to let them be the best they can be but without denying themselves as themselves, we're not trying to make them in to something else.

Q Yeah.

Encouraging positive identity

J A whole, you know a whole bunch of little white kids or something.

$\mathrm{K} \quad$ And there's an urgency with those high expectations.

\section{Expectations}

T Yes.

$\mathrm{K} \quad$ And that's something that l've probably learned from $\mathrm{X}$ I would say because you know over the years I have been here l've known about you know about expecting the best but I haven't been, I haven't had the cattle prod really and l've needed, and I need the cattle prod. I guess probably for me I came here and this is 25 years ago because of the way the school was organised, it was organised in whanau and I had a real need to belong and still very, very much belong and that's because that way of organising the school's been sustained and maintained and so I've got the dual thing going on, I really feel I belong here because the amount of time but also I can so back up the challenge I'm still very, very challenged, I'm always, have always got puzzles of practice and so I guess yeah it's, I'm on a waka that I really want to be on and the best thing for me is that I really want to be on this waka but I so don't want to be leading it so you, yeah I want to be, I'm paddling away there, I'm coming but yeah you lead that, you take that responsibility and.

C Waka doesn't go anywhere without paddlers though.

K Yeah I know I know but that's true.

Teamwork

Developing pedagogy

Q That's true.

$\mathrm{K} \quad$ But yeah just, just I like the direction of the waka, sometimes I think the waka is going too far too fast and that's, that's yeah probably something that I struggle with a little bit. But also when I say belong I feel I belong to my whanau but also belong in my, I feel I belong in my level team and I belong, I have a sense of belonging in the whole school, school.

Q You're all agreeing so that sense of belonging.

Belonging

Tension: speed of change

\section{Teamwork}

Is a real.

$\mathrm{K} \quad$ So when $\mathrm{X}$ says we're great I feel I'm part of really great team and I really, and there's, I love the our nature of this school you know so you know we're sitting down at technology and X's showing me how to do stuff that I can't do and you know there's, there's always someone that's prepared to share their talents, and also share the kids you know with our kids are very much shared so although they are end up being our responsibility there's still a sense of our, our. 
Appendix I: Tabular display of coded headings - an example

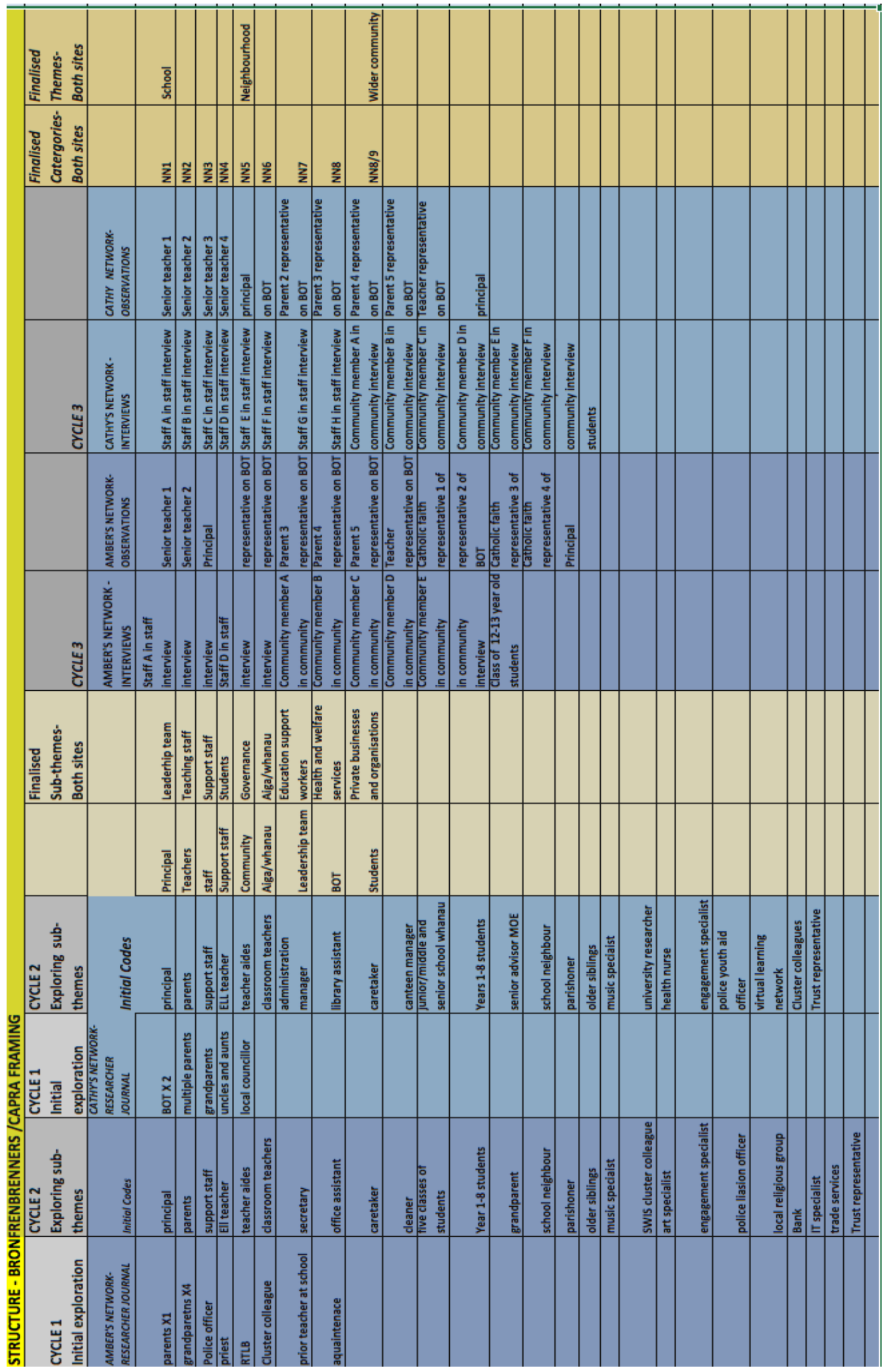




\section{Appendix J:}

\section{BOT/principal letter}

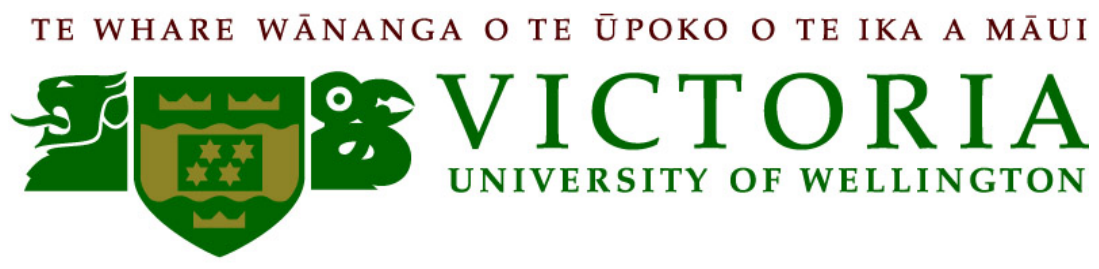

To the Board of Trustees of XXXXXXX School and XXXXX,

In Term X, I am hoping to conduct research at your school entitled, "“'Effective leadership practice in low socio-economic New Zealand primary schools: how principal leaders engage for educational success." The main aim of the research is to contribute to the knowledge base of how principal leadership practice impact on educational success within the social complexities of a low socio economic schooling community.

Since I am asking primarily for the school principal to volunteer to take part in this research from within your school context, I felt it important to gauge their initial interest first. I have indeed discussed this with XXXX your principal, who has expressed a willingness to be involved, both in support of her own professional development as well as the bigger picture research aims and objectives which can be found on the attached information sheet.

What follows, is both an information sheet and a consent form which spells out the research in detail and the requirements of the principal, the school and the schooling community, as well as my own commitments and responsibilities as a researcher. The consent form once signed, is a formal agreement for the research to take place.

I am willing to be contacted or come in to meet with you regarding questions or concerns should the need arise. Please do not hesitate to contact me at the above phone number.

I look forward to working with you in the future.

Kind regards,

Angela Millar

PhD Student, Victoria University of Wellington

Phone: $X X X X X X X$ 


\section{Appendix K:}

\section{BOT/principal consent forms}

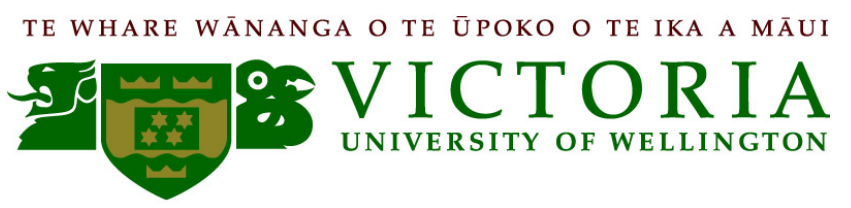

\section{$\underline{\text { Research project - Board of Trustees consent form }}$}

"An investigation into leadership practice in low socio-economic New Zealand primary schools: How principal leaders engage for educational success."

\section{Please read the following:}

$>$ We agree that the principal will be interviewed by the researcher Angela Millar

$>$ We agree that staff who volunteer may be interviewed by the researcher, Angela Millar

$>$ We agree that community who volunteer may be interviewed by the researcher, Angela Millar

We understand that interviews will be recorded and transcribed

We are willing for the researcher to shadow the principal to observe their activities

We are willing for the researcher to observe, record and video up to two activities where the principal is actively involved

We understand that participation by school staff and community is entirely voluntary

We agree that students who volunteer may be involved in an in-class activity and the researcher Angela Millar will be present

We understand that the research is confidential

We understand that the analysis and aggregation of the data obtained may be used for a thesis, for conference papers and/or publication

We understand that participants can withdraw from the research project for any reason at any stage prior to the data analysis and that any data provided would then be destroyed 
We understand that participants will have a chance to check the accuracy of any interviews

We understand that transcripts and tapes and raw data will be destroyed five years after the conclusion of the project.

\section{Please indicate your preference for involvement in the research (tick as} appropriate):

- We have had the project explained to us and have had the chance to ask any questions. We do not give consent for the school to take part in the research

- We would like further questions to be answered about this project.

- We have had the project explained to us and have had the chance to ask any questions. We agree to the school taking part in the above research.

Name School

Signature Date

\section{BOT Request for research summary}

I wish to be given a summary of the research findings:
o Yes
o No

Name:

This research has ethical approval from the Victoria University College of Education. 


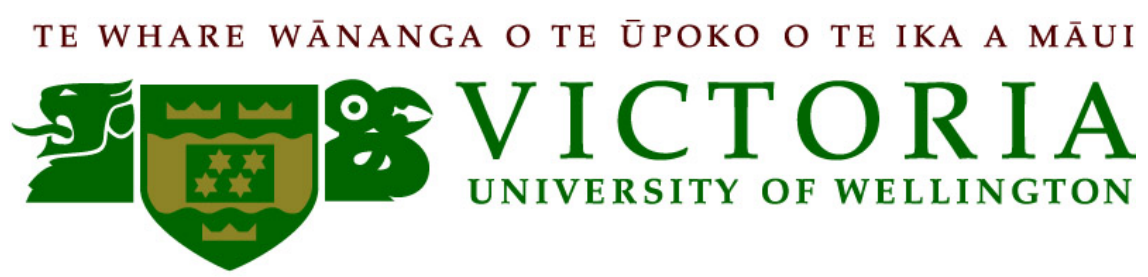

\section{$\underline{\text { Research project- Principal consent form }}$}

"An investigation into leadership practice in low socio-economic New Zealand primary schools: How principal leaders engage for educational success."

\section{Please read the following:}

I agree to be interviewed, shadowed and observed by the researcher Angela Millar

$>$ I understand that interviews will be recorded and transcribed.

$>$ I am willing for the researcher to observe, record and/or video up to two activities where I am actively involved.

I understand that my participation is entirely voluntary.

$>$ I understand that the research is confidential.

$>$ I understand that the analysis and aggregation of the data obtained may be used for a thesis, for conference papers and/or publication.

$>$ I understand that the data collected will only be used for the research project as described in the information sheet.

$>$ I understand that I can withdraw from the research project for any reason at any stage prior to the data analysis and that any data provided would then be destroyed.

$>$ I understand that I will have a chance to check the accuracy of any interviews and observations.

$>$ I understand that transcripts and tapes and raw data will be destroyed five years after the conclusion of the project. 
Please indicate your preference for involvement in the research (tick as appropriate):

- We have had the project explained to us and have had the chance to ask any questions. We do not give consent for the school to take part in the research

- We would like further questions to be answered about this project.

- We have had the project explained to us and have had the chance to ask any questions. We agree to the school taking part in the above research.

Name School

Signature Date

\section{Principal Request for Research Summary}

I wish to be given a summary of the research findings (please tick as appropriate):

$\begin{array}{ll}\text { o } & \text { Yes } \\ \text { o } & \text { No }\end{array}$

Name:

This research has ethical approval from the Victoria University College of Education Ethics Committee. 


\title{
Appendix L:
}

\section{Information sheets - staff, community and students}

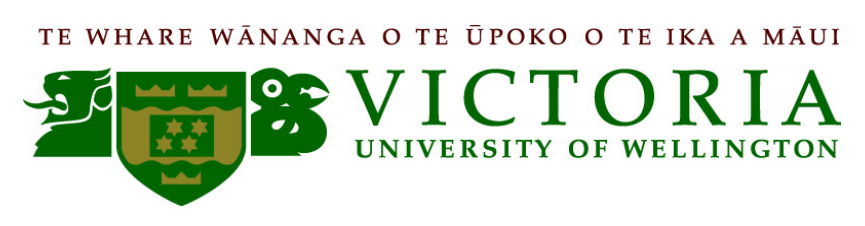

\section{$\underline{\text { Research project, staff information sheet }}$}

\begin{abstract}
"An investigation into leadership practice in low socio-economic New Zealand primary schools: How principal leaders engage for educational success."
\end{abstract}

\section{Researcher}

I am Angela Millar, a PhD student at the Faculty of Education, Victoria University of Wellington, New Zealand. As part of my doctoral study, I am conducting research to examine the role of principal leadership practices in promoting educational outcomes in low socio- economic primary school settings. This research has ethical approval from the Victoria University College of Education Ethics Committee.

This sheet gives you information about this study. If you have any questions or would like to receive further information about the project, please contact me at xxxx. You can also contact my supervisor Kate Thornton at Xxxx, and Liz Jones at xxx.

The research has been approved by the Faculty of Education Human Ethics Subcommittee under delegated authority from the Victoria University Human Ethics Committee. If you have any ethical questions about this research please contact Dr Allison Kirkman, Chair of the Human Ethics Committee, Victoria University of Wellington on $\mathrm{xxx}$.

\section{The purpose of my $\mathrm{PhD}$ research}

This research aims to contribute to the knowledge base of how principal leadership practices impact on educational success within the social complexities of a low socio economic schooling community.

\section{Research question/s}

"In what ways do principals practice leadership in low socio economic primary school contexts to enhance educational success?"

- What do the stakeholders in the LSE context perceive as educational success? 
- What leadership practice patterns are in evidence in the daily life of a principal?

- What ways are principal practices influenced by or influencing of their context?

- What beliefs and values underpin principal's engagement in their context?

\section{Research process}

My study will be conducted in three primary schools across New Zealand. The school and your principal have been chosen because together they meet the criteria for participating in research aiming to develop an understanding of the phenomenon of effective principal leadership in a low socio economic schooling context. To achieve an in-depth understanding, I plan to include several data gathering methods in the research process.

By observing and shadowing the principal in action, interviewing the principal and school community and gathering documentation over a term, I will be able to address the research questions as well as describe the phenomenon of effective principal leadership from within your unique school context. I may return to the school once or twice the following term for any necessary follow up.

\section{Research participants}

I am asking for the principal and volunteers from the school's community to take part in this research. This is an opportunity to ensure all those interested, are given the opportunity to represent their understandings of how principal leadership practices support educational success.

\section{Participation}

Participants may withdraw from the research project for any reason at any stage prior to the data analysis and any data provided would then be destroyed.

\section{The group interview, in-class activity $\&$ the researcher journal}

After gaining permission from the BOT of X School and the principal to undertake the research, I will conduct a staff focus group session, which I would value your input on. This activity will be done during my data collection from XXX to XXX in school. Group interviews lasting a maximum of an hour and a half will be held with staff volunteers. These will be audio recorded. This group will discuss such things as their concepts of educational success as well as the principal's role in creating this.

You may also choose to elect your class for an in-class activity lasting up to one period. Only students whose parents have given permission for them to be involved will take part. Any identifying features will be removed. The activity, designed in draft, is most appropriate to senior students. Students will be asked to design an advertisement for a new school principal. The draft activity will be finalized by the researcher, the teacher involved and the principal and resubmitted to the Victoria University ethics committee for final approval. Permission to have conversations with students while 
they are doing the activity will be sort by the researcher, to elicit why they are adding certain attributes to their poster.

Off site, I will be reflecting on my experiences at the school via my research journal. Here I will be making notes of the climate of the school, how my interactions have gone and what I could be doing differently or better as a researcher. I am happy to have these field notes available for you to see.

\section{Confidentiality and access to information}

The information provided is strictly confidential. Your school and you, the participant, will not be identifiable. Information collected will be kept in a locked filing cabinet at the office of Angela Millar, Victoria University of Wellington.

Feedback will be provided through ongoing discussions as well as summary reports. During ongoing discussions as well as after been given summary reports, participants will have the chance to review their answers. They will be given the opportunity to change or delete their comments if they wish.

\section{The participation for the staff would involve...}

- Taking part in up to two focus group meetings.

- The opportunity to elect your class to take part in an in-class activity.

- Being available for further interviews or discussions should that be required.

- Be available to read a summary report and offer feedback as required.

- Agreeing that the information given can be used by the researcher, Angela Millar for this study.

\section{Publication of Results}

The information gained in this research will be used for publication purposes including academic or professional journals, conference papers and appropriate articles and for the deposit of the thesis at the Victoria University of Wellington library.

Your consent for this study is highly valued.

Thank you

Angela Millar 
TE WHARE WĀNANGA O TE ŪPOKO O TE IKA A MĀUI

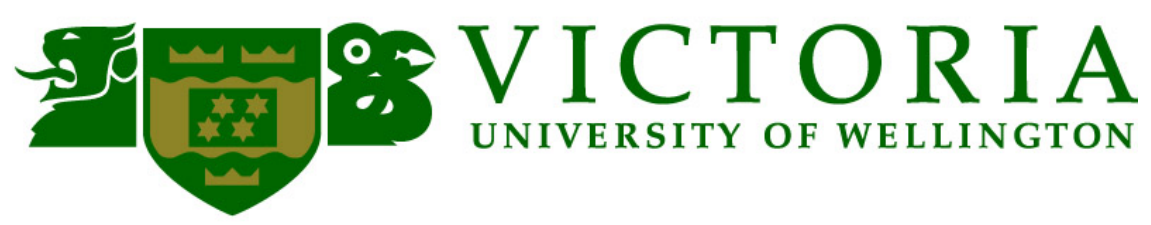

$\underline{\text { Research Project, Community information sheet }}$

"An investigation into leadership practice in low socio-economic New

Zealand primary schools: How principal leaders engage for educational success."

\section{Researcher}

I am Angela Millar, a PhD student at the Faculty of Education, Victoria University of Wellington, New Zealand. As part of my doctoral study, I am conducting research to examine the role of principal leadership practices in promoting educational outcomes in low socio- economic primary school settings. This research has ethical approval from the Victoria University College of Education Ethics Committee.

This sheet gives you information about this study. If you have any questions or would like to receive further information about the project, please contact me at xxxx. You can also contact my supervisor Kate Thornton at xxxx, and Liz Jones at xxx.

The research has been approved by the Faculty of Education Human Ethics Subcommittee under delegated authority from the Victoria University Human Ethics Committee. If you have any ethical questions about this research please contact Dr Allison Kirkman, Chair of the Human Ethics Committee, Victoria University of Wellington on $\mathrm{xxx}$.

\section{The purpose of my $\mathrm{PhD}$ research}

This research aims to contribute to the knowledge base of how principal leadership practice impacts on educational success within the social complexities of a low socio economic schooling community.

\section{Research questions}

"In what ways do principals practice leadership in low socio economic primary school contexts to enhance educational success?"

- What do the stakeholders in the LSE context perceive as educational success?

- What leadership practice patterns are in evidence in the daily life of a principal?

- What ways are principal practices influenced by or influencing of their context?

- What beliefs and values underpin principal's engagement in their context?

\section{Research process}

My study will be conducted in three primary schools across New Zealand. The school and your principal have been chosen because together they meet the criteria for participating in research aiming to develop an understanding of the phenomenon of 
effective principal leadership in a low socio economic schooling context. To achieve an in-depth understanding, I plan to include several data gathering methods in the research process.

By observing and shadowing the principal in action, interviewing the principal and school community and gathering documentation over a term, I will be able to address the research questions as well as describe the phenomenon of effective principal leadership from within your unique school context. I may return to the school once or twice the following term for any necessary follow up.

\section{Research participants}

I am asking for the principal and volunteers from the school's community to take part in this research. This is an opportunity to ensure all those interested, are given the opportunity to represent their understandings of how principal leadership practices support educational success.

\section{The group interview \& the researcher journal}

After gaining permission from the BOT of $\mathrm{x}$ School and the principal to undertake the research, I will conduct a community focus group session, which I would value your input on. This activity will be done during my data collection from $\mathrm{xxx}$ to $\mathrm{xxx}$ in school. Group interviews lasting a maximum of an hour and a half will be held with volunteers from the community. These will be audio recorded. This group will discuss such things as their concepts of educational success as well as the principal's role in creating this.

Off site, I will be reflecting on my experiences at the school via my research journal. Here I will be making notes of the climate of the school, how my interactions have gone and what I could be doing differently or better as a researcher. I am happy to have these field notes available for you to see.

\section{Confidentiality and access to information}

The information provided is strictly confidential and you will not be identifiable. Information collected will be kept in a locked filing cabinet at the office of Angela Millar, Victoria University of Wellington.

Feedback will be provided through ongoing discussions as well as summary reports. During ongoing discussions as well as after been given summary reports, participants will have the chance to review their answers. They will be given the opportunity to change or delete their comments if they wish.

\section{Participation}

Participants may withdraw from the research project for any reason at any stage prior to the data analysis and any data provided would then be destroyed.

\section{The participation of community members who volunteer would involve:}

- taking part in up to two focus group meetings.

- being available for further interviews or discussions should that be required. 
- be available to read a summary report and offer feedback as required.

- agreeing that the information given can be used by the researcher, Angela Millar for this study.

\section{Publication of results}

The information gained in this research will be used for publication purposes including academic or professional journals, conference papers and appropriate articles and for the deposit of the thesis at the Victoria University of Wellington library.

Your consent for this study is highly valued. Thank you.

Angela Millar 
TE WHARE WĀNANGA O TE ŪPOKO O TE IKA A MĀUI

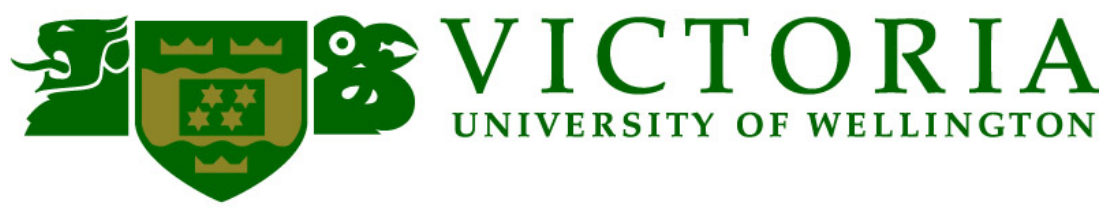

\section{$\underline{\text { Research Project - Student information sheet }}$}

"An investigation into leadership practice in low socio-economic New Zealand primary schools: How principal leaders engage for educational success."

\section{Researcher}

I am Angela Millar, a PhD student at the Faculty of Education, Victoria University of Wellington, New Zealand. As part of my doctoral study, I am conducting research to examine the role of principal leadership practices in promoting educational outcomes in low socio- economic primary school settings. This research has ethical approval from the Victoria University College of Education Ethics Committee.

This sheet gives you information about this study. If you have any questions or would like to receive further information about the project, please contact me at xxxx. You can also contact my supervisor Kate Thornton at xxxx, and Liz Jones at xxx.

The research has been approved by the Faculty of Education Human Ethics Subcommittee under delegated authority from the Victoria University Human Ethics Committee. If you have any ethical questions about this research please contact Dr Allison Kirkman, Chair of the Human Ethics Committee, Victoria University of Wellington on $\mathrm{xxx}$.

\section{The purpose of my PhD research}

This research aims to contribute to the knowledge base of how principal leadership practices impact on educational success within the social complexities of your schooling community.

\section{Research questions}

"In what ways do principal leaders engage in low socio economic primary school contexts to enhance educational success?"

- What do the stakeholders in the LSE context perceive as educational success?

- What leadership practices are in evidence in the daily life of a principal?

- In what ways are principal practices adaptive to their context?

- What beliefs and values underpin principal's engagement in their context?

\section{Research process}

My study will be conducted in three primary schools across New Zealand. The 
school and your principal have been chosen because together they meet the criteria for participating in research aiming to develop an understanding of the phenomenon of effective principal leadership in a low socio economic schooling context. To achieve an in-depth understanding, I plan to include several data gathering methods in the research process.

By observing and shadowing the principal in action, interviewing the principal and school community and gathering documentation over a term, I will be able to address the research questions as well as describe the phenomenon of effective principal leadership from within your unique school context. I may return to the school once or twice the following term for any necessary follow up.

\section{Research participants}

I am asking for the principal and volunteers from the school's community to take part in this research. This is an opportunity to ensure all those interested are given the opportunity to represent their understandings of how principal leadership practices support educational success.

\section{The group interview $\&$ the researcher journal}

After gaining permission on the $\mathrm{X}$, from the BOT of X School and the principal to undertake the research, I will conduct an in-class activity, which your son/daughter's teacher has indicated an interest in involving his/her class in. I would value your son/daughter's input on. This activity will be done during my data collection from $\mathrm{xxx}$ to $\mathrm{xxx}$. The in-class activity will last a maximum of an hour and a half will be run by the classroom teacher. The session will be audio recorded. The class will discuss such things as their concepts of educational success as well their perceptions of the principal's practice.

Off site, I will be reflecting on my experiences at the school via my research journal. Here I will be making notes of the climate of the school, how my interactions have gone and what I could be doing differently or better as a researcher. I am happy to have these field notes available for you to see.

\section{Confidentiality and access to information}

The information provided is strictly confidential and your son/daughter will not be identifiable. Information collected will be kept in a locked filing cabinet at the office of Angela Millar, Victoria University of Wellington. During and at the completion of the in-class activity I will check with students that I have recorded their ideas accurately. If there is any uncertainty I will ask for elaboration or further explanation. They will be given the opportunity to change or delete ideas and concepts as they wish.

\section{Participation}

Students may withdraw from the research project for any reason at any stage prior to the data analysis and any data provided would then be destroyed. 


\section{The participation of students who volunteer would involve}

- Taking part in the in-class activity.

- Agree to the researcher being present at the activity.

- Being available for further interviews or discussions should that be required.

- Agreeing that the information given can be used by the researcher, Angela Millar for this study.

\section{Publication of results}

The information gained in this research will be used for publication purposes including academic or professional journals, conference papers and appropriate articles and for the deposit of the thesis at the Victoria University of Wellington library.

Your consent for this study is highly valued. Thank you.

Angela Millar 


\section{Appendix M:}

\section{Consent forms - staff, community and students}

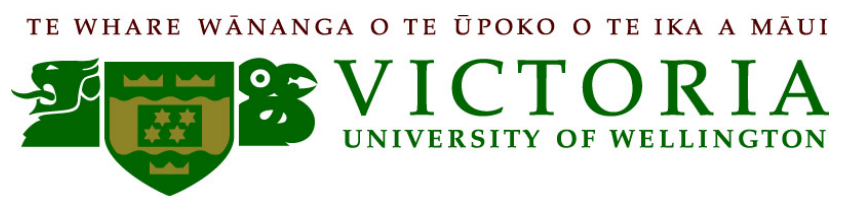

\section{$\underline{\text { Research Project- Staff consent form }}$}

"An investigation into leadership practice in low socio-economic New Zealand primary schools: How principal leaders engage for educational success."

\section{Please read the following:}

I agree to take part in up to two focus group meetings.

I I will be available for further interviews or discussions should that be required.

$>$ I understand that my participation is entirely voluntary.

$>$ I understand that the research is confidential.

$>$ I understand that the analysis and aggregation of the data obtained may be used for a thesis, for conference papers and/or publication by the researcher Angela Millar.

$>$ I understand that the data collected will only be used for the research project as described in the information sheet.

$>$ I understand that I can withdraw from the research project for any reason at any stage prior to the data analysis and that any data provided would then be destroyed.

$>$ I understand that I will have a chance to check the accuracy of any interviews and observations.

$>$ I understand that transcripts and tapes and raw data will be destroyed five years after the conclusion of the project.

\section{Please indicate your preference for involvement in the research (tick as appropriate):}

I have had the project explained and have had the chance to ask any questions. I do not give consent to take part in the research.

- I would like further questions to be answered about this project.

- I have had the project explained to me and have had the chance to ask any questions. I agree to taking part in the above research. 


\section{Community Request for Research Summary}

I wish to be given a summary of the research findings.

○ Yes

$\circ \quad$ No

Name:

School:

This research has ethical approval from the Victoria University Faculty of Education 


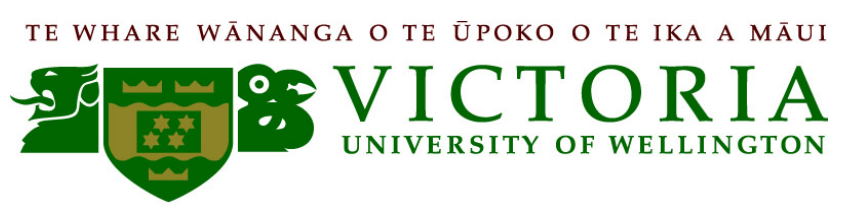

\section{$\underline{\text { Research Project- Community consent form }}$}

"An investigation into leadership practice in low socio-economic New Zealand primary schools: How principal leaders engage for educational success."

\section{Please read the following:}

$>$ I agree to take part in up to two focus group meetings.

I I will be available for further interviews or discussions should that be required.

$>$ I understand that my participation is entirely voluntary.

$>$ I understand that the research is confidential.

$>$ I understand that the analysis and aggregation of the data obtained may be used for a thesis, for conference papers and/or publication by the researcher Angela Millar.

$>$ I understand that the data collected will only be used for the research project as described in the information sheet.

$>$ I understand that I can withdraw from the research project for any reason at any stage prior to the data analysis and that any data provided would then be destroyed.

$>$ I understand that I will have a chance to check the accuracy of any interviews and observations.

I understand that transcripts and tapes and raw data will be destroyed five years after the conclusion of the project.

Please indicate your preference for involvement in the research (tick as appropriate):

I have had the project explained and have had the chance to ask any questions. I do not give consent to take part in the research.

- I would like further questions to be answered about this project.

- I have had the project explained to me and have had the chance to ask any questions. I agree to taking part in the above research.

Name Name of school

Signature Date 


\section{Community request for research summary}

I wish to be given a summary of the research findings.
○ Yes
- No

Name:

School:

This research has ethical approval from the Victoria University Faculty of Education 


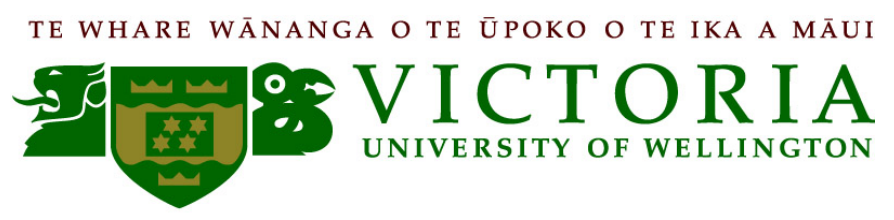

\section{$\underline{\text { Research Project- student consent form }}$}

"An investigation into leadership practice in low socio-economic New Zealand primary schools: How principal leaders engage for educational success."

\section{Please read the following:}

$>$ I agree to my son/daughter taking part in an in-class activity.

I agree to the researcher Angela Millar being present during this in-class activity.

$>$ I agree that the researcher Angela Millar, may ask my son/daughter questions pertaining to their work during the session.

$>$ I understand that my son/daughter's participation is entirely voluntary.

$>$ I understand that the research is confidential.

$>$ I understand that the analysis and aggregation of the data obtained may be used for a thesis, for conference papers and/or publication by the researcher Angela Millar.

$>$ I understand that the data collected will only be used for the research project as described in the information sheet.

$>$ I understand that I/my child can withdraw from the research project for any reason at any stage prior to the data analysis and that any data provided would then be destroyed.

$>$ I understand that my son/daughter will have a chance to check the accuracy of the observation/s.

$>$ I understand that transcripts and tapes and raw data will be destroyed five years after the conclusion of the project.

Please indicate your preference for involvement in the research (tick as appropriate):

- I have had the project explained and have had the chance to ask any questions. I do not give consent for my son/daughter to take part in the research.

- I would like further questions to be answered about this project.

- I have had the project explained to me and have had the chance to ask any questions. I agree to my son/ daughter taking part in the above research.

Name School

Signature Date 


\section{Request for Research Summary}

I wish to be given a summary of the research findings.

$$
\begin{array}{cc}
\circ & \text { Yes } \\
\circ & \text { No }
\end{array}
$$

Name:

School:

This research has ethical approval from the Victoria University College of Education Ethics Committee. 


\section{Appendix N:}

\section{Formal observations- information and consent forms}

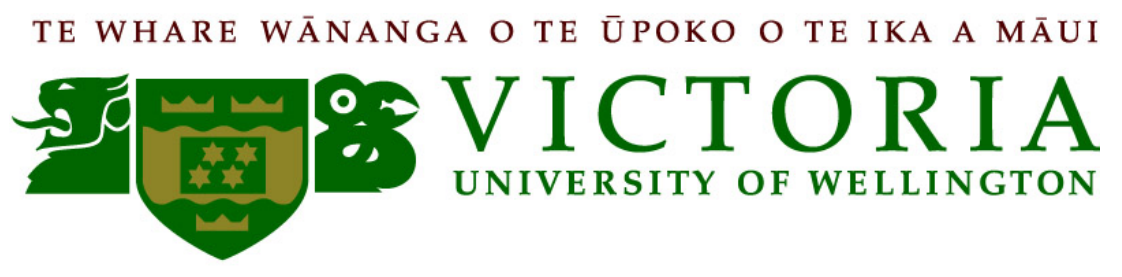

$\underline{\text { Research project, formal observation information sheet }}$

"An investigation into leadership practice in low socio-economic New Zealand primary schools: How principal leaders engage for educational success."

\section{Researcher}

I am Angela Millar, a PhD student at the Faculty of Education, Victoria University of Wellington, New Zealand. As part of my doctoral study, I am conducting research to examine the role of principal leadership practices in promoting educational outcomes in low socio- economic primary school settings. This research has ethical approval from the Victoria University College of Education Ethics Committee.

This sheet gives you information about this study. If you have any questions or would like to receive further information about the project, please contact me at xxxx. You can also contact my supervisor Kate Thornton at xxxx, and Liz Jones at xxx.

The research has been approved by the Faculty of Education Human Ethics Subcommittee under delegated authority from the Victoria University Human Ethics Committee. If you have any ethical questions about this research please contact Dr Allison Kirkman, Chair of the Human Ethics Committee, Victoria University of Wellington on $\mathrm{xxx}$.

\section{The purpose of my $\mathrm{PhD}$ research}

This research aims to contribute to the knowledge base of how principal leadership practices impact on educational success within the social complexities of a low socio economic schooling community.

\section{Research question/s}

"In what ways do principal leaders engage in low socio economic primary school contexts to enhance educational success?"

- What do the stakeholders in the LSE context perceive as educational success?

- What leadership practices are in evidence in the daily life of a principal?

- In what ways are principal practices adaptive to their context?

- What beliefs and values underpin principal's engagement in their context? 


\section{Research process}

My study will be conducted in three primary schools across New Zealand. The school and your principal have been chosen because together they meet the criteria for participating in research aiming to develop an understanding of the phenomenon of effective principal leadership in a low socio economic schooling context. To achieve an in-depth understanding, I plan to include several data gathering methods in the research process.

By observing and shadowing the principal in action, interviewing the principal and school community and gathering documentation over a term, I will be able to address the research questions as well as describe the phenomenon of effective principal leadership from within your unique school context. I may return to the school once or twice the following term for any necessary follow up.

\section{Research participants}

I am asking for the principal and volunteers from the school's community to take part in this research. This is an opportunity to ensure all those interested, are given the opportunity to represent their understandings of how principal leadership practices support educational success.

\section{The formal observations \& the researcher diary}

After gaining permission from the BOT of X School and the principal X to undertake the research, I will conduct a formal observation which I understand you will be present at. This observation will be done during my data collection from $\mathrm{X}$ to $\mathrm{X}$ in school as part of the usual term's programme of events. The observation will last the entire duration of the activity. These will be audio and/or video recorded.

During and at the completion of the formal observation I will summarize my findings. If there is any uncertainty I will ask for elaboration or further explanation. You will be given the opportunity to change or delete comments as you wish. Feedback will also be provided through a summary report.

Off site, I will be reflecting on my experiences at the school via my research journal. Here I will be making notes of the climate of the school, how my interactions have gone and what I could be doing differently or better as a researcher. I am happy to have these field notes available for you to see.

\section{Confidentiality and access to information}

The information provided is strictly confidential. As a participant, you will not be identifiable. Information collected will be kept in a locked filing cabinet at the office of Angela Millar, Victoria University of Wellington.

\section{Participation}

Participants may withdraw from the research project for any reason at any stage prior to the data analysis and any data provided would then be destroyed. 
Participation for you would involve...

- Taking part in the activity the formal observation is based around.

- Being available for further discussion should that be required.

- Be available to read a summary report and offer feedback as required.

- Agreeing that the information given can be used by the researcher, Angela Millar for this study.

\section{Publication of results}

The information gained in this research will be used for publication purposes including academic or professional journals, conference papers and appropriate articles and for the deposit of the thesis at the Victoria University of Wellington library.

Your consent for this study is highly valued. Thank you.

Angela Millar 
CONSENT FORM - FORMAL OBSERVATION

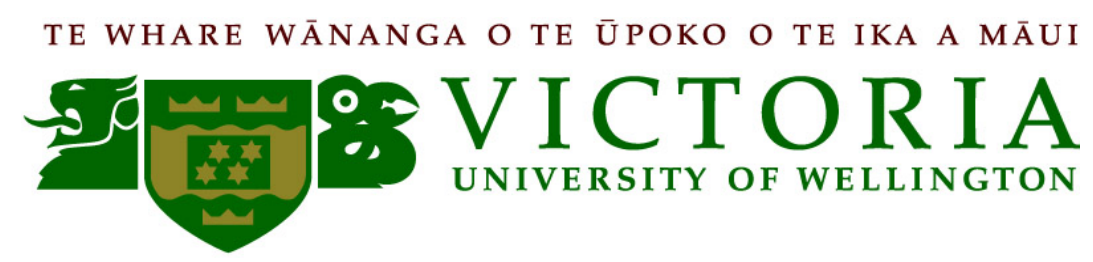

\section{$\underline{\text { Research Project- Formal Observation Consent Form }}$}

"An investigation into leadership practice in low socio-economic New Zealand primary schools: How principal leaders engage for educational success."

\section{Please read the following:}

I agree to be a part of up to two informal observations where the principal is actively involved.

$>$ I will be available for further interviews or discussions should that be required.

$>$ I understand that the activity will be videoed OR audio recorded.

$>$ I understand that my participation is entirely voluntary.

$>$ I understand that the research is confidential.

$>$ I understand that the analysis and aggregation of the data obtained may be used for a thesis, for conference papers and/or publication by the researcher Angela Millar.

$>$ I understand that the data collected will only be used for the research project as described in the information sheet.

$>$ I understand that I can withdraw from the research project for any reason at any stage prior to the data analysis and that any data provided would then be destroyed.

$>$ I understand that I will have a chance to check the accuracy of the observation/s.

$>$ I understand that transcripts and tapes and raw data will be destroyed five years after the conclusion of the project. 
Please indicate your preference for involvement in the research (tick as appropriate):

I have had the project explained and have had the chance to ask any questions. I do not give consent to take part in the research.

- I would like further questions to be answered about this project.

- I have had the project explained to me and have had the chance to ask any questions. I agree to taking part in the above research.

Name School

Signature Date

\section{Request for Research Summary}

I wish to be given a summary of the research findings.

○ Yes

○ No

Name:

School:

This research has ethical approval from the Victoria University Faculty of Education Ethics Committee. 